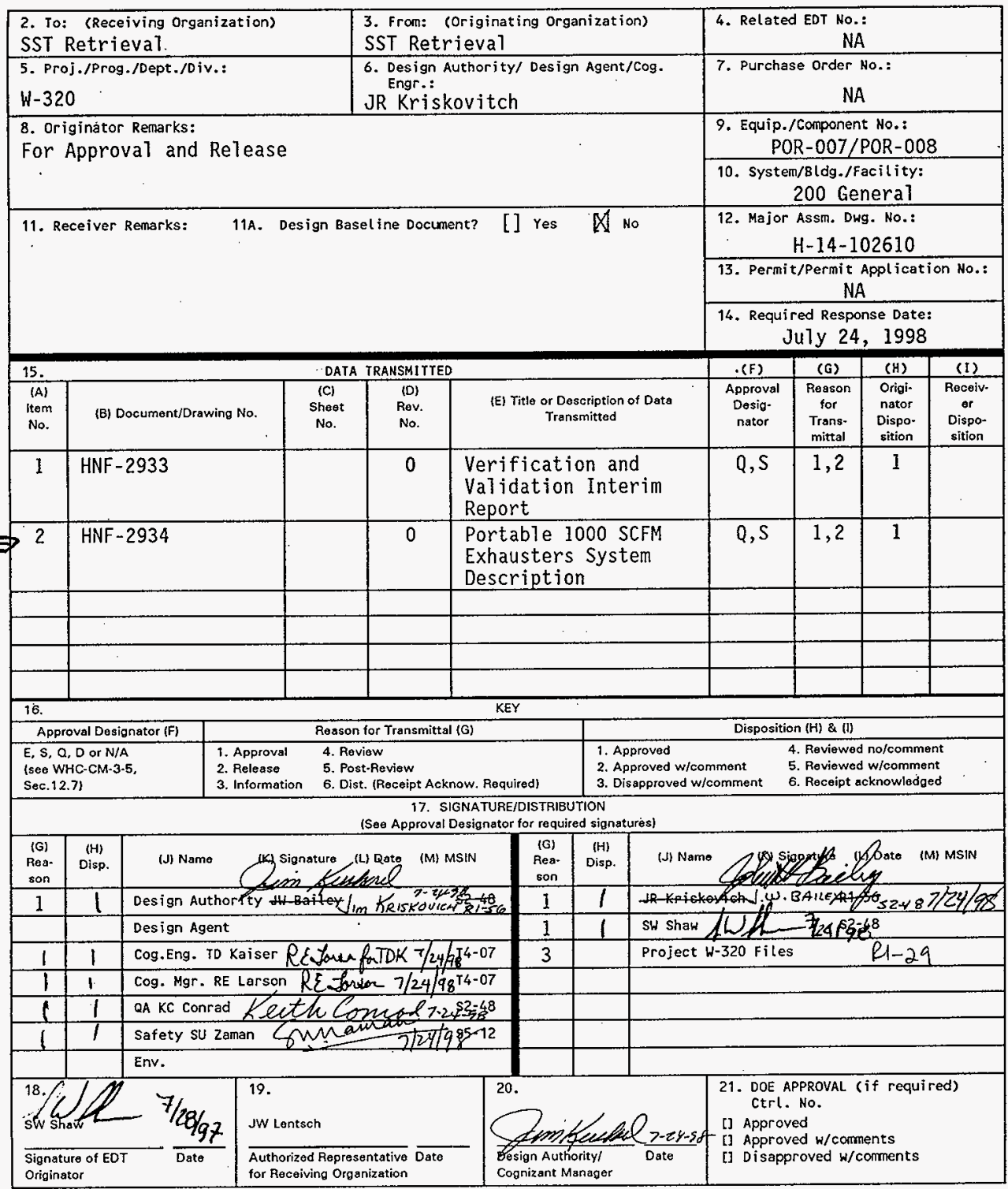




\section{System Design Description for Portable 1,000 CFM Exhauster Skids POR-007/Skid E and POR- 008/Skid F}

OD Nelson

NHC, Richland, WA 99352

U.S. Department of Energy Contract DE-AC06-96RL13200

EDT/ECN: 622196

Org Code: 8C452

UC: 510

B\&R Code: EW3130010

Charge Code: D2M65

Total Pages: 151

Key Words: POR-007, POR-008, System Design Description, W-320, Control Mode, 1,000 CFM, Exhauster Skid, Programmable Logic Controller (PLC), STuicing

Abstract: Provides the system design description for the 1,000 CFM Portable Exhauster skids. The different control modes are described: flow, fan inlet pressure, and high vacuum.

IRADEMARK DISCLAIMER. Reference here in to any specific comercial product, process, or service by trade name, tradenark, manufacturer, or otherwise, does not necessarily constitute or imply its endorsement, recomendation, or favoring by the United States Government or any agency thereof or its contractors or subcontractors.

Printed in the United States of America. To obtain copies of this document, contact: Document Control Services, P.O. Box 950, Maïlstop H6-08, Richland WA 99352, Phone (509) 372-2420;

Fax (509) 376-4989.

Aniskradex

Reloase Approval
7125198

Date

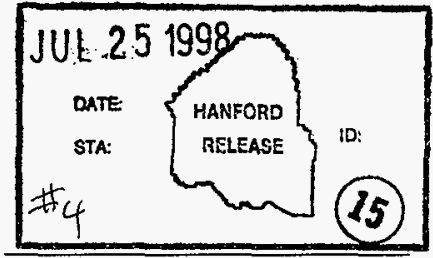

Release Stamp 


\section{SYSTEM DESIGN DESCRIPTION}

FOR

PORTABLE 1,000 CFM EXHAUSTER SKIDS

POR-007-SKIDE

AND

POR-008-SKID F

Document Identifier (DI): HNF-2934

Revision 0

July 23, 1998

Prepared by:

Fluor Daniel Northwest (FDNW)

Control Systems Engineering CCAD Team

1200 Jadwin Avenue

Richland, WA

Authored by:

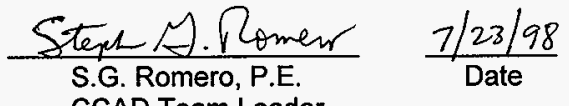
CCAD Team Leader 
Title: Portable 1,000 CFM Exhausters

System Design Description (SDD)
DI: HNF-2934

Rev. 0

Table of Revisions

\begin{tabular}{|c|c|l|l|}
\hline Rev. & $\begin{array}{c}\text { Date of } \\
\text { Issue }\end{array}$ & \multicolumn{1}{|c|}{ Description } & Remarks \\
\hline 0 & $7 / 23 / 98$ & Issued for approval & \\
\hline & & & \\
\hline & & & \\
\hline & & & \\
\hline
\end{tabular}


Title: Portable 1,000 CFM Exhausters System Design Description (SDD)
DI: HNF-2934

Rev. 0

\section{TABLE OF CONTENTS}

INTRODUCTION

1.1 PURPOSE

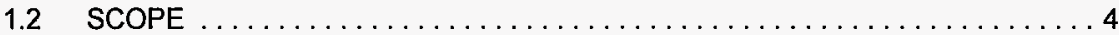

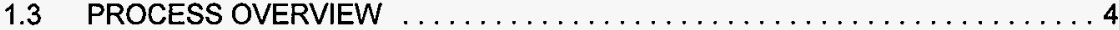

1.3.1 TANK DOME SPACE VENTILATION $\ldots \ldots \ldots \ldots \ldots \ldots \ldots \ldots$

1.3.2 TANK ANNULUS VENTILATION $\ldots \ldots \ldots \ldots \ldots \ldots \ldots \ldots \ldots \ldots \ldots$

1.4 DEFINITIONS ......................... 5

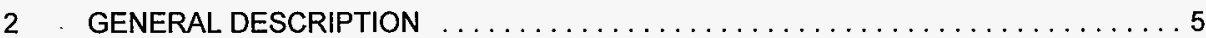

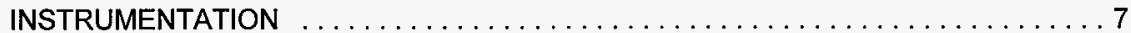

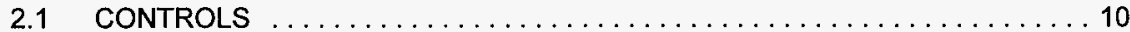

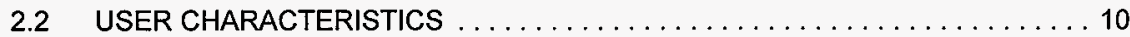

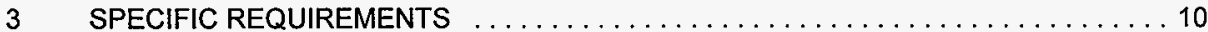

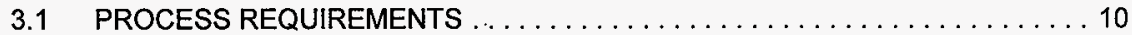

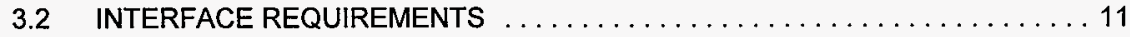

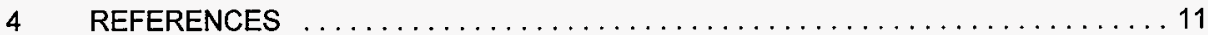

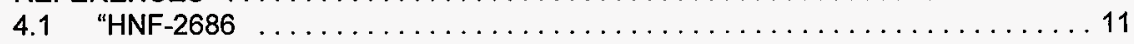

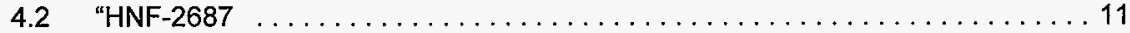

5 DESCRIPTION OF PLC CONTROL AND PROGRAM $\ldots \ldots \ldots \ldots \ldots \ldots \ldots \ldots 11$

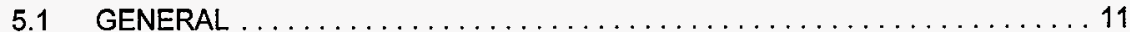

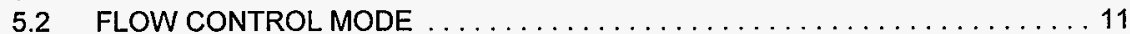

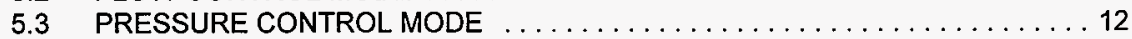

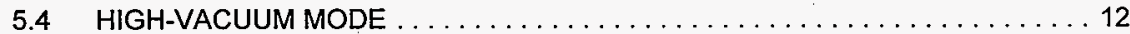

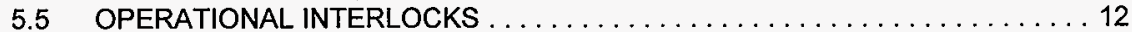


Title: Portable 1,000 CFM Exhausters System Design Description (SDD)
DI: HNF-2934

Rev. 0

INTRODUCTION

\subsection{PURPOSE}

The primary purpose of the two 1,000 SCFM Exhauster Skids, POR-007-SKID E and POR-008-SKID $F$ is to provide backup to the waste tank primary ventilation systems for tanks $241-C-106$ and 241AY-102, and the AY-102 annulus in the event of a failure during the sluicing of tank 241-C-106 and subsequent transfer of sluiced waste to 241-AY-102. This redundancy is required since both of the tank ventilation systems have been declared as Safety Class systems.

\subsection{SCOPE}

One Exhauster Skid will provide the tank ventilation backup function for the first year of sluicing, at which time it will be returned to the Salt Well pumping group provided it has not actually been put into service.

\subsection{PROCESS OVERVIEW}

The exhauster skids $E$ and $F$ are identical and so the following discussions will speak of only one skid. The exhauster skid may be used in one of two modes: tank dome space ventilation or tank annulus ventilation. The difference lies primarily in the process variable used to control speed of the fan. For tank dome space ventilation (Flow Control Mode), the exhauster skid stack flow is used as the process variable. For tank annulus ventilation (Pressure Control), the exhauster skid fan inlet pressure (vacuum) is used. The instrumentation and controls for the skid operations will be discussed in more detail in later sections of this document.

\subsubsection{TANK DOME SPACE VENTILATION}

For tank C-106 dome space ventilation, the exhauster skid suction is connected to tank riser 19B and pulls air out of the tank dome space through the skid-mounted equipment in the following order:

- Manual flow control valve (VTP-V-135)

- 1st transition piece

- Demister (VTP-HX-001)

- $\quad$ Pre-filter section (VTP-FLT-001)

- Test section 1

- $\quad$ HEPA section 1 (VTP-FLT-002)

- Test section 2

- HEPA section 2 (VTP-FLT-003)

- 2nd transition piece

- $\quad$ Flow control valve (VTP-V-136)

- Expansion joint

- $\quad$ Exhaust fan (VTP-EF-001)

- $\quad$ Skid stack

See drawing $\mathrm{H}-14-102610$, sheet 1 , for the skid P\&ID. 
Title: Portable 1,000 CFM Exhausters System Design Description (SDD)
DI: HNF-2934

Rev. 0

A glycol heater (VTP-HTR-001) is used to heat the tank air after it travels through the demister. The glycol recirc pump (VTP-P-001) is used to recirculate the glycol through the heater and the heater coil in the demister.

Any condensate from any of the skid sections, including the exhaust fan, drains into the seal pot (VTP-SP-001). A seal pot pump (VTP-P-004) is used to pump the condensate out of the seal pot.

\subsubsection{TANK ANNULUS VENTILATION}

For tank annulus ventilation, the exhauster skid suction is connected to a suitable tank annulus riser and pulls air out of the tank annulus and through the skid-mounted equipment described in the previous section.

\subsection{DEFINITIONS}

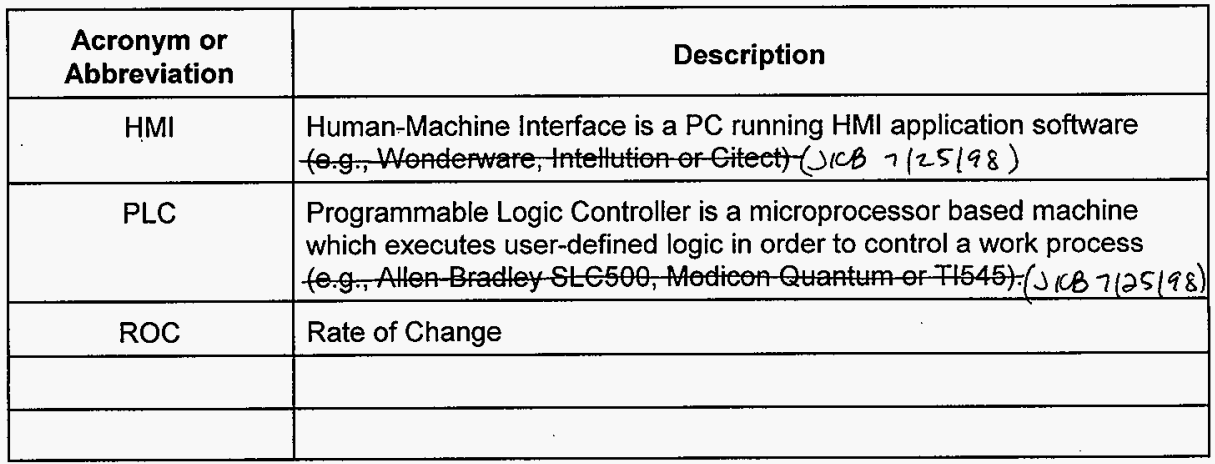

\section{GENERAL DESCRIPTION}

The exhauster skid, in addition to the mechanical equipment described above, is provided with several instrumentation and control devices. There are transmitters used to measure several process variables, panel mounted indicators to display the process variables, panel mounted control switches, message view displays for alarm indication and a heat-trace cabinet which houses alarm trips for plenum pressures and flows.

The process instrumentation and control devices provide input and output (I/O) signals to the PLC mounted inside the control panel. The PLC is programmed to use either exhaust flow or fan inlet vacuum as its process variable input to be controlled, for use with primary tank or annulus ventilation, respectively. It is programmed to provide safety interlocks, as well as, alarm messages which are shown on the message view displays. An unacknowledged alarm on the PLC will cause the alarm beacon mounted high on the skid to rotate and flash. Acknowledgment of alarms is done at the message view displays. 
Title: Portable 1,000 CFM Exhausters System Design Description (SDD)

DI: HNF-2934

Rev. 0

Electrical equipment includes a main skid disconnect switch and disconnect switches for the exhaust fan and glycol heater. There is a variable speed drive mounted inside the control panel for controlling the exhaust fan speed. A mini-power center provides for instrument and control power. 
Title: Portable 1,000 CFM Exhausters

System Design Description (SDD)
DI: HNF-2934

Rev. 0

\section{INSTRUMENTATION}

The following table describes the skid instrumentation and lists each instrument by tagname along with any alarms associated with the process variable.

TABLE 2.1 - SKID INSTRUMENTATION ${ }^{1}$

\begin{tabular}{|c|c|c|c|c|c|c|}
\hline Item & Tagname & Description & $\begin{array}{l}\text { Manufacturer \& Model } \\
\text { No. }\end{array}$ & $\begin{array}{c}\text { Range } \\
\text { Or Setpoint }\end{array}$ & Alarms & $\begin{array}{l}\text { Alarm } \\
\text { Setpoints }\end{array}$ \\
\hline 1 & VTP-PDT-170A & $\begin{array}{l}\text { Plenum Pressure Transmitter } \\
\text { (Mounted on Exhaust Fan Suction) }\end{array}$ & Yokogawa EJA-110A & $\begin{array}{c}0 \text { to }-20^{\prime \prime} W C \\
\left(0^{\prime \prime}=4 \mathrm{~mA}\right) \\
\left(-20^{\prime \prime}=20 \mathrm{~mA}\right)\end{array}$ & $\begin{array}{c}\text { PAH-170A (V) } \\
\text { PAH-170A (HV) } \\
\text { PAL-170A }\end{array}$ & $\begin{array}{l}-12.75^{\prime \prime} \text { W.C. } / \\
-19.5^{\prime \prime} \text { W.C. } \\
-6.0^{\prime \prime} \text { W.C. }\end{array}$ \\
\hline 2 & VTP-PDT-170B & $\begin{array}{l}\text { Plenum Pressure Transmitter } \\
\text { (Mounted on Skid Inlet) }\end{array}$ & Yokogawa EJA-110 & $\begin{array}{c}-5 " \text { to }+5^{\prime \prime} W C \\
\left(-5^{\prime \prime}=4 \mathrm{~mA}\right) \\
\left(+5^{\prime \prime}=20 \mathrm{~mA}\right)\end{array}$ & $\begin{array}{l}\text { PAH-170B } \\
\text { PAL-170B }\end{array}$ & $\begin{array}{l}+5.0^{\prime \prime} \text { W.C. } \\
-3.5^{\prime \prime} \text { W.C. }\end{array}$ \\
\hline 3 & VTP-PDT-177 & $\begin{array}{l}\text { Heater/Demister Differential Pressure } \\
\text { Transmitter }\end{array}$ & Yokogawa EJA-120 & $\begin{array}{c}0-2^{\prime \prime} W C \\
\left(0^{\prime \prime}=4 \mathrm{~mA}\right) \\
\left(2^{\prime \prime}=20 \mathrm{~mA}\right)\end{array}$ & $\mathrm{N} / \mathrm{A}$ & $\mathrm{N} / \mathrm{A}$ \\
\hline 4 & VTP-TE-176 & Inlet Temperature Thermocouple & $\begin{array}{l}\text { Omega (Model No. not } \\
\text { visible) }\end{array}$ & Type J & N/A & N/A \\
\hline 5 & VTP-PDT-178 & $\begin{array}{l}\text { Prefilter Differential Pressure } \\
\text { Transmitter }\end{array}$ & Yokogawa EJA-120 & $\begin{array}{c}0-2^{\prime \prime} W C \\
\left(0^{\prime \prime}=4 \mathrm{~mA}\right) \\
\left(2^{n}=20 \mathrm{~mA}\right)\end{array}$ & PDAH -178 & 1" W.C. \\
\hline 6 & VTP-TE-179 & $\begin{array}{l}\text { HEPA Filter \#1 Inlet Temperature } \\
\text { Thermocouple }\end{array}$ & $\begin{array}{l}\text { Omega (Model No. not } \\
\text { visible) }\end{array}$ & Type J & $\begin{array}{l}\text { TAH-179 } \\
\text { TAL- } 179 \\
\end{array}$ & $\begin{array}{l}190^{\circ} \mathrm{F} \\
40^{\circ} \mathrm{F}\end{array}$ \\
\hline 7 & VTP-PDT-180 & $\begin{array}{l}\text { HEPA Filter \#1 Differential Pressure } \\
\text { Transmitter }\end{array}$ & Yokogawa EJA-110 & $\begin{array}{c}0-10^{\prime \prime} \text { WC } \\
\left(0^{\prime \prime}=4 \mathrm{~mA}\right) \\
\left(10^{\prime \prime}=20 \mathrm{~mA}\right)\end{array}$ & $\begin{array}{c}\text { PDAHH-180 } \\
\text { PDAH-180 } \\
\text { PDAL-180 } \\
\text { ROC-180 }\end{array}$ & $\begin{array}{l}5.4^{\prime \prime} \text { W.C. } \\
4.5^{\prime \prime} \text { W.C. } \\
0.1^{\prime \prime} \text { W.C. } \\
0.5^{\prime \prime} \text { W.C. }\end{array}$ \\
\hline
\end{tabular}

${ }^{1}$ Alarm tags from P\&ID H-14-102610. Alarm Setpoints from System Variables drawing H-14-102608. Descriptions match nameplates and steel tags on skid. 
Title: Portable 1,000 CFM Exhausters

System Design Description (SDD)
DI: HNF-2934

Rev. 0

\begin{tabular}{|c|c|c|c|c|c|c|}
\hline Item & Tagname & Description & $\begin{array}{l}\text { Manufacturer \& Model } \\
\text { No. }\end{array}$ & $\begin{array}{c}\text { Range } \\
\text { Or Setpoint }\end{array}$ & Alarms & $\begin{array}{l}\text { Alarm } \\
\text { Setpoints }\end{array}$ \\
\hline 8 & VTP-PDT-181 & $\begin{array}{l}\text { Filter Train Differential Pressure } \\
\text { Transmitter }\end{array}$ & Yokogawa EJA-110 & $\begin{array}{c}0-6^{\prime \prime} \text { WC } \\
\left(0^{\prime \prime}=4 \mathrm{~mA}\right) \\
\left(6^{\prime \prime}=20 \mathrm{~mA}\right)\end{array}$ & $\begin{array}{l}\text { PDAHH-181 } \\
\text { PDAL-181 }\end{array}$ & $\begin{array}{l}\text { 5.4" W.C. } \\
0.1^{\prime \prime} \text { W.C. }\end{array}$ \\
\hline 9 & VTP-PDT-182 & $\begin{array}{l}\text { HEPA Filter \#2 Differential Pressure } \\
\text { Transmitter }\end{array}$ & Yokogawa EJA-110 & $\begin{array}{c}0-6^{\prime \prime} \mathrm{WC} \\
\left(0^{\prime \prime}=4 \mathrm{~mA}\right) \\
\left(6^{\prime \prime}=20 \mathrm{~mA}\right)\end{array}$ & $\begin{array}{l}\text { PDAHH-182 } \\
\text { PDAH-182 } \\
\text { PDAL-182 } \\
\text { ROC-182 }\end{array}$ & $\begin{array}{l}\text { 3.7" W.C. } \\
3.2^{\prime \prime} \text { W.C. } \\
0.1^{\prime \prime} \text { W.C. } \\
0.5^{\prime \prime} \text { W.C. }\end{array}$ \\
\hline 10 & VTP-TE-183 & Stack Temperature Thermocouple & $\begin{array}{l}\text { Omega (Model No. not } \\
\text { visible) }\end{array}$ & Type J & N/A & N/A \\
\hline 11 & VTP-FT-184 & $\begin{array}{l}\text { Stack Flow Transmitter } \\
\text { (Calculated from Differential } \\
\text { Pressure) }\end{array}$ & Yokogawa EJA-120 & $\begin{array}{l}0-1194 \text { SCFM } \\
(0-4 " \text { WC) }\end{array}$ & $\begin{array}{c}\text { FAH-184 } \\
\text { FAH-184 (V/HV) } \\
\text { FAL-184 } \\
\text { FAL-184 (V/HV) }\end{array}$ & $\begin{array}{c}1100 \\
\text { SCFM } \\
1000 \text { SCFM } \\
470 \text { SCFM } \\
675 \text { SCFM }\end{array}$ \\
\hline 12 & VTP-LT-205 & $\begin{array}{l}\text { Glycol Expansion Tank Level } \\
\text { Transmitter }\end{array}$ & $\begin{array}{l}\text { Drexelbrook } \\
\text { Xmtr: } 408-8202-001 \\
\text { Probe: } 270-1-968\end{array}$ & $0-100 \%$ & LAL-205 & $50 \%$ \\
\hline 13 & VTP-LT-185 & Seal Pot Level Transmitter & $\begin{array}{l}\text { Drexelbrook } \\
\text { Xmtr: 408-8232-001 } \\
\text { Probe: }\end{array}$ & $0-100 \%$ & $\begin{array}{l}\text { LAH-185 } \\
\text { LAL-185 }\end{array}$ & $\begin{array}{l}80 \% \\
30 \%\end{array}$ \\
\hline 14 & VTP-TS-206 & Glycol Temperature Switch & & $70^{\circ} \mathrm{F}$ & $N / A$ & N/A \\
\hline 15 & VTP-TI-176 & Inlet Temperature Indicator & Wilkerson DIS474 & Type J & $N / A$ & N/A \\
\hline 16 & VTP-PDI-177 & $\begin{array}{l}\text { Heater/Demister Differential Pressure } \\
\text { Indicator }\end{array}$ & Wilkerson DIS471 & $0-2^{\prime \prime}$ WC & $N / A$ & N/A \\
\hline 17 & VTP-PDI-178 & Prefilter Differential Pressure Indicator & Wilkerson DIS471 & $0-2^{\prime \prime}$ WC & $N / A$ & N/A \\
\hline 18 & VTP-TI-179 & $\begin{array}{l}\text { HEPA Filter \#1 Inlet Temperature } \\
\text { Indicator }\end{array}$ & Wilkerson DIS474 & Type J & $\mathrm{N} / \mathrm{A}$ & N/A \\
\hline 19 & VTP-PDI-180 & $\begin{array}{l}\text { HEPA Filter \#1 Differential Pressure } \\
\text { Indicator }\end{array}$ & Wilkerson DIS471 & $0-10^{\prime \prime} W C$ & N/A & N/A \\
\hline
\end{tabular}


Title: Portable 1,000 CFM Exhausters System Design Description (SDD)
DI: HNF-2934

Rev. 0

\begin{tabular}{|c|l|l|l|c|c|c|}
\hline Item & \multicolumn{1}{|c|}{ Tagname } & \multicolumn{1}{|c|}{ Description } & \multicolumn{1}{|c|}{$\begin{array}{c}\text { Manufacturer \& Model } \\
\text { No. }\end{array}$} & $\begin{array}{c}\text { Range } \\
\text { Or Setpoint }\end{array}$ & \multicolumn{1}{c|}{$\begin{array}{c}\text { Alarms } \\
\text { Setpoints }\end{array}$} \\
\hline 20 & VTP-PDI-181 & $\begin{array}{l}\text { Filter Train Differential Pressure } \\
\text { Indicator }\end{array}$ & Wilkerson DIS471 & $0-6$ " WC & N/A \\
\hline 21 & VTP-PDI-182 & $\begin{array}{l}\text { HEPA Filter \#2 Differential Pressure } \\
\text { Indicator }\end{array}$ & Wilkerson DIS471 & $0-6$ WC & N/A \\
\hline 22 & VTP-TI-183 & Stack Temperature Indicator & Wilkerson DIS474 & Type J & N/A \\
\hline 23 & VTP-FI-184 & Stack Flow Indicator & Wilkerson DIS471 & $0-1194$ SCFM & N/A & N/A \\
\hline 24 & VTP-LI-205 & Glycol Expansion Tank Level Indicator & Wilkerson DIS471 & $0-100 \%$ & N/A & N/A \\
\hline 25 & VTP-LI-185 & Seal Pot Level Indicator & Wilkerson DIS471 & $0-100 \%$ & N/A & N/A \\
\hline
\end{tabular}


Title: Portable 1,000 CFM Exhausters System Design Description (SDD)

DI: HNF-2934

Rev. 0

\subsection{CONTROLS}

The following table describes the skid controls and lists each device by tagname.

TABLE 2.2 - SKID CONTROLS

\begin{tabular}{|c|l|l|}
\hline Item & \multicolumn{1}{|c|}{ Tagname } & \multicolumn{1}{c|}{ Description } \\
\hline 1 & VTP-PB-101 & Exhaust Fan Start Pushbutton \\
\hline 2 & VTP-PB-102 & Exhaust Fan Stop Pushbutton \\
\hline 3 & VTP-HS-103 & Exhaust Fan Off/Enable Selector Switch \\
\hline 4 & VTP-HS-101 & Seal Pot Pump Hand/Auto Key Switch \\
\hline 5 & VTP-HS-102 & Glycol Pump On/Off Selector Switch \\
\hline 6 & VTP-SS-102 & Auto Pump 1/2 Control Selector Switch \\
\hline 7 & VTP-PB-104 & CAM Bypass Pushbutton \\
\hline
\end{tabular}

\subsection{USER CHARACTERISTICS}

The exhaust skid, while in the primary tank ventilation system backup role for $\mathrm{C}-106$ and AY-102, is intended to be controlled locally by an operator. The operator will start and stop the exhaust fan under administrative control. An instrument technician or engineer will be required to change values in the PLC's data tables depending upon the desired mode of operation. This will be done locally by connecting a laptop loaded with the appropriate PLC programming software to the skid PLC. The technician or engineer will then make the appropriate data table changes and verify that the appropriate mode of operation has been selected. This will also require the examination of certain data table fields.

Since an $\mathrm{HMI}$ is not being utilized for control purposes, passwords are not required and all security access issues must be addressed in the administrative procedures.

\section{SPECIFIC REQUIREMENTS}

\subsection{PROCESS REQUIREMENTS}

The exhauster skid shall be able to exhaust 1000 SCFM from the waste tank dome space on a continuous basis. The High Efficiency Mist Eliminator is provided to remove any entrained moisture immediately upon reaching the exhauster. The glycol heater is provided to raise the relative humidity of the air by heating it before it is introduced to the HEPA filters. The HEPA filters trap any airborne contamination before the tank air is exhausted to the atmosphere. 
Title: Portable 1,000 CFM Exhausters

System Design Description (SDD)
DI: HNF-2934

Rev. 0

\subsection{INTERFACE REQUIREMENTS}

For the W-320 backup function, the exhauster skid will operate as a stand-alone unit and is not required to interface remotely with any of the tank farm SCADA systems, such as, TMACS, CASS or the MPSS.

A local operator interface is required to allow the operator to operate the exhauster skid, including changing operational parameters and acknowledging alarms.

\section{REFERENCES}

4.1 "HNF-2686 ATP For A Continuous Use, Portable 1000 CFM Exhauster POR-007-SKID E"

4.2 "HNF-2687 ATP For A Continuous Use, Portable 1000 CFM Exhauster POR-008-SKID F"

\section{DESCRIPTION OF PLC CONTROL AND PROGRAM}

\subsection{GENERAL}

The PLC program controlling exhauster operation has three operational modes. These modes differ in the process variable that is used to provide feedback to the variable speed drive for the fan motor. Changing modes is accomplished by changing the value of two digital values in the processor. When a new mode is selected, portions of the PLC program that are required for the mode are enabled and the appropriate values are copied to alarm setpoint registers.

There are two basic modes of control for the skid: Flow Control Mode (see Section 5.2) and Pressure Control Mode (see Section 5.3). If Flow Control Mode is to be used then PDT-170B, mounted on the exhauster inlet, must be connected to the appropriate transmitter connection port. If the Pressure Control Mode is to be used then PDT-170A, mounted on the exhauster fan inlet, is connected to the same port instead. This way only one of these two transmitters is connected to the PLC at any one time. Verification that the correct one is plugged in must be done as part of the setup procedure for the skid depending on the desired mode of operation.

Following are descriptions of each mode of control and the PLC register changes required to start each mode.

\subsection{FLOW CONTROL MODE}

Flow Control mode should be used when the exhauster is connected to a primary tank space where it is desired to maintain a relatively high flow under a light vacuum load.

In this mode, the fan speed is controlled to maintain the flow rate at the value specified in the PLC floating register F8:6. This value is entered in SCFM and is currently set to 1000. PID control is utilized to modulate the control signal to the variable speed drive in order to achieve the desired setpoint. A deadband is used to avoid oscillation around the setpoint. 
Title: Portable 1,000 CFM Exhausters System Design Description (SDD)
Dl: HNF-2934

Rev. 0

Enter the Flow Control Mode of operation by setting bits B3/0 and B3/1 to zero (0). PDT-170B must be installed and connected to the PLC instead of PDT-170A for this mode to operate. PDT-170B is mounted on the exhauster inlet.

\subsection{PRESSURE CONTROL MODE}

Pressure Control mode should be used when the exhauster is connected to a double-shell tank annulus ("Dry Annulus Operation"). (CAUTION: The Pressure Control Mode is not intended and should never be used for ventilating the primary tank space.)

This mode uses the fan inlet pressure from transmitter PDT-170A as feedback to the control routine. The pressure/vacuum setpoint is entered in PLC floating register F8:7. The value is entered in inches of water column, and is currently set to $12^{\prime \prime}$ WC. (CAUTION: Values greater than $13.75^{\prime \prime}$ WC will cause the seal pot contents to flow into the plenum.) PID control is utilized to modulate the control signal to the variable speed drive in order to achieve the desired setpoint. A deadband is - used to avoid oscillation around the setpoint.

Enter this mode of operation by setting bits B3/0 to one (1) and B3/1 to zero (0). PDT-170A must be installed and connected to the PLC instead of PDT-170B for this mode to operate. Some alarm setpoints are adjusted to take account for the change in instrumentation and operating conditions. Alarm setpoints used with this mode are marked with $(V)$ in Table 2.1.

\subsection{HIGH-VACUUM MODE}

High-Vacuum mode should be used only when the seal pot drains inside the filter housing have been suitably plugged, when it is intended to operate the exhauster at a maximum vacuum to increase throughput.

High-vacuum mode is designed to maximize exhauster throughput and inlet vacuum by setting the variable speed drive at a maximum output value. PID control is bypassed, and the normal output register of the PID instruction is set to 16384 (maximum).

Enter this mode of operation by setting bits B3/0 and B3/1 to one (1). PDT-170A must be installed and connected for this mode to operate. Some alarm setpoints are adjusted to take account for the change in instrumentation and operating conditions. Alarm setpoints used with this mode are marked with (HV) in Table 2.1.

\subsection{OPERATIONAL INTERLOCKS}

Fan operation interlocks are a subset of the alarms list in Table 2.1. Some alarms shut down the system immediately, while others shut down the system only after the interlock alarm is true for a preset amount of time. Usually only one interlock alarm is required to shut down the system, but in some cases a combination of interlock alarms is required.

Interlock alarm tags and applicable time delays are shown in Table 5.1. 
Title: Portable 1,000 CFM Exhausters System Design Description (SDD)
DI: HNF-2934

Rev. 0

TABLE 5.1 - INTERLOCKS

\begin{tabular}{|c|c|c|c|}
\hline Item & Alarm Tag & Description & Delay (sec) \\
\hline 1 & ROC-180 & FILTER 1 DP ROC & 3 \\
\hline 2 & PAL-170B & PLENUM 1 PRESS LO & 0 \\
\hline 3 & FAL-184 & STACK FLOW LO & 30 \\
\hline 4 & FAH-184 & STACK FLOW HI & 15 \\
\hline 5 & PDAL-180 & FILTER 1 DP LO & N/A \\
\hline 6 & PDAHH-180 & FILTER 1 DP HI HI & N/A \\
\hline 7 & PDAL-182 & FILTER 2 DP LO & N/A \\
\hline 8 & PDAHH-182 & FILTER 2 DP HI HI & N/A \\
\hline 9 & PDAL-181 & FILTER 1\&2 DP LO & N/A \\
\hline 10 & PDAHH-181 & FILTER 1\&2 DP HI HI & N/A \\
\hline 11 & PAH-170A & INLET VACUUM HI & 5 \\
\hline 12 & LAH-185 & SEAL POT LEVEL HI & N/A \\
\hline 13 & LAL-185 & SEAL POT LEVEL LO & N/A \\
\hline 14 & PAH-170B & PLENUM 1 PRESS HI & 10 \\
\hline 15 & ROC-182 & FILTER 2 DP ROC & 3 \\
\hline
\end{tabular}


HNF -2934

Rev. 0

This page intentionally left blank 
Title: Portable 1,000 CFM Exhausters

System Design Description (SDD)
DI: HNF-2934

Rev. 0

APPENDIX A - ALLEN-BRADLEY (A-B) SLC500 PLC PROGRAM PRINTOUT

Page A-O 
Number of Program Files:21

Narne

File sub-Progran Description

\begin{tabular}{|c|c|c|}
\hline & 0 & [SYSIEM DATA SICRAGE HEADER] \\
\hline & 1 & [RESTRVED ARFA] \\
\hline MATN & 2 & MANPCES THE EXECTMTON OF SURPOUTIIES \\
\hline STRRTUP & 3 & RUN CN FOWER-UP TO INETLAITZS BIT/WCRD STATUS \\
\hline DFPUIDATA & 4 & INEUT FIIE TRANSFER TO DATAGRAM (NEO) + WW TRANS? \\
\hline INIRLKS & 5 & FAN CONIROL INTERLOCKS \\
\hline FPIKNIRL & 6 & AIR FLOW PID FAN ONIRL \\
\hline MESGVIEW & 7 & HANDIES I/O BETWESN MESSAGE VIEN TEFMMALS AND STC \\
\hline SISSTAT & 8 & RETUFNS UNACK ATM SIYIIUS AND MOTCR CONTACT ON/OFF \\
\hline STMUATE & 9 & SEIS EPN ON BIIS AND TURNS OFF DAHACRAM TRANSEDR \\
\hline GYYOOLFMP & 10 & GLOOT PUMP CONIROLS AND INIERLOCKS \\
\hline ERRSTAT & 11 & SHIUS DONN MOTOR ON MAYOR FRLIT \\
\hline CANIER & 22 & CONIERS FOR SYSTEM STATUS CPANLS? \\
\hline FIFO & 13 & ENABLES AND LOADS FIFO BUFFERS \\
\hline CONIER & 14 & SYSTEM STATUS OCANIERS \\
\hline CLRFALIT & 15 & CEAR ERRCORS ON PONER-UP \\
\hline DNIL & 16 & MDRE INIERLOCSS FCR FILE 5 \\
\hline copx_our & 17 & COEY DAIA TO OULFUL MDDULS \\
\hline FIFCBOEFER & 18 & LOMLS FIFO EUIFERS USED IN RAIE OF OHANGE CALCS \\
\hline SFAIFOMPMP & 19 & SFAI, ROT FIMP ONIROL \\
\hline FANONIRL & 20 & INUET FRESSURE FAN CONIFU \\
\hline
\end{tabular}




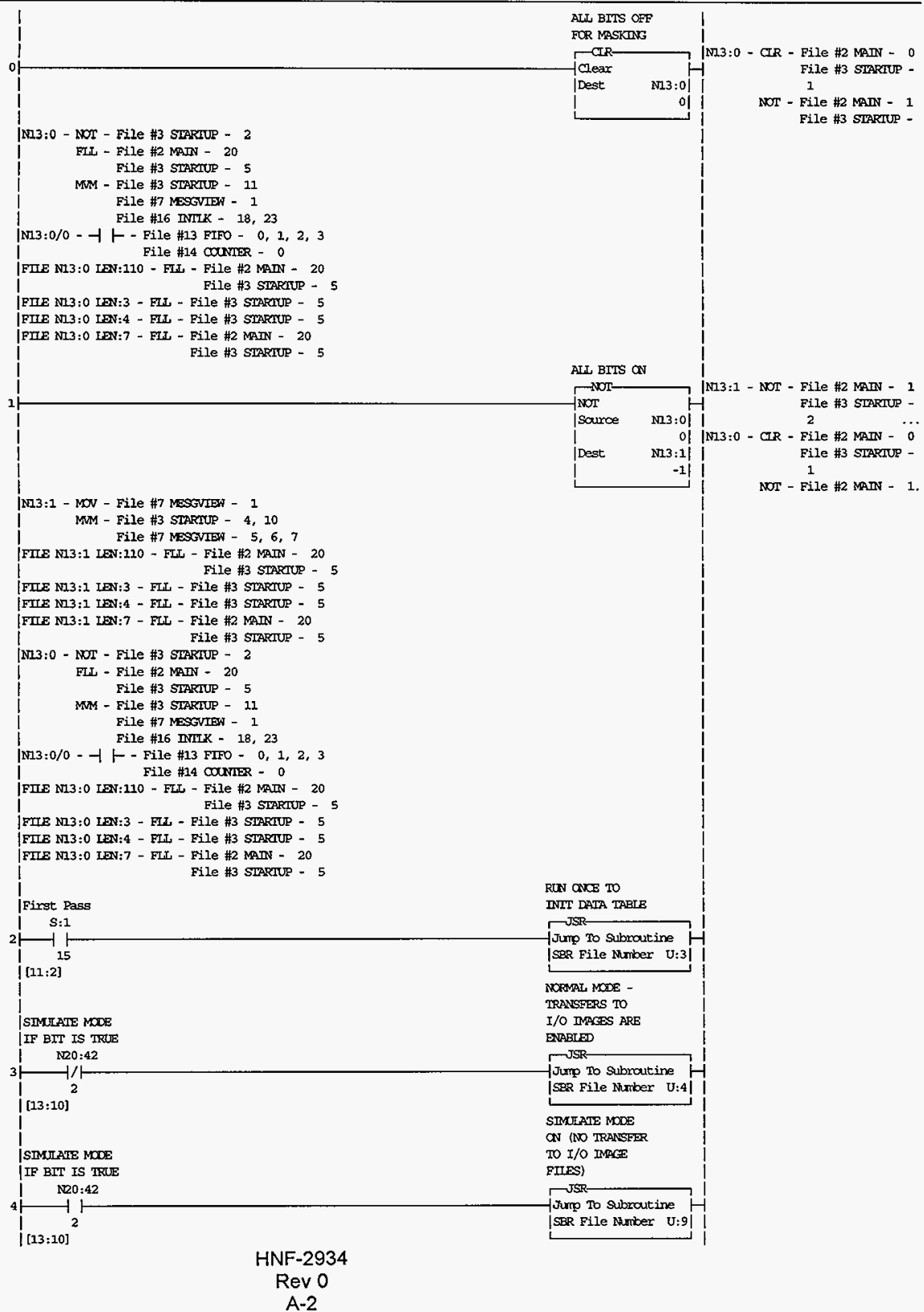




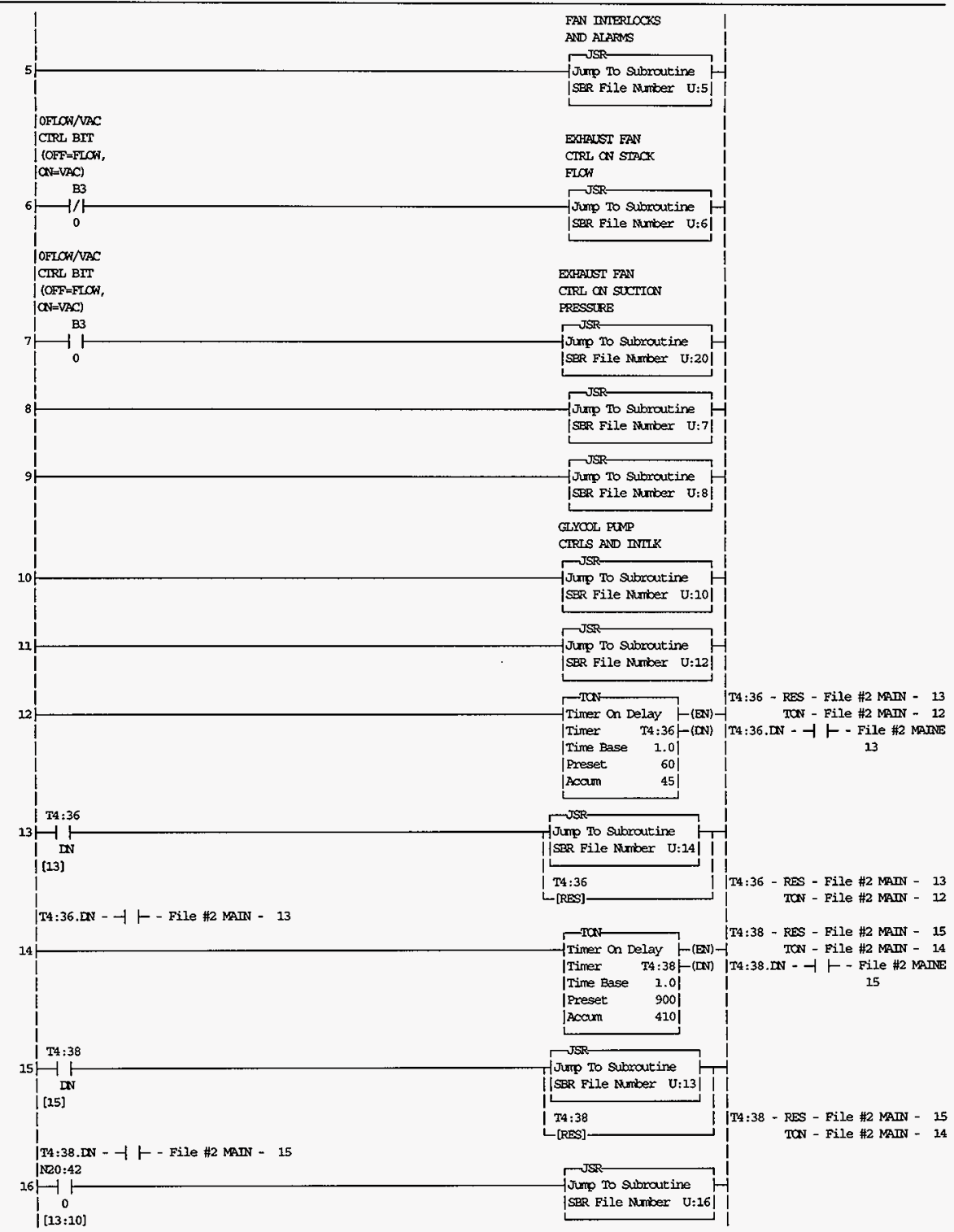




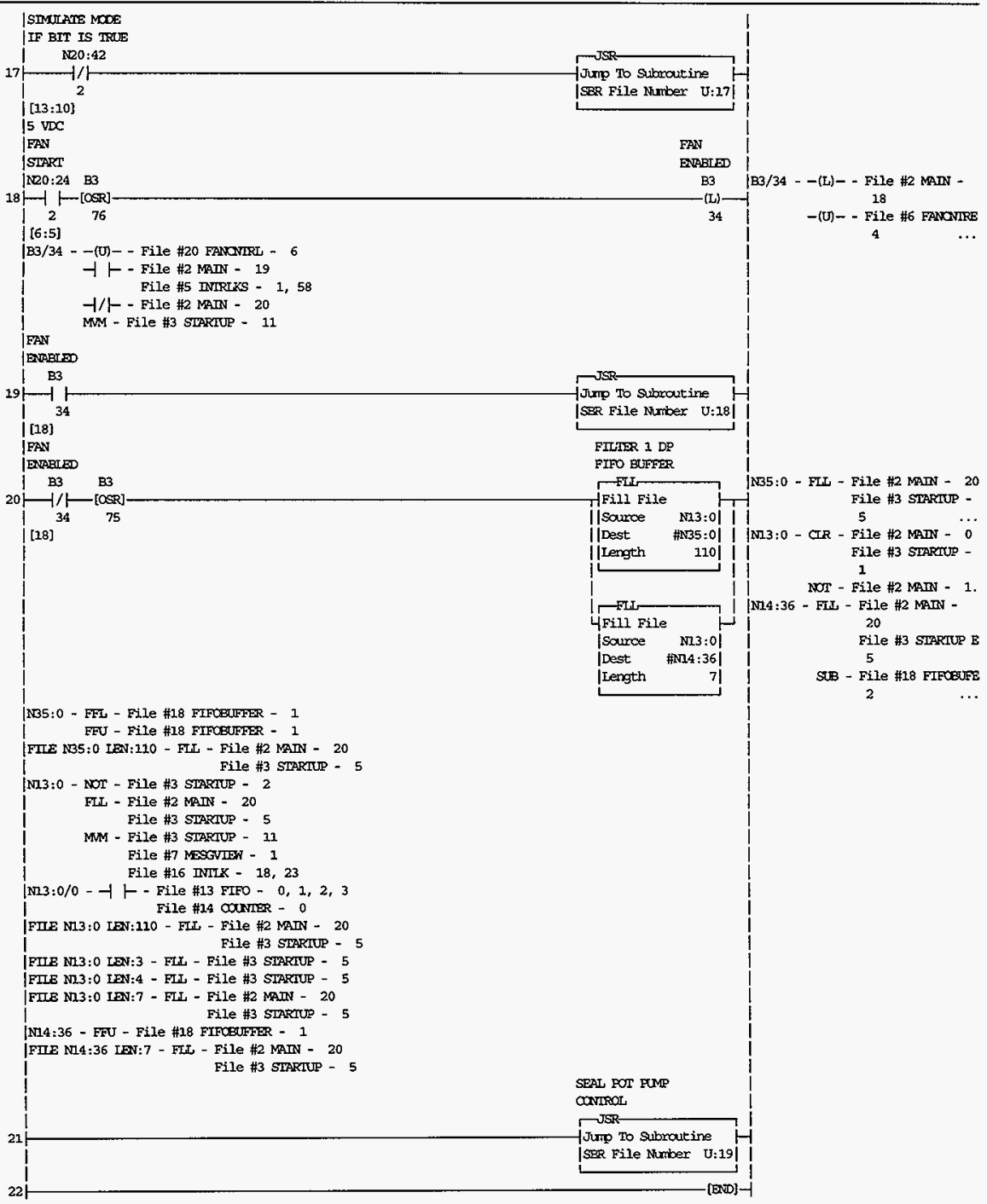




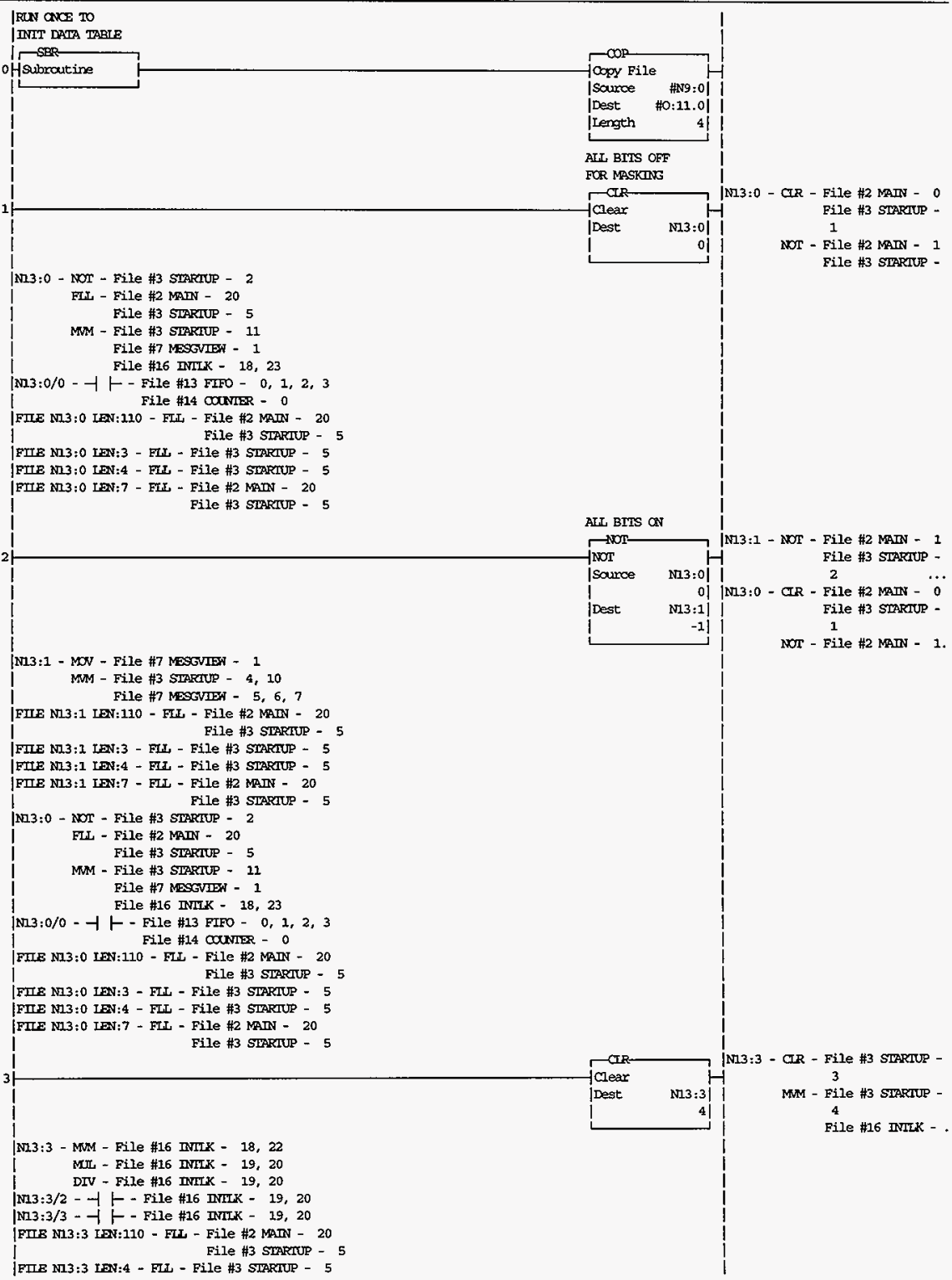

HNF-2934 


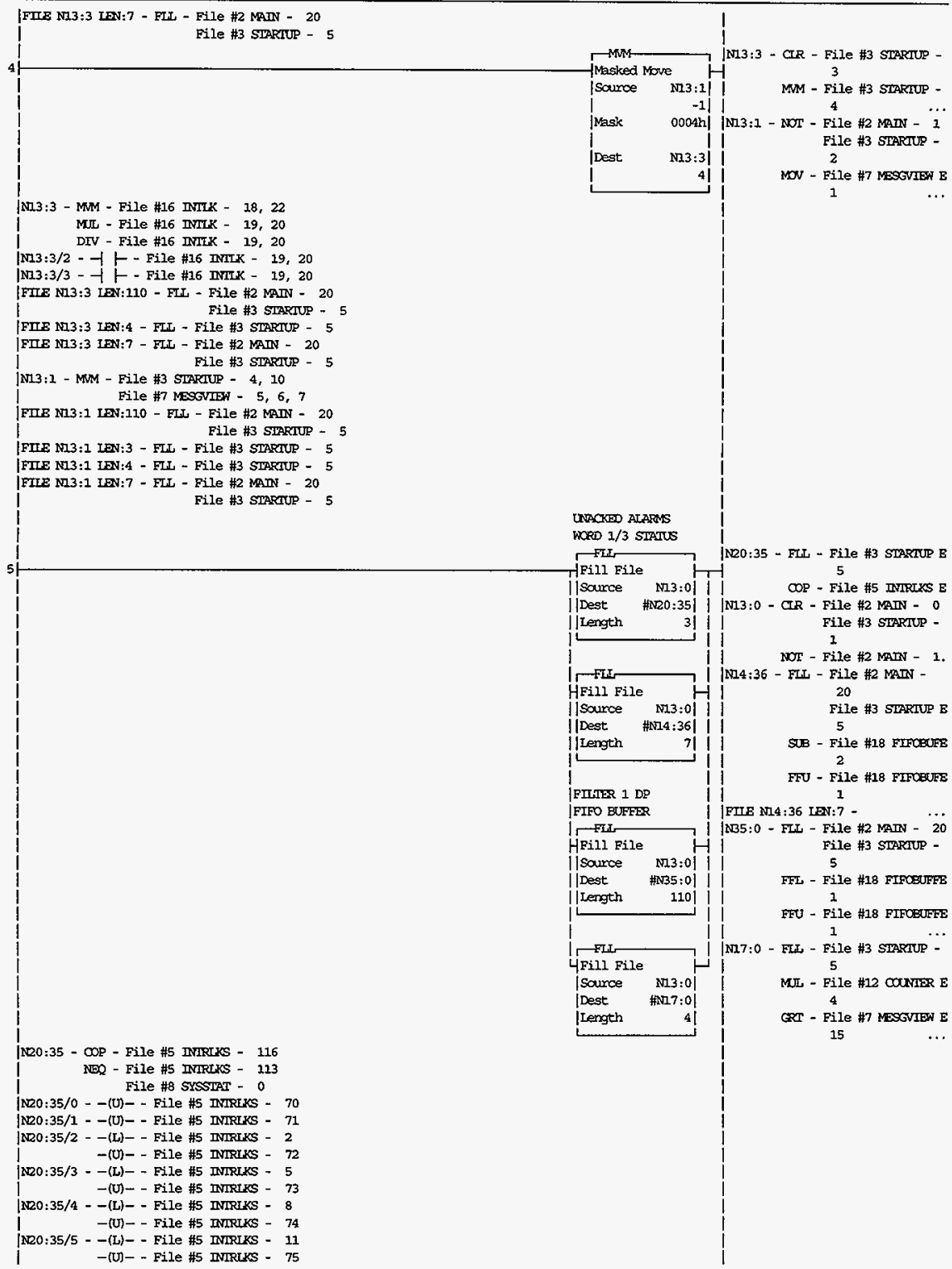

HNF-2934

Rev 0 
|Ne0:35/6 - - (L) - - File \#5 INTRUkS - 14 -(U) - - File \#5 INIRLKS - 76 $-1 / \vdash-$ File \#5 INIRUKS - 15
IN20:35/7 - - (L) - File \#5 INIRLKS - 16

$1 \quad$-(U) - - File \#5 INIRLKS - 77

|ND0:35/8 - -(L) - - File \#5 INIRLXS - 17

- (U) - - File \#5 BNLLKS - 45, 78

N20:35/9 - -(L) - - File \#5 INIRIKS - 19

-(U) - - File \#5 INIRtiks - 79

$-1 / 1-$ File \#5 MULLKS - 20

IN20:35/10 - -(L) - File \#5 IMREKS - 21

1 $-(\mathrm{U})$ - - File \#5 IMRRKS - 80

Ne0:35/11 - -(L)- - File \#5 INLRLS - 22

| $-(U)$ - - File \#5 INIRIXS - 46, 81

|N20:35/12 - -(L) - - File \#5 INIRLKS - 24

| $-(\mathrm{U})$ - - Fille \#5 INTRIXS - 82

1 - $1 /$ - File \#5 INIRLKS - 25

jN20:35/13 - -(L) - Eile \#5 INLRLKS - 26

-(U) - - File \#5 INIRLKS - 83

N20:35/14 - - (U) - - File \#5 INIRIKS - 8A

N20:35/15 - -(L)- - File \#5 INIRIKS - 27

-(U) - - File \#5 INIRIKS - 85

$-1 /$ - File \#10 GroOLPMP - 0,1

FILE NQ0:35 IEN:100 - FEL - File \#13 FIFO - 10

FIIS NQ0:35 IEN:30 - FFL - File \#18 FIFOEUFFER - 1

FIIE NDO:35 LEN:3 - FLL - File \#3 SIARTUP - 5

|

NI2:0 - NOT - File \#3 STARTUP - 2

Fis - File \#2 MAIN - 20

File \#3 SIARIUP - 5

MM - File \#3 SIARTUP - 11

File \#7 MESTIIN - I

File \#16 INILK - 18, 23

N13:0/0 - $11-$ File $\# 13$ FIEO - 0, 1, 2, 3

File \#14 counise - 0

|FILE N13:0 LEN:110 - FUL - File \#2 ManN - 20

1 File \#3 STARIUP - 5

FIrE N13:0 LEN:7 - FLI - File \#2 MaN - 20

| File \#3 SIPRTUP - 5

N14:36 - FLL - File \#2 MATN - 20

File \#3 STARIUP - 5

[N35:0 - ELI - File \#2 MAMN - 20

I File \#3 STARTUP - 5

6$$
\text { File fo sLRTP - } 5
$$

G:0 - RES - File \#3 STARTUP - 6

|C5:0.AOC - LES - File \#15 CIRFAUTH - 1

ICS:0.Q - - (U) - File \#15 CLRFAILT - 0

$$
\text { | }
$$

N20:25/5 - - (L) - - File \#20 FAMONIRL - 16

-(U) - - File \#3 STARIUP - 7

MM - File \#4 INPUIDAIA - 12

File \#17 COPY our - 3

FTIE N20:25/5 LEN:100 - EFL - File \#13 FIFO - 10

JFIE NDO:25/5 LEN:30 - FFL - Eile \#18 FIFCBUFFER - 1

FIIE N20:25/5 LEN:30 - FFL - Eile \#18 FIFOBUFFER - 1

|FLLE NLO:25/5 LEN:30 - EFL - EIIE \#18 FIFOBUFFER - 1

(1)

N20:42/12 - -(U) - File \#3 SIRARTUP - 8

File \#5 INIRLKS - 68

- - File \#5 INIRIXS - 42, 121

File \#16 INILX - 17

|FIIE N20:42/12 LEN:100 - FFL - File \#13 FIFO - 10 


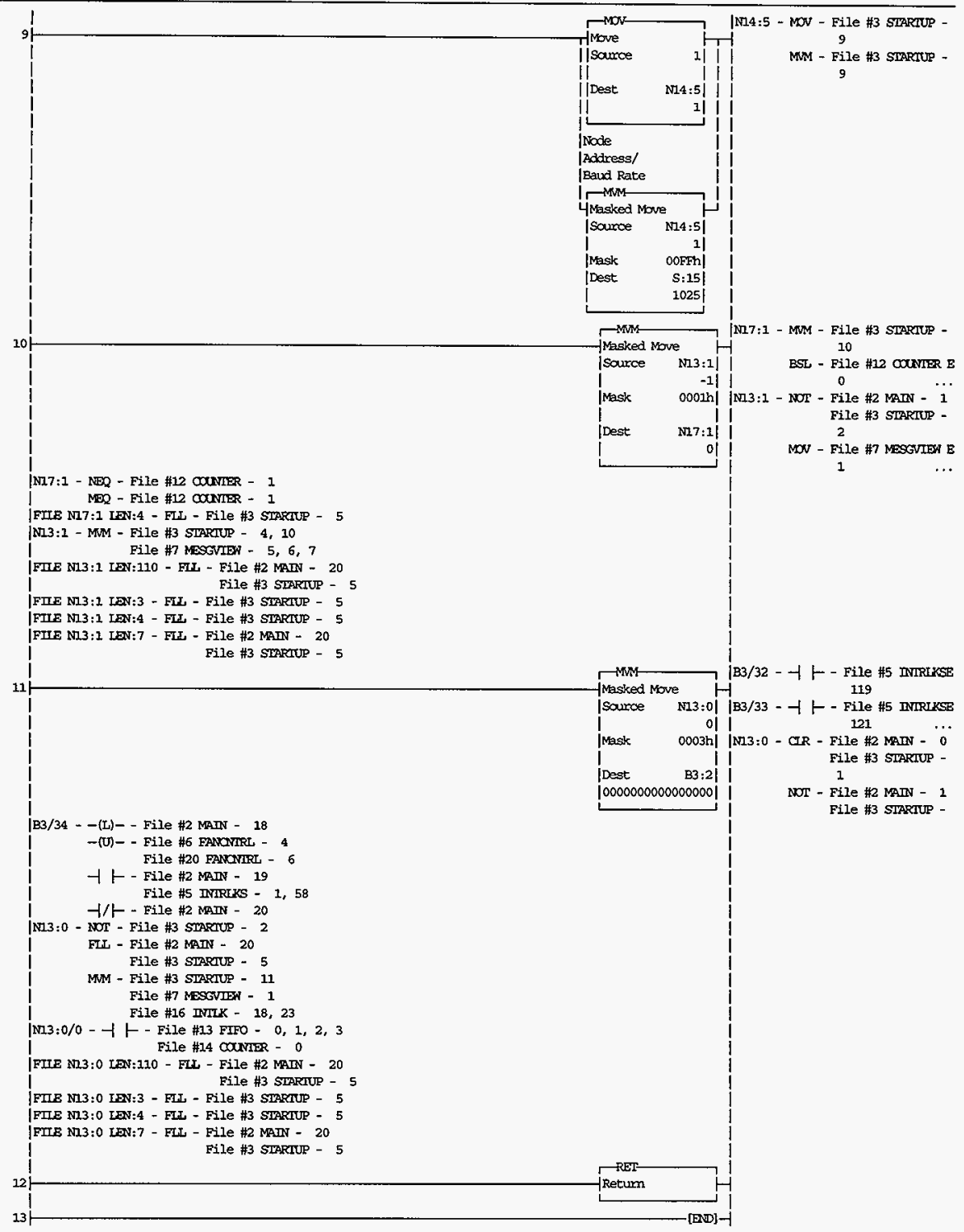




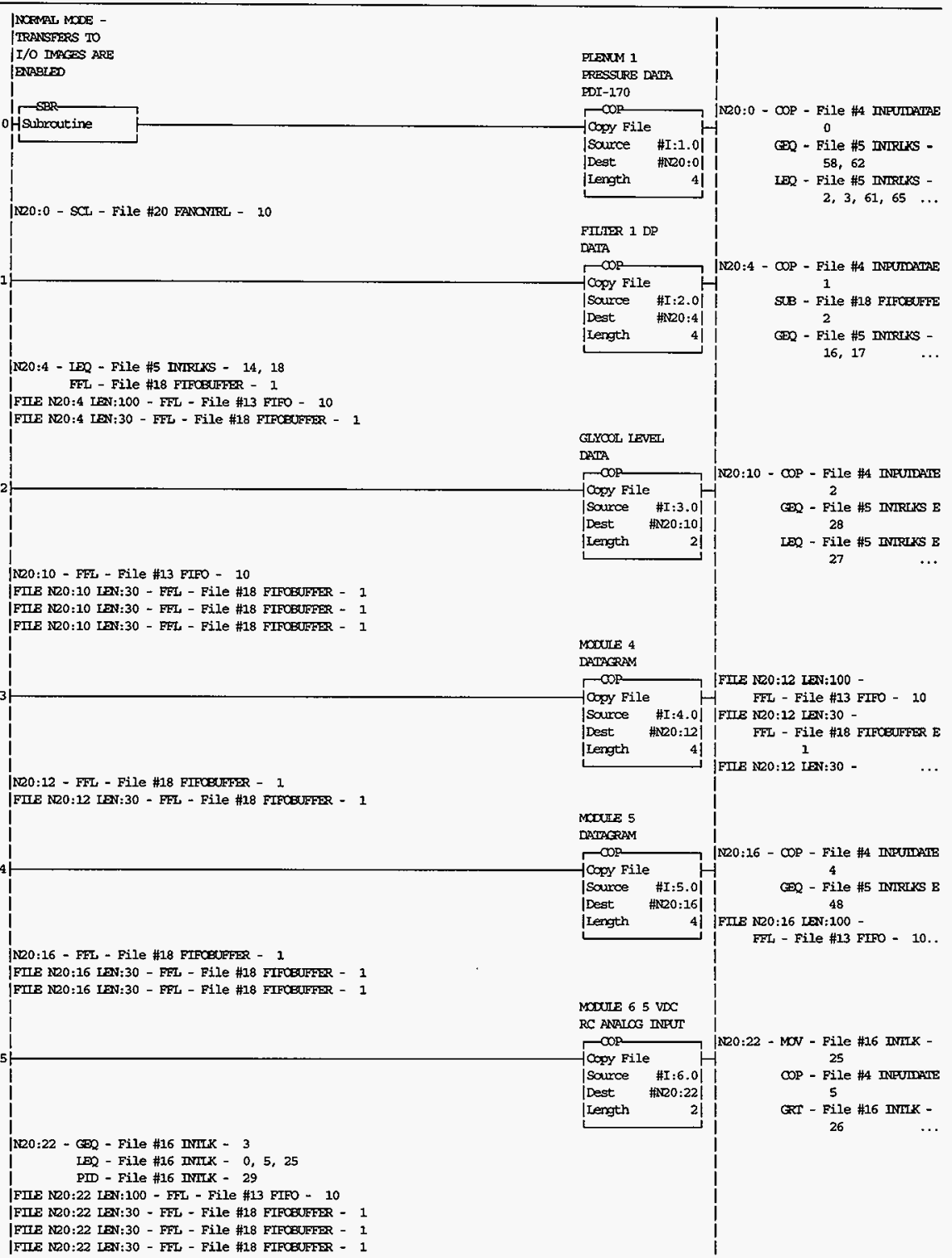




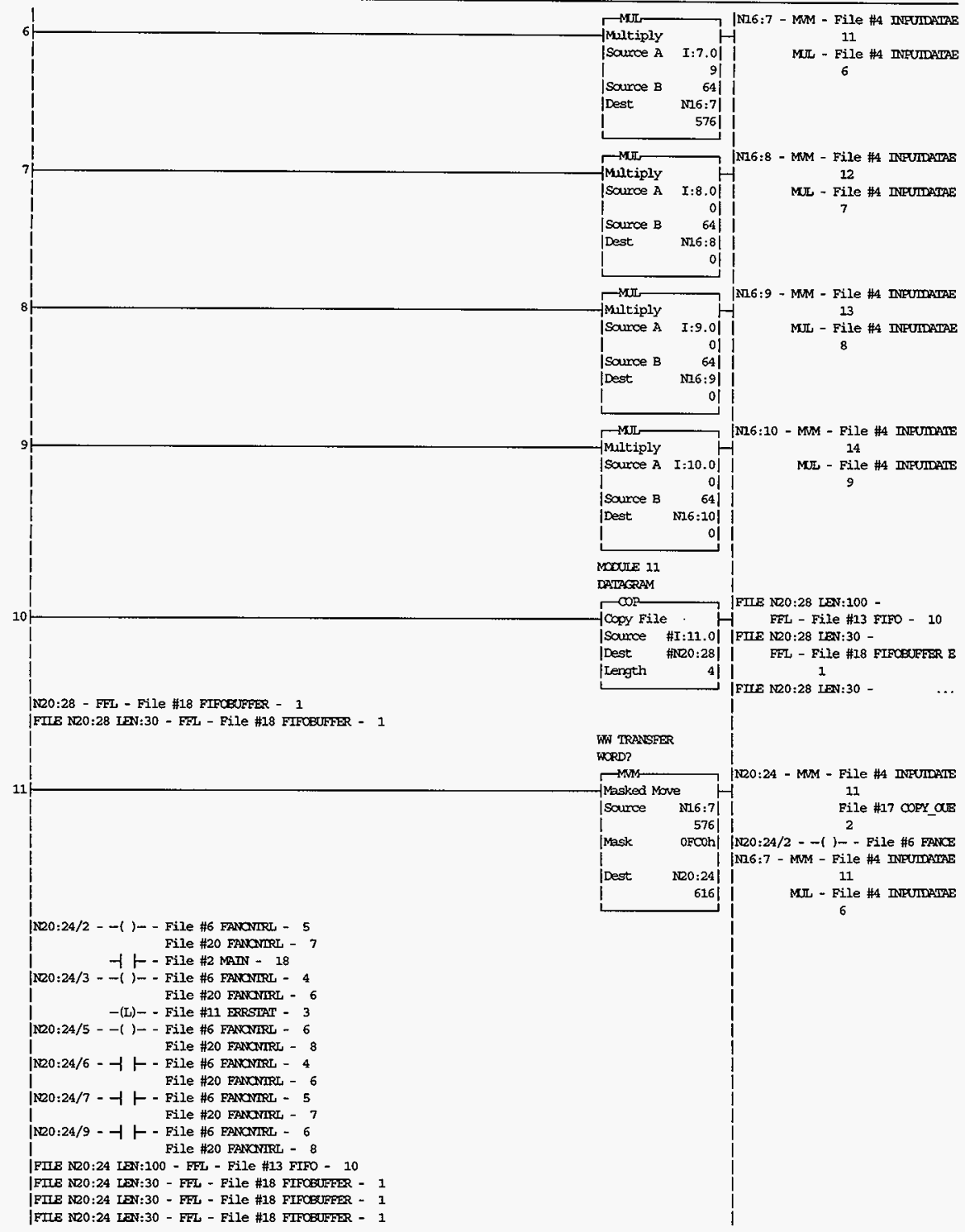




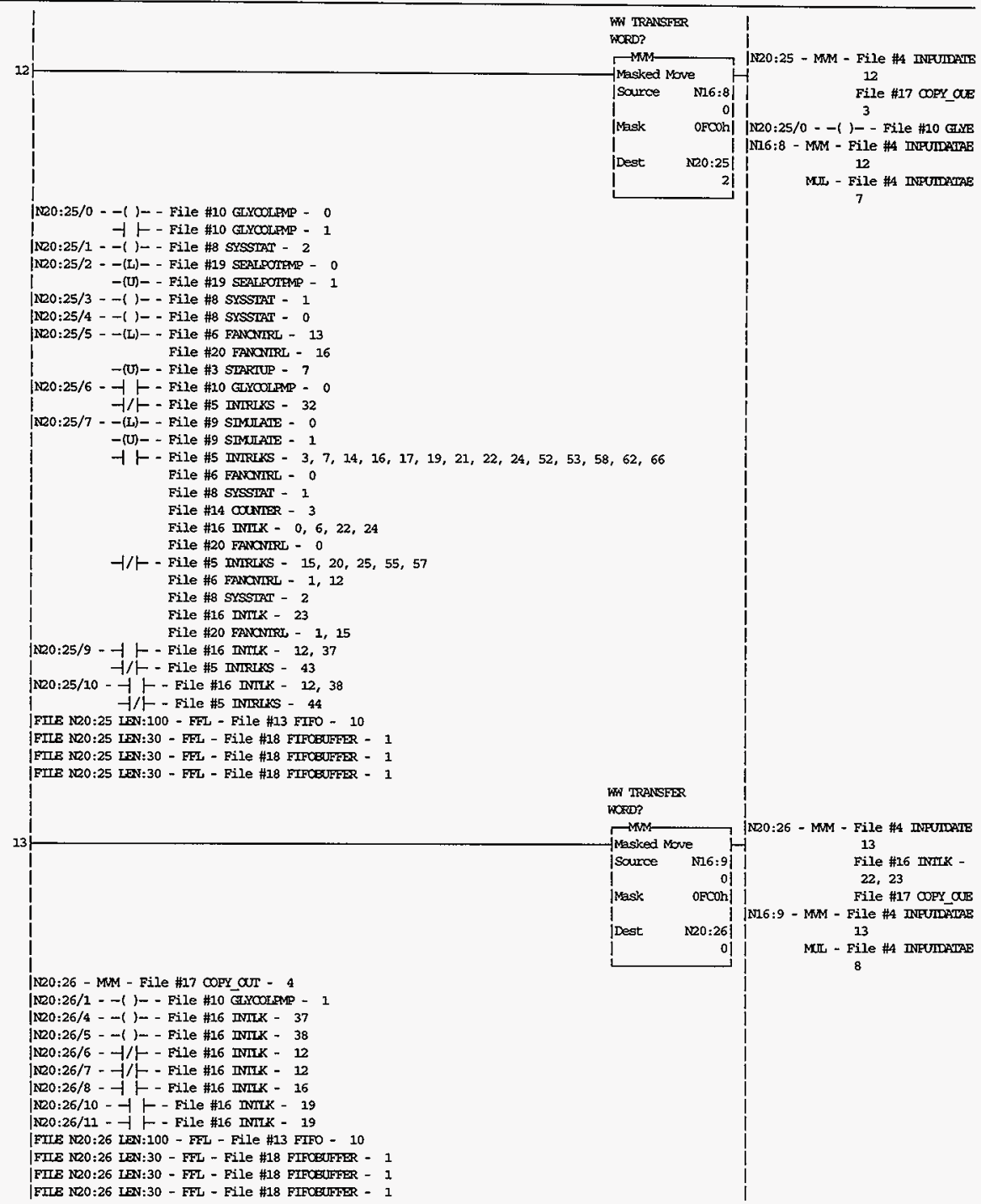




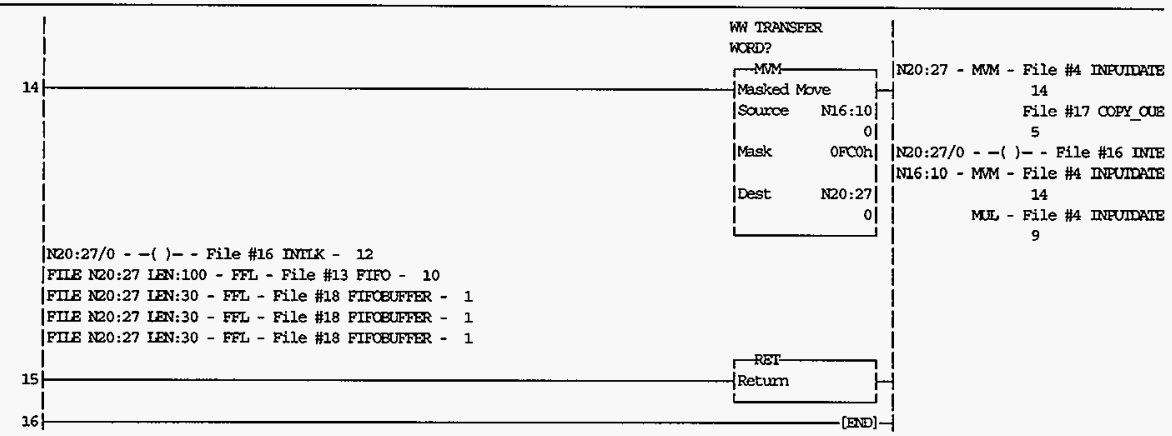




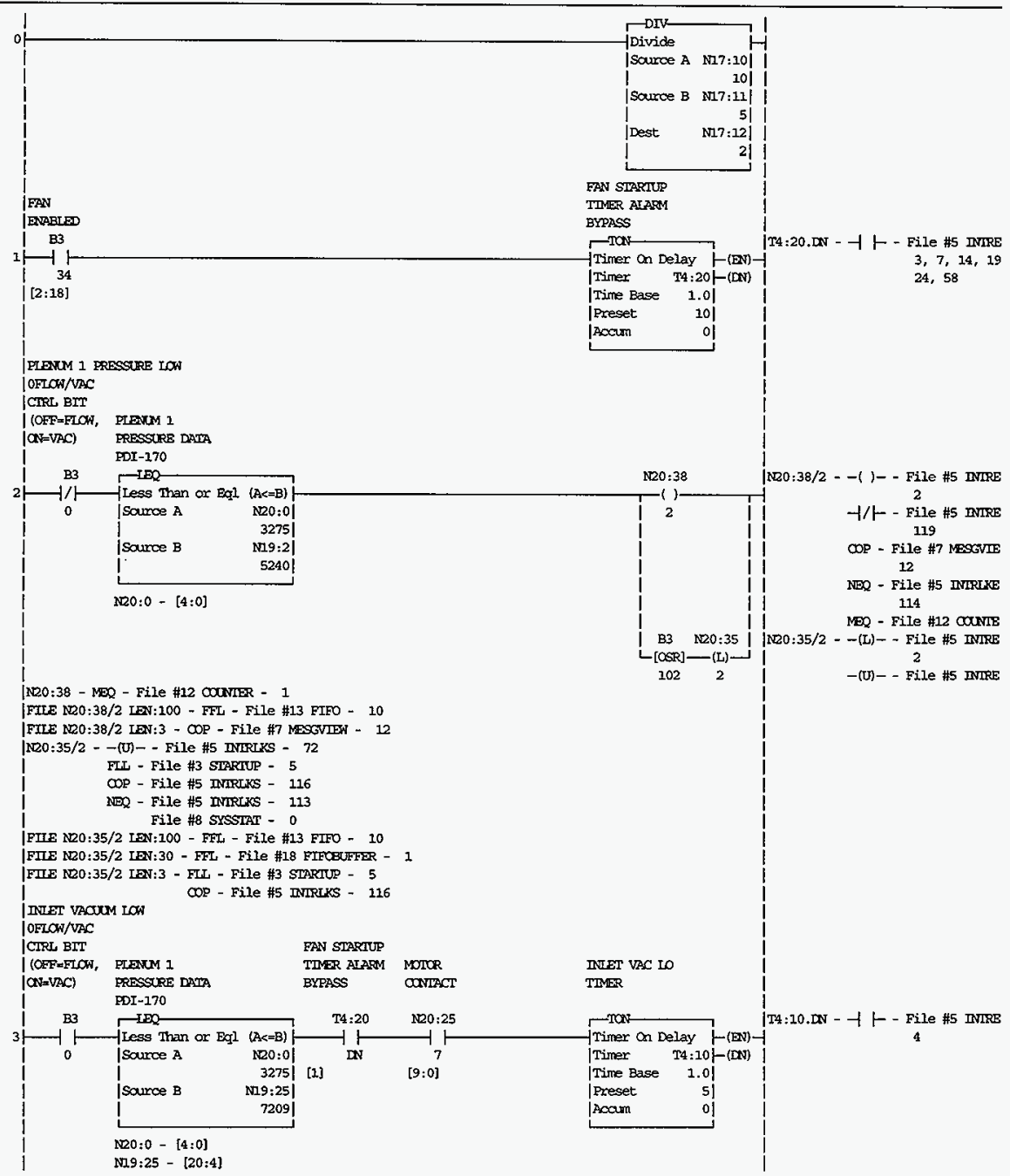




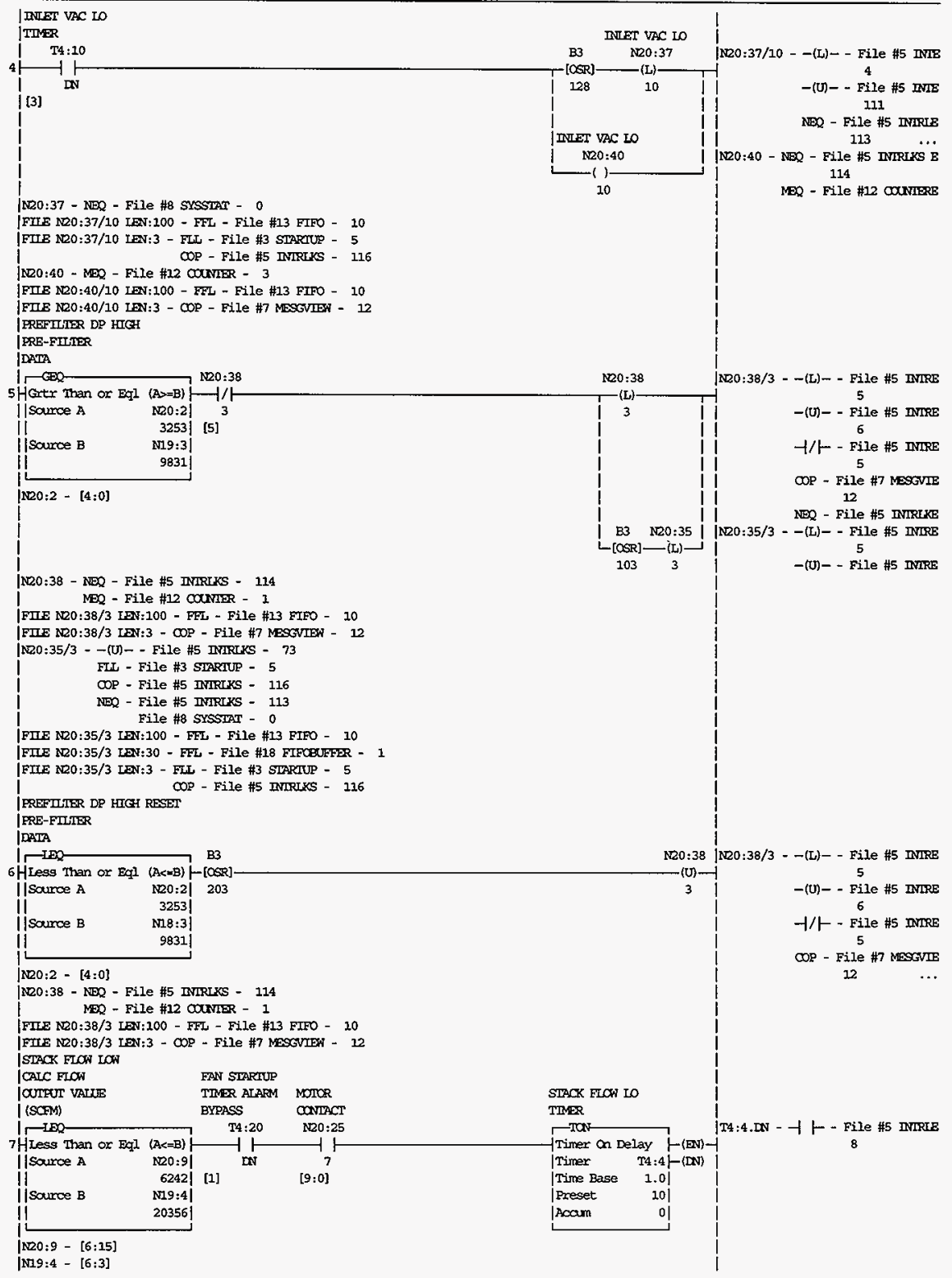




\section{SL- $\forall$ \\ $0 \wedge$ ^ə}

เع6Z-JNH

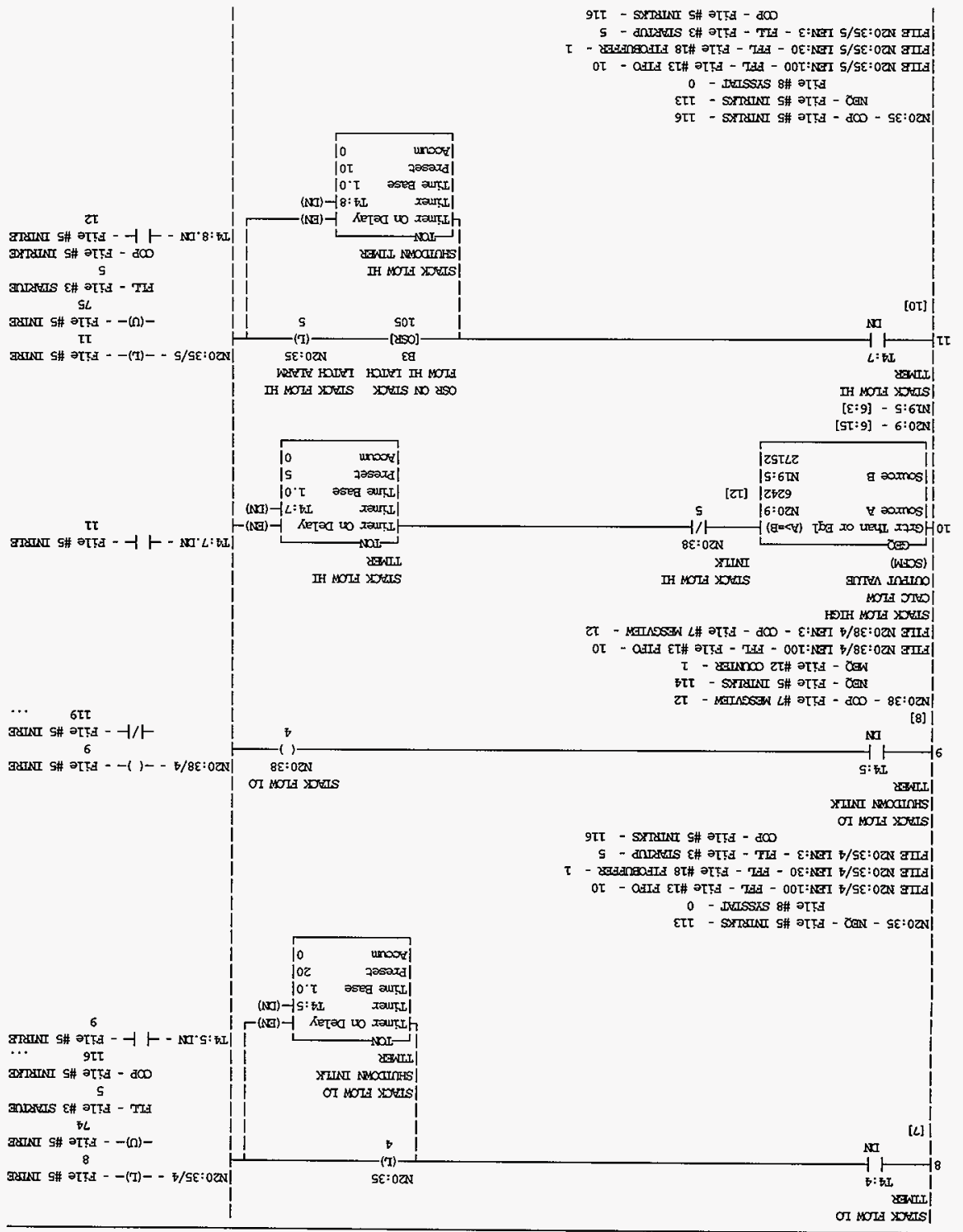




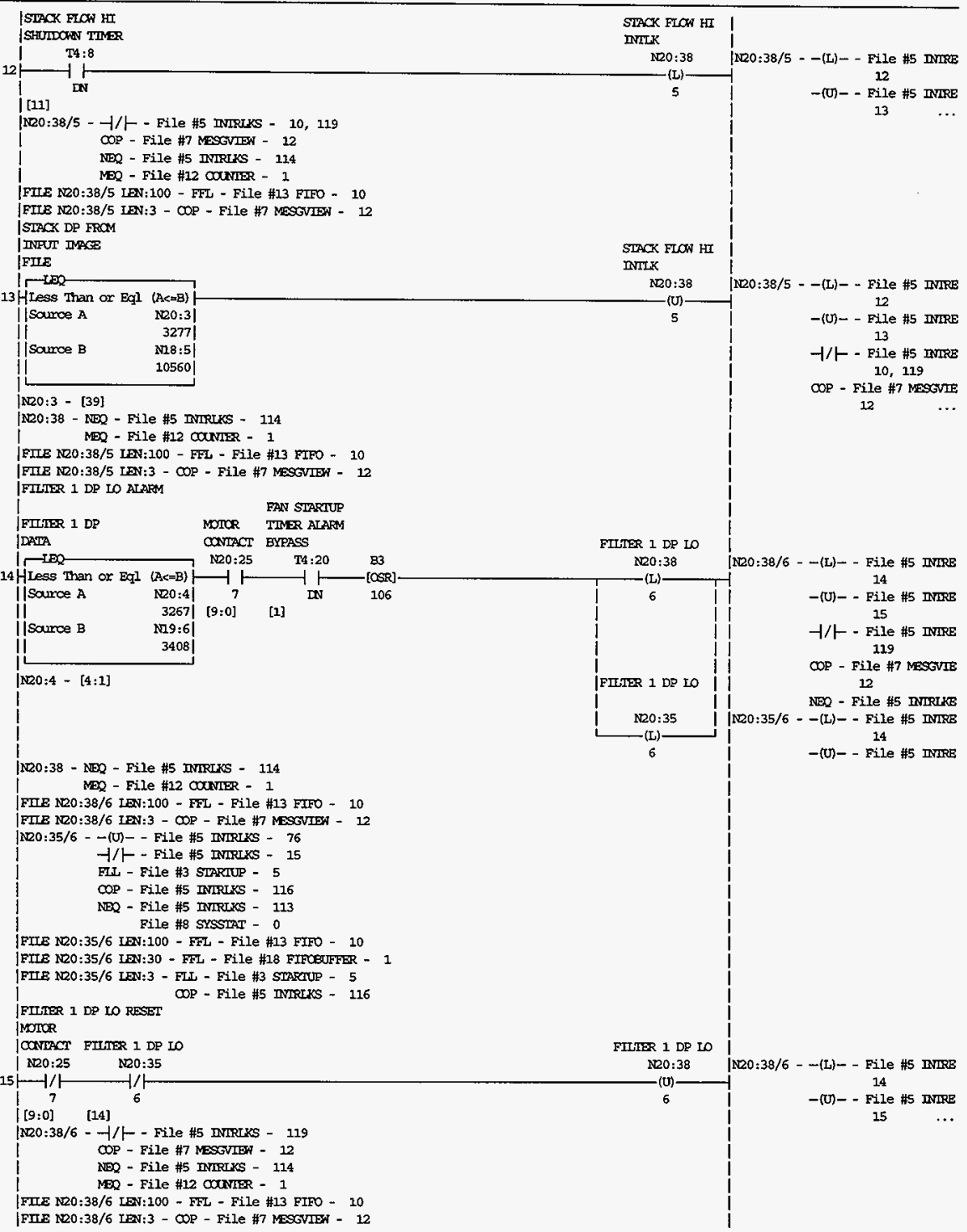




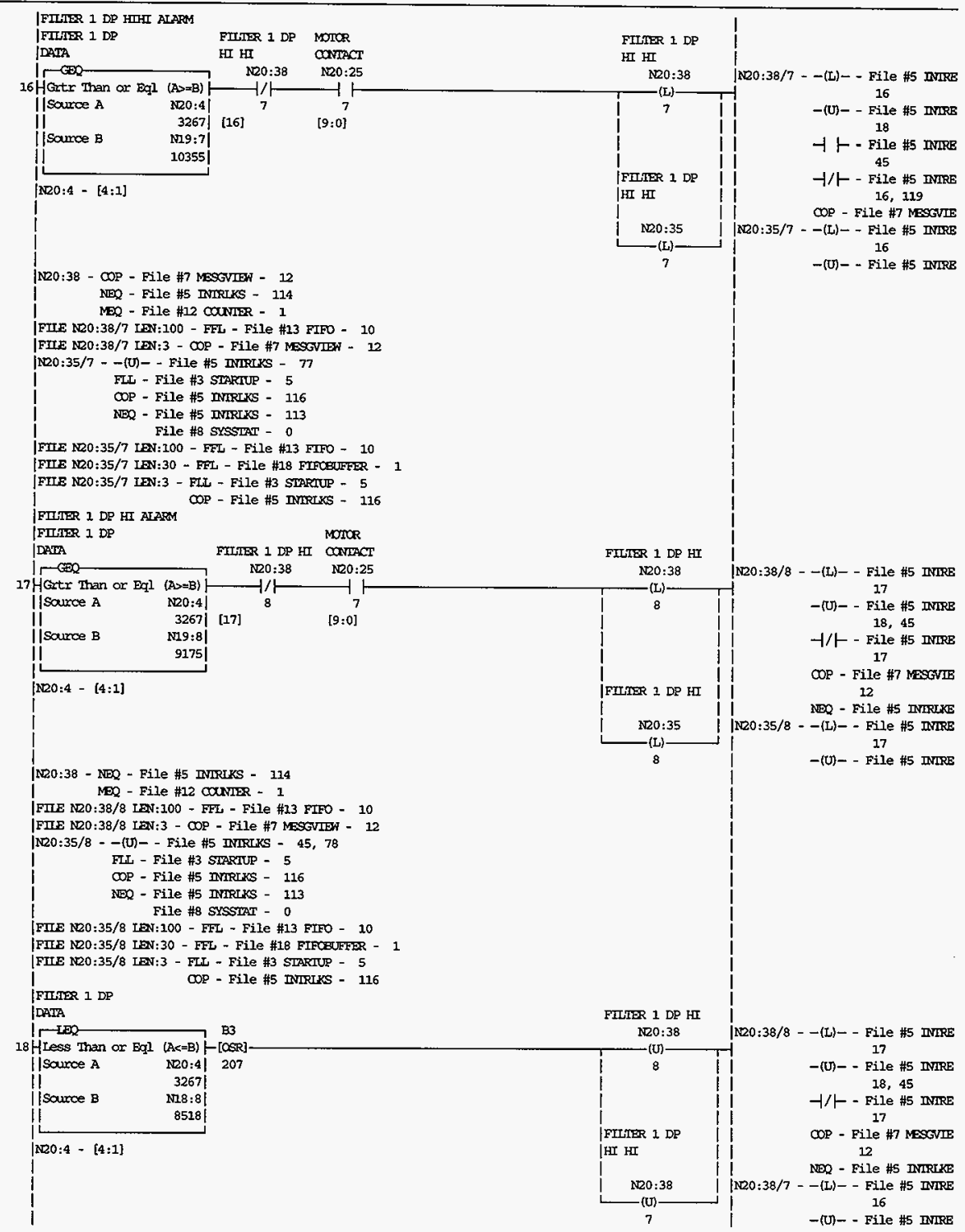


|N20:38 - NEQ - File \#5 MNRLKS - 114

MEQ - File \#12 cownitr - 1

|FIIE N20:38/8 LEN:100 - EFL - File \#13 FIFO - 10

FIIE NOO:38/8 IDN:3 - CP - File \#7 MESGVIEN - 12

N20:38/7 - - (U) - File \#S INIRUKS - 18

4 - File $\$ 5$ INIRTKS - 45

$-1 / F$ - File $\# 5$ INIRIKS - 16, 119

COP - File \#7 MESovIIA - 12

NOQ - File \#5 INIRLKS - 114

MEQ - File \#12 countrR - 1

|FIIE N20:38/7 IEN:100 - FFL - File \$13 FIFO - 10

|FIIE N20:38/7 LEN:3 - COP - File \#7 MESGVIEN - 12

FAN STARTUP

|FIITER 2 DP MOTOR TMER AIAEM

JDATA.

OANIACT EYPASS

1 IDS-

19 HIess Than or Eol $(A<=B)$

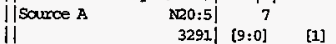

N20:25 T4:20 B3

||scarce B N9:9|

II 3495|

IN20:5 - [18:2]

N20:38 - א20 - File \#5 INIRIKS - 114

MD - File \#12 counrrs - 1

|FIIE N20:38/9 IEN:100 - FFL - File \#13 FIFO - 10

|FIIE NO0:38/9 IEN:3 - COP - File \#7 NESGIIEW - 12

|Ne0:35/9 - -(U) - - File \#5 INIRLKS - 79

- /

FIJ - File \#3 STARTUP - 5

COP - File \#5 INIRIKS - 116

NW2 - File \#5 INIRUKS - 113

File $H 8$ SYSSTAT - 0

|FIE ND0:35/9 IEN:100 - FFL - File \#13 FIFO - 10

|FIIE NE0:35/9 IEN:30 - FFL - File \#18 FIFCBUFFR - 1

|FILE N20:35/9 IEN:3 - FIX - FIle \#3 STARTUP - 5

FIITER 2 DP IO RESET

oos - File \#5 INIRIKS - 116

|MOTOR

|CONIACI FILIER 2 DP 10

$\mathrm{N} 20: 25 \quad \mathrm{~N} 20: 35$

$20 \mid-1 / 2$

[ [9:0] [19]

|N20:38/9 - - / / - File \#5 TIIRLKS - 119

( COP - File \#7 MESGVIEN - 12

NEQ - File \#5 INIRLKS - 114

NER - File \#12 COUNIER - 1

FIIE N20:38/9 IEN:100 - FFL - File \#13 ETFO - 10

|FIIE N20:38/9 IEN:3 - COP - File \#3 MESGVIEN - 12

FIITER 2 DP kI hI

FIIITR 2 DP

FIIIER \#2 DP MOTCR

DAIFA

HII $\mathrm{FII}$

CONEACT

$1-\mathrm{Can}$

N20:38

N20:25

21 Horter than or Eql

I scumce $A$

II

| Saurce B

$\{\mathrm{A}>\mathrm{B}\}$

$1 / 1$

3291] [21]

11

Source B N19:10

11360

|N20:5 - [18:2]

I

1

N20:38 - COP - File \#7 MESGVIFH - 12

NEQ - File \#5 INIRIKS - 114

MD2 - File \#12 COUNIBR - 1
FILE N20:38/10 IDN: 100 - FFL - File \#13 FIFO - 10

\section{9}

09

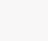


|FIIE N20:38/10 LAN:3 - COP - File \#7 MESGIDW - 12

NEO: $35 / 10$ - -(U) - - File HS INIRUKS - 80

FiL - File \#3 STPRTUP - 5

I COP - File \#5 INIRIXS - 116

NDQ - File \#5 INIRLKS - 113

File \#8 SYSSIAT - 0

FIIE N20:35/10 LEN:100 - FFL - File \#13 FIFO - 10

FIIE N20:35/10 LFN:30 - FFL - File \#18 FIFCBUFFER - 1

FIIE NR0:35/10 LDN:3 - FUL - File \#3 STPRTUP - 5

|FITTER 2 DP HX

Co - File \#5 INIRLKS - 116

FIIITR 2 DP

MOTCR

IDATA CONIACT FIITER

$1, \mathrm{CF}$

$22 \mathrm{HGrtx}$ Than or $\mathrm{Eql}(\mathrm{A}>\mathrm{B})$

|| source A

11

N20:5|

25

|Source B

3291 [9:0] [22]

$1 / 1$

11 N19:11|

…

$120: 5-[18: 2]$

I

I

1120:38 - NER - File \#5 DNIRUKS - 114

MQQ - File \#12 CONIER - 1

|FUE N20:38/11 IEN:100 - FFL - File \#13 FIFO - 10

|FIIE N20:38/11 IEN:3 - COP - File \#7 MESCVIEA - 12

|N20:35/11 - -(U)- - File \#5 MVIRLS - 46, 81

FI - File \#3 STARTUP - 5

COP - File HS INIRLKS - 116

NDQ - File \#5 InRuKS - 113

File \#8 SYSSIFT - 0

|FIIE N20:35/11 LEN:100 - FFL - File \#13 FIFO - 10

|FIEE N20:35/11 LEN:30 - FFL - File \#18 FIFOBUFFER - 1

FIIS N20:35/11 IEN:3 - FLU - File \#3 STAFIUP - 5

DP - File \#5 INTRLKS - 116

FIITHR 2 DP

IDACA

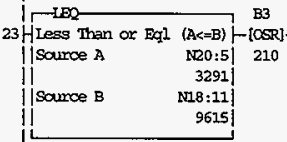

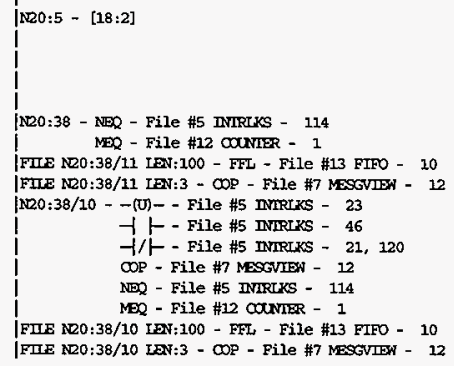

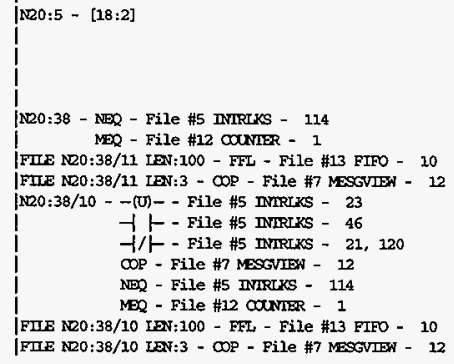

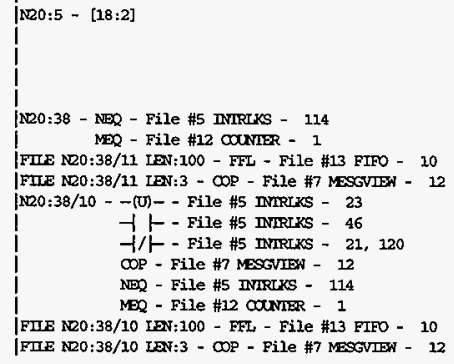

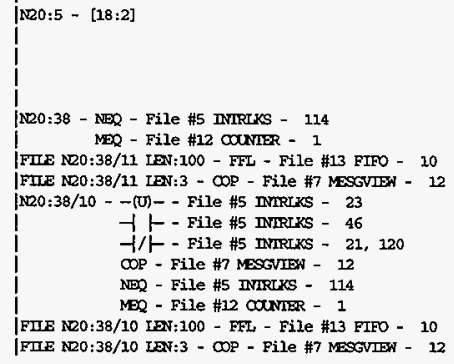

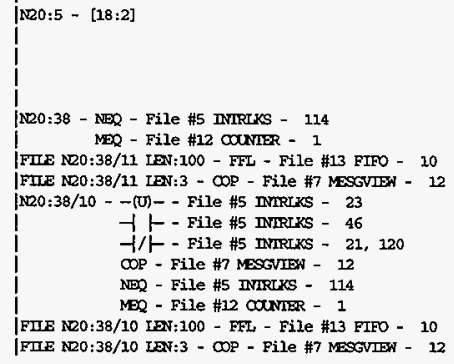

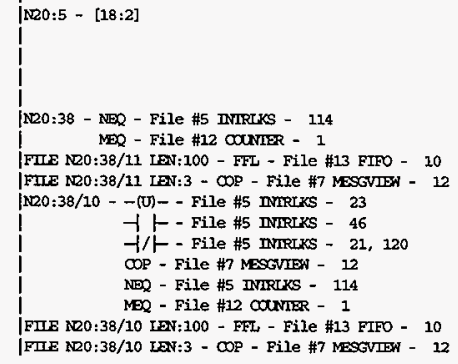

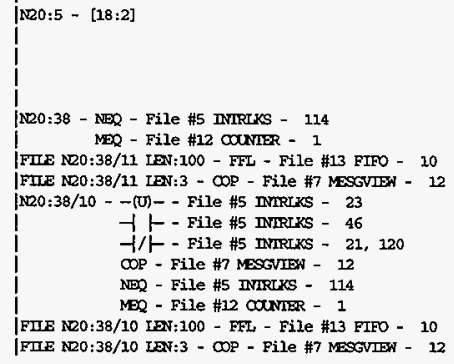

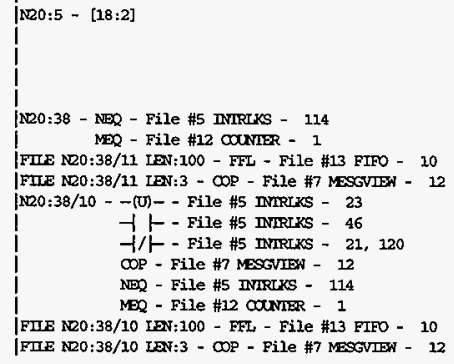

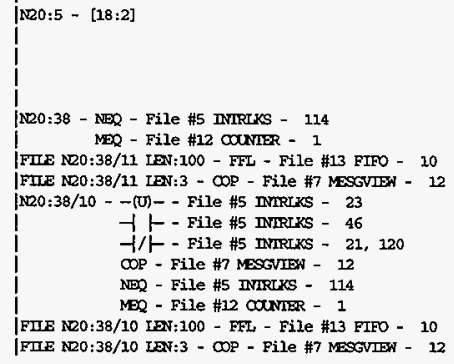

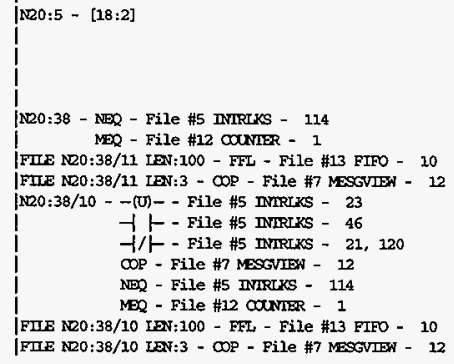

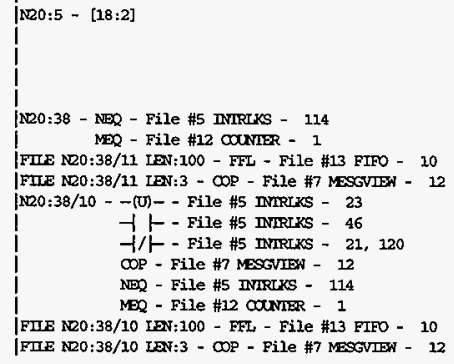

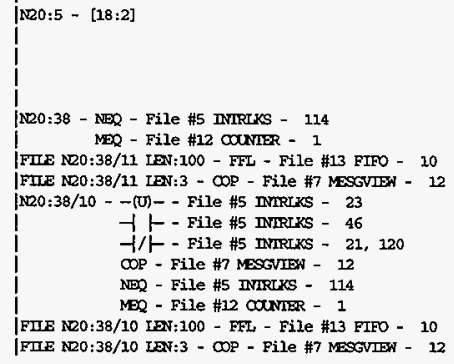




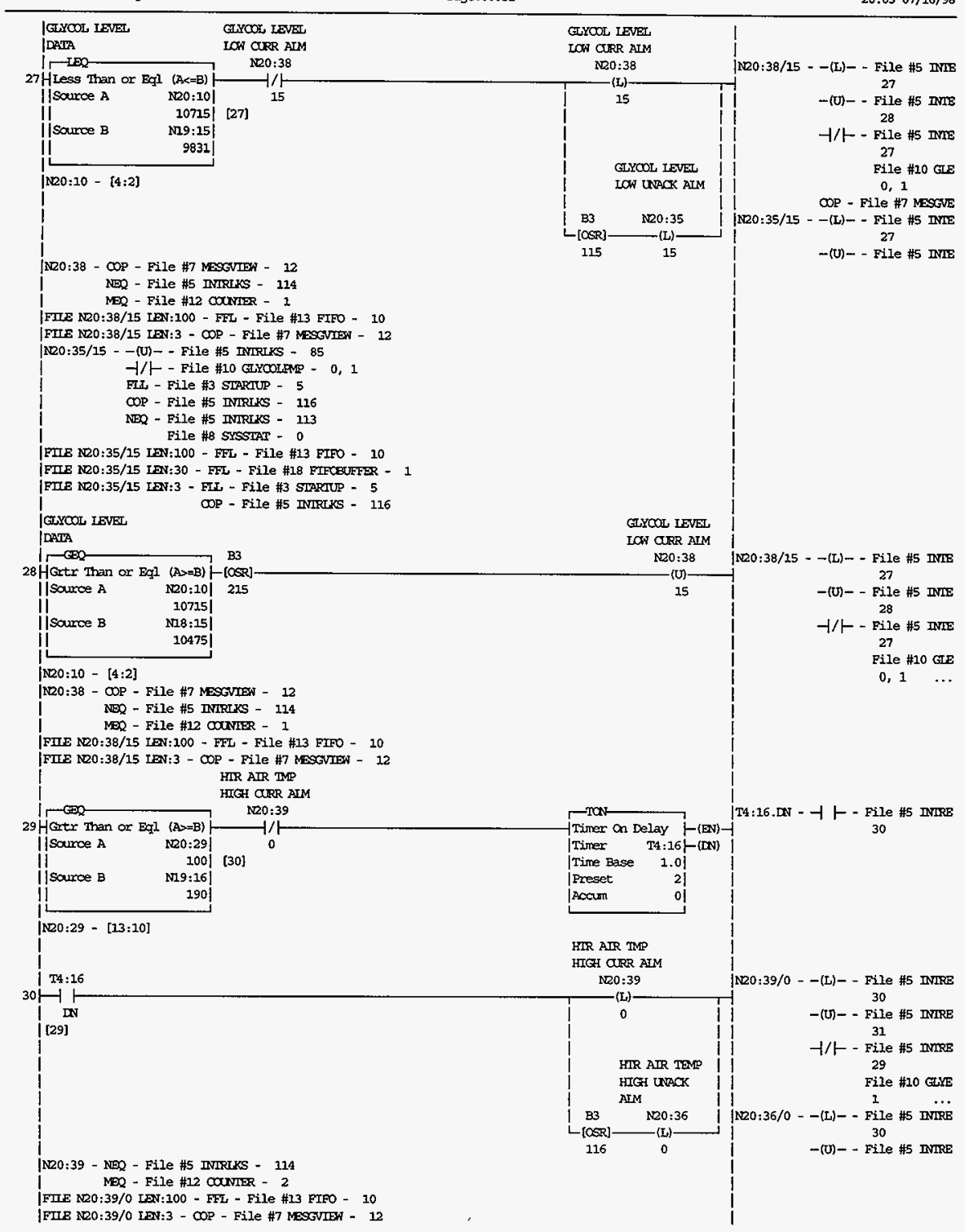


|N20:36/0 - -(U) - - File \#5 INIRIKS - 86

$\dashv / 1-$ File \#10 GHYCOLPR - 1

NEQ - File \#5 INIRUS - 113

File \#8 SYSSTAT - 0

FIIE NRO:36/0 IDN: 100 - FFL - File \#13 FIFO - 10

FLE RRO:36/0 LEN:3 - FLL - File \#3 STRRTUP - 5

COP - File \#5 INIRLS - 116

|

31 HLess than or Eql $(A<B)$ - [CSR]

|| source A N20:29| 230

II

$$
1001
$$

[source B $\quad$ N18:16

160

|N20:29 - [13:10]

N20:39 - NE2 - File \#5 INIRLKS - 114

MEO - File \#12 CONNIER - 2

FIIE NEO:39/0 IFN:100 - FFU - File H13 FIFO - 10

FIIE N20:39/0 IDN:3 - COP - File \#7 MESGIEN - 12

GTHCOL FUMP

|ON $/ \mathrm{OrF}$

[N20:39/1 - FFL - File \#13 FIFO - 10

FILE NE0:39/1 IEN:3 - COP - File \#7 MESGUIBN - 12

|R20:36/1 - -(U) - - File \#5 IMIRIKS - 87

NED - File $\# 5$ MMIRLKS - 113

File \#8 STSSTAT - 0

FTIE N20:36/1 IDN:100 - FFL - File \#13 FIFO - 10

|FIIE N20:36/1 IEN:3 - FLL - File \#3 SIARTUP - 5

COP - File \#5 INIRIKS - 116

1 -

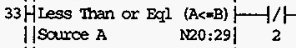

11

| Ssource B N19:18

1140

|N20:29 - [13:10]

I

I

|

|N20:39 - MEQ - File \#12 COANER - 2

FTIE N20:39/2 IFN:100 - FFL - File \#13 FIFO - 10

|FIIE N20:39/2 IEN:3 - COP - File \#7 MESGIIN - 12

|N20:36/2 - - (U) - File \#5 INIRtKS - 88

NOQ - File \#5 INIRLKS - 113

File \#8 SYSSTAT - 0

|FIE N20:36/2 IEN:100 - FFL - File \#13 FIFO - 10

|FILE ND0:36/2 IEN:3 - FLL - FIle \#3 SIARTUP - 5

COP - File \#5 INIRIKS - 116

$1, \operatorname{Gex}$

34 HGotr Than or Egl $(\mathrm{A}=\mathrm{B})$ -

||source A Ne0:29| 146

II 200

| Source B N18:18|

II $45 \mid$

NEO:29 - $\{13: 10\}$

NE0:39 - MEQ - File \#12 OONMER - 2

FIIE N20:39/2 LEN:100 - FFL - File \#13 FIFO - 10

|FIIE 100:39/2 IEN:3 - COP - File \#7 MESGVIEA - 12

HITE CAR AIM

$\mathrm{N} 20 \div 39$

|ND0:39/0 - -(L) - - File \#5 INIRE 30

-(U) - - File \#5 INIRE

31

-1/ - File \#5 IMIRE

29

File \#10 GYV

1 114

MEO - File \#12 OONIERE

|FIE N20:39/1 IEN:100 -

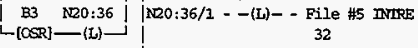

\begin{tabular}{ll|l}
119 & 1 & $-(\mathrm{U})-$
\end{tabular}
N20:39

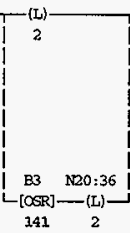

NE0:39/2 - - (L $\}-$ - File \#5 INIRE

$$
3
$$

-(U) - File \#5 INIRE

$$
39
$$

-1/1- - File \#5 INIRE 33

NDQ - File \#5 INIRLKE 114

MOO - File \#12 OaNr NDo:36/2 - -(L) - - File \#5 FIRE 33

-(U) - - File \#S INIRE 


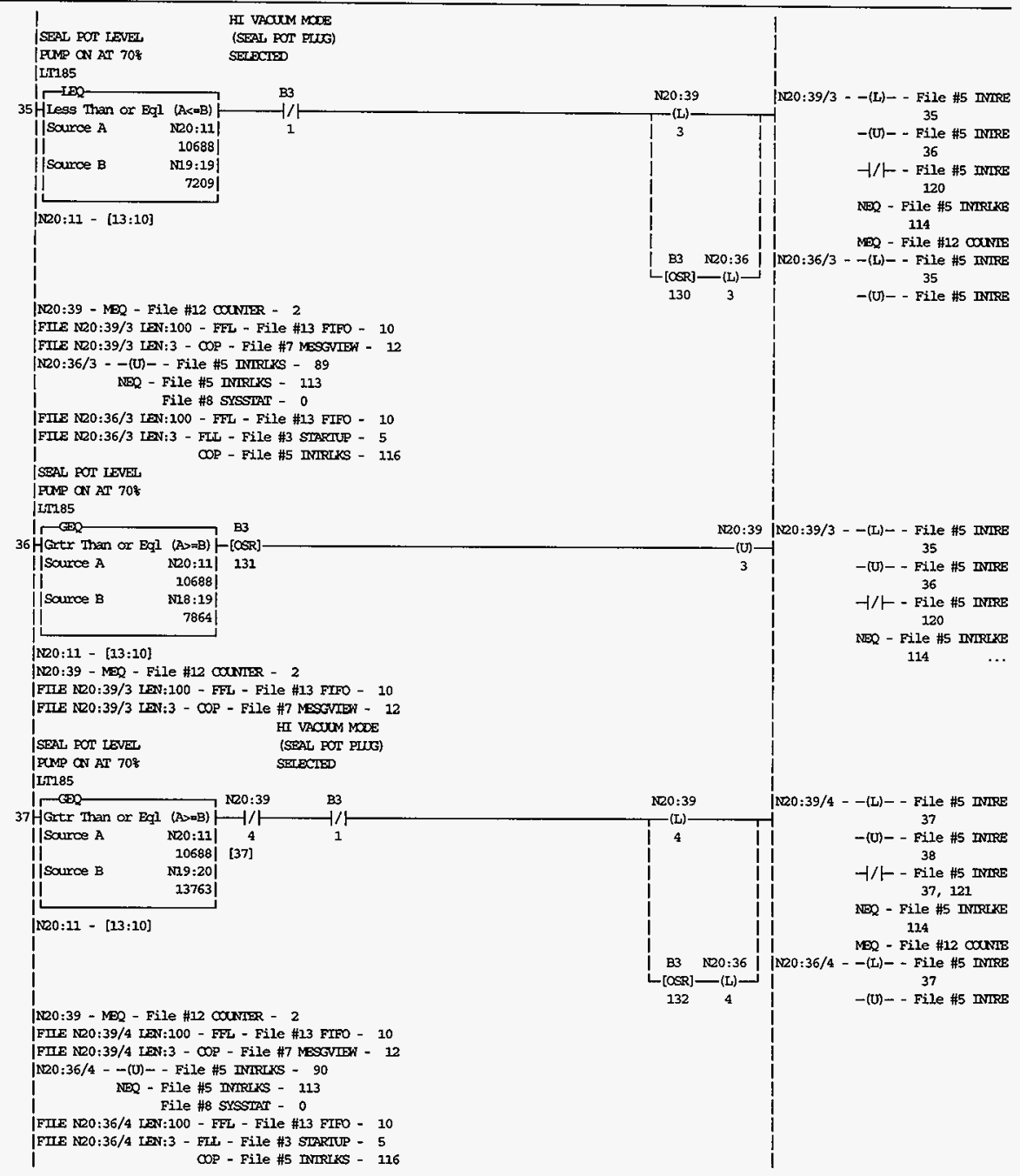




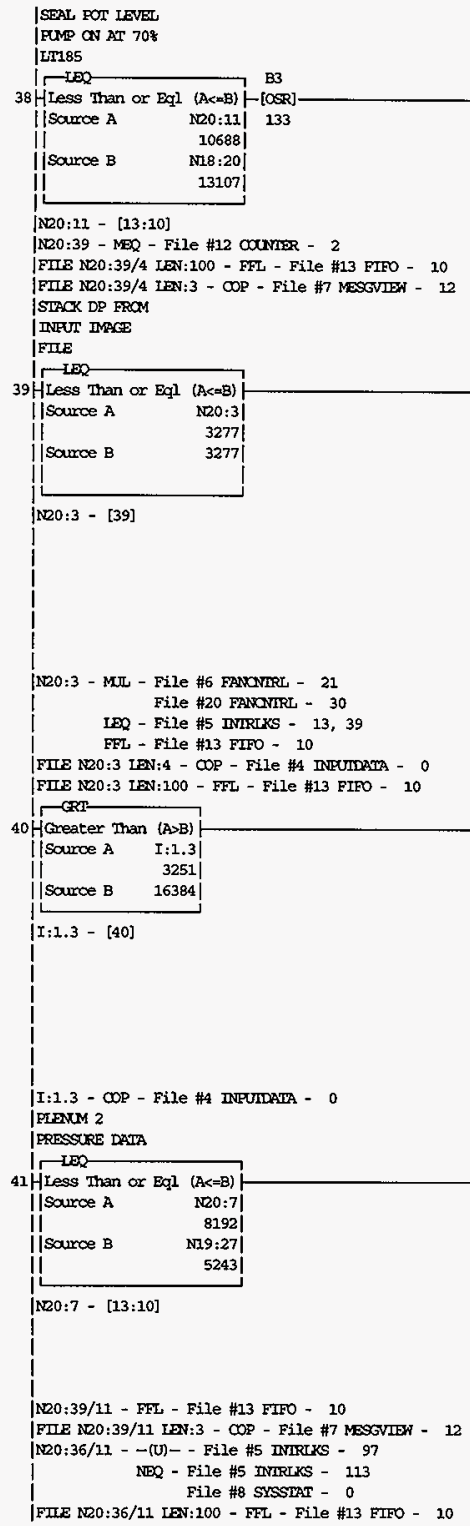$$
\left\{\begin{array}{r}
\text { IN20:3 - MU - File \#6 FANONRL - } 21 \\
\text { File \#20 FANONIR - } 30 \\
\text { IDQ - File \#5 INIRLK - 13,39 } \\
\text { FFL - File \#13 FIFO - 10 }
\end{array}\right.
$$$$
\text { I }
$$$$
\text { I }
$$$$
\text { I:1.3 - COP - File \#A INPUIDATA - } 0
$$$$
\text { i }
$$$$
\text { N20:39/11 - FFu - File \#13 FIFO - } 10
$$ 


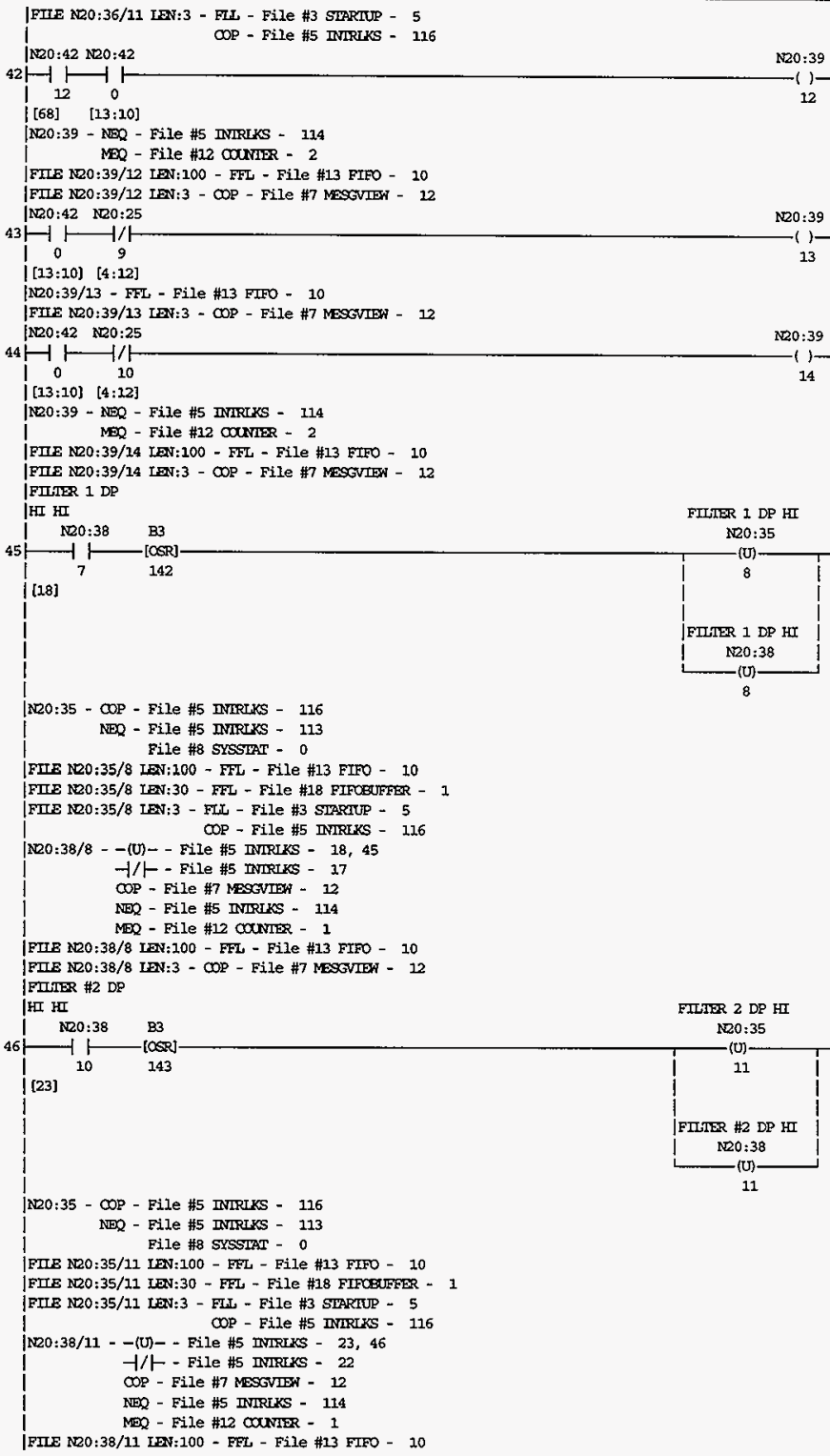




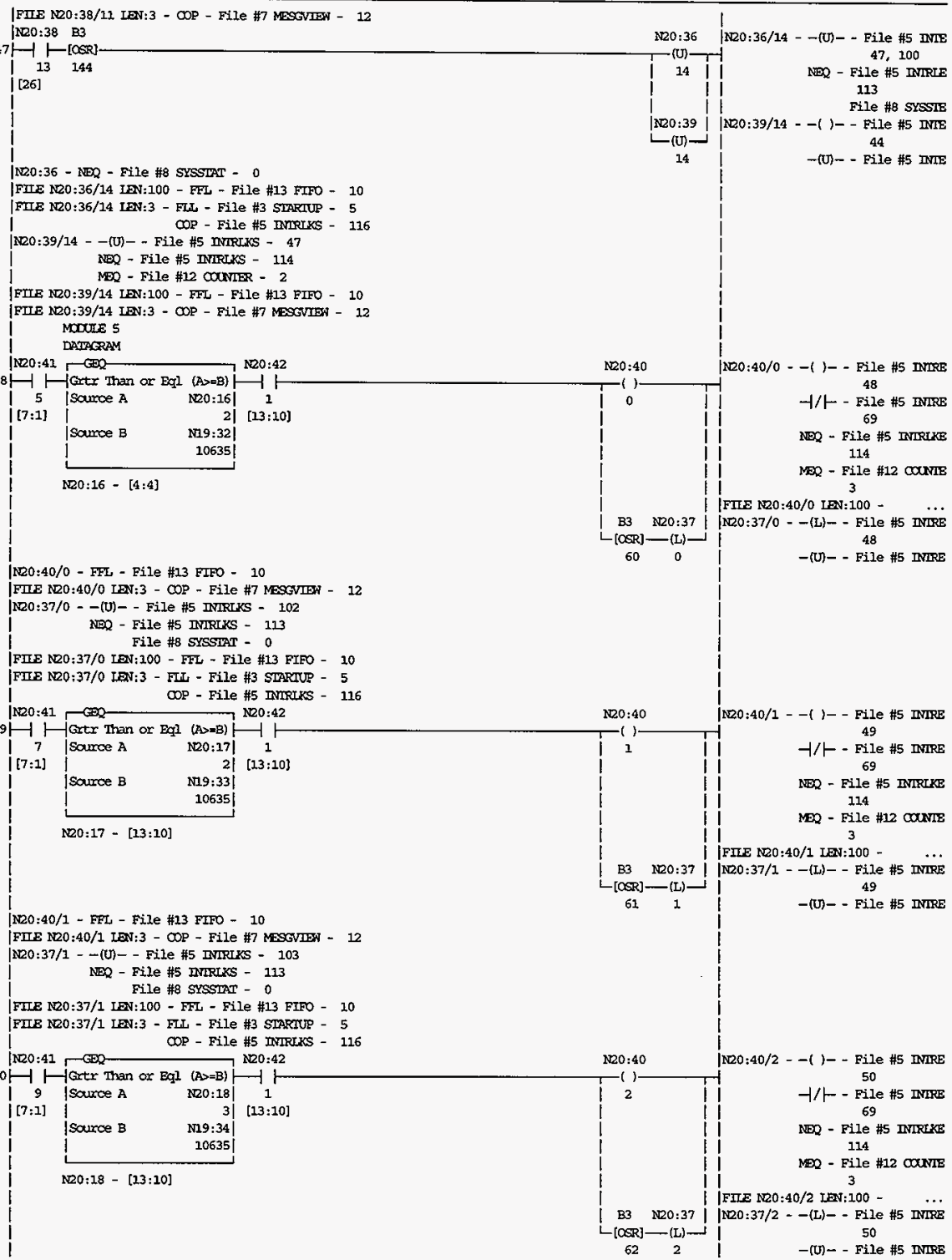


N20:40/2 - FFL - File \#13 FIFO - 10

|FIE NR0:40/2 IEN:3 - COP - File \#7 MESGVIEN - 12

N20:37/2 - - (U) - - File \#5 INIRIXS - 104

NEQ - File \#5 INIRIKS - 113

File \#8 SYSSTRT - 0

FFIE N20:37/2 IEN:100 - FFL - File \#13 FIFO - 10

FIIE N20:37/2 LEN:3 - FU, - File \#3 STARIUP - 5

COP - File \#5 INIRIKS - 116

|N20:41

N20:42

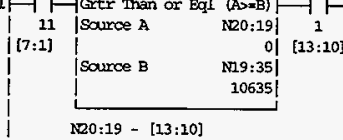

N20:40/3 - FFL - File \#13 FIFO - 10

|FIIE N20:40/3 LEN:3 - COP - File \#7 MESGVIEN - 12

|N20:37/3 - -(U) - - File \#5 IMRUKS - 105

NEQ - File \#5 IMIRINS - 113

File \#8 SYSSTAT - 0

|FIIE N20:37/3 LEN:100 - FFL - File \#13 FIFO - 10

|FIE N20:37/3 LEN:3 - FL - File \#3 SIARTUP - 5

| COP - File \#5 INIRLKS - 116

FILIER 1 ROC TMER

1 MOIOR

FAN INILKS CONTACT

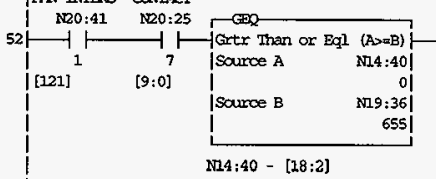

FWIER 2 ROC TIMER

MOTOR

FAN INITKS CONTACT

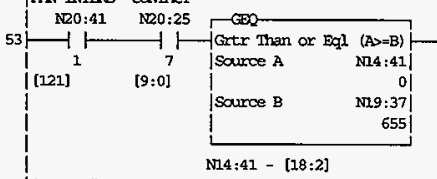

|FILTER \#1 ROC

ITMER

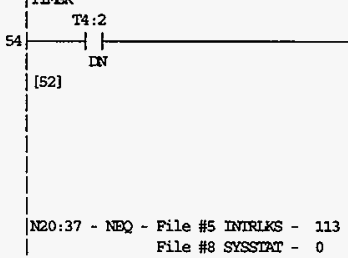

FIIE N20:37/4 LEN:100 - FFL - File \#13 FIFO - 10 FTIE N20:37/4 IEN:3 - FLI - File \#3 SIARIUP - 5

OP - File \#5 INIRLKS - 116

N20:40/4 - -(U)- - File \#5 INIRLKS - 55

| $-1 /$ - File \#5 INLEKS - 67

NEQ - File $\# 5$ INIRUKS - 114

MEQ - File \#12 COUNLER - 3

FIIE N20:40/4 IEN:100 - FFL - File \#13 FIFO - 10

|FIIE N20:40/4 IEN:3 - COP - File \#7 MESGUIEN - 12

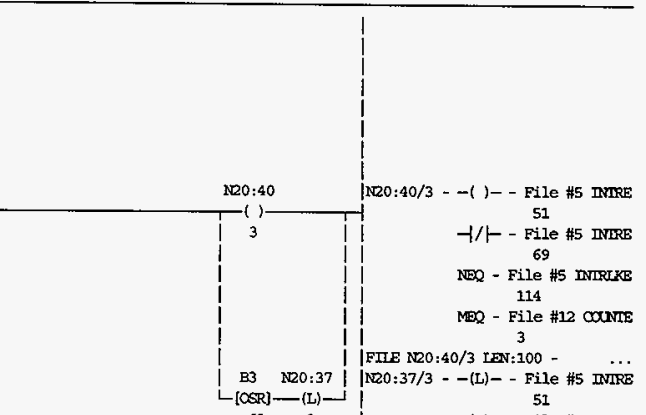

$63 \quad 3$ 


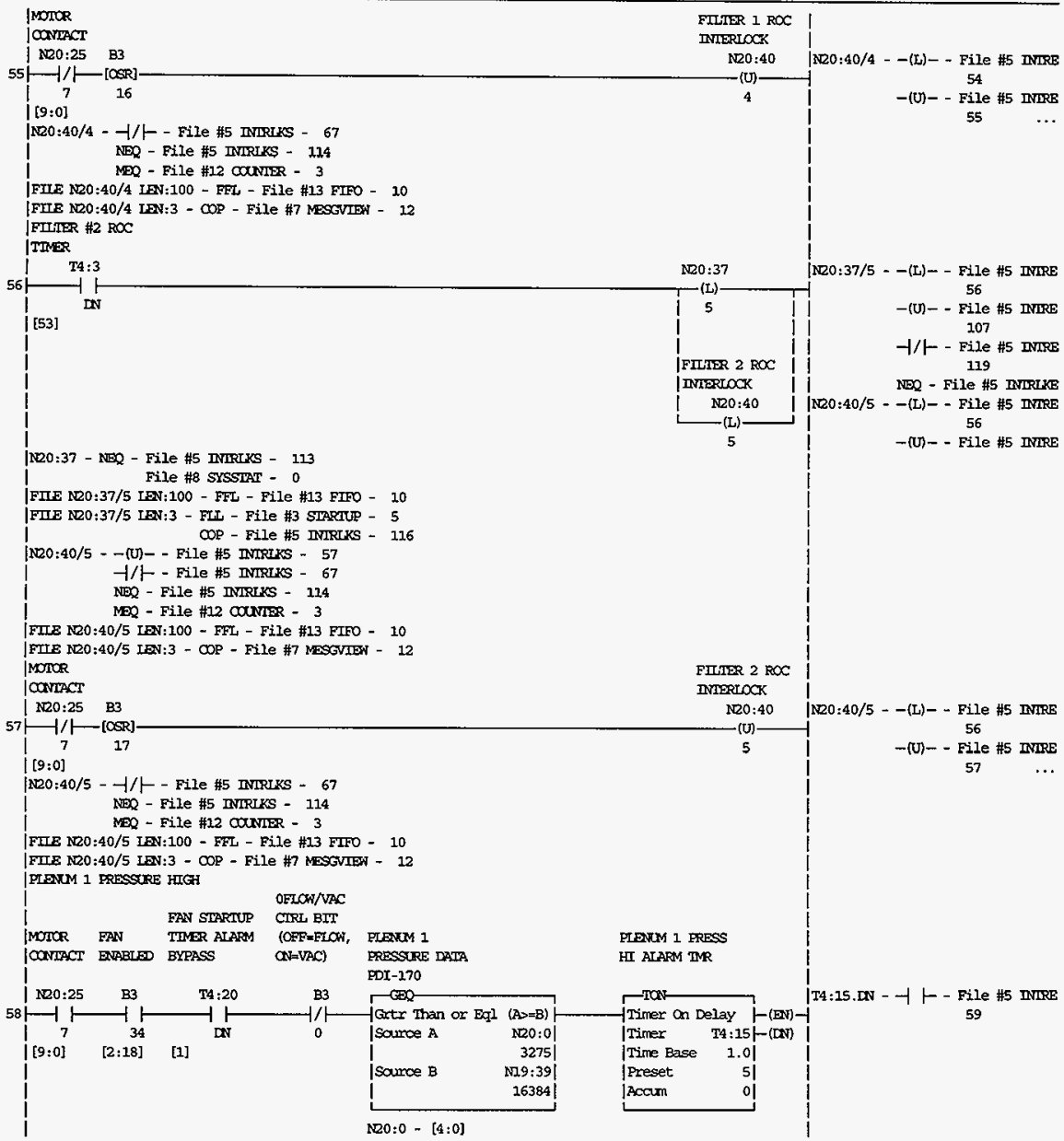




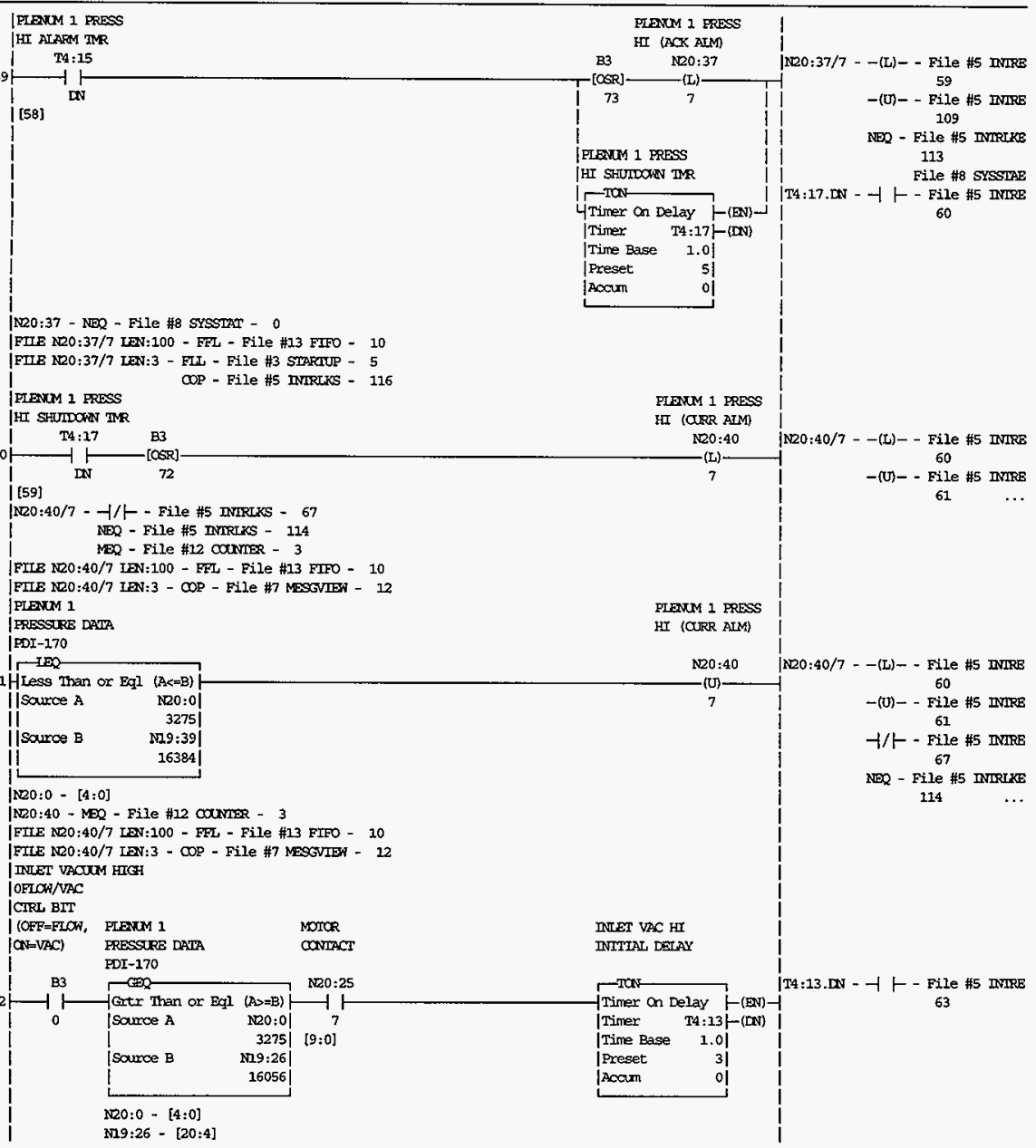




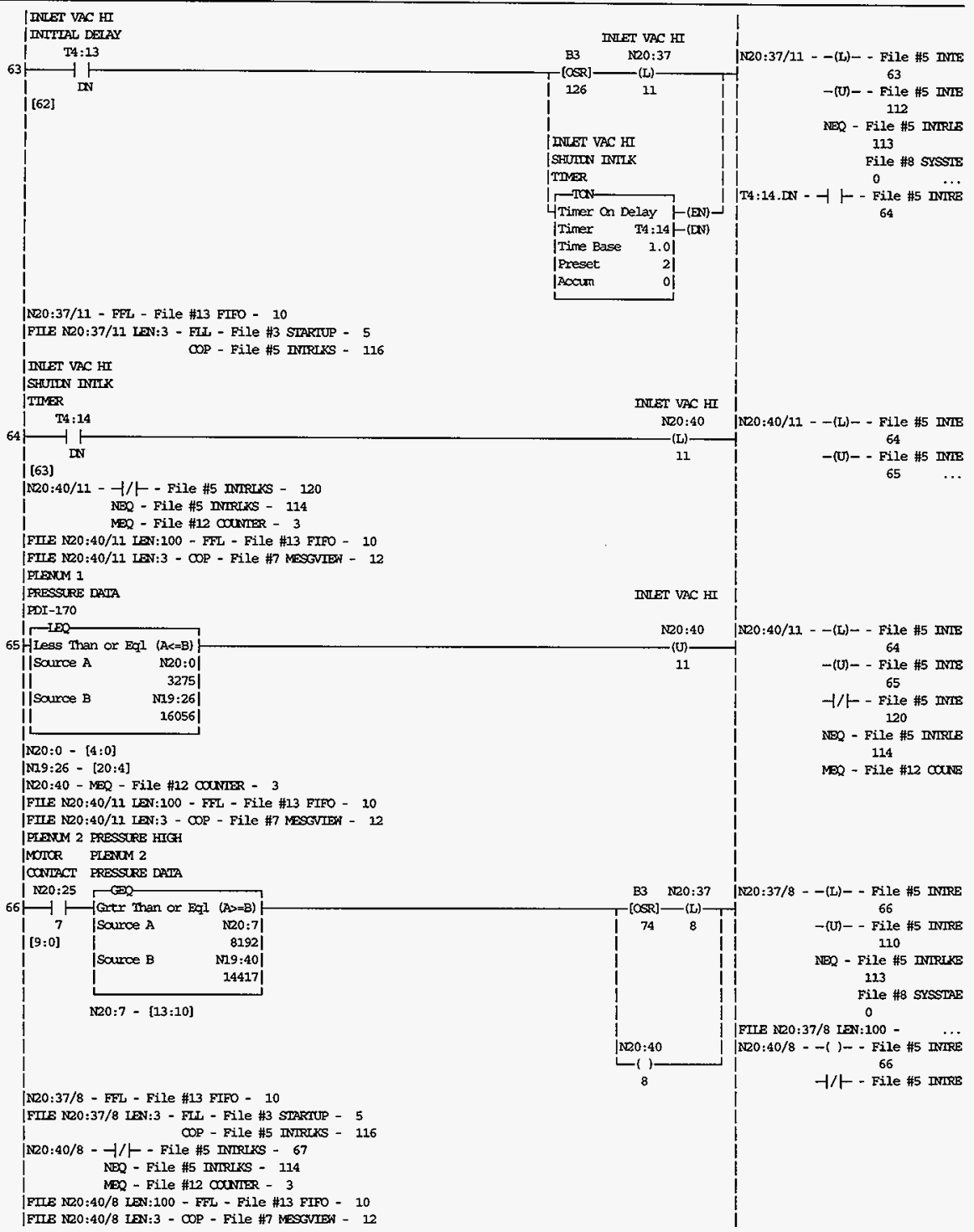




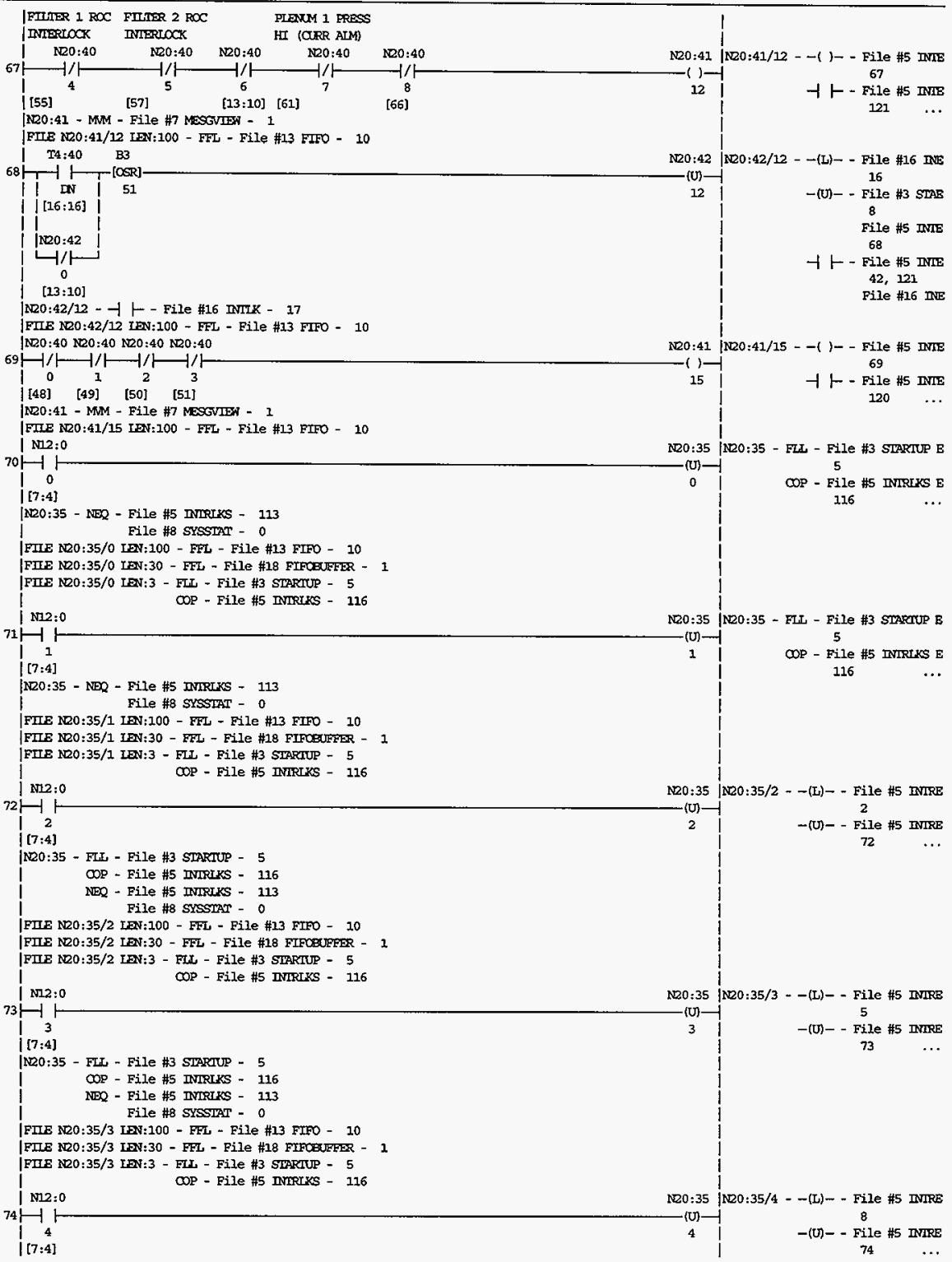




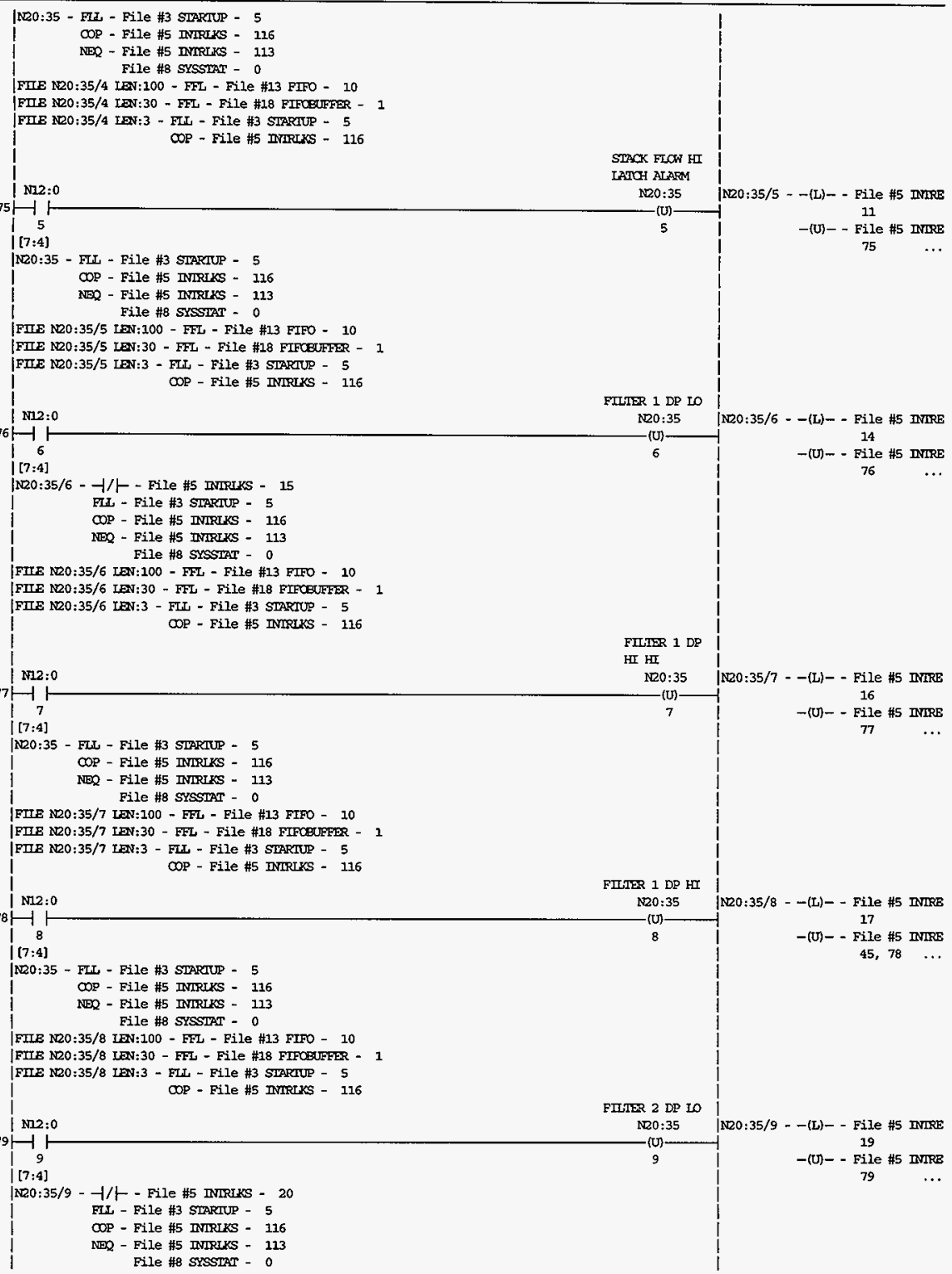


FTIE N20:35/9 IEN:100 - FFL - File \#13 FLFO - 10

|FILE N20:35/9 IEN:30 - FEL - File \#18 FIFCEUERER - I

FIIE N20:35/9 IEN:3 - FLE - File f3 STARTUP - 5

| COP - File \#5 INIRUKS - 116

I

$\mathrm{N} 2: 0$

$80 \longmapsto$

10

[ [7:4]

|N20:35 - FLL - File \#3 STARTUP - 5

COP - File \#5 INIRLKS - 116

NEQ - File \#5 INIRLKS - 113

File \#8 Srssmar - 0

|FIIS N20:35/10 EDF:100 - FFI - File \#13 FTFO - 10

|FIIE N20:35/10 IDN:30 - FFL - File \#18 FIFCEDHFER - 1

|FIIE N20:35/10 IDN:3 - FIU - File \#3 SIARIUP - 5

COP - File \#5 INIRLKS - 116

N12:0

$81 \longmapsto$

| 11

|N20:35 - FLL - File \#3 SLARIUP - 5

COP - File \#5 INIRIKS - 116

NEQ - File \#5 INIRIKS - 113

File \#8 SYSSTAT - 0

|FIIE N20:35/11 IEN:100 - FEL - File \#13 FIFO - 10

|FIIE N20:35/11 IEN:30 - FFL - File \#18 FIFOEUFERR - 1

|FILE NRO:35/11 IEN:3 - FLI - File \#3 STARTUP - 5

COP - Eile \#5 INLRLKS - 116

N12:0

$82 \longmapsto 1$

$[7: 4]$

N20:35/12 - - /

FU - File \#3 STARIUP - 5

COP - File \#5 INIRIKS - 116

NO2 - File \#5 INRIKS - 113

File \#8 SYSSTR - 0

FIIE N20:35/12 IEN:100 - FFL - File \#13 FIFO - 10

FIIE NC0:35/12 LEN:30 - FFU - File \#18 FIFCBUFFER - 1

FILE NEO:35/12 IEN:3 - FLL - File \#3 STARIUP - 5

N12:0

COP - File \#S INTRLKS - 116

$83 \longmapsto$

13

$1[7: 4]$

N20:35 - FLL - File \#3 STARIUP - 5

COP - File \#5 INIRLKS - 116

NED - File \#5 ANTRTKS - 113

File \#8 SYSSTAT - 0

FIIE NDO:35/13 IEN:100 - FFL - File \#13 FTFO - 10

|FTIE N20:35/13 IEN:30 - FFL - File \#18 FIFCEUFFER - 1

FILE N20:35/13 IEN:3 - FLI - File \#3 SIARTUP - 5

N2:0

COP - File \#5 INTRLSS - 116

84

84.

[ $77: 4]$

KO20:35 - NE2 - File \#5 INIRLKS - 113

File \#8 SYsSTAT - 0

|FIIE N20:35/14 IDN:100 - FFL - File \#13 FIFO - 10

FIIE N20:35/14 IEN:30 - FFL - File \#18 FIFOBUFFER - 1

[FIIE N20:35/14 LEN:3 - FLU - File \#3 SIARTUP - 5

COP - File \#5 INIRLKS - 116

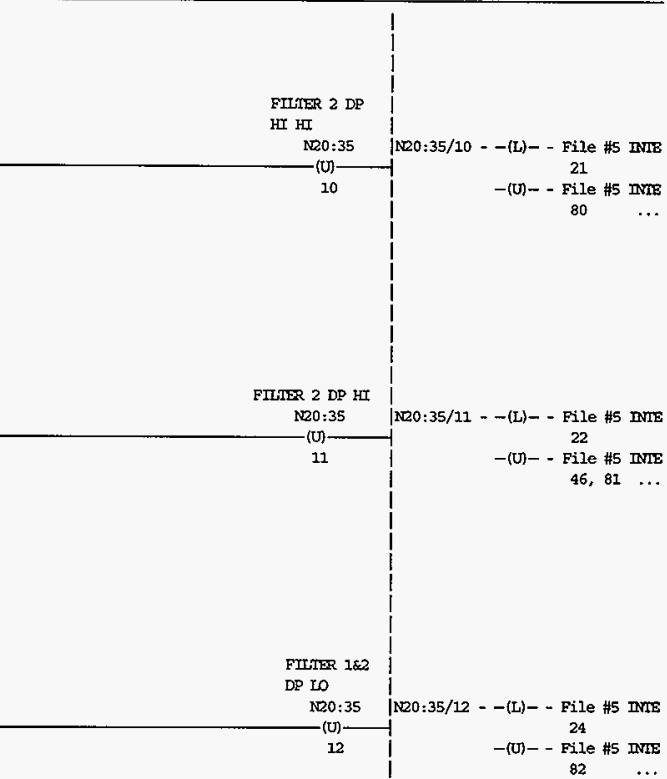

HNF-2934

Rev 0

A-33 


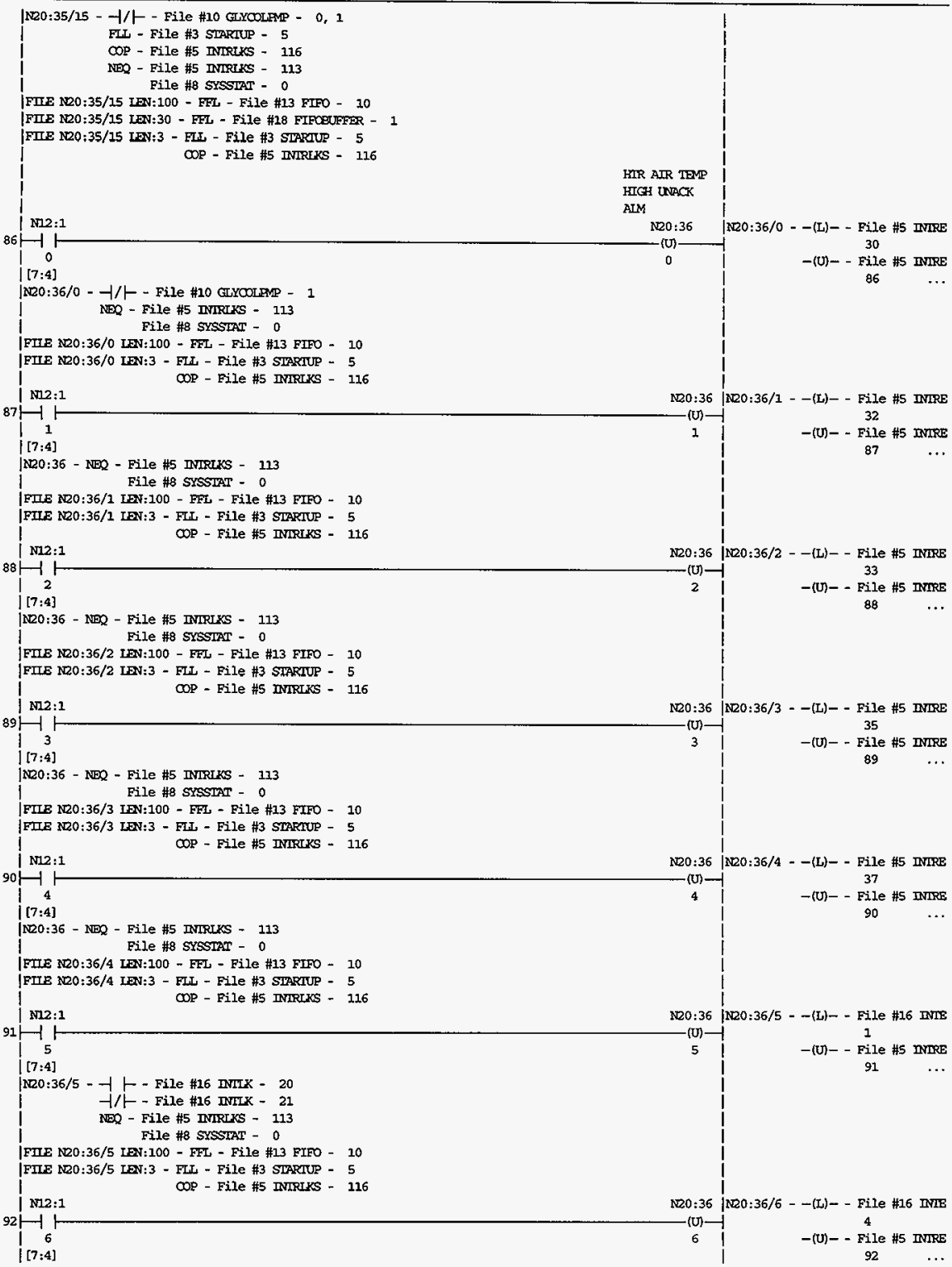


[N20:36 - NEO - File \#5 INIRLS - 113

File \#8 SYSSTPT - 0

|FIE N20:36/6 IEN:100 - FEL - File \#13 FIFO - 10

FIIE N20:36/6 IEN:3 - FLL - File \#3 STRRIUP - 5

N12:1

COP - File \#5 RMIRIKS - 116

93

17

[ [7:4]

IN20:36/7 - -

- $/$ - File \#16 IMTLK - 21

NEQ - File \#5 INIRIKS - 113

File \#8 SYSSTAT - 0

FIIE N20:36/7 LAN: 100 - FFL - File \#13 FIFO - 10

FTIE NDO:36/7 LEN:3 - FLC - File \#3 SIARIUP - 5

N12:1

COP - File \#5 MMIRLIS - 116

941

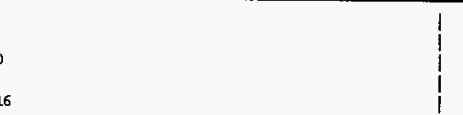

HNF-2934

Rev 0 


\begin{tabular}{|c|c|c|c|c|}
\hline \multirow{4}{*}{101} & \multicolumn{2}{|c|}{ COP - File \#5 INIRLKS - 116} & \multirow{3}{*}{$\begin{array}{c}\mathrm{NDO}: 36 \\
-(\mathrm{U})\end{array}$} & \multirow{3}{*}{ N20:36 - NEQ - File \#5 DNIRIKS E } \\
\hline & ( & & & \\
\hline & -1 & & & \\
\hline & 15 & & 15 & File \#8 SXSSTAT E \\
\hline & {$[7: 4]$} & & & $0 \quad \ldots$ \\
\hline & N20:36/15 - FFL - File \#13 FIFO - 10 & & & 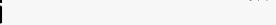 \\
\hline & FIIE N20:36/15 LEN:3 - FU - File \#3 SIARTUP & -5 & & 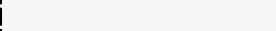 \\
\hline & COP - File \#5 IMIRKS & -116 & & \\
\hline & N N12:2 & & $\mathrm{N} 20: 37$ & N20 $237 / 0$ - - (L) - - File \#5 TMIRB \\
\hline 102 & 1 & & $(\mathrm{U})$ & 48 \\
\hline & 0 & & 0 & -(U) - - File \#5 INIRE \\
\hline & $\mid(7: 4)$ & & & $102 \quad \ldots$ \\
\hline & |NE0:37 - NEO - File \#5 INIRLKS - 113 & & & 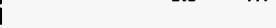 \\
\hline & File \#8 SYSSTAT - 0 & & & | \\
\hline & FIIE N20:37/0 IEN:100 - EFL - File \#13 FIFO - & 10 & & 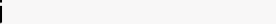 \\
\hline & FUIE NLO:37/0 IEN:3 - FLU - File \#3 STARTIS - & 5 & & i \\
\hline & COP - File \#5 INIRHAS - & 116 & & \\
\hline & $2 \times 12: 2$ & & $20: 37$ & NCO0:37/1 - -(L) - - File \#5 IMRE \\
\hline 2031 & 1 & & $-(t)-$ & 49 \\
\hline & 1 & & 1 & $-(\mathrm{U})-$ - File $\# 5$ INIRE \\
\hline & {$[7: 4]$} & & & $103 \quad \ldots$ \\
\hline & N20:37 - NEQ - File \#5 INIRIKS - 113 & & & . \\
\hline & File \#8 SYSSTPI - 0 & & & 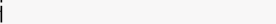 \\
\hline & |FIIE L20:37/1 LEN:100 - FFL - File \#13 FIFO - & 10 & & 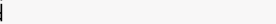 \\
\hline & FIIS N20:37/1 LEN:3 - FLL - File \#3 STARTUP - & 5 & & 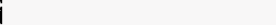 \\
\hline & COP - File H5 INRTKS - & 116 & & \\
\hline & $\mathrm{N} 12: 2$ & & $\mathrm{~N} 20: 37$ & |N20:37/2 - -(L) - - File \#5 InIRE \\
\hline 104 & -11 & & $\longrightarrow(\mathrm{U})-$ & 50 \\
\hline & 2 & & 2 & -(U) - - File \#5 INIRE \\
\hline & {$[7: 4]$} & & & $204 \quad \ldots$ \\
\hline & N20:37 - NE2 - File \#5 INIRLKS - 113 & & & 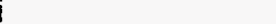 \\
\hline & File \#8 SISSTAT - 0 & & & 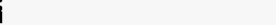 \\
\hline & FIIE NE0:37/2 LEN: 100 - FFL - File \#13 FIFO - & 10 & & 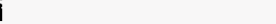 \\
\hline & FHE NE0:37/2 LEN:3 - FLU - File \#3 SIARTUP - & 5 & & i \\
\hline & COP - File \#5 INIRLKS - & 116 & & i \\
\hline & $\mathrm{M} 12: 2$ & & $120: 37$ & IN20:37/3 - -(L)- - File \#S IMRR \\
\hline $105 \vdash$ & 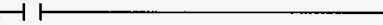 & & $-(\mathrm{U})-$ & 51 \\
\hline & 3 & & 3 & -(U)- - File \#5 INIRE \\
\hline & {$[7: 4]$} & & & $105 \quad \ldots$ \\
\hline & N20:37 - NDQ - File \#5 INIRIKS - 113 & & & i \\
\hline & File \#8 SYSSTAT - 0 & & & 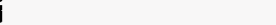 \\
\hline & |FItE DD0:37/3 LDN:100 - FFL - File \#13 FIFO - & 10 & & 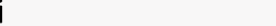 \\
\hline & FILE N20:37/3 LEN:3 - FLI - File \#3 SIARIUP - & 5 & & i \\
\hline & COP - File \#5 INIRLKS - & 116 & & \\
\hline & N12:2 & & NR0:37 & |N20:37/4 - -(L)- - File \#5 INIRB \\
\hline 106 & 11 & & $-(\mathrm{U})-$ & 54 \\
\hline & 4 & & 4 & -(U) - - File \#5 INRB \\
\hline & {$[7: 4\}$} & & & $106 \quad \ldots$ \\
\hline & N20:37/4 - - //F - File \#5 INIRLKS - 119 & & & I \\
\hline & NEQ - File \#5 INIRKS - 113 & & & i \\
\hline & File \#8 SYSSIFT - 0 & & & i \\
\hline & FDE NR0:37/4 LEN: 100 - FFL - File \#13 FIFO - & 10 & & 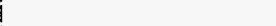 \\
\hline & FIIE NLO:37/4 LEN:3 - FLU - File \#3 SIARIUP - & 5 & & 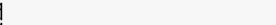 \\
\hline & COP - File \#5 MMRUKS - & 116 & & \\
\hline & N12:2 & & $\mathrm{N} 20: 37$ & N20:37/5 - -(L)- - File \#5 nNIRE \\
\hline 1071 & $\mapsto \longmapsto$ & & $-(\mathrm{U})-$ & 56 \\
\hline & 5 & & 5 & -(U)- - File \#S INLRE \\
\hline & {$[7: 4]$} & & & $107 \quad \ldots$ \\
\hline & NDO:37/5 - - / & & & i \\
\hline & NEQ - File \#5 INIRLKS - 113 & & & 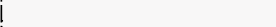 \\
\hline & File \#8 SYSSTRT - 0 & & & 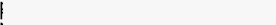 \\
\hline & |FTE RR0:37/5 LEN: 100 - FFL - File \#13 FIFO - & 10 & & j \\
\hline & |FTE N20:37/5 IEN:3 - FU - File \#3 STARTUP - & 5 & & j \\
\hline & COP - File \#5 IMIRKS - & 116 & & \\
\hline & N12:2 & & N20:37 & IN20:37 - NDO - File \#5 INIRUKS E \\
\hline $108 \vdash$ & $\mapsto 1$ & & $(\mathrm{U})-$ & 113 \\
\hline & 6 & & 6 & File \#8 SXSSTAT E \\
\hline & {$[7: 4]$} & & & $0 \quad \ldots$ \\
\hline & N20:37/6 - FFL - File \#13 FIFO - 10 & & & 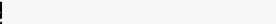 \\
\hline & FTLE N20:37/6 IEN:3 - FU, - File \#3 SIARTIU - & 5 & & 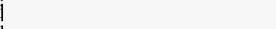 \\
\hline 1 & COP - File H5 INIRLK . & 116 & & i \\
\hline
\end{tabular}




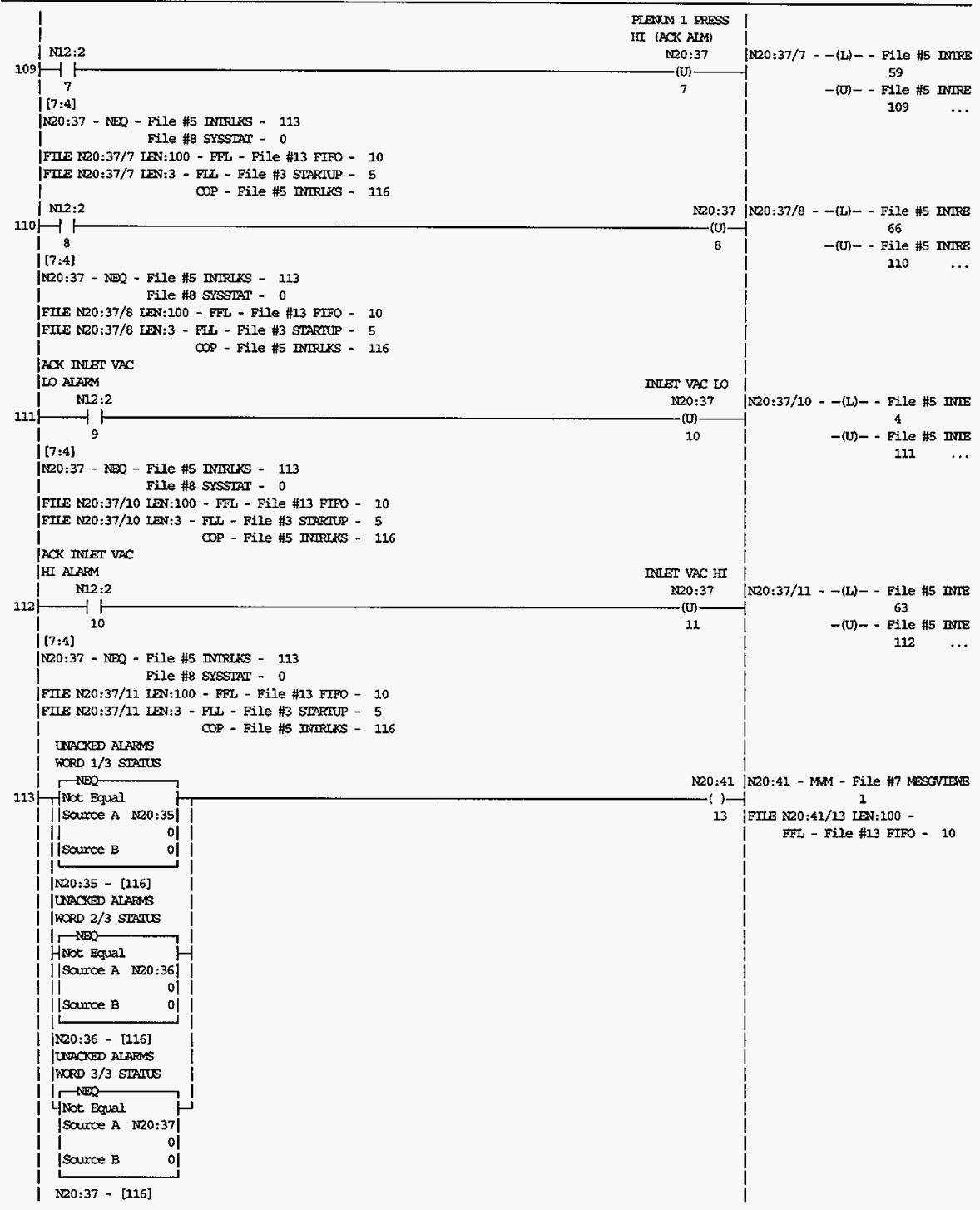




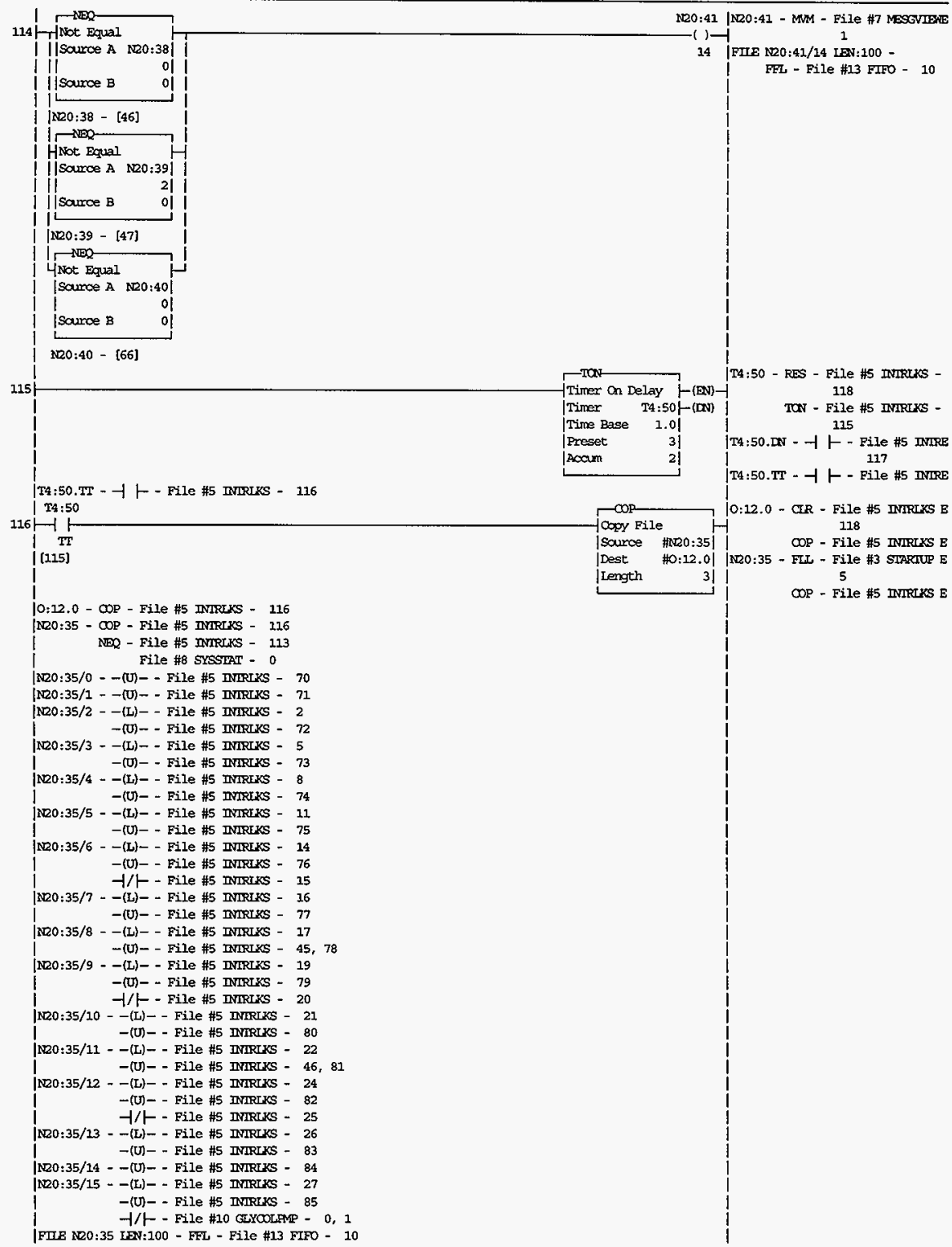




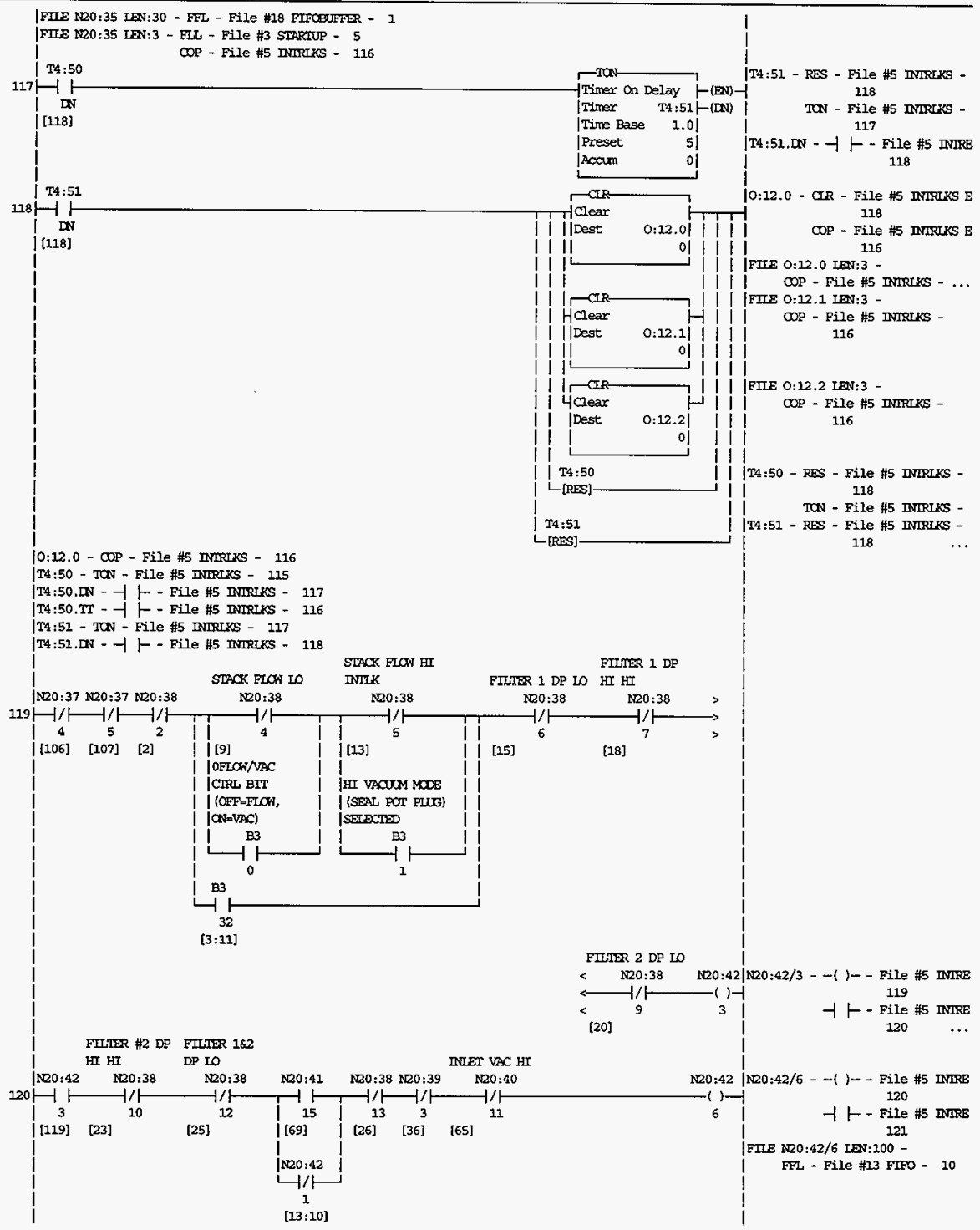




[OW INFUT INIKK




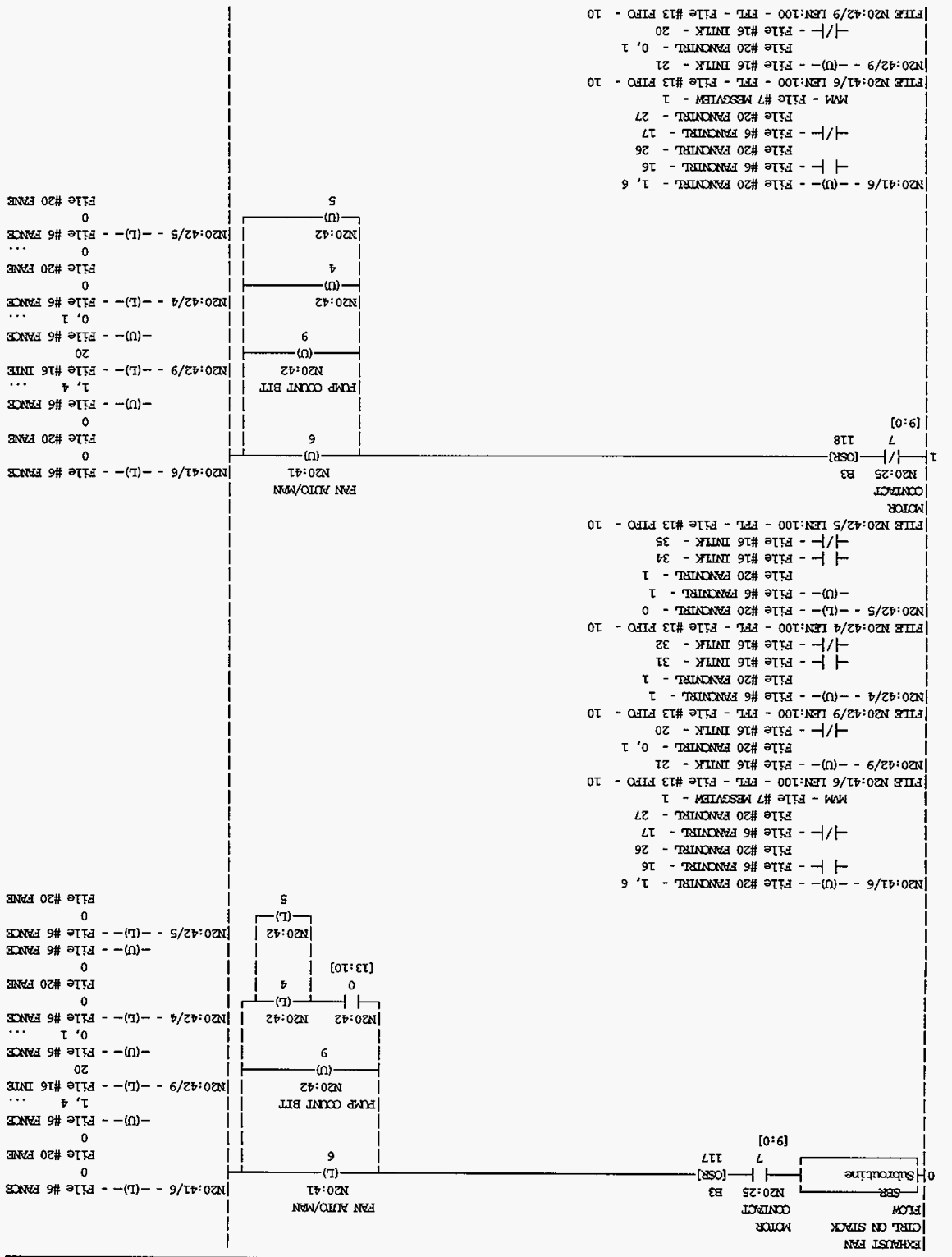




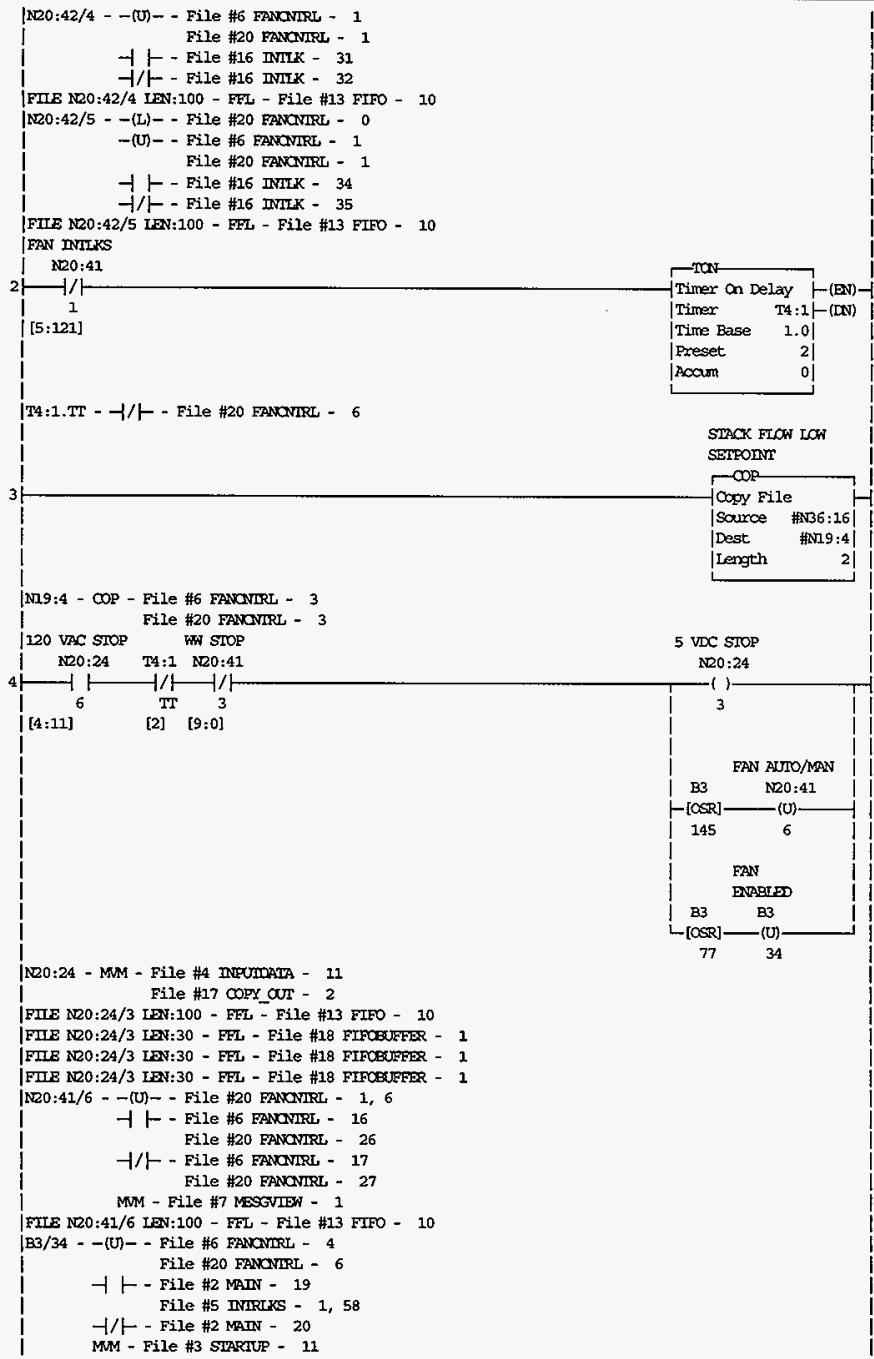$$
\text { i }
$$ 


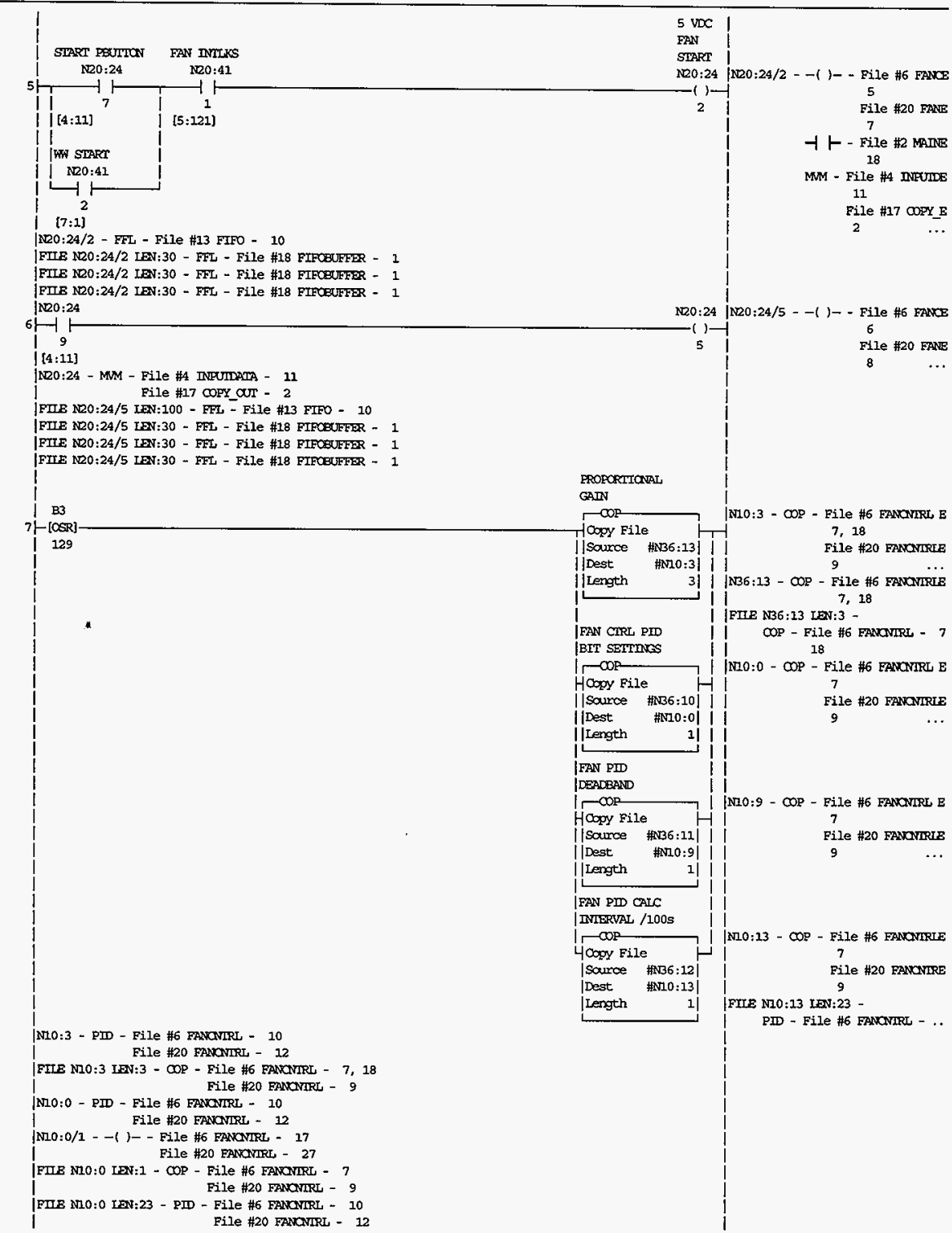




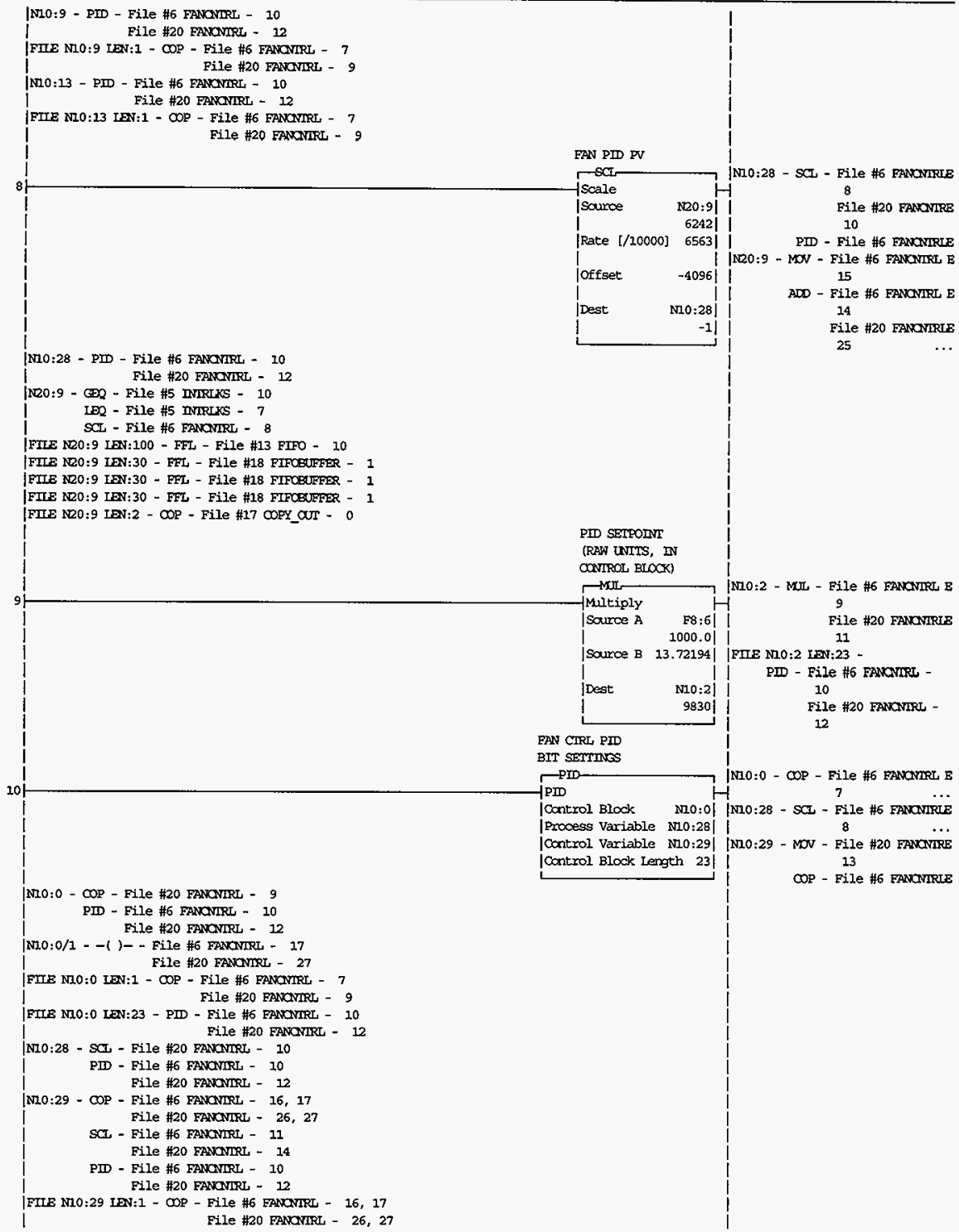




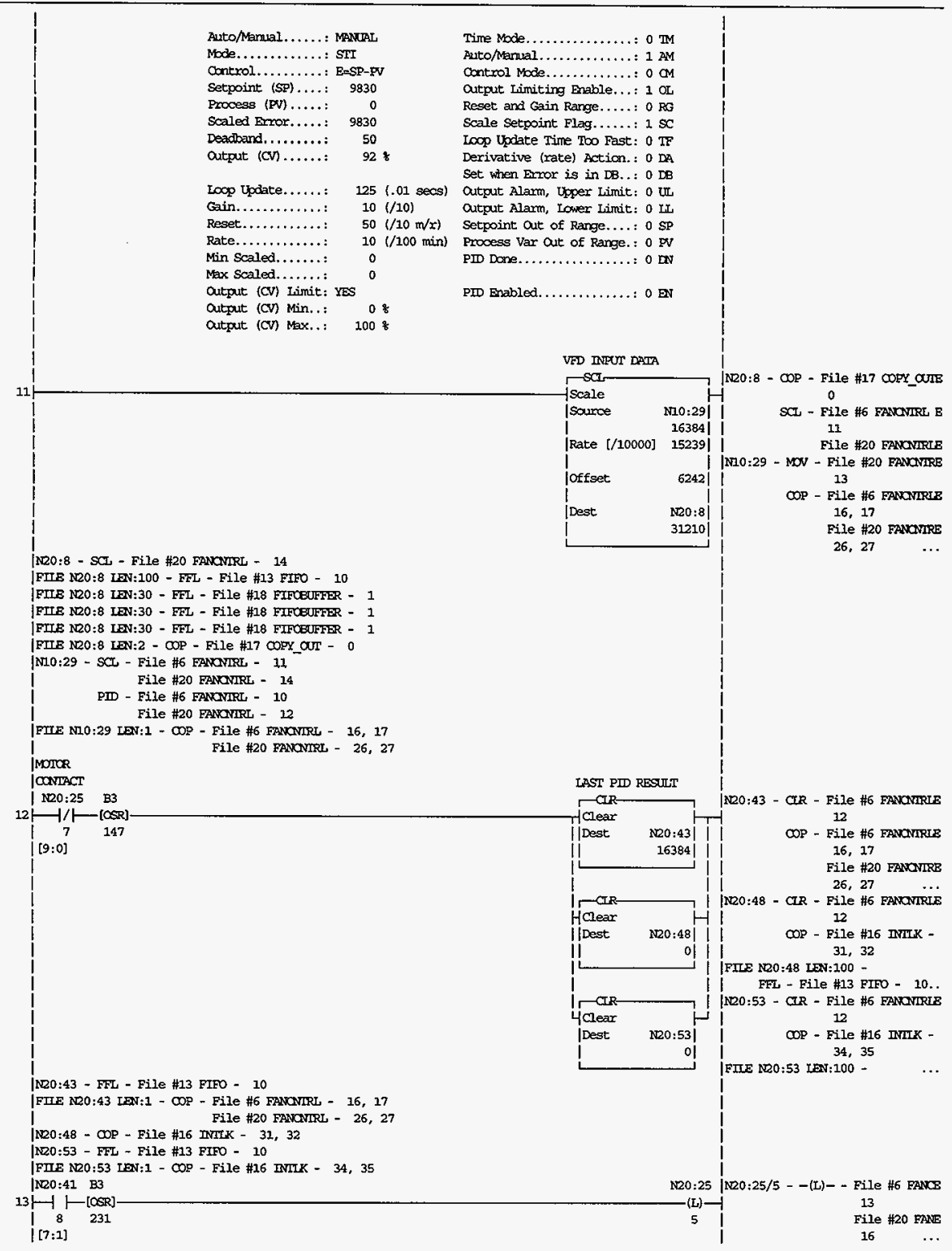


Fortable 1000 CFM Exhauster Program

|N20:25/5 - - (U) - - File \#3 STARIUP - 7

MM - File \#4 INFUIDATA - 12

File \#17 COFY_or - 3

|FIIE RDO:25/5 IRN:100 - FFU - File \#13 FIFO - 10

|FTEE RR0:25/5 LON:30 - FFL - File \#18 FIFOBUFFER - 1

|FTE ID20:25/5 IEN:30 - FEL - File \#18 FIFCEUFFER - I

FIIE NR0:25/5 IEN:30 - FFL - File \#18 FIFCEUFFER - 1

HNF-2934

Rev 0

A-46 


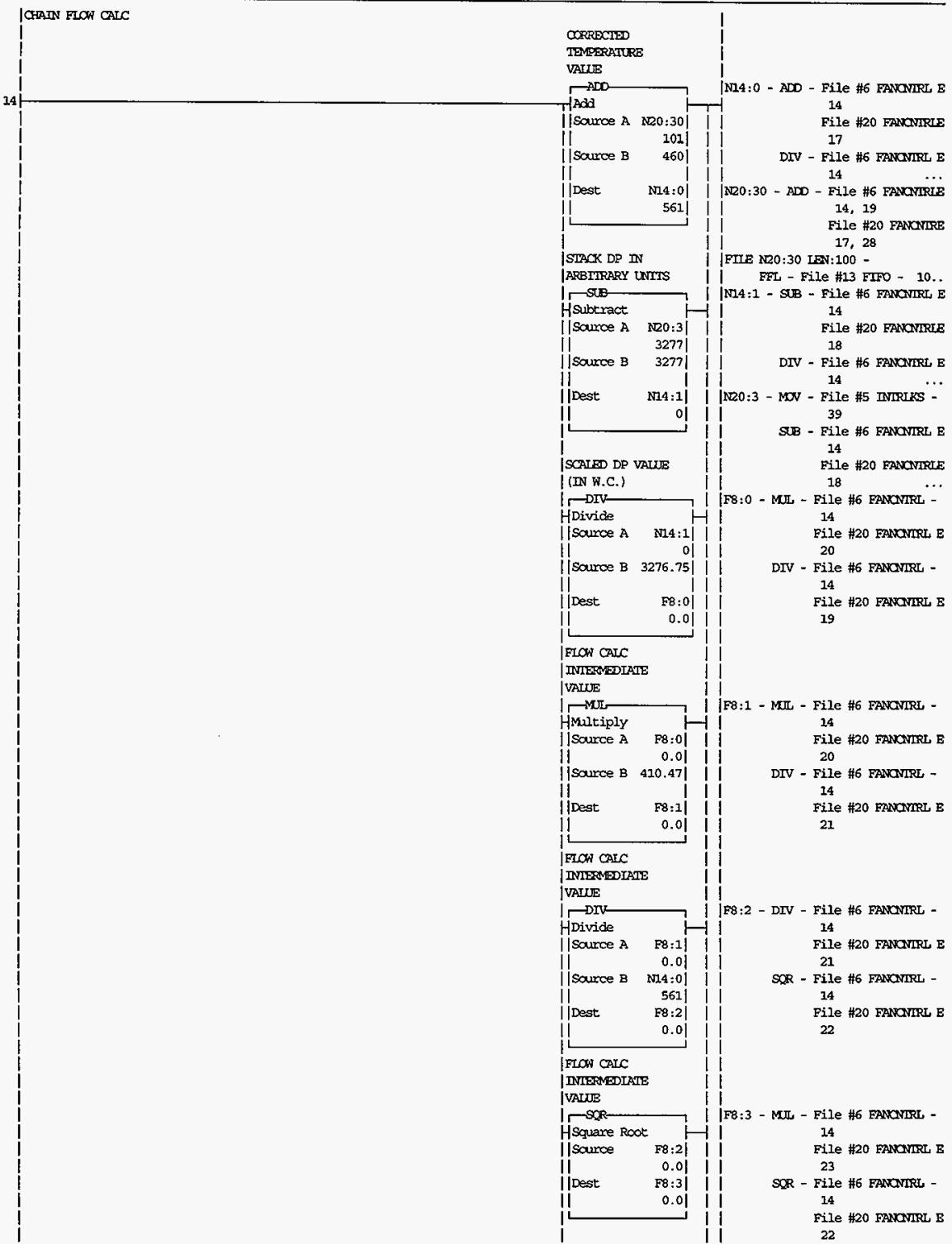



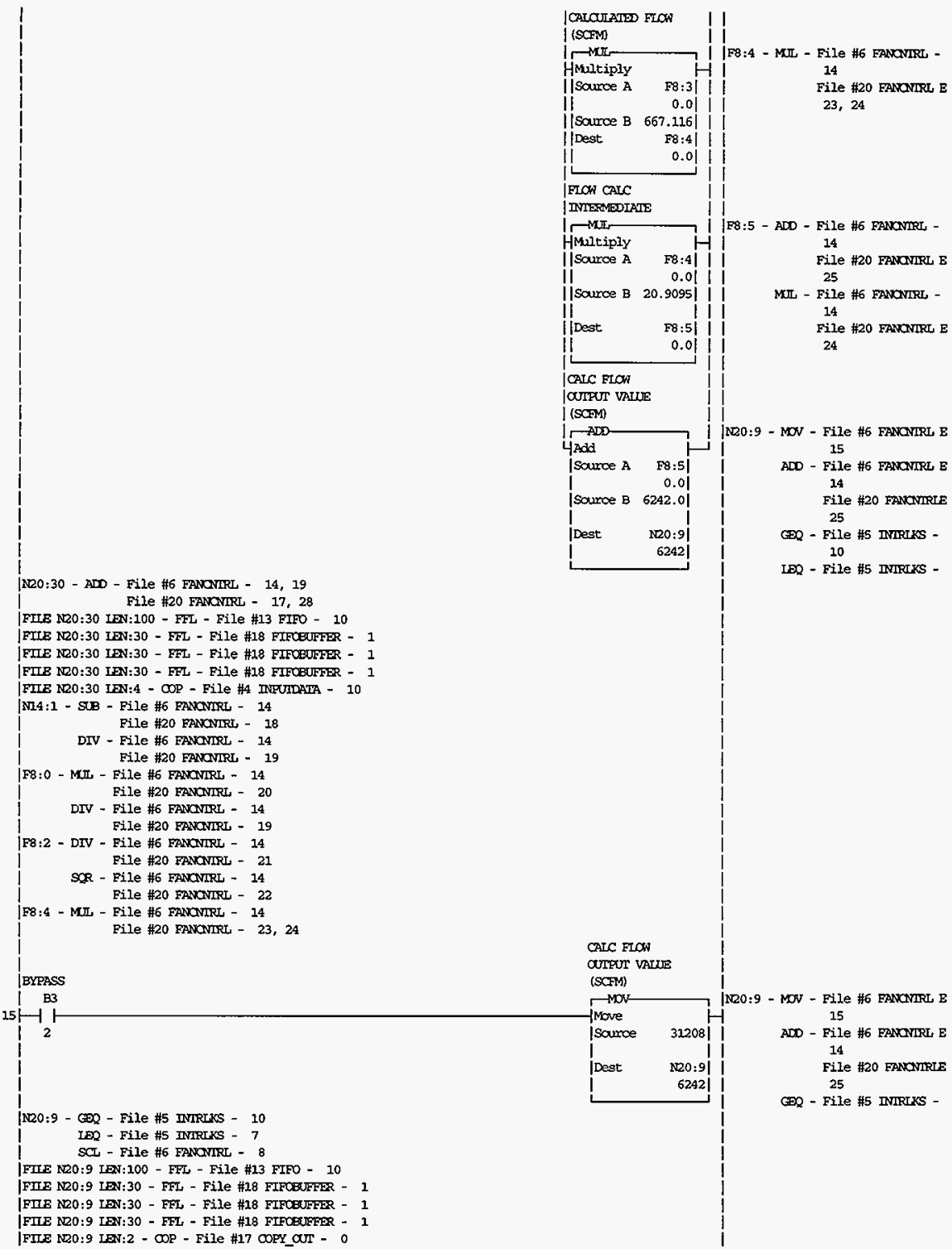

FIIE N20:30 IEN:100 - FFL - File \#13 FIFO - 10 |FIIE NDO:30 IEN:30 - ERL - File \#18 FIFCEUFFER - 1 |FILE NDO:30 LEN:30 - FFL - File \#18 FIFOEUFFER - 1 |FIB NLO:30 IEN:30 - FFL - File \#18 FIFOBUFFER - 1 FIIE NQ0:30 IEN:4 - COP - File \#4 INEUIDATA - 10

STB - File \#6 EANavirt File \#6 EPNONTRL - 14 File \#6 FANaNIRL - 14 File \#20 FANCNIRL - 22 armor vaLU: (SCFM)

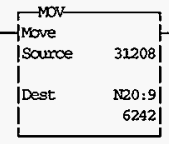

|NR0:9 - MON - File \#6 EANONIRL E 15 ADD - File \#6 FANONIRL E 14 File \#20 FANOMIRE 25 GD - File \#5 INIRUS - 


\begin{tabular}{|c|c|c|c|}
\hline & |EAN ALIO/MAN & IAST PID RESTIT & \\
\hline & $N 20: 41$ & $\Gamma_{-\infty}$ & iN20:43 - CRR - File \#6 FANONIRLE \\
\hline 161 & 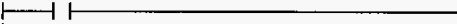 & foopy File & 12 \\
\hline & 6 & [Source \#N10:29| & op - File \#6 EPNONIPLE \\
\hline & {$[4]$} & |Dest \#N20:431 & |N10:29 - MOV - File \$20 FANONIRE \\
\hline & & |lergth I] & 13 \\
\hline & & & COP - File \#6 PANONIRLE \\
\hline & ¡R20:43 - COP - File \#6 FANOrIR - 16, 17 & & 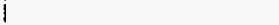 \\
\hline & | File \#20 EANONIRL - 26,27 & & i \\
\hline & FIIE N20:43 LEN:100 - FFL - File \#13 FIFO - 10 & & | \\
\hline & FIIE N20:43 IEN:1 - COP - File \#6 FANONIRE - 16, 17 & & i \\
\hline & File \#20 FANOMIR - 26,27 & & 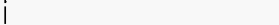 \\
\hline & |M10:29 - OOP - File \#6 FANOMIRL - 16, 17 & & \\
\hline & | File \#20 EPRONIRL - 26, 27 & & \\
\hline & SCL - File \#6 FANCIRL - 11 & & \\
\hline & | File \#20 EANOMIRL - 14 & & ] \\
\hline & PID - File \#6 FANONIRE - 10 & & 1 \\
\hline & File \#20 FAMONIRL - 12 & & i \\
\hline & |FIE M10:29 LEN:1 - COP - File \#6 FANONIRL - 16,17 & & 1 \\
\hline & | File \#20 FNONIRL - 26,27 & & \\
\hline & & PID OUIFU TO & \\
\hline & |FAN PLIO/MAN & FPN VFD & \\
\hline & $120: 41$ & $\Gamma_{-\infty}$ & |N10:29 - MOV - File \#20 FANOMRB \\
\hline 17 & $-1 / 1$ & Hogy File & 13 \\
\hline & 6 & || scunce $\quad$ \#NO0:43| & COP - File \#6 EPHONIRLE \\
\hline & {$[4]$} & | Dest $\quad$ HN10:29| & $16,17 \quad \ldots$ \\
\hline & & | Iergegth & |N20:43 - CAR - File \#6 FANONIRIE \\
\hline & & L L & 12 \\
\hline & & & COP - File \#6 FAnONIRLE \\
\hline & & PID AUIO/MAN & 16,17 \\
\hline & & STATUS & File \#20 FANOMBR \\
\hline & & No:0 & N10:0/1 - -( )- - File \#6 FANaE \\
\hline & & S & 17 \\
\hline & & 1 & File \#20 FANCE \\
\hline & |N10:29 - OOP - File \#20 FANONIKL - 26, 27 & & \\
\hline & i $\quad S C x-$ File \#6 EANONIRL - 11 & & \\
\hline & File \#20 BANanIRL - 14 & & \\
\hline & PID - File \#6 FANONIRG - 10 & & \\
\hline & File \#20 FANONIRL - 12 & & \\
\hline & FDE N10:29 InN:1 - COP - File \#6 FAMONIRL - 16,17 & & \\
\hline & File \#20 FANCNIRI - 26,27 & & \\
\hline & 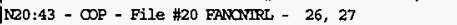 & & \\
\hline & |FILE ND0:43 IEN:100 - FFL - File \#13 FIFO - 10 & & \\
\hline & FILE ND0:43 IEN:1 - COP - File \#6 FANONIRL - 16, 17 & & \\
\hline & i File \#20 FanaNrr - 26,27 & & \\
\hline & N10:0/1 - - ( )- - File \#20 FANOMTI - 27 & & \\
\hline & COP - File \#6 FANONTL - 7 & & \\
\hline & File \#20 FANONIRL - 9 & & \\
\hline & PID - File \#6 FANONIRL - 10 & & \\
\hline & File \#20 FANONIRL - 12 & & \\
\hline & FIIE N10:0/1 IEN:1 - COP - File \#6 FANGNIR - 7 & & \\
\hline & File \#20 FANONIRL - 9 & & \\
\hline & FIIE M0:0/1 IEN:23 - PID - File \#6 FANONIRL - 10 & & \\
\hline & File \#20 Fancrirt - 12 & & \\
\hline & & PROPORTICAALI & \\
\hline & & GaIN & \\
\hline & & $\Gamma_{0}$ & IN10:3 - COP - File \#6 FMNONIRL $\mathrm{E}$ \\
\hline 18 & 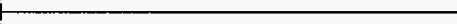 & foopy File & 7,18 \\
\hline & & |Saurce \#N36:13| & i File \#20 FANONIRE \\
\hline & & Dest \#MO:3| & N36:13 - COP - File \#6 FANOMRIE \\
\hline & & |Length 3 | & 7,18 \\
\hline & & 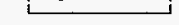 & FIIE N36:13 IEN:3 - \\
\hline & N10:3 - COP - File \#20 FANONIRL - 9 & & \\
\hline & FHE N10:3 IEN:23 - PID - File \#6 FANOMIL - 10 & & \\
\hline & File $\$ 20$ EPNaNIRL - 12 & & \\
\hline & FIIE N10:3 IEN: 3 - COP - File \#6 FANONIRL - 7, 18 & & \\
\hline & File \#20 FANONIRL - 9 & & \\
\hline & NB6:13 - COP - File \#6 FAMONIRL - 7, 18 & & \\
\hline
\end{tabular}




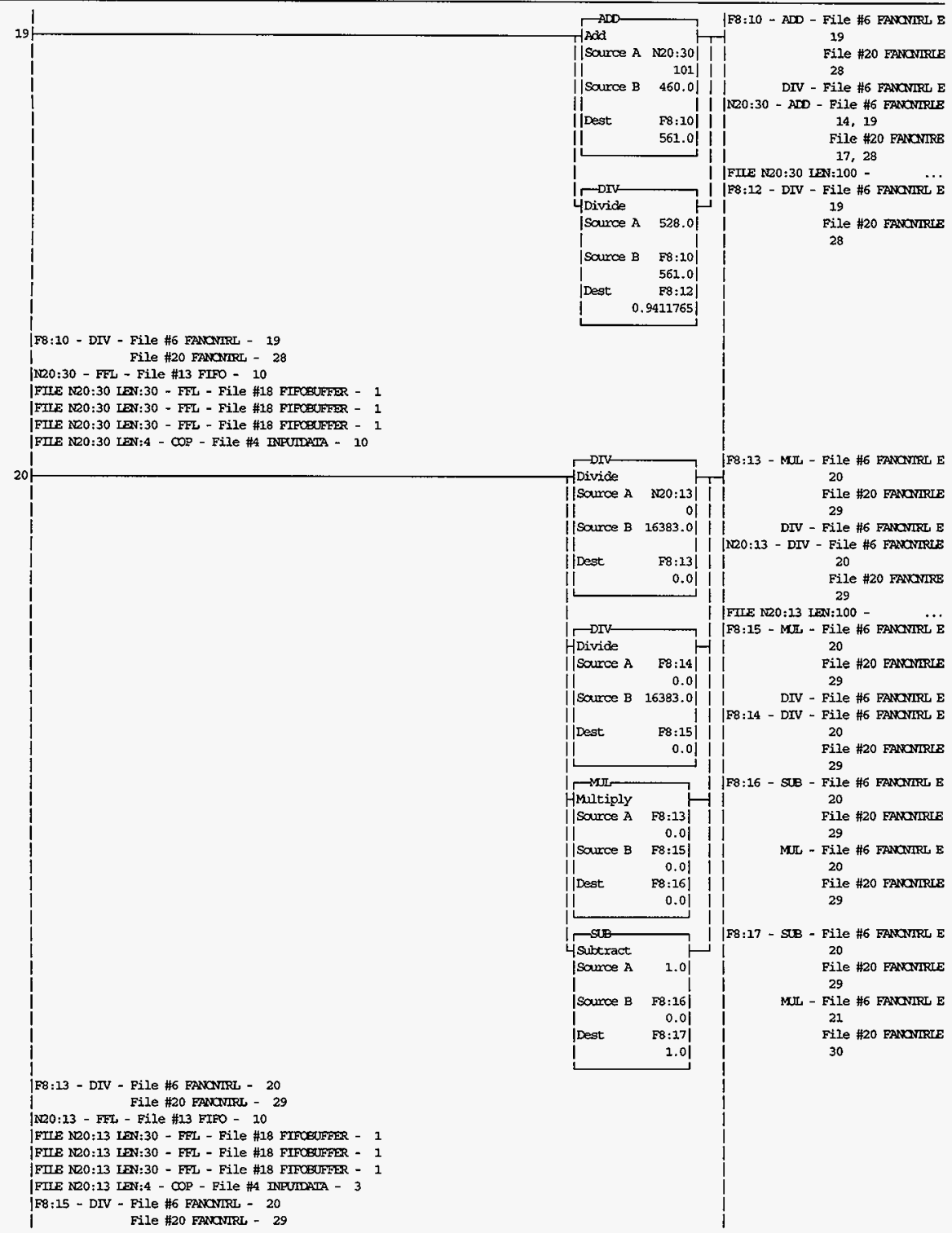




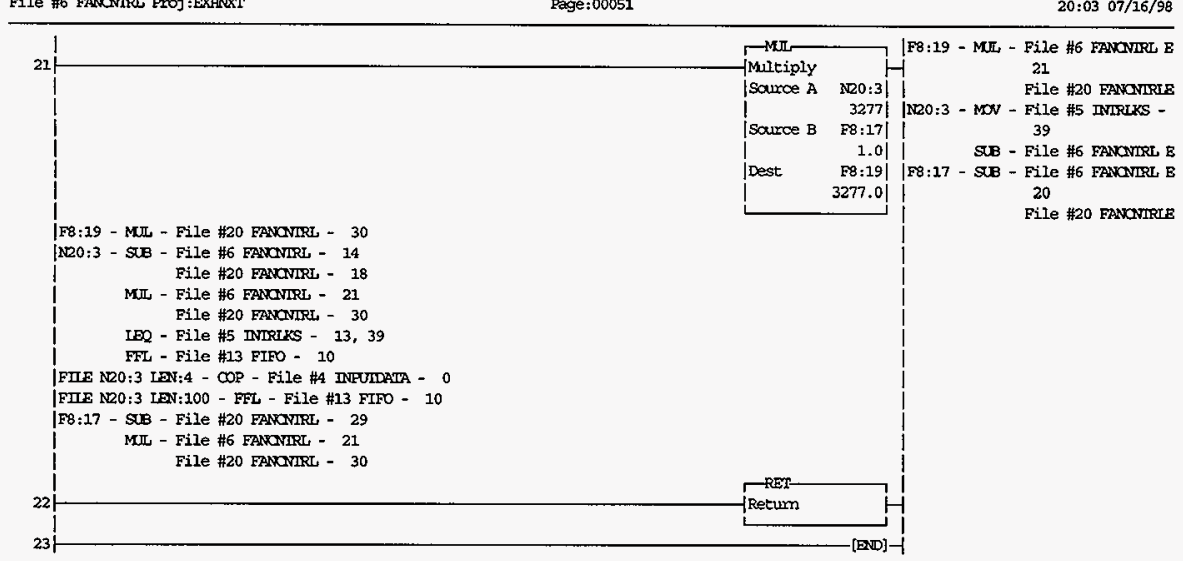

HNF-2934

Rev 0

A-51 
|r4:30.cn - 1 - File \#7 Meses $-14: 30.20-\dashv 1-1$ 1

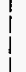

N20:41/2 - -( )- - File \#5 INRR $\dashv \vdash-$ File \#5 INRE
52,53
File \#6 FANCE |N13:0 - CIR - File \#2 MAII - 0 File \#3 STPRIUP 111

NOT - File \#2 MAN - 1 File \#3 SIARTUP |N12:0 - CR - File \#7 MESSIEN E 4

MOV - File \#7 Magavia E 1,9

N13:1 - NOT - File \#2 MAN - $\ddot{1}$ File \#3 SIPRTUP 2

MOV - File \#7 MESGVIEN E N12:1 - CIR - File \#7 MESGVIEN E 4

MOV - File \#7 MESGVIEN E 1,10

MM - FILE \#7 MESGIEN E 6

NEQ - File \#7 MESGIEN E 2

N12:2 - CIR - File \#7 MESGIEH E 4

MOV - File \#? MESGIIEN E 1,11

MM - File \#7 MESGIEN E 7

NEQ - File \#7 MESGIEN E
|N20:41/1 - $\vdash \vdash$ - File \#6 FRANOIRL - 5 File \#20 FANONIRL - 7

$-|/|-$ - File \#6 FANOMIR - 2 File \#9 SIMLATE - 1 File \$20 FANONIRI - 2 NRO:41/2 - † - File \#6 FANOVIRL - 5 1 File \#9 sMmLATE - 0 File \#20 FarrantrL - 7

|NDO:41/3 - -(U) - File \#9 SIMLATE - 0

$\dashv \vdash$ - File \#9 SIMLAIE - 1

$-/ / \vdash$ - File \#5 FaMONIRL - 4 File \#20 FANONIRL - 6

|NO0:41/5 - $\vdash$ - File \#5 INIRLKS - 48

N20:41/6 - -(L) - - File \#6 FANONIRL - 0 File \#20 EANONIRL - 0

-(U) - File \#6 FANONIRE - 1, 4 File \#20 FANONIRL - 1,6

$\dashv \vdash$ - File \#6 FANCNIRL - 16 File \#20 FARONIRL - 26

-1/ - File \#6 FANONIRL - 17 File \#20 FANONIRL - 27

in20:41/7 - $\vdash$ - File \#5 INIRLKS - 49

|ND0:41/8 - $\vdash$ - File \#6 FPNOARL - 13 File \#20 FMNOMPL - 16

|N20:41/9 - † - File \#5 INIRLKS - 50

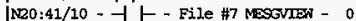
|N20:41/11 - $\vdash$ - File \#5 INIRLKS - 51 N20:41/12 - - ( )- - File \#5 INIRLKS - 67 | $-\mid-$ - File \#5 INTRIKS - 121 N20:41/13 - -( )- - File \#5 INIRIKS - 113 |N20:41/14 - -( )- - File \#5 INLPIKS - 114 |R20:41/15 - - ( ) - - File \#5 INLPLKS - 69

| $\rightarrow \vdash-$ FLle \#5 INIRUKS - 220 
|FIIE N20:41 IEN:100 - FFL - FIIE \#13 FIFO - 10

|N13:0 - NOT - File \#3 STARTUP - 2

FLL - File $\$ 2$ MAIN - 20

File \#3 STARTUP - 5

MM - File \#3 STARTUP - 11

File \#7 MESGUIEN - 1

File \#16 IMIX - 18, 23

|N13:0/0 - $-1 \vdash$ - File \#13 FIFO - 0, 1, 2, 3

File \#14 COUNIER - 0

FIIE N13:0 LEN:110 - FLL - File \#2 MAIN - 20

1 File $\$ 3$ STaraup - 5

|FIIE N13:0 LEN:3 - FLI - File \#3 STARTUP - 5

|FIIE NI3:0 LEN:4 - ELI - File \#3 STARTUP - 5

|FILE N13:0 LEN:7 - FLI - FIIe \#2 MRIN - 20

I File \#3 SIARTUP - 5

|N2: : 0 - MM - File \#7 MESGVIEN - 5

1 NEQ - File \#7 NESGIIEN - 2

|m12:0/0 - - 1 - File \#5 MIRuKS - 70

|M2:0/1 - 1 - File \#5 IMRLKS - 71

IMI2:0/2 - -1 - File 45 INTRLKS - 72

N12:0/3 - - F - File \#5 INIRLKS - 73

M2:0/4 - - - - File \#5 INIRIKS - 74

M12:0/5 - - - - File \#5 INIRUKS - 75

IN12:0/6 - - F- File \#5 INIRIKS - 76

IN2:0/7 - -1 - File \#5 INIRIKS - 77

[N2:0/8- $-1 \vdash-$ File \#5 INIRLKS - 78

WI2:0/9- $-1 \vdash$ - File 45 INIRIXS - 79

[N12:0/10 - † - File \#5 INLRLKS - 80

IN2:0/11 - - - File \#5 INIRLKS - 81

|N12:0/12 - $\dashv$ - File \#5 INIRLKS - 82

M12:0/13 - - - - File \#5 IMIRLXS - 83

|M12:0/14 - - - - File \#5 INIRLKS - 84

N12:0/15 - - 1- File \#5 INIRIKS - 85

N13:1 - MOV - File \#7 NESGVIEN - 1

f MM - File \#3 STARTUP - 4, 10

f File \#7 MESGVIEN - 5, 6, 7

FIIE N13:1 LEN:110 - FU - File \#2 MAIN - 20

File \#3 STARTü - 5

FIIE N13:1 LEN:3 - FLI - File \#3 STARTUP - 5

FIIE NI3:1 IEN:4 - FLU - File \#3 STARTUP - 5

|FIIE N13:1 LEN:7 - FLL - File \#2 MATN - 20

File \#3 STARTUP - 5

|M2:1/0 - † - File \#5 MMRUKS - 86

N12:1/1 - -1 - File \#5 INIPLS - 87

|N12:1/2 - $-1 \vdash-$ File \#5 INIRLKS - 88

N12:1/3 - $-1-$ - File \#5 INIRLKS - 89

|N12:1/4 - -| |- File \#S INIRLKS - 90

N12:1/5 - - $\vdash$ - File \#5 ININUKS - 91

[N]2:1/6 - -1 - File \#5 INIRIKS - 92

|N2:1/7 - - - - File \#5 INIRLKS - 93

[N12:1/8 - - 1 - File \#5 INIRLKS - 94

|N12:1/9 - $\dashv$ - File \#5 INIRIKS - 95

|N12:1/10 - - F - File \#5 IMIRIKS - 96

N12:1/12 - f - File \#5 INIRIKS - 97

N12:1/12 - - $\vdash$ - File \#5 INIRLKS - 98

N12:1/13 - - F - File \#5 INRIKS - 99

N22:1/14 - -1 - File \#5 DIIRLKS - 100

|N22:1/15 - † - File \#5 DNIRLKS - 101

N12:2 - NDO - Fille \#7 MESGIIN - 2

NL2:2/0 - $\dashv \vdash-$ File \#5 INTRIKS - 102

N12:2/1 - - - - File \#5 INIRUKS - 103

N12:2/2 - - - - Pile \#5 INIRIKS - 104

NM2:2/3 - - - - File \#5 INIRLKS - 105

N12:2/4 - - 1 - File \#5 INIRLKS - 106

|N22:2/5 - - - File \#5 INIRIKS - 107

|N22:2/6 - - $\vdash$ - File \#5 INLRIKS - 108

N12:2/7 - -1 - File \#5 INIRLKS - 109

N12:2/8 - - - - File \#5 INIRLX - 110

Na2:2/9 - -1 - File \#5 INIRIKS - 111

|M12:2/10 - †ト-File \#5 INIPLKS - 112 


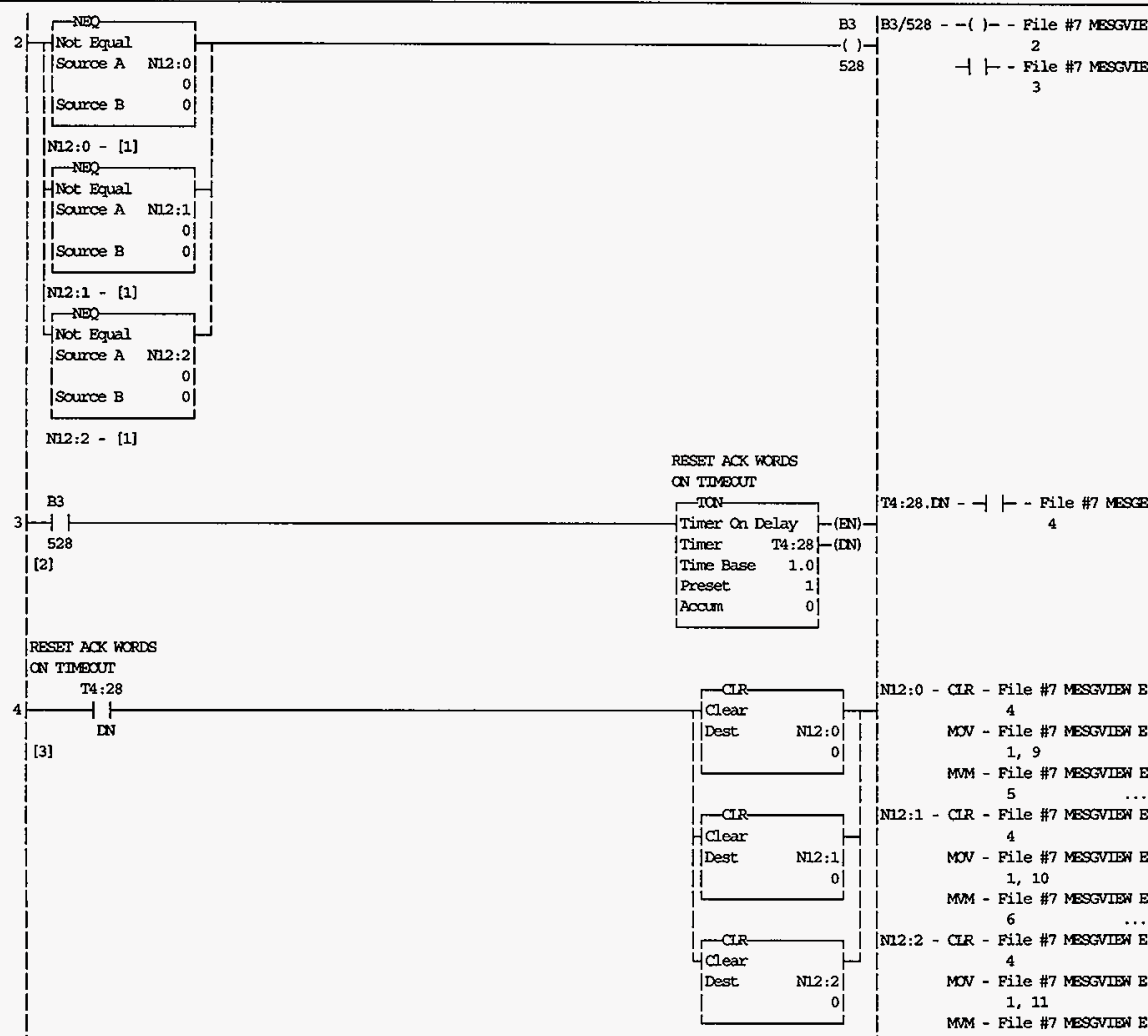

N12:0 - N囚 - File \#7 MESGIEN - 2 |N12:0\% - - 1 - File \#5 INIRLKS - 70 M12:0/1 - - F - File $\$ 5$ INIRLKS - 71 N12:0/2 - $\dashv$ - File \#5 INIRLXS - 72 |N12:0/3 - $\dashv$ - File \#5 INLRLKS - 73 M12:0/4 - -1 - - File \#5 INIRLKS - 74 |N2:0/5 - - 1 - File \#5 IMIRLKS - 75 [N2:0/6 - -1 - File \#5 INIRLS - 76 jn2:0/7 - -1 - File \#5 IMIRLK - 77 Im2:0/8 - -1 - File \#5 minuks - 78 Wr12:0/9 - - 1 - File \#S Mrrous - 79

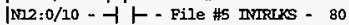
|M12:0/11 - $\dashv$ - File \#5 INIRLS - 81 |N12:0/12 - 1 - Eile \#5 INIRUKS - 82 ND2:0/13 - - - File \#5 INLRIKS - 83 |N12:0/14 - -1 F- File \#5 INLRLXS - B4 NM2:0/15 - † - - File \#5 INIRLKS - B5 |M2:1 - NE2 - File \#7 MESGIIEN - 2 M12:1/0 - t t- File \#5 INIRLKS - 86 |m2:1/1 - - $\vdash-$ File \#5 IMRLKS - 87 [N12:1/2 - -1 - File $\$ 5$ mIRUKS - 88 |N12:1/3 - $\dashv \vdash$ - File \#5 IMIRUKS - 89 N12:1/4 - - File \#5 INIRLKS - 90 |M2:1/5 - $\vdash$ - File \$5 INRLKS - 91 
|N12:1/6 - † - File \#5 INIRLKS - 92 |N12:1/7 - -f $\vdash$ - File \#5 INIRIKS - 93 m12:1/8 - - F - File \#5 INIRIKS - 94 IN12:1/9 - $\dashv 1-$ - File \#5 INIRIKS - 95 [M12:1/10 - オト - File $\$ 5$ INIRTS - 96 m2:1/11 - $\dashv$ - File \#5 INIRLKS - 97 [M12:1/12 - † - File \#5 INIRIKS - 98 |M2:1/13 - † - File \#5 IMIRLXS - 99 |N12:1/14 - -1 - File \#5 INIRLKS - 100 M2:1/15 - f - - File \#5 IMRLLS - 101

|M12:2 - MW - File \#7 MESGVIEN - 7 NEQ - File \#7 NESGVIEW - 2

|N12:2/0 - - $\vdash$ - File \#5 INIRIKS - 102 IN12:2/1 - -1 - File \#5 INIRLKS - 103 N12:2/2 - -1 - File \#5 IMRLKS - 104 NI2:2/3 - -1 - File \#5 MIIRLKS - 105 |N12:2/4 - - - File \#5 mMRIKS - 106 |N12:2/5 - $\dashv$ - File \#5 IMIRLXS - 107 |N12:2/6 - $\dashv$ - File \#5 IMIRLXS - 108 |N12:2/7 - 1 - File \#5 INIRLKS - 109 ND2:2/8 - - 1 - File \#5 INIRLKS - 110 |N12:2/9 - -1 - File \#5 INIRIKS - 111 N12:2/10 - †十 - File \#5 TNIRIKS - 112 |I:12.5 | 15

15

[ [8]

$\rightarrow M M$ Masked Mo

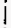

N12:0 - MM - File \#7 MESCVIEN - 5

NEQ - File \#7 MESGVIEW - 2

N12:0\% - $\vdash$ - File \#5 INIRIKS - 70 |N12:0/1 - -1 - File \#5 INIRLXS - 71 N12:0/2 - $-11-$ Eile \#5 INIRLKS - 72 1M2:0/3 - 1 - Eile \#5 INRLKS - 73 NN12:0/4 - - 1 - File \#5 INIRLKS - 74 |N12:0/5 - |N12:0/6 - -1 - File \#5 INIRIKS - 76 |N12:0/7 - -1 - File \#5 INIRLKS - 77 |N12:0/8 - 1 - - Eile \#5 INTRKS - 78 |N12:0/9 - $\dashv \vdash$ - File \#5 INIRLKS - 79 |N12:0/10 - †十- File \#S INIRLKS - 80 |N]2:0/11 - - F - File \#5 INIRLKS - 81 |N12:0/12 - † - File \#5 INIRUKS - 82 N12:0/13 - -1 - File \#5 INIRLKS - 83 |N12:0/14 - $1-$ - File \#5 INIRLK - 84 |N12:0/15 - - $\vdash$ - File \#S INIRLKS - 85 ND3:1 - MM - File \#3 SIARIUE - 4, 10

$$
\text { File \#7 MESGIIE - 5, 6, } 7
$$

Mask

IDest

N12:0 - CIR - File \#7 MESGVIr E 4 MOV - File \#7 MESGIFW E 1,9

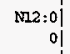
$\mathrm{N} 13: 1$

11
$-1 \mid$ $8000 \mathrm{~h}$ N13:1 - NOT - Eile \#2 MAT - 1 File \#3 smartup 2

MON - File \#7 MasGIEN B 1 
|N12:1/4 - A F - File \#5 nNIRLKS - 90 IM2:1/5 - $\vdash$ - File \#5 MIRLXS - 91 [N12:]/6 - - $\vdash$ - File \#5 INIRLXS - 92 |N32:1/7 - - | - File \#5 DNLIKS - 93 |MI2:1/8 - - $F$ - File \#5 ANIRIKS - 94 |N12:1/9 - - F - File \#5 DNIRIKS - 95

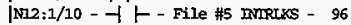
|N12:1/11 - - 1 - File \#5 INDRLS - 97 |N12:1/12 - - 1 - File \#5 IMLRKS - 98 |N12:1/13 - - F - File \#5 INIRLKS - 99 |N12:1/14 - - 1 - File \#5 INIRLKS - 100 IN2:1/15 - - - File \#5 INIRIkS - 101

|M13:1 - MM - File \#3 STARTUP - 4, 10 I File \#7 MESTIEW - 5, 6, 7 |FIIE ND3:1 IEN:110 - FUL - File \#2 MANN - 20 | File \#3 SIARIUP - 5 FIUE N13:1 IEN:3 - FLU - FIle \#3 SIARTUP - 5 |FIIE N13:1 IEN:4 - FUU - File \#3 SIARTUP - 5 |FIIE N13:1 IEN:7 - FIL - File \#2 MAN - 20 I |I:12.6 7) 1 3

[ [8] 1 1

N12:2 - MM - File \#7 MESGIIN - 7

NEQ - File \#7 NESGVIEN - 2

|N12:2/0 - - $\vdash$ - File \#5 INIRLKS - 102 |NI2:2/1 - - 1 - File \#5 ANTRLS - 103 |mi2:2/2 - - 1 - File \#5 DNIRIks - 104

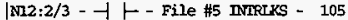
N12:2/4 - -1 - File \#5 DNIRLKS - 106 W12:2/5 - -1 - File \#5 INIRLKS - 107 |N12:2/6 - - $\vdash$ - File \#5 INIRLKS - 108 M12:2/7 - - - - File $\$ 5$ IMRUKS - 109 |N12:2/8 - - F - File 45 nNIRKs - 110 |N12:2/9 - -1 - File \#5 MNIFUKS - 111 |N12:2/10 - $\mid$ - File \#5 IMIRIS - 112 |N13:1 - MM - File \#3 SLARTUP - 4, 10 File \#7 MESGNIEW - 5, 6, 7 |FIIE N13:1 IEN:110 - FLL - File \#2 MAIN - 20 f File \#3 STARIUP - 5 |FIIE N13:1 InN:3 - FLL - File \#3 SIARTUP - 5 |FIE N13:1 EN:4 - FI - File \#3 SIARTUE - 5 |FIIE N13:1 IEN:7 - FLL - File \#2 MAIN - 20 | File $\# 3$ SITRIUP - 5

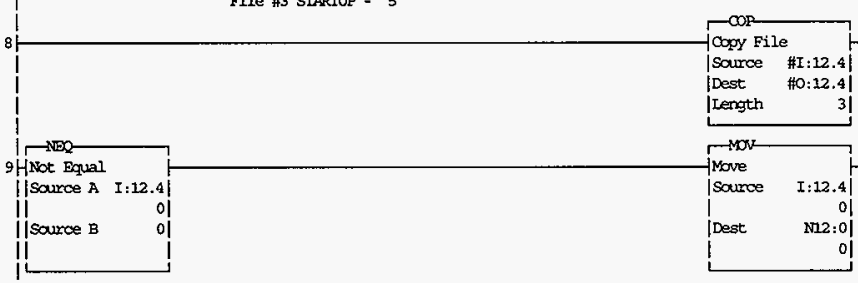

I:12.4 - MDN - File \#7 MESTIEAE 9 COP - File \#7 MESGIENB 8

NOQ - File \#7 MESGVIENE 9 M12:0 - CIR - File \#7 MESGIEN E 4

MOV - File \#7 MaSGIIEW E 1. 9 The

$[: 12.4-[9]$

1M12:0 - MM - File \#7 MESCVIEA - 5

1 NOQ - File \#7 MESGNIN - 2

N12:0/0 - -1 - File \#5 INIRLKS - 70 N12:0/1 - 1 - File \#5 INIRWS - 71 N12:0/2 - $1-$ - File \#5 INMUks - 72 N12:0/3 - - 1 - File \#5 INIRIKS - 73 N12:0/4 - - F - File \#5 INIRLS - 74 N12:0/5 - - $\vdash$ - File \#5 ININLKS - 75 |N12:0/6 - 
|N12:0/7 - †ト - File \#5 IMRrks - 77

M12:0/8 - - - File \#5 INTRLKS - 78

|N12:0/9 - - $\vdash$ - File \#5 INIRLS - 79

NB2:0/10 - $-1 \vdash$ - File \#5 INIRIKS - 80

|Na2:0/11 - $-1 \vdash$ - File \#5 INIRUKS - 81

N12:0/12 - $-1 \vdash$ - File \#5 INIRIKS - 82

|N12:0/13 - - |- File \#5 IMIRLKS - 83

|M12:0/14 - $\dashv$ - File \#5 IVIRUKS - 84

|M12:0/15 - $\vdash$ - File \#5 INIRUKS - 85

I:12.4 - NEQ - File \#7 MESGIEN - 9

|FIE I:12.4 IEN:3 - Cos - File \#7 MESGVIEN - 8 $1, \mathrm{NEO}$

10 HNot Equal

||Source A I: 12.5

II

|| Source B

II

1

N12:1 - MM - File \#7 MESGIDA - 6

NQQ - File \#7 MESGVID - 2

|N12:1/0 - - 1 - File \#5 INIRIKS - 86

N12:1/1 - - - File \#5 INIRLKS - 87

|M12:1/2 - $-\vdash$ - File \#5 INIRLKS - 88

N12:1/3 - - - File \#5 INIRTKS - 89

|M12:1/4 - $\vdash$ - File \#5 INIRLKS - 90

|N12:1/5 - - - File \#5 INIRLKs - 91

|N12:1/6 - - 1 - File \#5 INIRLKS - 92

|N12:1/7 - - F - File \#5 INIRLXS - 93

N12:1/8 - - F - File \#5 INIRUKS - 94

N2:1/9 - $\dashv \vdash$ - File \#5 IMIRLKS - 95

|N12:1/10 - $-\vdash$ - File \#5 INIRURS - 96

|N12:1/11 - - $\vdash$ - File \#5 INIFUKS - 97

N12:1/12 - $\dashv \vdash$ - File \#5 INIRIXS - 98

|N12:1/13 - † - File \#5 INIRLKS - 99

N12:1/14 - - $\vdash$ - File \#5 INIRLKS - 100

|N12:1/15 - オ † - File \#5 INIRIKS - 101

I:12.5/15 - †十 - File \#7 MESGVIBN - 5

|FIIE I:22,5 IDNF3 - COP - File \#7 MESGIEN - 8

Ir-NeQ-

11 Hot Equal

ISamoe A I:12.6

i| sance B

11

1:12.6 - [1]

N12:2 - MM - File \#7 MESGVIEN - 7

NEO - File \#7 MESGVIEN - 2

N12:2/0- $-1 \vdash-$ Pile \#5 INIRLKS - 102

|N12:2/1 - - - File \#5 INIRIKS - 103

|N12:2/2 - $1 \vdash-$ File \#5 INIRLXS - 104

N12:2/3 - 1 - File \#5 INIRIKS - 105

N12:2/4 - -1 1- - File \#5 INIRLKS - 106

N12:2/5 - - 1 - File \#5 INIRLXS - 107

|N12:2/5 - -1 - File \#5 INRLKS - 108

MI2:2/7 - - - - File \#5 IMIRLKS - 109

N12:2/8 - -1 - File \#5 INIRLK - 110

N12:2/9 - -1 - File \#5 INIRUs - 111

N12:2/10 - † $\vdash$ - File \#5 MNIRLKS - 112

I:12.6/2 - -1 - File \#7 MESGVISN - 6

1:12.6/3 - $\dashv \vdash-$ File \#7 MESGVIEN - 7

FIIE I:12.6 LEN:3 - COP - FIIJ \#7 MESOIEN - 8

1 GR:

12 HGreater Than $(A>B)$

|| Source A $T 4: 25 . A O C$

II

ilsource B

II source B

T4 :25.AOC - [14]

HNF-2934
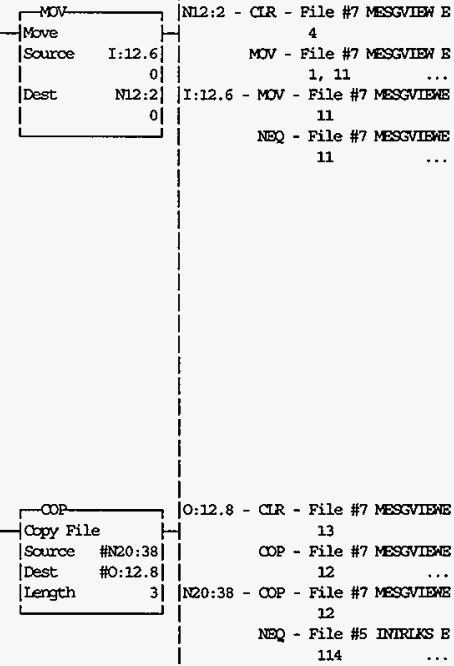


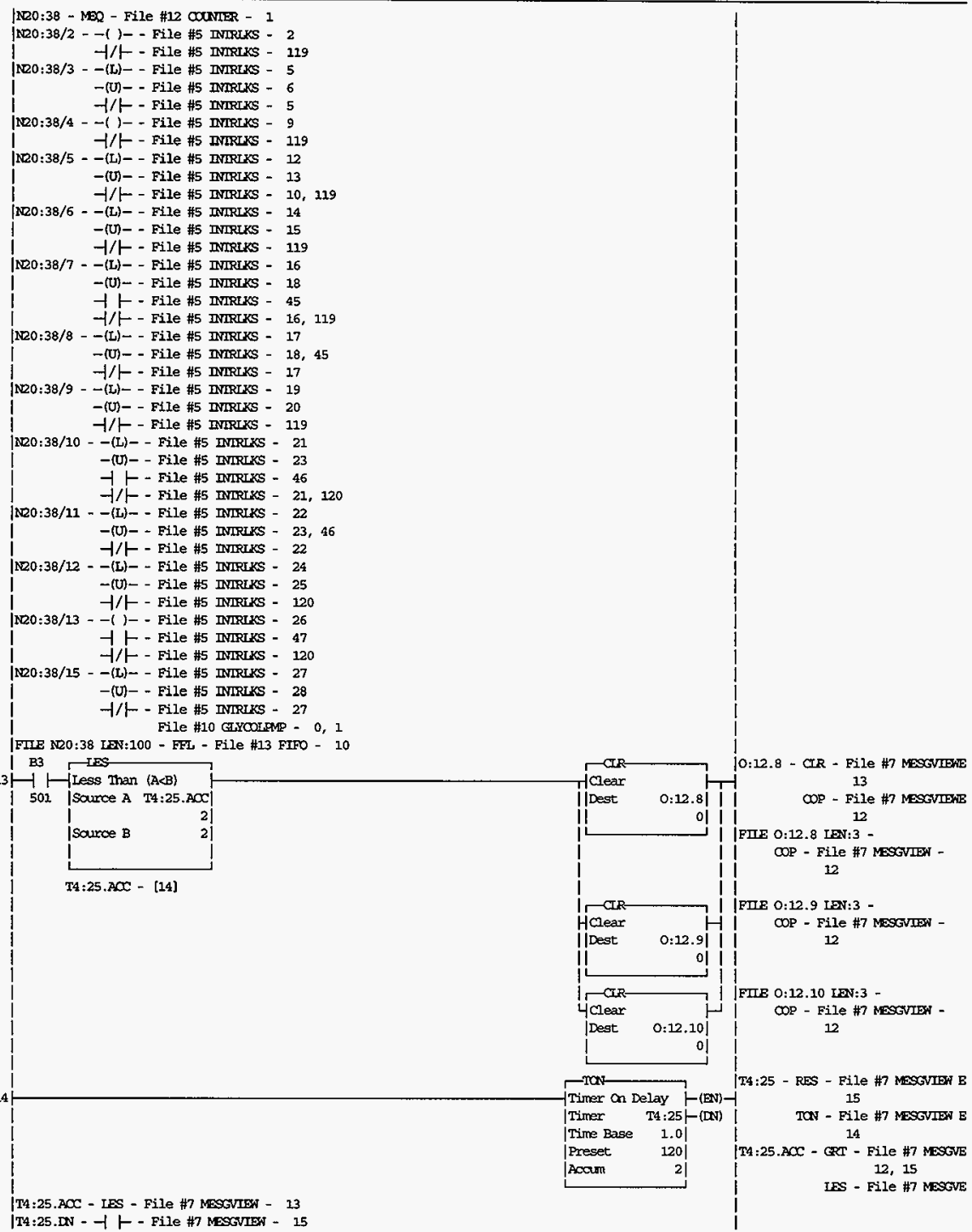

T4:25.DN - -1 1 - File \#7 MESGVIEN - 15 


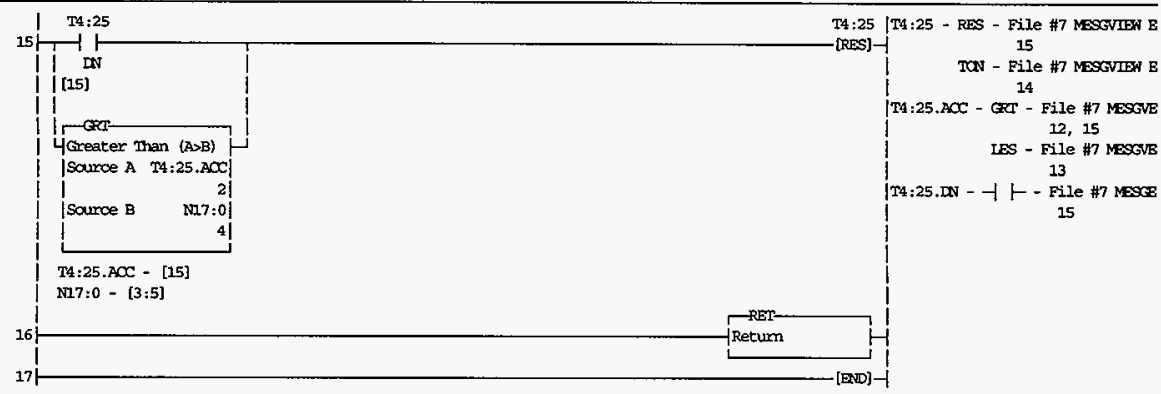

HNF-2934

Rev 0

A-59 
When this subroutine is active, it allows the user

to simulate a fan numing condition

†

IWW SEART

|

ND0:25/7 - - - - File \#S INIRtKS - 17, 19, 21, 22, 24, 52, 53, 58, 62, 66

File \#6 EAXONIRU - 0

File \#8 SYSSTAT - 1

File \#14 CONIER - 3

File \#16 INTLK - 0,6, 22, 24

File \#20 FANONR - 0

$-1 / 1-$ - File $\# 5$ MNRLiks - 15, 20, 25, 55, 57

File \#6 FMNONIL - 1,13

File \#8 SYSSTRT - 2

File \#16 INITK - 23

File \#20 FANONIRL - 1, 15

MM - File \#4 INPUIDATA - 12

File \#17 COPY OOR - 3

FIIE N20:25/7 IEN:100 - FFL - File \#13 FIFO - 10

FIIE N20:25/7 IEN:30 - FFL - File \#18 FIFCEUFER - 1

FIUE N20:25/7 IEN:30 - FEL - File \#18 FIFCEUEFER - 1

FIIE N20:25/7 IEN:30 - FFL - File \#18 FIFCBUFFER - 1

1N20:41/3 - $-1-$ - File \#9 SIMIIATE - 1

$-1 / F-$ File \#6 FaroNIRL - 4

File \#20 FANONIR - 6

MM - File \#7 MESGVIEN - 1

MM - File \#7 MLSGISN - 1
FIE N20:41/3 LEN:100 - FFL - File \#13 FIFO - 10

WH STOP
NeO:41

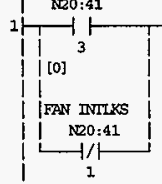

[5:121]

|Ne0:25/7 - - | - File \#6 FANONIRL - 0

| File \#8 SYSSTAT - 1

File \#14 OONIER - 3

File \#16 INII - 0, 6, 22, 24

File \#20 FPNONIRL - 0

$-1 / F$ - File \#5 INIRLKS - 15, 20, 25, 55, 57

File \#6 FANONIR - 1, 12

File \#8 SYSSTPT - 2

File \#16 INTK - 23

File \#20 FANONIR - 1, 15

MM - File \#4 INPUIDATA - 12

File \#17 OOF OrI - 3

FILE NLO:25/7 LEN:100 - FFL - File \#13 FIFO - 10

FIIE N20:25/7 LEN:30 - FFL - File \#18 FIFCBUEFER - 1

|FIIE N20:25/7 LEN:30 - FFL - File \#18 FIFOEUFFER - 1

|FIIE N20:25/7 LEN:30 - FFL - File \#18 FIFCBUFFER - 1

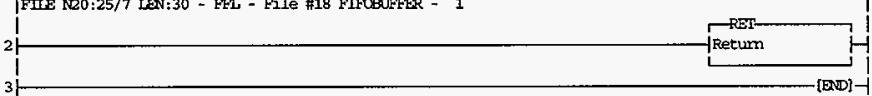




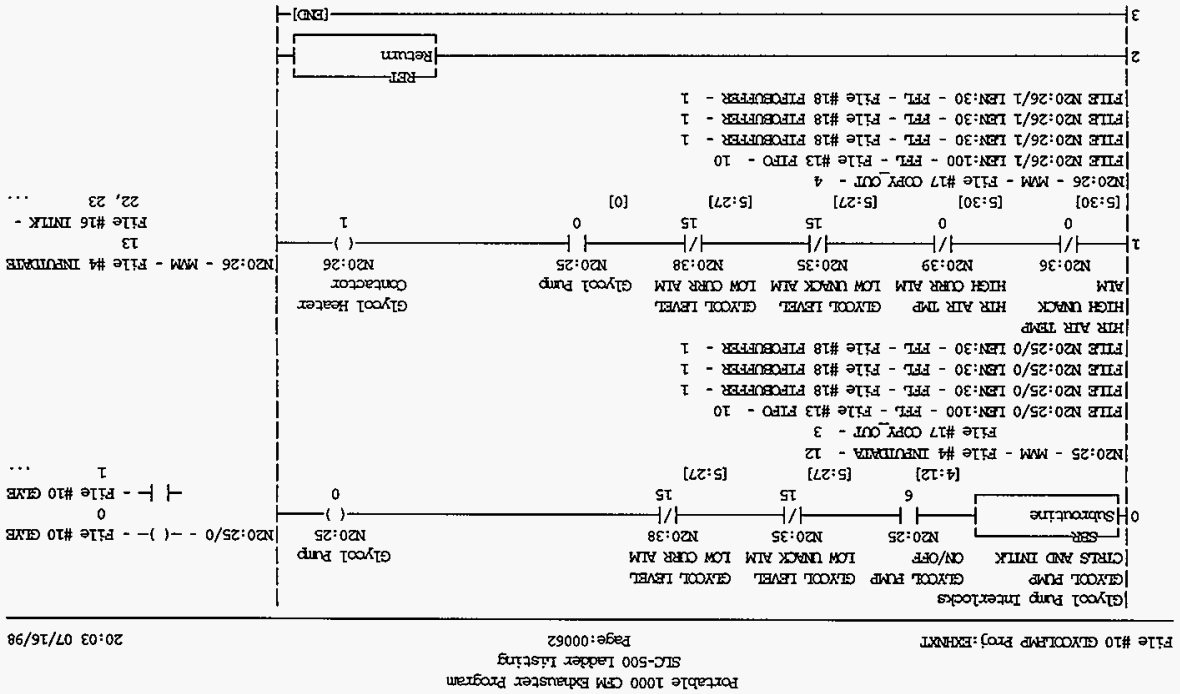




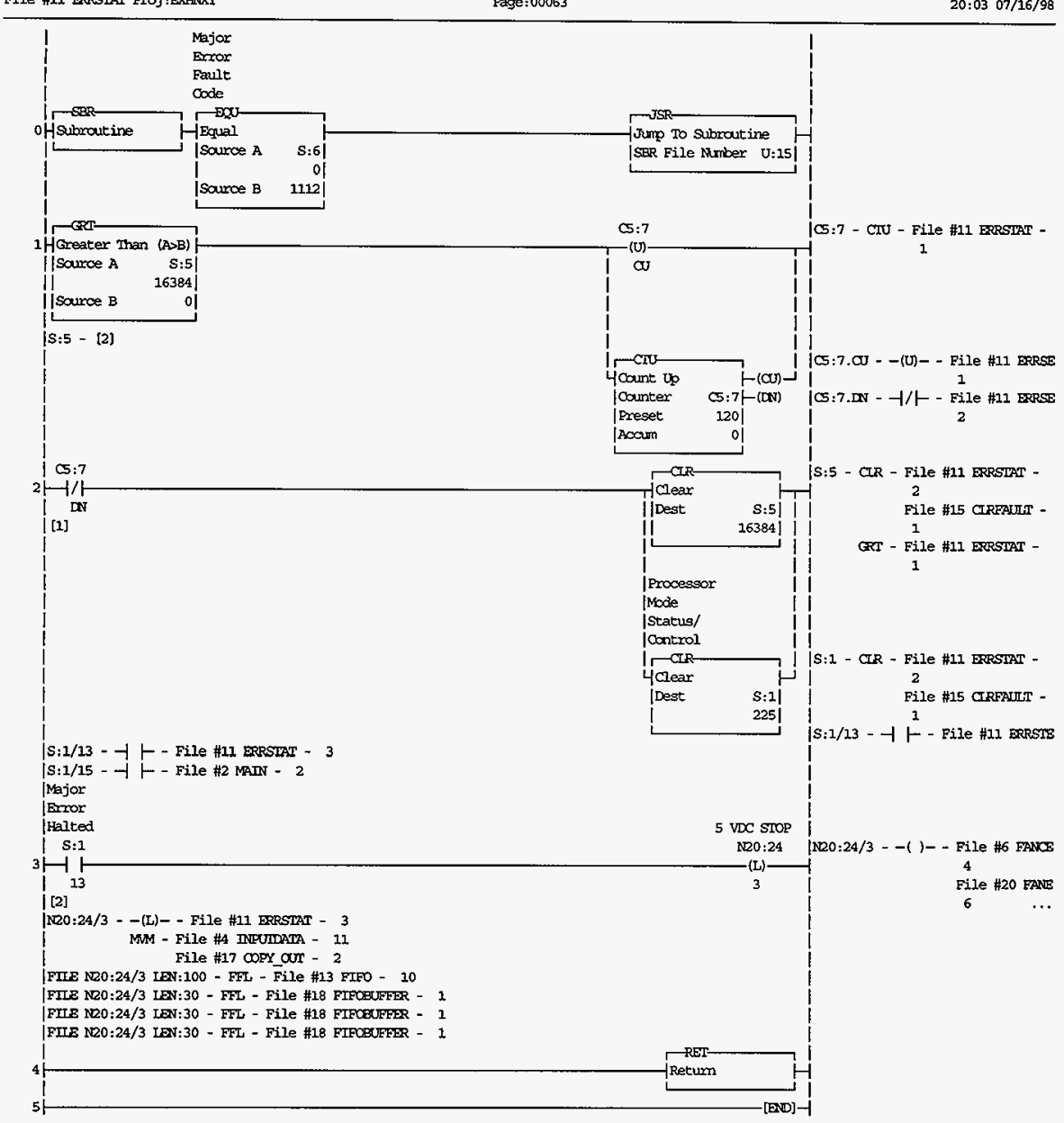




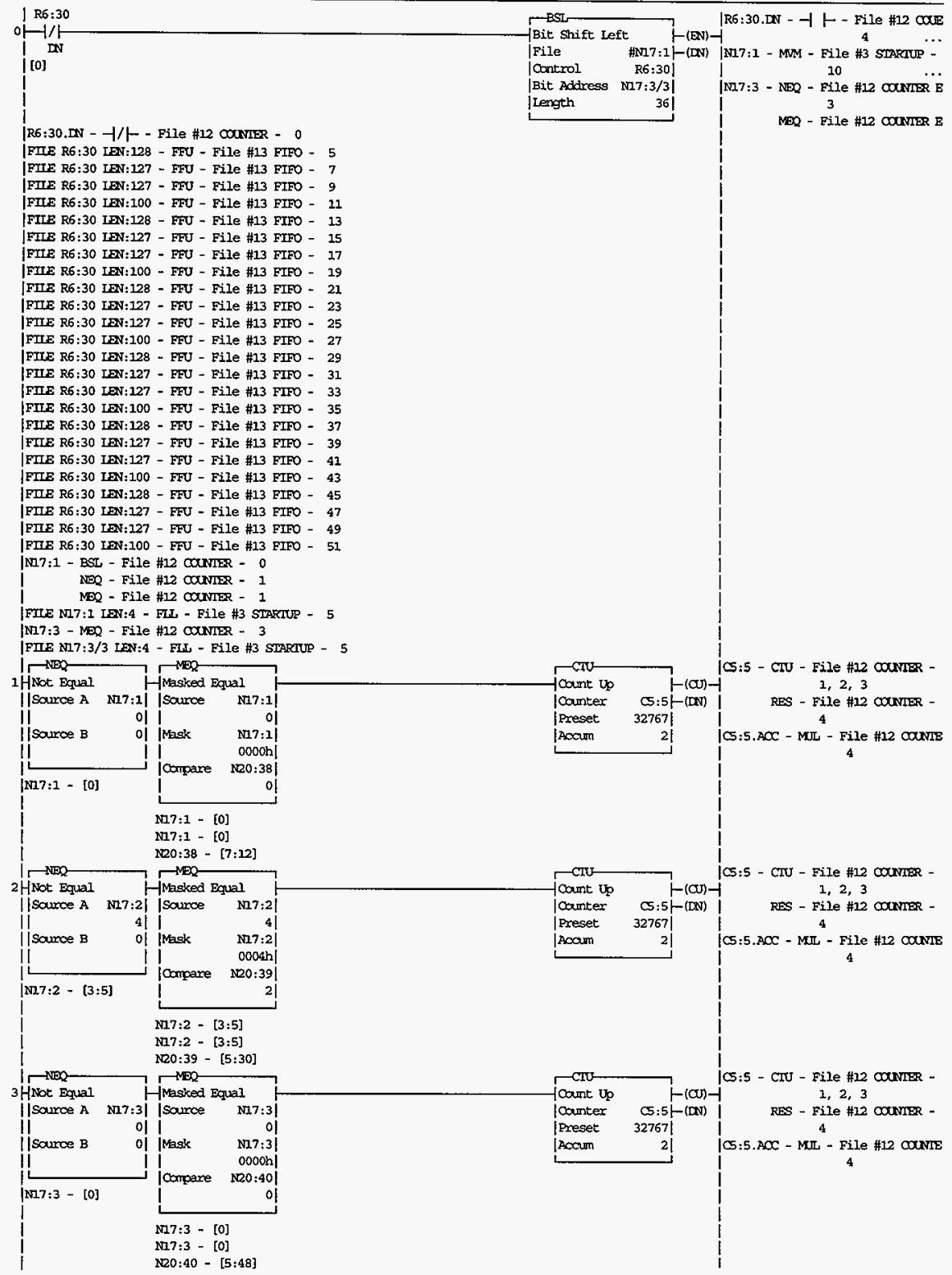




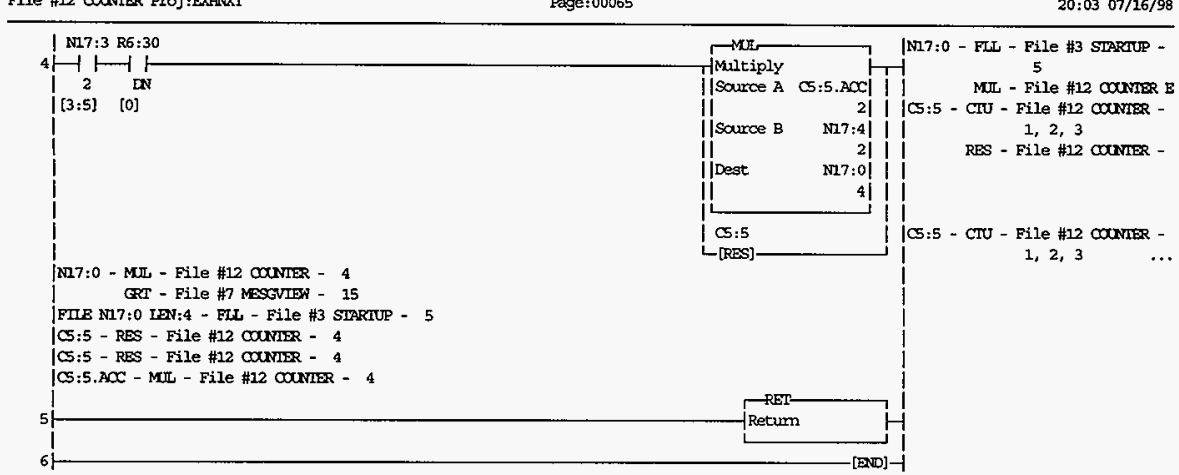




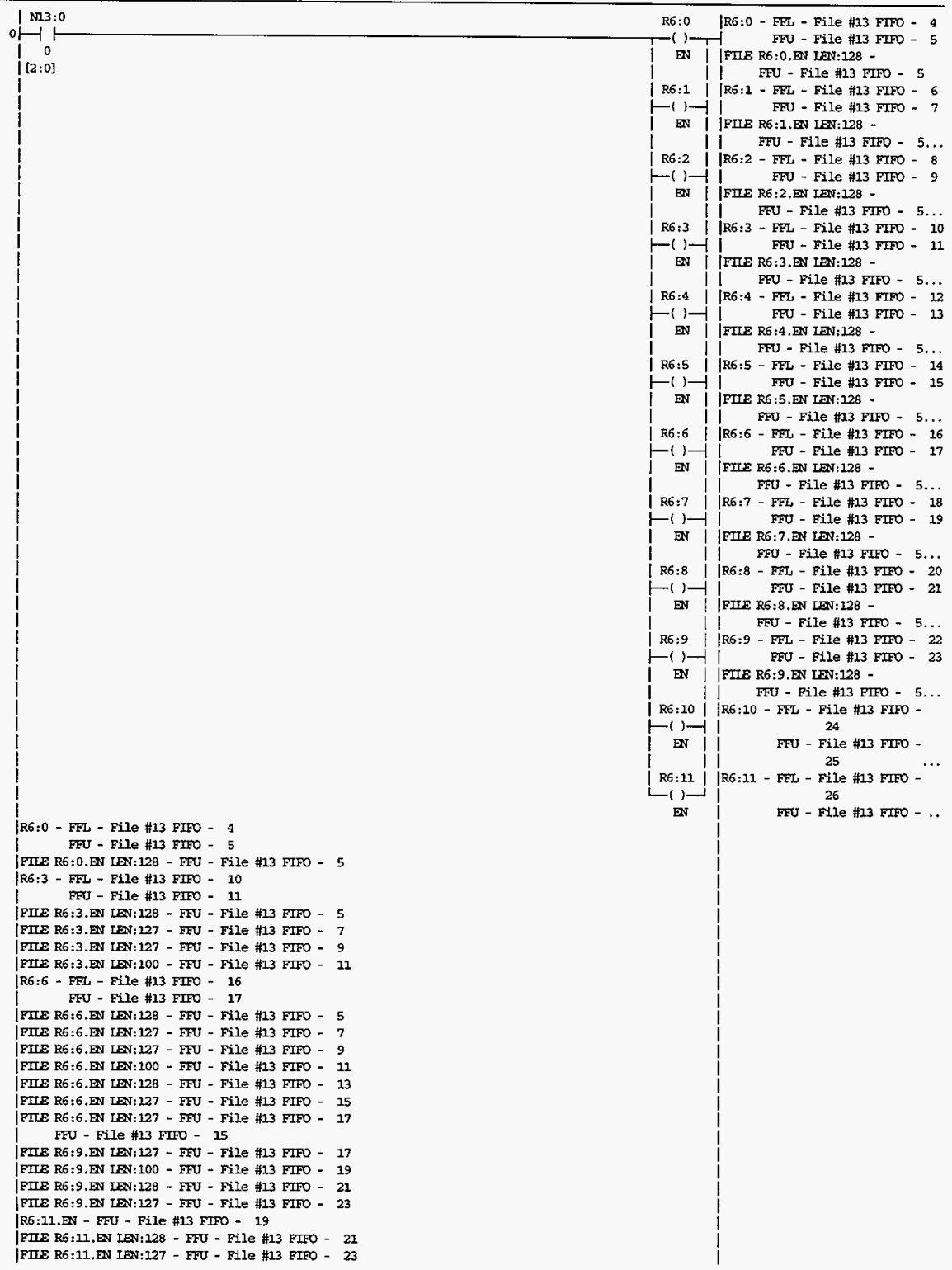




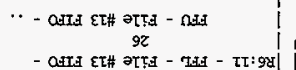

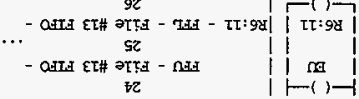

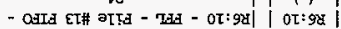

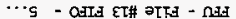

- 8ZT:NAT NA $6: 98$ Tास.

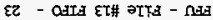

Z2 - OJIJ हТ\# әโT] - TI3 - 6:98|

‥s - OALS हा\# әTT - กसG

- 8Zा:NGI IA 8:98 GHAI

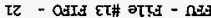

‥ - a

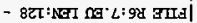

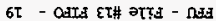

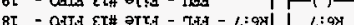

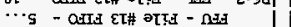

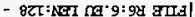

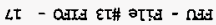

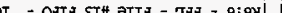

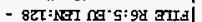

ST - OIIA हז\# әTाज - กAS

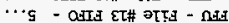

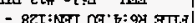

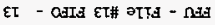

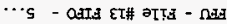

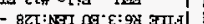

IT - GIL ET\# РTTS - RA3

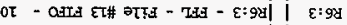

$\cdots 5$ - OAIA EI\# गITI - RUA

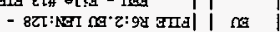

6 - वान हा\# गार - กस ।

8 - СाI हट\# әाT - TAS - $2: 94 \mid$ | $z: 94$

$\cdots s$ - OIII हात aTts - nas

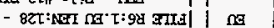

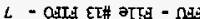

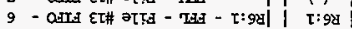

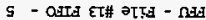

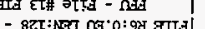

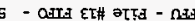

b - OIII ET\# әTTI - THI - 0:98

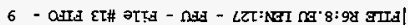

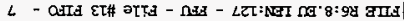

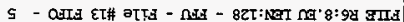

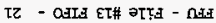

OZ - OAI हा\# әTTa - THA - 8:9a

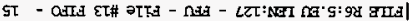

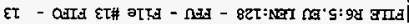

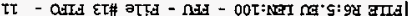

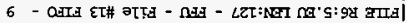

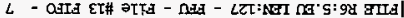

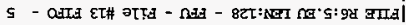

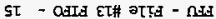

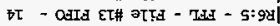

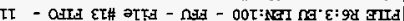

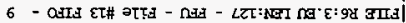

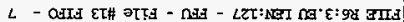

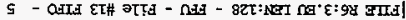

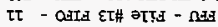

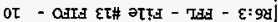

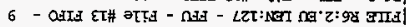

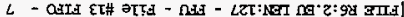

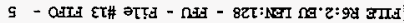

6 - OAId EL\# әTTA - RUA

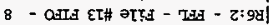


|FILE R6:8.EU IEN:100 - FFU - File \#13 FIFO - 11 FHB R5:8.EU IEN:128 - FFU - File \#13 ETFO - 13 |FILE R6:8.EU IEN:127 - FFU - File \#13 FIFO - 15 |FIIE R6:8.EU IEN:127 - FNU - Eile \#13 FIFO - 17 |FIIE R6:8.DU IDN:100 - FTU - File \#13 FIFO - 19 FIIE R6:8.DU IEN:128 - FEU - File \#13 FIFO - 21 N13:0

21

10

| $[2: 0\}$

R: $6: 12$

R6:12 - FFL - File \#13 FIFO $-(1)-1$

11

28

FFU - File \#13 FIFO -

29

|FIL R6:12.EN LEN:128 -

R6:13 | |R6:13 - FFL - File \#13 EIFO -

$\longrightarrow$ ( ) 1 - 30

EN $\mid$ FFO - File \#13 FIFO -

R6:14 |R6:14 - FFL - File \#13 FIFO -

EN I I FFU - File \#13 FIFO -

R6:15 |RE:15 - FFL - File \#13 FIFO -

( ) 1 | 34

EN | FFU - File $\$ 13$ FIFO -

R6:16 |R6:16 - EFL - File \#13 FIFO -

$-1)-1$ ( 16

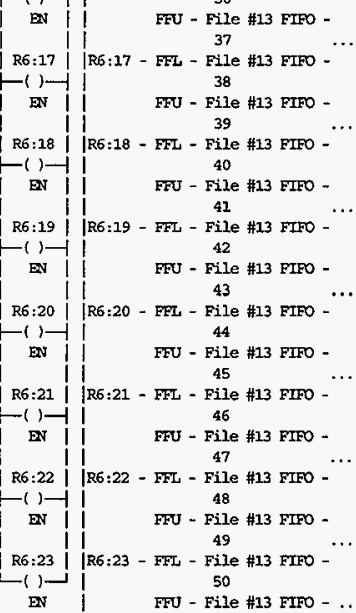

FFU - File \#13 FIFO - 21

|FHE R6:14. DN IEN:127 - FFU - File \#13 FIFO - 23 |FIIE R6:14.EN LEN:127 - FFU - File \#13 FIFO - 25 |FITE R6:14.EN IEN:100 - FFU - File \#13 FIFO - 27 |FIIE R6:14.EN IEN:128 - FWU - File \#13 FIFO - 29 |FIE R6:14.EN IEN:127 - FEU - File \#13 FIFO - 31 |FIE R6:14.EN IEN:127 - FRU - File \#13 FIFO - 33 FFU - File \#13 FIFO - 13

|FIJE R6:15. EN IEN:127 - FFU - File \#13 FIFO - I5 |FIIE R6:15.EN IEN:127 - FFU - File \#13 FTFO - 17 |FIIE R6:15.EN LEN:100 - FFU - File \#13 FTFO - 19 |FIE R6:15.EN LEN:128 - FFU - File \#13 FIFO - 21 |FIE R6:15.EN IEN:127 - FFU - File \#13 FIFO - 23 |FTE R6:15.EN IAN:127 - FFU - File \#13 FIFO - 25 |FTIE R6:15.EN IDN:100 - FFU - File \#13 FIFO - 27 |FTE R6:15.DN IEN:128 - FFU - File \#13 FIFO - 29 |FIE R6:15.EN LEN:127 - FFU - File \#13 FIFO - 31 |FTE R6:15.EN IEN:227 - FFU - File \#13 FIFO - 33 |FIIE R6:15.EN IEN:100 - FFU - File \#13 FIFO - 35 
|R6:16 - FFL - File \#13 FIFO - 36

| FWU - File \#13 FIFO - 37

FIIE R6:16. EN IEN:128 - FFU - File \#13 FIPO - 5 FITE R6:16. EN IDN:127 - FEU - FIle \#13 FIFO - 7 FIIE R6:16.DN IEN:127 - FEU - File \#13 FIFO - 9 |FIIE R6:16.EN LEN:100 - FEU - File \#13 FIFO - 11 FIE R6:16.EN LEN:128 - FFU - File \#13 FIFO - 13 |FIIE R6:16.EN IEN:127 - FFU - File \#13 FIFO - 15 |FIE R6:16.DN IEN:127 - FFU - File \#13 FIFO - 17 |FTE R6:16.EN IDN:100 - FFU - File \#13 FIFO - 19 |FIE R6:16.EN IEN:128 - FFU - File \#13 FIFO - 21 |FIE R6:16.EN IEN:127 - FFU - File \#13 FIFO - 23 |FIE R6:16.EN IEN:127 - FFU - File \#13 FIFO - 25 FIIE R6:16.EN IEN:100 - FNU - File \#13 FIFO - 27 FIE R6:16.EN IEN:128 - FEU - File \#13 FIFO - 29 FIE R6:16. BN IEN:127 - FVU - File \#13 FIFO - 31 |FIIE R6:16.EN IDN:127 - FEU - File \#13 FIFO - 33 |FIIS R5:16.EN LEN:100 - FFU - File \#13 FIFO - 35 FIL R6:16.EN LEN:128 - FFU - File \#13 FIFO - 37 FFU - File \#1.3 FIFO - 27

FIIE R6:17. EN IEN:128 - FFU - File \#13 FIFO - 29 JEIIE R6:17.EN IEN:127 - FFU - File \#13 FIFO - 31 |FILE R6:17.EN LEN:127 - FRU - File \#13 EIFO - 33 |FIB R6:17.EN LEN:100 - FFU - File \#13 FIFO - 35 |FIIE R6:17.EN LEN:128 - FFU - File \#13 FIFO - 37 FIIE R6:17.EN IEN:127 - FFU - File \#13 FIFO - 39 |R6:21 - FFL - File \#13 FIFO - 46

| FFU - File \#13 FIFO - 47

|FIIE R6:21.EN IEN:128 - FFU - File \#13 FIFO - 5 |FIIE R6:21.EN IEN:127 - FFU - File \#13 FIFO - 7 FIIE R6:21. EN LEN:127 - FFU - File \#13 FIFO - 9 |FILE R6:21.EN LEN: 100 - FFU - File \#13 FIFO - 11 |FIIE R6:21.EN IEN:128 - FFU - File \#13 FIFO - 13 |FItE R6:21.EN LEN:127 - FWU - File \#13 FIFO - 15 |FIIE R6:21. EN LEN:127 - FRU - File \#13 FIFO - 17 |FIIE R6:21. EN LEN:100 - FFU - File \#13 FIFO - 19 |FIIE R6:21.EN LEN:128 - FFU - File \#13 FIFO - 21 |FUE R6:21. EN LEN:127 - FFU - File \#13 FTFO - 23 |FIIE R6:21.EN LEN:127 - FFU - File \#13 FIFO - 25 |FIIE R6:21. EN LDN:100 - FFU - File \#13 FIFO - 27 |FIIE R6:21, EN LDN:128 - FFU - File \#13 FIFO - 29 |FIIE R6:21.EN LEN:127 - FFU - File \#13 FIFO - 31 |FIIE R6:21.EN LEN:127 - FFU - File \#13 FIFO - 33 FIIE R6:21. EN IEN:100 - EFU - File \#13 FIFO - 35 |FIIE R6:21.EN LAN:128 - FFU - File \#13 FIFO - 37 |FIIE R6:21.EN INS:127 - FFU - File \#13 FIFO - 39 |FIE R6:21, EN LEV:127 - FFU - File \#13 FIFO - 41 |FHE R6:21.EN EDV:100 - FFU - File \#13 FIFO - 43 |FIIE R6:21.EN EEN:128 - FFU - File \#13 FIFO - 45 |FILE R6:21.EN LEN:127 - FFU - File \#13 FIFO - 47 FFU - File \#13 FIFO - 11

FTIE R6:23. EN IEN:128 - FF - File \#13 FIFO - 13 |FIIE R6:23. EN LEN:127 - FWU - Bile \#13 FIFO - 15 |FIE R6:23.EN LEN:127 - FVU - File \#13 FIFO - 17 FIIE R6:23. DN LEN:100 - FFU - File \#13 FIFO - 19 FIIE R6:23.EN LEN:128 - FFU - File \#13 FIFO - 21 |FIEE R6:23. EN LEN:127 - FFU - File \#13 FIFO - 23 [FIIE R6:23.EN IEN:127 - FFU - File \#13 FIFO - 25 |FIE R6:23. EN LEN:100 - FFU - File \#13 FIFO - 27 |FIIE R6:23. EN LEN:128 - FFU - File \#13 FIFO - 29 |FIIE R6:23.EN LEN:127 - EFU - File \#13 FIFO - 31 FIIE R6:23,EN LEN:127 - FFU - File \#13 FIFO - 33 |FIIE R6:23. BN LEN:100 - FFU - File \#13 FIFO - 35 FIIE R6:23. EN IEN: 128 - FFU - File \#13 FIFO - 37 |FIIE R6:23.EN IEN:127 - FFU - File \#13 FIFO - 39 FIIE R6:23.EN IEN:127 - FFU - File \#13 FIFO - 41 |FIIE R6:23. EN LEN:100 - FFU - File \#13 FIFO - 43 FIIE R6:23.EN LDN:128 - FFU - File \#13 FIFO - 45 FIII R6:23.EN IDN:127 - FFU - File \#13 FIFO - 47 |FHE R6:23.EN LEN:227 - FFU - File \#13 FIFO - 49 |FIE R6:23,EN LEN:100 - FFU - File \#13 FIFO - 51 


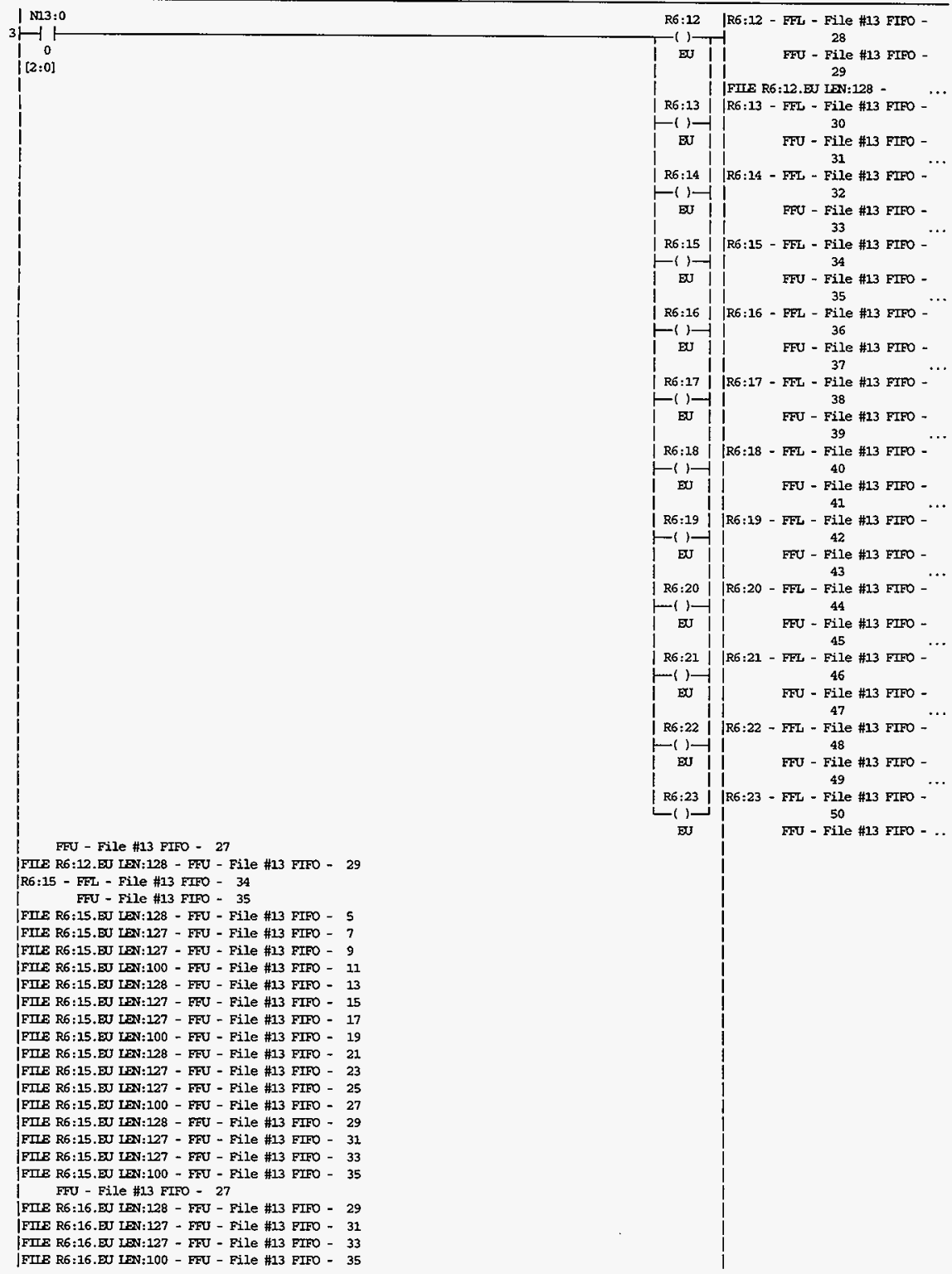


|FIIE R6:16.EU IFN:128 - FWU - File \#13 FIFO - 37 FFU - File \#13 FIFO - 23

|FIE R6:18. EU IDN:127 - FFU - File \#13 FIFO - 25 |FIIE R6:18.DU IEN:100 - FFU - File \#13 FIFO - 27 |FIIE R6:18.DU LEN:128 - FNU - File \#13 FIFO - 29 |FIE R6:18.EU LEN:127 - FEU - File \#13 FIFO - 31 FIE R6:18.EU IEN:127 - FEU - File \#13 FIFO - 33 |FIIE R6:18.DU IEN:100 - FUU - File \#13 FIFO - 35 |FIE R6:18.W IDN:128 - FFU - File \#13 FIFO - 37 |FIIE R6:18.EU IEN:127 - FFU - File \#13 FIFO - 39 |FUE R6:18.EU LDN:127 - FFU - File \#13 FIFO - 41 FFU - File \#13 FIFO - 25

[FIE R6:20.EU IEN:100 - FFU - File \#13 FIFO - 27 |FTE R6:20.DU IEN:128 - FEU - File \#13 FTFO - 29 |FIIE R6:20.EU IEN:127 - FTU - File \#13 FIFO - 31 FUE RG:20.EJ LEN:127 - FFU - File \#13 FTFO - 33 FUE R6:20.EJ IEN:100 - FFU - File \#13 FIFO - 35 FIIE R6:20.EU IEN:128 - FFU - File \#13 FIFO - 37 FTIE R6:20. EJ IEN:127 - FFU - File \#13 FIFO - 39 |FTIE R6:20.DU IEN:127 - FFU - File \#13 FTFO - 41 |FIE R6:20.DU IEN:100 - FFU - File \#13 FIFO - 43 |FIE R6:20.DO IEN:128 - FFU - File \#13 FIFO - 45 |R5:23 - FFL - File \#13 FIFO - 50

| FFU - File \#13 FIFO - 51

FIIE R6:23. EU IEN:128 - FFU - File \#13 FIFO - 5 FIIE R6:23. HU IEN:127 - FFU - File \#13 FIFO - 7 |FIIE R6:23.DU IEN:127 - FWU - File \#13 FIFO - 9 |FILE R6:23.EU IDN:100 - FEU - File \#13 FINO - 11 |FIIE R6:23.EU IDN:128 - FEU - File \#13 FINO - 13 FIIE R6:23.EU IDN:127 - FFU - File \#13 FIFO - 15 FIIE R6:23.EU IEN:127 - FFU - File \#13 FIFO - 17 FIIE R6:23.BU IEN:100 - FFU - File \#13 FIFO - 19 |FTIE R6:23.BU IEN:128 - FFU - File \#13 FTFO - 21 |FIIE R6:23.EU IEN:127 - FFU - File \#13 FIFO - 23 |FIIE R6:23.WU IEN:127 - FFU - File \#13 FIFO - 25 |FIIE R6:23.EU LEN:200 - FFU - File \#13 FIFO - 27 |FIEE R6:23,EU LDN:128 - FFU - File \#13 FIFO - 29 |FIIE R6:23.EU IEN:127 - FTU - File \#13 FIFO - 31 |FIIE R6:23.WU LEN:127 - FFU - File \#13 FIFO - 33 |FITE R6:23. EU IEN:100 - FNU - File \#13 FIPO - 35 |FIIE R6:23, EU LDN:128 - FNU - File \#13 FIFO - 37 |FIIE R6:23.EU IDN:127 - FNU - File H13 FIFO - 39 FIIE R6:23.EU IEN:127 - FFU - File \#13 FIFO - 41 |FIIE R6:23. BU IEN:100 - FFU - File \#13 FIFO - 43 FIIE R6:23. EU LDN:128 - FFU - File \#13 FTFO - 45 |FIIE R6:23.DU IDN:127 - FFU - File \#13 FIFO - 47 |FIIE R6:23.EU IEN:127 - FFU - File \#13 FIFO - 49 FILE R6:23.EN LAN:100 - EFU - File \#23 FIFO - 51 1

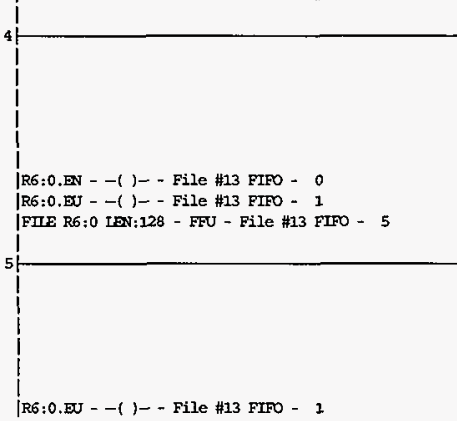

-FFI

FIFO Ioad isource |FIFO cantiol Length |Position Position
FTU FIFO [FIFO Dest. fOontrol IIength position pesition

\section{N23:328 (DN)} fस2 $3:$ 아-(EM) $\mathrm{R} 6: 0$ ) 128 1271 N20:58-(DI) R6:0। 1281 127
R6:0 - FFL - File \#23 FIPO - 4 FN - File \#13 FIFO - 5 . N23:128 - FFL - File \#13 FIPO 4 FFU - File \#13 FIFO 7

N23:0 - FFL - File \#13 FTFO - 4 FFU - File \#13 FIFO - 5

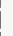

R6:0 - FFL - File \#13 FIFO - 4 FFU - File \#13 FIFO - 5 \#N23:0-(DN) |R6:0.EN - - ( )- - File \#13 FIFOE FVU - File \#13 FIFO - 5 |FITE N20:58 LEN: 100 FFL - File \#23 FIPO - 10 


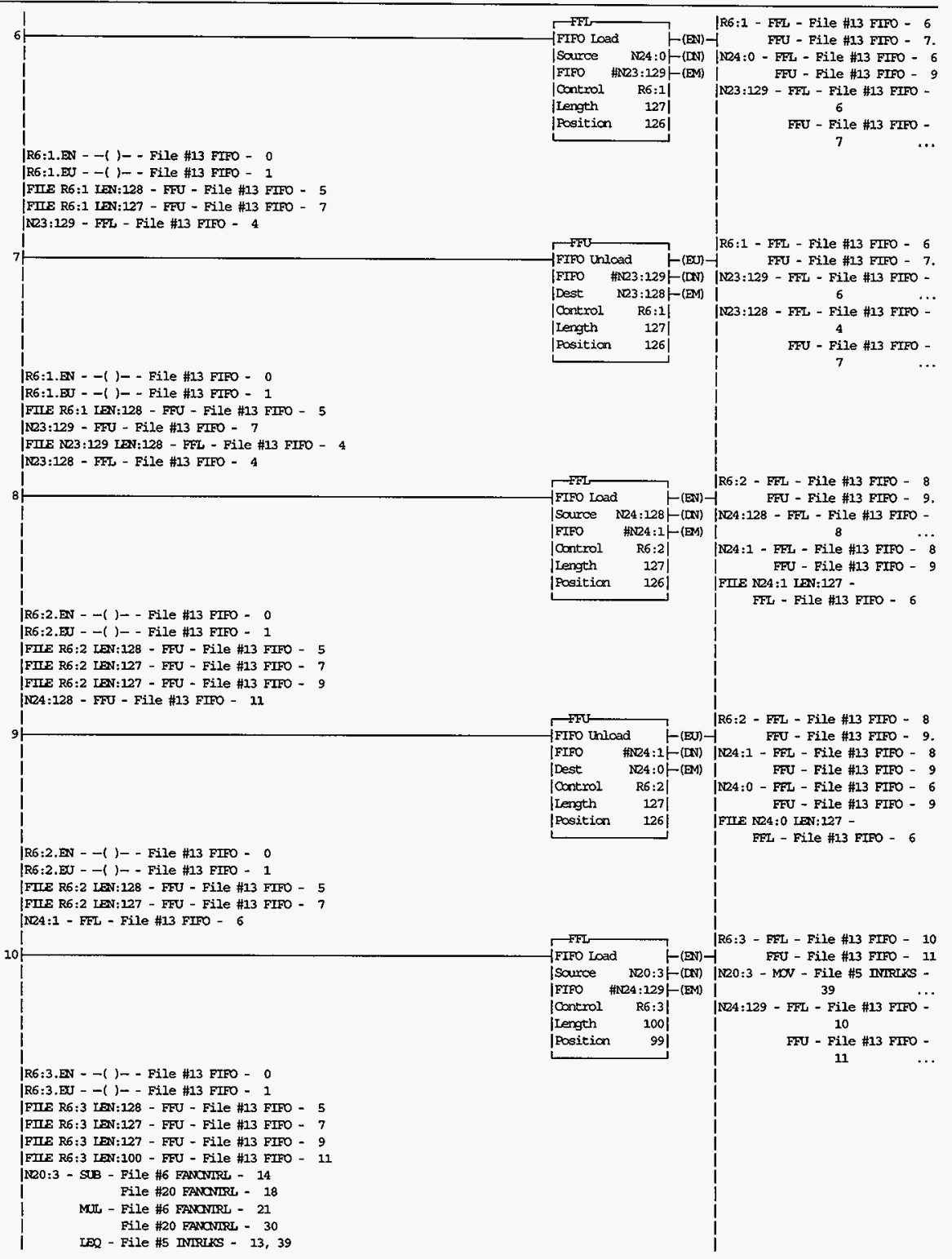




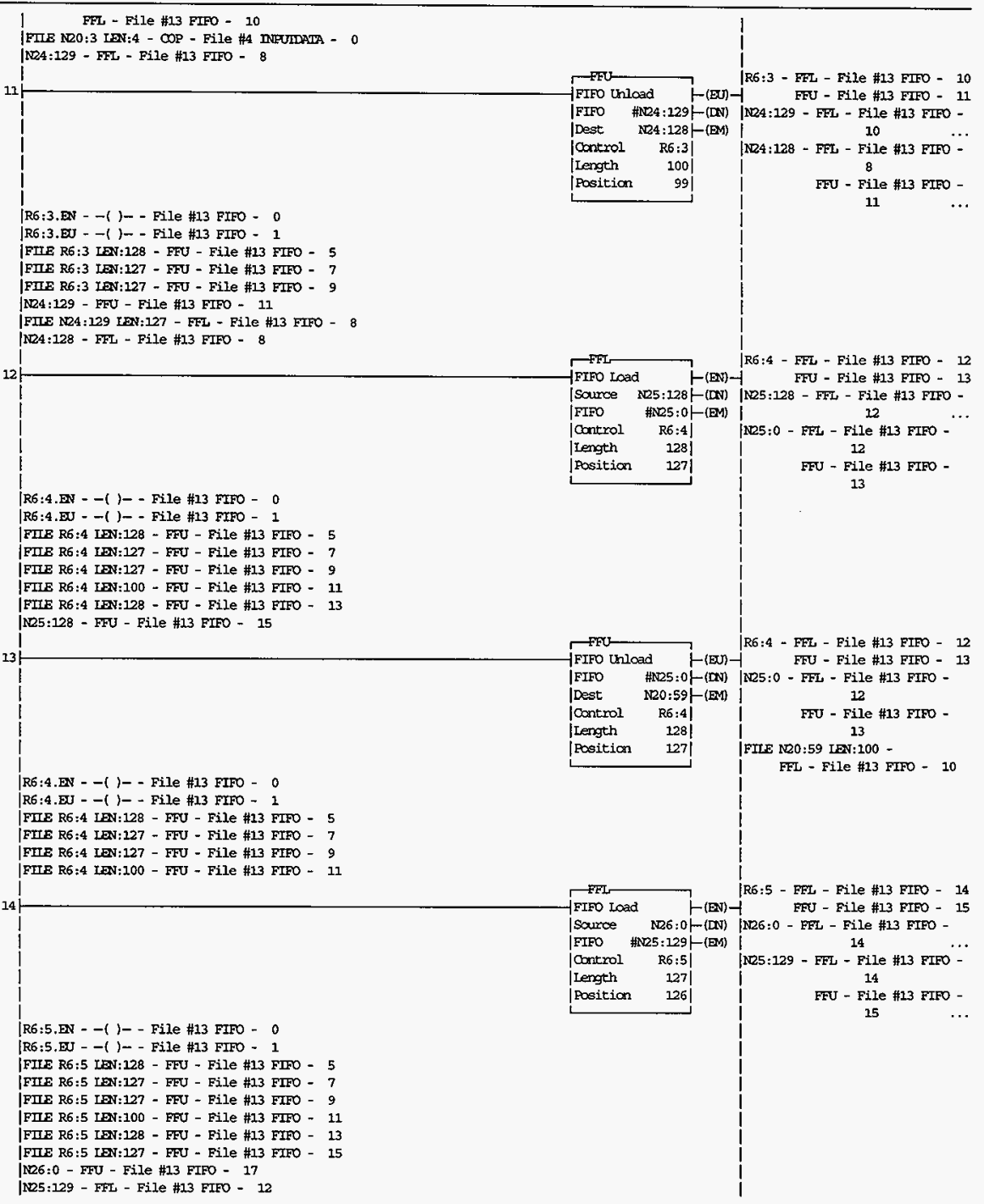




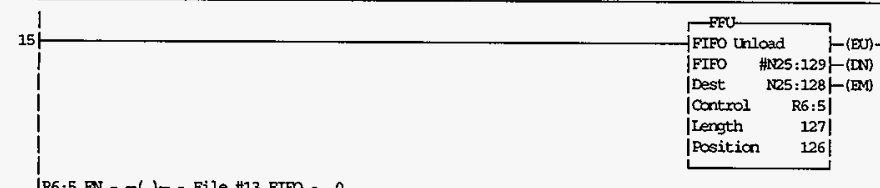

R6:5.EN - - ( )- - File \#13 FIFO - 0

|R6:5.EU - - ( ) - File \#13 FIFO - 1

|FIE R6:5 IEN:128 - FFU - File \#13 FIFO - 5

|FIIE R6:5 IEN:127 - FFU - File \#13 FIFO - 7

FIIE R6:5 IEN:127 - FFU - File \#13 FIFO - 9

FIIE R6:5 IFN:100 - FFU - File \#13 FIFO - 11

|FILE R6:5 IEN:128 - FEU - File \#13 FIFO - 13

|N25:129 - FFU - File \#13 FIFO - 15

|FIE W25:129 IEN:128 - FEL - File \#13 FIFO - 12

|N25:128 - FFL - File \$13 FIFO - 12

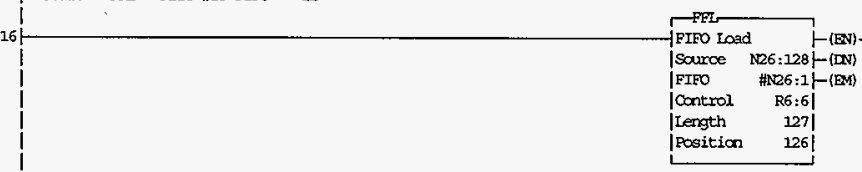

|R6:6.EN - - ( )- - File \#13 FIFO - 0

|R6:6.EI - - ( )- - File \#13 FIFO - 1

FIIE R6:6 IEN:128 - FEU - File \#13 FIFO - 5

|FIE R6:6 IDN:127 - FFU - File \#13 FIFO - 7

FIIE RS:6 IEN:127 - FFU - File \#13 FIFO - 9

|FIIE R6:6 LEN:100 - FFU - File \#13 FIFO - 11

|FUE R6:6 IEN:128 - FFU - File \#13 FIFO - 13

|FIIE R6:6 IEN:127 - FFU - File \#13 FIFO - 15

|FIIE R6:6 IEN:127 - FFU - File \#13 FIFO - 17

|N26:128 - FFU - File \#13 FIFO - 19

N26:1 - FFL - File \#13 EIFO - 14

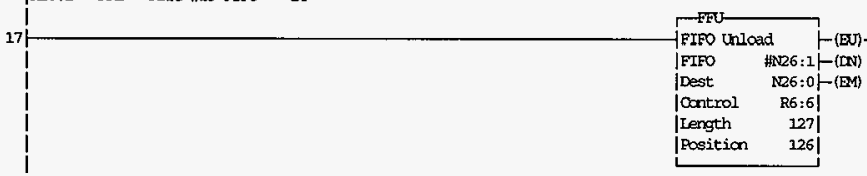

|R6:6.EN - - ( )- - File \#13 FIFO - 0

|R6:6.EU - - ( )- - File \#13 FIFO - 1

|FIIE R6:6 IPN:328 - EFU - File \#13 FIFO - 5

|FIE R6:6 IEN:127 - FFU - File \#13 FIFO - 7

|FIB R6:6 LEN:127 - FNU - File \#13 FIFO - 9

|FIIE R6:6 IEN:100 - FFU - File \#13 FIFO - 11

|FIIR R5:6 IEN:128 - FFU - File \#13 FIFO - 13

|FIIE R6:6 IEN:127 - FEU - File \#13 FIFO - 15

JN26:1 - FFU - File \#13 FIFO - 17

FII ND6:1 LDN:127 - FFL - File \#13 FIFO - 14

|N26:0 - FEL - File \#13 FIFO - 14

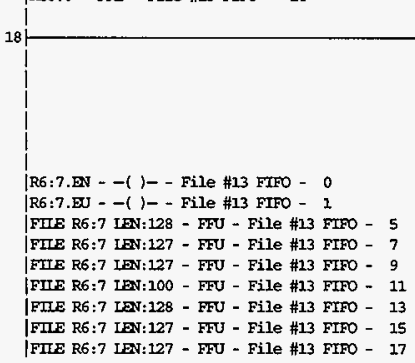

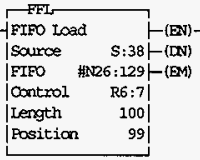

R6:7 - FFT - File \#13 FIEO - 18 FFU - File \#13 FIFO - 19 N26:129 - FFL - File \#13 FIFO FFU - File \#13 FIFO FIIE N26:129 IEN:127 -

FEL - File \#13 FIFO - 16

HNF-2934

Rev 0 


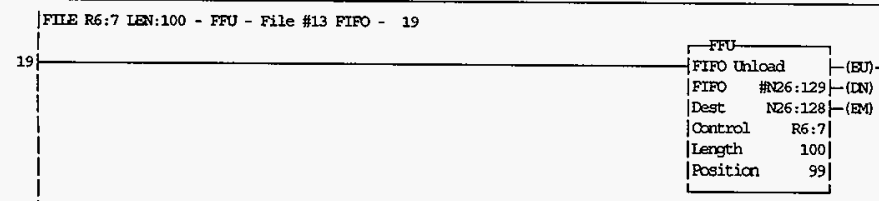

R6:7.EN - - ( )- - File \#13 FIFO - 0

R6:7.EU - - ( ) - File \#13 FIFO - 1

FIIE R6:7 LEN:128 - FFU - File \#13 FTPO - 5

|FIIE R6:7 IEN:127 - FFU - File \#13 FIFO - 7

FIIE R6:7 IDN:127 - FFU - File \#13 FIFO - 9

|FDLE R6:7 IEN:100 - FFU - File \#13 FIFO - 11

|FIE R6:7 IEN:128 - FFU - File \#13 FIFO - 13

|FIIE R6:7 IFN:127 - FFU - File \#13 FIFO - 15

|FIIE R6:7 LEN:127 - FEU - File \#13 FIFO - 17

|N26:129 - FFU - File \#13 FIFO - 19

|FIIE N26:129 IEN:127 - FFL - File \#13 FIFO - 16

N26:128 - FFL - File \#13 F1FO - 16

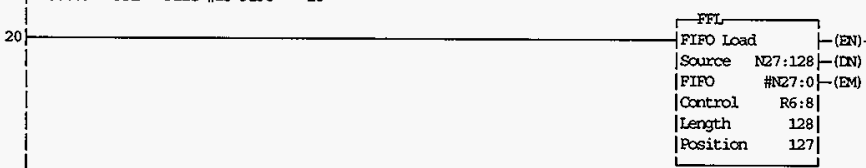

|R6:8.EN - - ( )- - File \#13 FIFO - 0

|R6:8.EU - - ( )- - File \#13 FIFO - 1

|FIIE R6:8 IEN:128 - FFU - File \#13 FIFO - 5

FIIE R6:8 IEN:127 - FWU - File \#13 FIFO - 7

|FIIE R6:8 IEN:127 - FFU - File \#13 FIFO - 9

JFIE R6:8 IEN:100 - FFU - File \#13 FIFO - 11

|FILE R6:8 IEN:128 - FFU - File \#13 FIFO - 13

|FILE R6:8 LEN:127 - FFU - File \#13 FIFO - 15

FIIE R6:8 LEN:127 - FFU - File \#13 FIFO - 17

JFIIE R6:8 IEN:100 - FFU - File \#13 FIFO - 19

|FIE R6:8 IDN:128 - FFU - FIle \#13 FIFO - 21

|N27:128 - FEU - File \#13 FIFO - 23

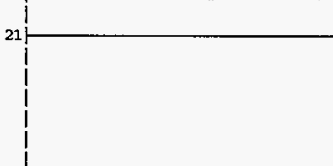

R6:8.EN - - ( ) - - File \#13 FIFO - 0

R6:8.EU - - ( ) - File \#13 FIFO - 1

FIIE R6:8 IEN:128 - FFU - File \#13 FIFO - 5

|FIIE R6:8 IEN:127 - FFU - File \#13 FIFO - 7

|FILE R6:8 IEN:127 - FFU - FIIe \#13 FIFO - 9

|FILE R6:8 IEN:100 - FFU - File \#I3 FIFO - 11

|FILE R6:8 LEN:128 - FFU - File \#13 FIFO - 13

|FTIE R6:8 IEN:127 - FFU - File \#13 FTEO - 15

FIIE R6:8 IEN:127 - FFU - File \#13 FIFO - 17

|FIIE R6:8 IEN:100 - FFU - File \#13 FIFO - 19

22

R6:9.EN - - ( ) - File \#13 FIFO -

R6:9.EU - - ( ) - File \#13 FIFO - 1

FILE R6:9 IEN:128 - FFU - File \#13 FIFO - 5

|FILE R6:9 LEN:127 - FFU - File \#13 FIFO - 7

|FILE R6:9 IFN:127 - FFU - File \#13 FIFO - 9

|FIIE R6:9 LEN:100 - FRU - File \#13 FIFO - 11

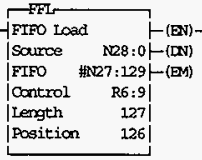

1

IR6:7 - FFL - File \#13 FIFO - 18 FFU - File \#13 FIFO - 19 |N26:129 - FFL - File \#13 FIFO N26:128 - FFL - File \#13 FIFO 16

FFU - File \#13 FIFO 19

R6:8 - FFL - File \#13 F1FO - 20 FFU - File \#13 FIFO - 21 NQ7:128 - FFL - File \#13 FIFO 20 jE7:0 - FFL - File \#13 FIFO 20 FWU - File \#13 FIFO 21
R6:9 - FFL - File \#13 FIFO - 22 FFU - File \#13 FIFO - 23 |N28:0 - FFL - File \#13 FIFO [No7:129 - FEL - File \#13 FIFO 22 FEU - File \#13 FIFO 23 
|FILE R6:9 IAN:128 - FEU - File \#13 FIFO - 13 |FIIS R6:9 IAN:127 - FFU - File \#13 FIFO - 15 |FILE R6:9 IEN:127 - FEU - File \#13 FHFO - 17 |FHE R6:9 IEN:100 - FUU - File \#13 FUFO - 19 |FIIE R6:9 LEN:128 - FFU - File \#13 FTFO - 21 |FIIB R6:9 LEN:127 - FFU - File \#13 FIFO - 23 |N28:0 - FWU - File \#13 EIFO - 25 |N27:129 - FFL - File \#13 FIFO - 20

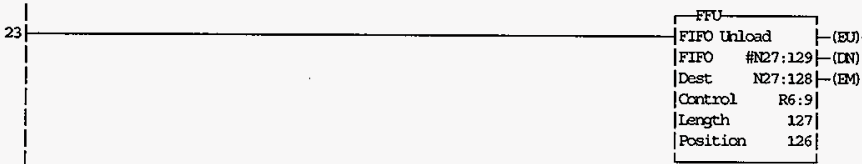

126:9.EN - -( )- - File \#13 FIFO - 0 |R6:9.EU - - ( ) - - File \#13 FIFO - 1 |FIIE R6:9 ERT:128 - FFU - File \#13 FIFO - 5 |FILE R6:9 EDS:127 - FFU - File \$13 FIFO - 7 |FIE R6:9 LEN:127 - FFU - File \#13 FIFO - 9 |FIIE R6:9 LEN:100 - FFU - File \#13 FIFO - 11 |FIE R6:9 IEN:128 - FFU - File \#13 FIFO - 13 |FIIE R6:9 LEN:127 - FFU - File \#13 FIFO - 15 |FITE R6:9 IDN:127 - FFU - File \#13 FIFO - 17 |FIIE R6:9 LEN:100 - FFU - File \#13 FIFO - 19 |FIE R6:9 EDN:128 - FFU - File \#13 FIFO - 21 |N27:129 - FFU - File \#13 FIFO - 23 |FIIE N27:129 IEN:128 - FFI, - File \#13 FTFO - 20 |N27:128 - FFL - File \#13 FIFO - 20

$$
24
$$

|R6:10 - FFU - File \#13 FIFO - 25

RE:10.EN - - ( ) - File \#13 FIFO - 0

|R6:10.EU - - ( ) - File \#13 FFFO - 1

|FIIE R6:10 IEN:128 - FFU - File \#13 FIFO - 5 |FIE R6:10 IEN:127 - FFU - File \#13 FIFO - 7 |FITE R6:10 IEN:127 - FFU - File \#13 FIFO - 9 |FIIE R6:10 IEN:100 - FEU - File \#13 FIFO - 11 FHE R6:10 IEN:128 - FFU - File \#13 FIFO - 13 |FIE R6:10 IEN:127 - FFU - File \$13 FIFO - 15 FHE R6:10 IEN:127 - FFU - File \#13 FIFO - 17 FIIE R6:10 IEN:100 - FFU - File \#13 FIFO - 19 |FIIE R6:10 IDN:128 - FFU - File \#13 FIFO - 21 FIIE R6:10 IBV:127 - FFU - File \#13 FIFO - 23 |FIIE R6:10 INN:127 - FFU - File \#13 FIFO - 25 WD2 :128 - FFU - File \#13 FIFO - 27 N28:1 - FFL - File \#13 FIFO - 22

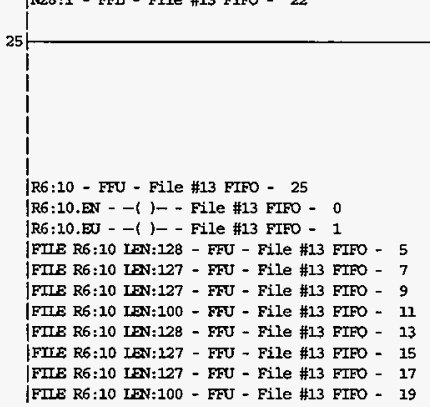

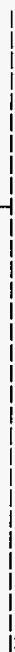

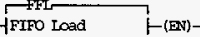
|FIFO \#NE8:1-(EM) Control R6:30| ILength 127 |Position 126 |

R6:9 - FFL - File \#13 FIFO - 22 FFU - File \#13 FIFO - 23 |N27:129 - FFL - File \#13 FIFO NR7:128 - FFL - File \#13 FIFO FFU - ELIe \#13 FIFO 23

R6:10 - FFL - File \#13 FIFO N28:128 - FFL - File \#13 FIEO NE8:1 - FFL - File \$13 FIFO FFU - File \#13 FIFO 25

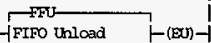
FTFO |Dest NQ8:0|-(BM) |Control R6:10| |Length 127| |Pasition 126| 
|FIIE R6:10 IAN:128 - FWU - File \#13 FIFO - 21 FIIE R6:10 IDN:127 - FEU - File \#13 FTFO - 23 |N28:1 - FFU - File \#13 FIFO - 25

|FIIE N28:1 IDN:127 - FFL - File \#13 FIFO - 22 |N28:0 - FFL - File \#13 FIFO - 22

$$
\mid
$$

R6:11 - FFU - File \#13 FIFO - 27

R6:11.EN - - ( ) - File \#13 FIFO - 0

R6:11.EJ - - ( ) - File \#13 FIFO - 1

|FIIE R6:11 IEN:128 - FFU - File \#13 FIFO - 5

FIIE R6:11 IEN:127 - FFU - File \#13 FIFO - 7 |FIIE R6:11 IEN:127 - FFU - File \#13 FTFO - 9 |FIE R6:11 IEN:100 - FFU - File \#13 FTFO - 11 |FIE R6:11 IDN:128 - FFU - File \#13 EIFO - 13 FIIE R6:11 LEN:127 - FPU - File \#13 FIFO - 15 |FIIE R6:11 IEN:127 - FU - File \#13 FIFO - 17 |FIEE R6:11 IEN:100 - FFU - File \#13 FTFO - 19 FTIE R6:11 IFN:128 - FFU - File \#13 FTFO - 21 |FIIE R6:11 IEN:127 - FFU - File \#13 FIFO - 23 FIIE R6:II IEN:127 - FNU - File \#13 FIFO - 25 |FIIE R6:11 IEN:100 - FRU - File \#13 FIFO - 27 IN28:129 - FFL - File \#13 FIFO - 24$$
\text { 27 }
$$

R6:11 - FFU - File \#13 FIFO - 27

|R6:11.EN - - ( ) - File \#13 FIFO - 0

R6:11.E0 - - ( ) - File \#13 FIFO - 1

|FIIE R6:11 IEN:128 - FFU - File \#13 FIFO - 5

FIIE R6:I1 IEN:127 - FFU - File \#13 FIFO - 7

|FIIE R6:11 LEN:127 - FFU - File \#13 FTFO - 9

FTIE R6:11 IEN:100 - FFU - File \#13 FIFO - 11

|FTIE R6:11 IEN:128 - FNU - File \#13 FIPO - 13

FIIE R6:11 IEN:127 - FRU - File \#13 FIPO - 15

|FIE R6:11 IEN:127 - FFU - File \#13 FIPO - 17

FIIE R6:11 IDN:100 - FEU - FIle \#13 FIFO - 19

|FHE R6:11 IEN:128 - FFU - File \#13 FIFO - 21

|FIIB R6:11 LEN:127 - FFU - File \#13 FIFO - 23

FTLE R6:11 IFN:127 - FFU - File \#13 FIFO - 25

N28:129 - FFU - File \#13 FIFO - 27

FIIE N28:129 LEN:127 - FFL - File \#13 FIFO - 24

|N28:128 - FFL - File \#13 FIFO - 24

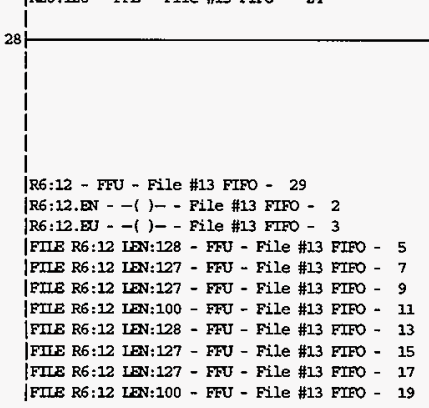

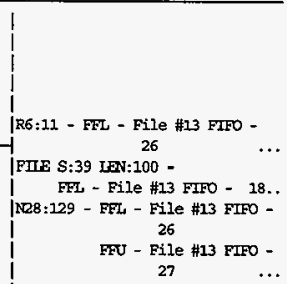

FIFO Ioad

sounce

$\mathrm{S}: 39-(\mathrm{CN})$ |FIFO |Control R6:11| |Length $\quad 100 \mid$ |Fosition 99|

$$
27
$$

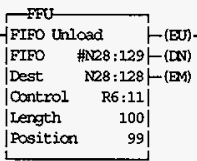

RR6:11 - FEL - File \#13 FIFO -
HN2:129 - FFL - File \#13 FIFO - $26 \quad \ldots$ NE28:128 - FFL - File \#13 FIFO - 24
FWU - File \#13 FIFO - 27

R6:12 - FFL - File \#13 FIFO FIFO Laad Source N29:128 (DN) N29:128 - FFL - File \#13 FIFO |FIFO \#R29:01-(BM) Control R6:12 $\begin{array}{ll}\text { |Length } & 128 \text { | } \\ \text { position } & 127 \text { | }\end{array}$ INE9:0 - FFL - File \#13 FIFO 28 FFU - File \#13 FIFO 29 
|FIIE R6:12 LEN:128 - FUU - File \#13 FIFO - 21 |FIIE R6:12 IDN:127 - FFU - File \#13 FTFO - 23 |FIIE R6:12 IDN:127 - FFU - File \#13 FIFO - 25 |FIIE R6:12 IEN:100 - FFU - File \#13 FIFO - 27 |FIIE R6:12 IAN:128 - FEU - File \#13 FIFO - 29 |N29:128 - FFU - File \#13 FIFO - 31

29

I

1

I

|R6:12 - FFU - File \#13 FIFO - 29

R6:12.EN - - ( ) - - File \#13 FIFO - 2

R6:12.EU - - ( ) - File \#13 FIFO - 3

|FIE R6:12 LEN:128 - FFU - File \#13 FIFO - 5

|FIIE R6:12 LEN:127 - FFU - File \#13 FIFO - 7

|FIIB R6:12 IEN:127 - FEU - File \#13 FIFO - 9

|FII R6:12 IEN:100 - EFU - Eile \#13 FIFO - 11

|FIIE R6:12 IEN:128 - FFU - File \#13 FIFO - 13

FIIE R6:12 IEN:127 - FFU - File \#13 FIFO - 15

|FIIE R6:12 LEN:127 - FFU - File \#13 FIFO - 17

|FIIE R6:12 LEN:100 - FFU - File \#13 FTFO - 19

|FIEE R6:12 LEN:128 - FFU - Pile \#13 FIFO - 21

|FIIE R6:12 IEN:127 - EFU - Eile \#13 FIFO - 23

|FIIE R6:12 IDN:127 - FFU - File \#13 FIFO - 25

|FIIE R6:12 IEN:100 - FFU - File \#13 FIFO - 27

30

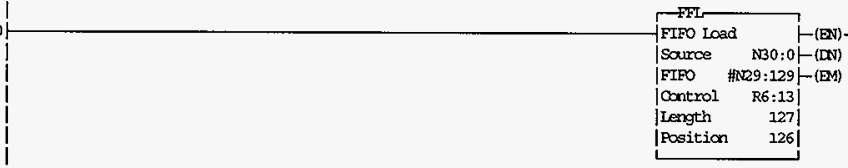

|R6:13 - FFU - File \#13 FIFO - 31

|R6:13. BN - - ( ) - - File \#13 FIFO - 2

|R6:13.BU - -( )- - File \#13 FIFO - 3

|FIIE R6:13 LEN:12B - FIU - File \#13 FIFO - 5

|FIIE R6:13 IDN:127 - FFU - File \#13 FIFO - 7

|FIIE R6:13 LEN:127 - FFU - File \#13 FIFO - 9

|FIIE R6:13 LEN:100 - FFU - File \#13 FIFO - 11

|FIIE R6:13 IFN:128 - FFU - File \#13 FIFO - 13

|FILE R6:13 IEN:127 - FFU - File \#13 FIFO - 15

|FIIE R6:13 LEN:127 - FHU - File H13 FIFO - 17

|FITE R6:13 LDN:100 - FFU - File \#13 FIFO - 19

|FIIE R6:13 LDN:128 - FRU - File \#13 FIFO - 21

|FIIE R6:13 LEN:127 - FFU - File \#13 FIFO - 23

|FIIE R6:13 LEN:127 - FFU - File \#13 FIFO - 25

|FIIE R6:13 IEN:100 - FFU - File \#13 FIFO - 27

|FIE R6:13 IEN:128 - FWU - File \#13 FIFO - 29

|FILE R6:13 LAN:127 - FFU - File \#13 FIFO - 31

|N30:0 - FFU - File \#13 FIFO - 33

IN29:129 - FFL - File \#13 FIFO - 28

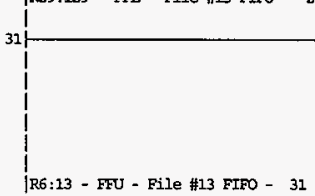

|R6:13.EN - - ( )- - File \#13 FIFO - 2

|R6:I3.EU - - ) - File \#13 FIFO - 3

FILE R6:13 LEN:128 - FFU - File \#13 FIFO - 5

|FIIE R6:13 LEN:127 - FFU - File \#13 FIFO - 7

|FIIE R6:13 IFN:127 - FWU - File \#13 FIFO - 9

|FILE R6:13 LEN:100 - FFU - File \#13 FIRO - 11

|FIIE R6:13 LON:128 - FNU - File \#13 FIFO - 13

|FIE R6:13 LEN:127 - FFU - File \#13 FIFO - 15

(1)

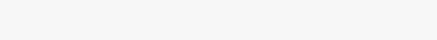

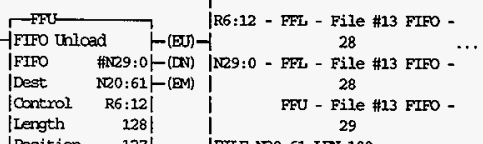

|Position 127|

|FIIE N20:61 IEN:100 -

FFL - File \#13 FIFO - 10
1
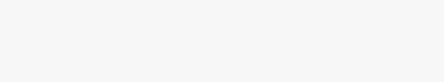

R6:13 - FFL - File \#13 FIFO 30 NB30:0 - FEL - File \#13 FIFO N29:129 - FFL - File \#13 FTFO 30 FFU - File \#13 FIFO 31 

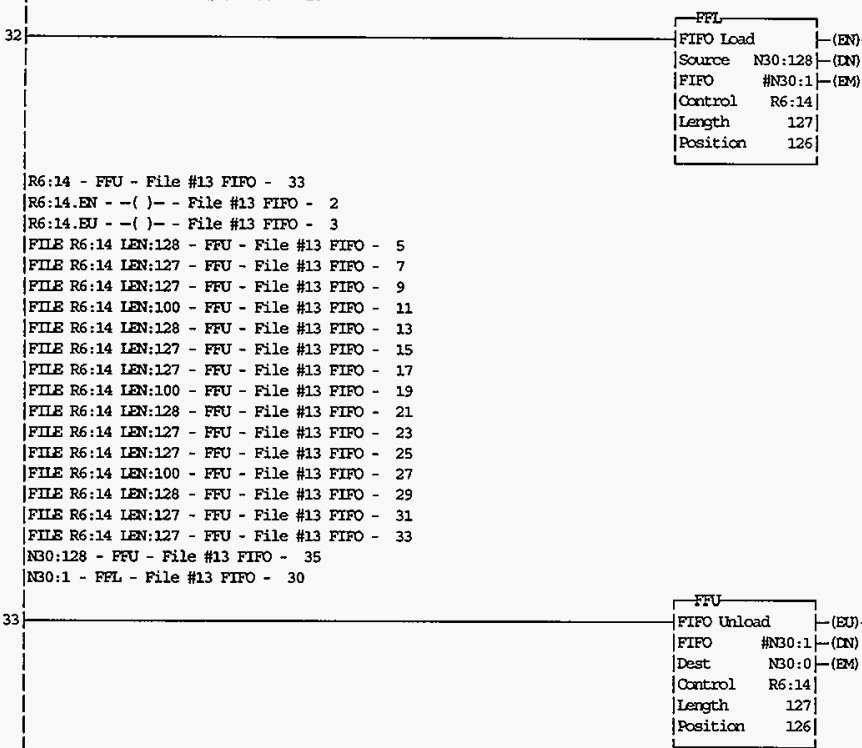

|FIE R6:14 IEN:128 - FFU - Eile \#13 FIFO - 13

|FIIE R6:14 IEN:127 - FFU - EIIE \#13 FIFO - 15

|FIIE R6:14 IEN:127 - FFU - File \#13 FIFO - 17

|FIIE R6:14 LEN:100 - FFU - File \#13 FIFO - 19

|FIIE R6:14 IEN:128 - FFU - File \#13 FIFO - 21

FIIE R6:14 IEN:127 - FFU - File \#13 FIPO - 23

|FIIE R6:14 IEN:127 - FFU - File \#13 FIFO - 25

FIIE R6:14 IEN:100 - FFU - File \#13 FIFO - 27

FIIE R6:14 IEN:128 - FFU - File \#13 FIFO - 29

FIIE R6:14 IEN:127 - FFU - File \#13 FIFO - 31

NB0:1 - FEU - File \#13 FIFO - 33

|FLE NBO:1 IEN:127 - FFL - File \#13 FIFO - 30

1NB0:0 - FFL - File \#13 FIFO - 30

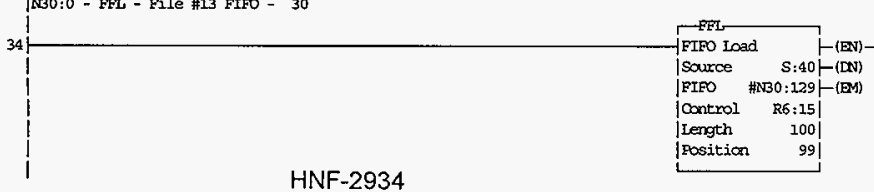

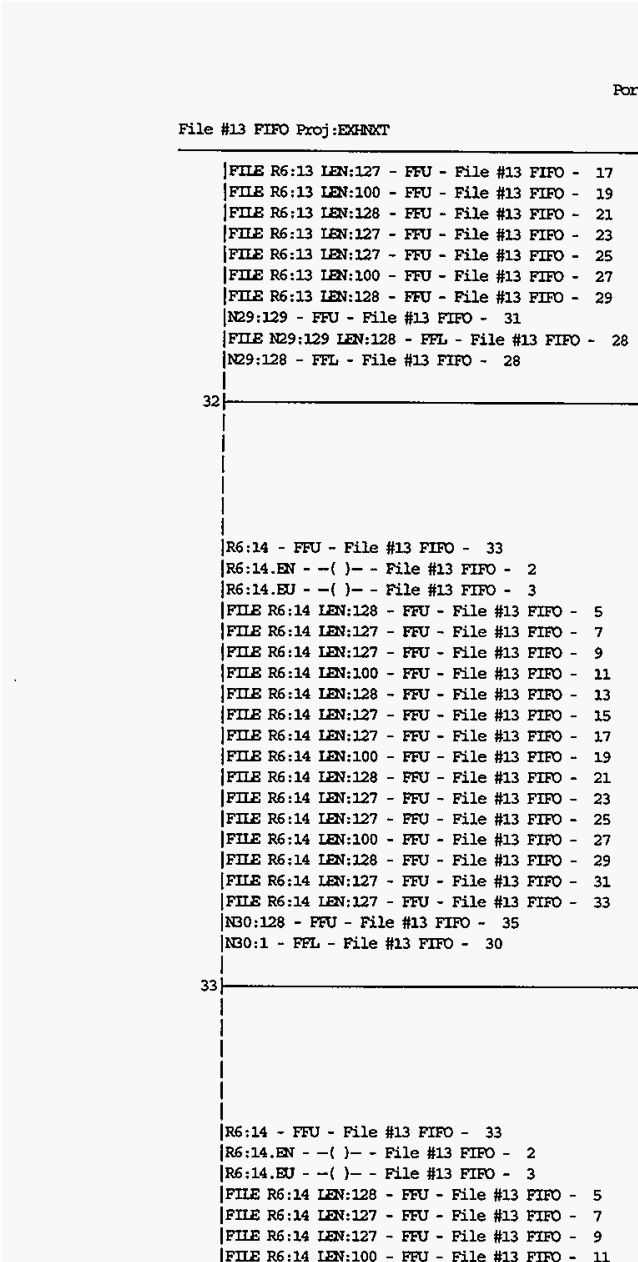

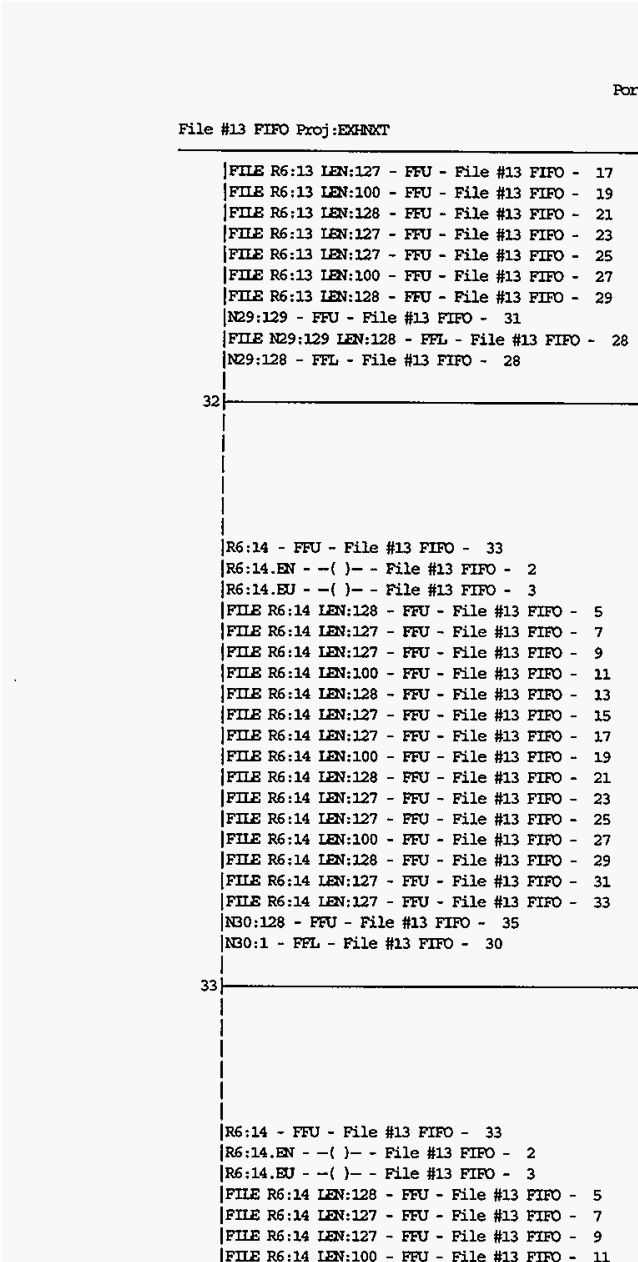

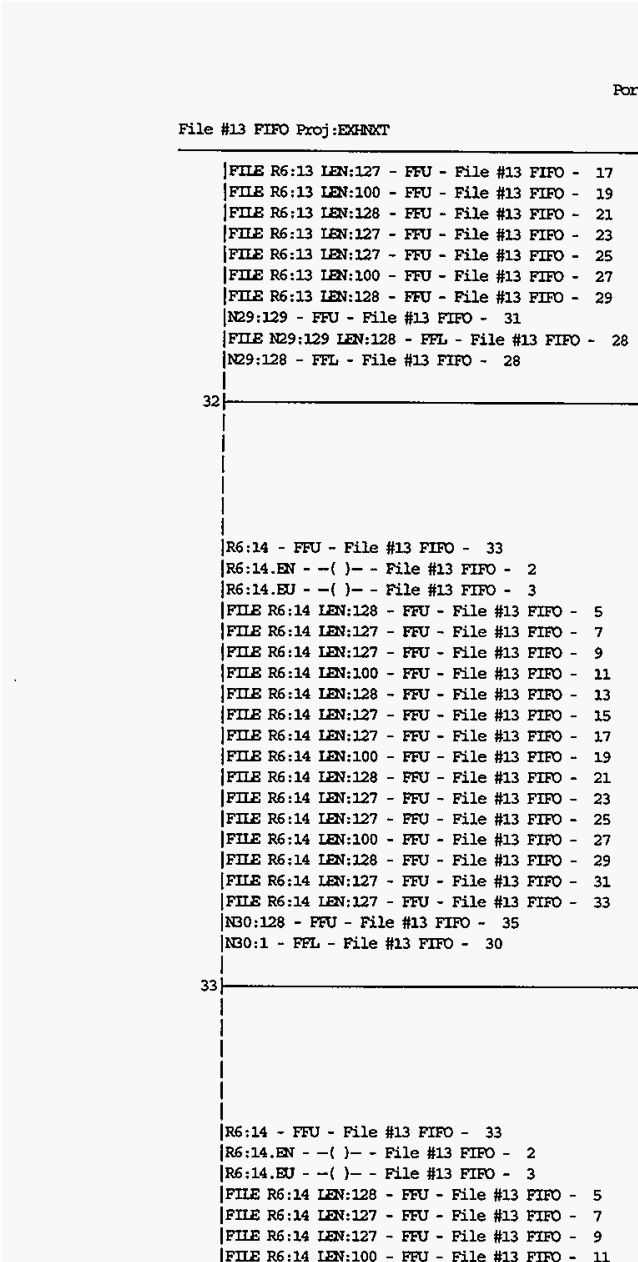

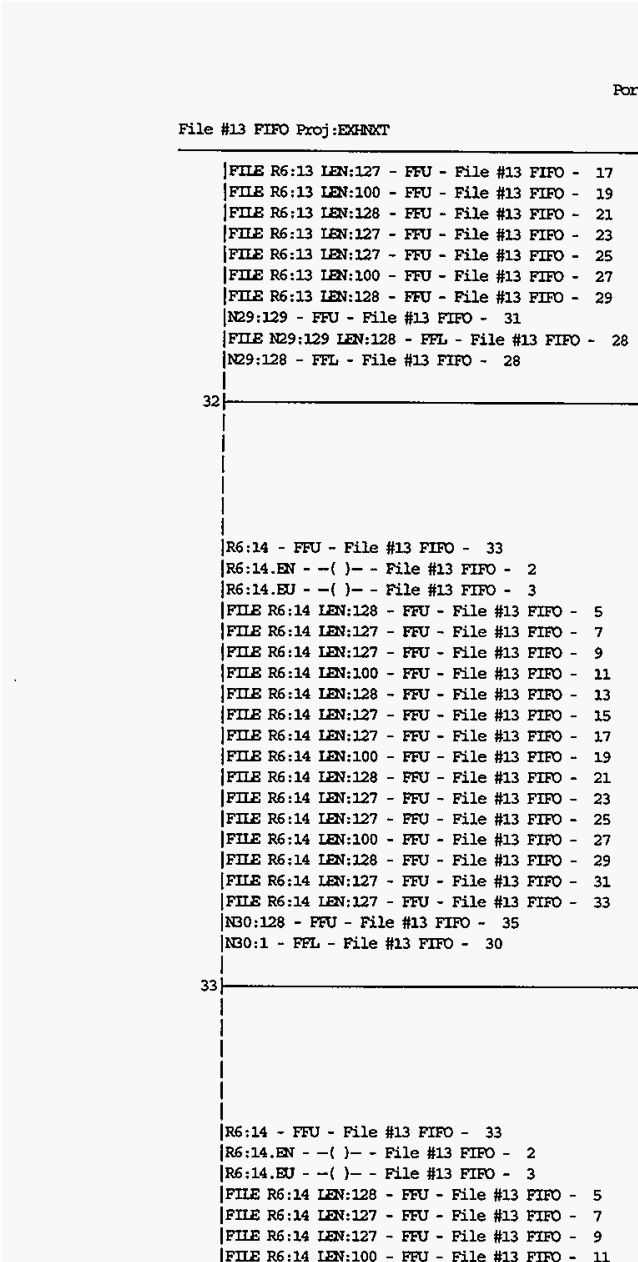

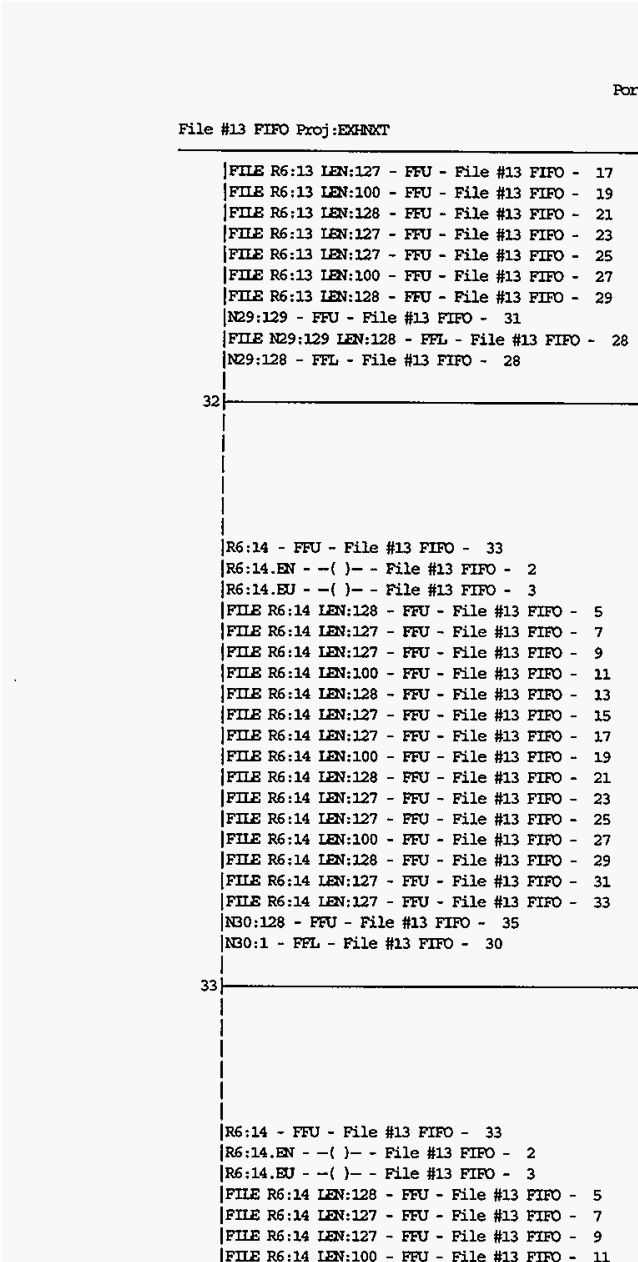

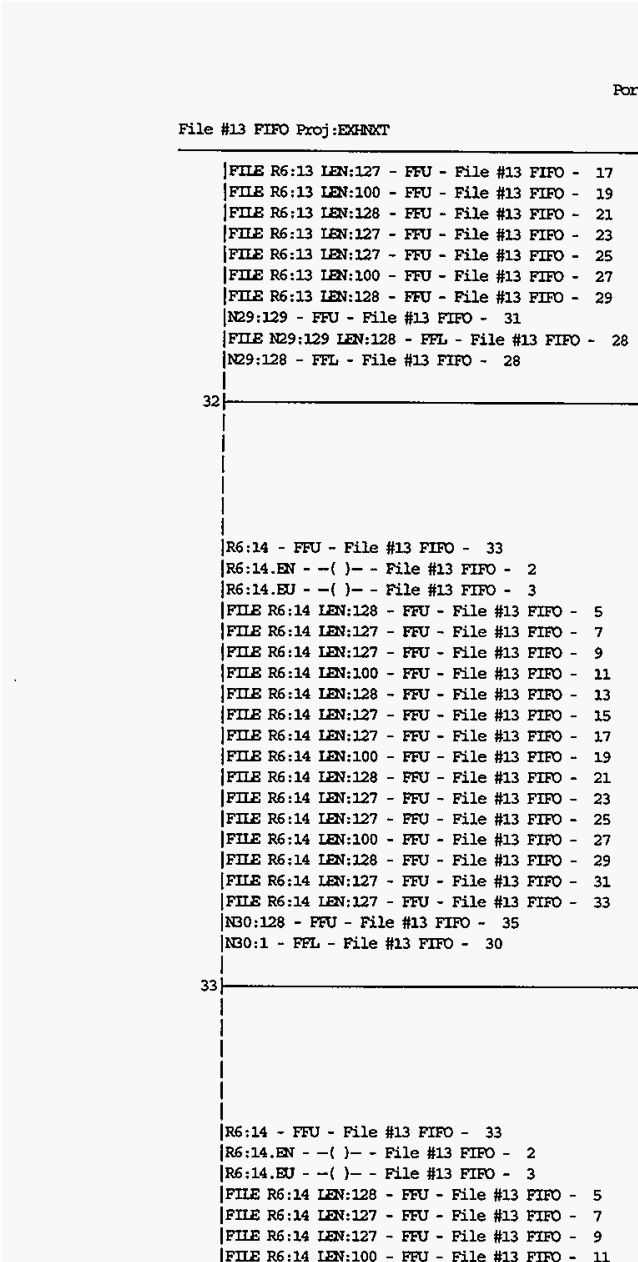

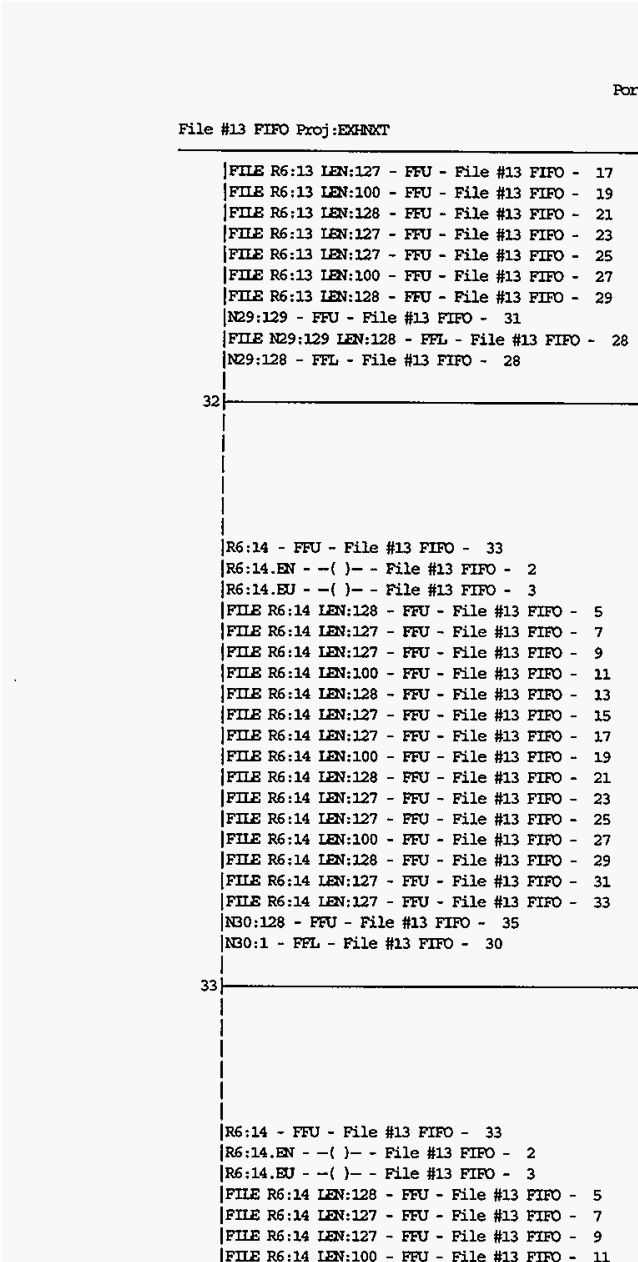

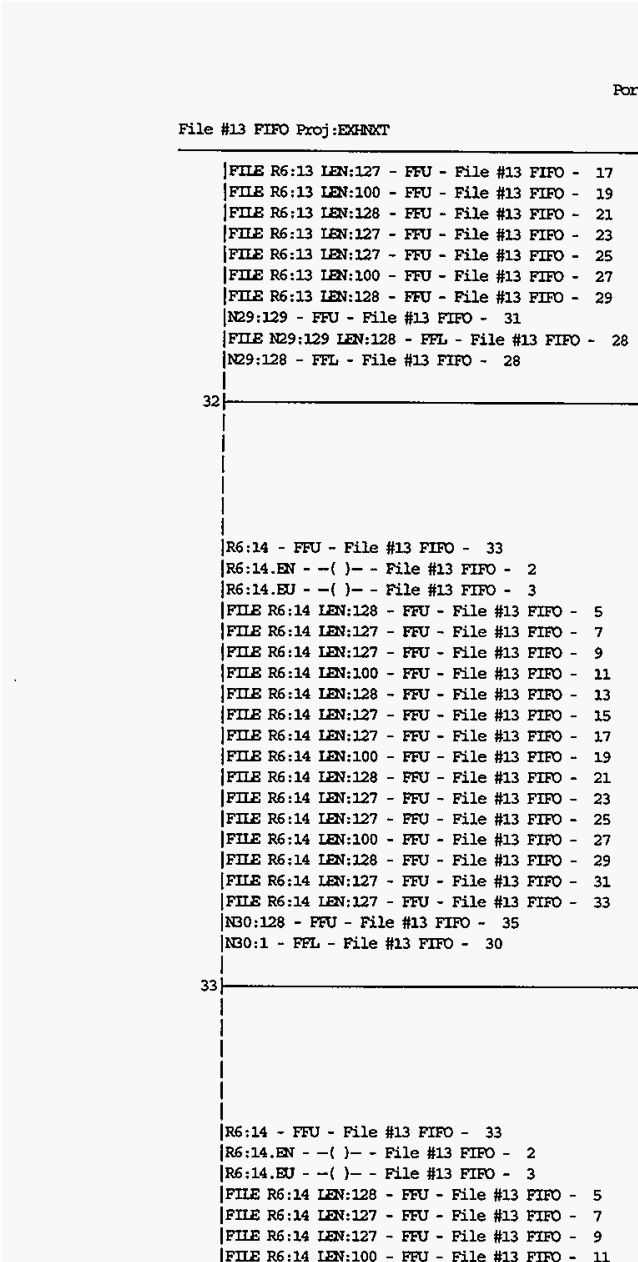

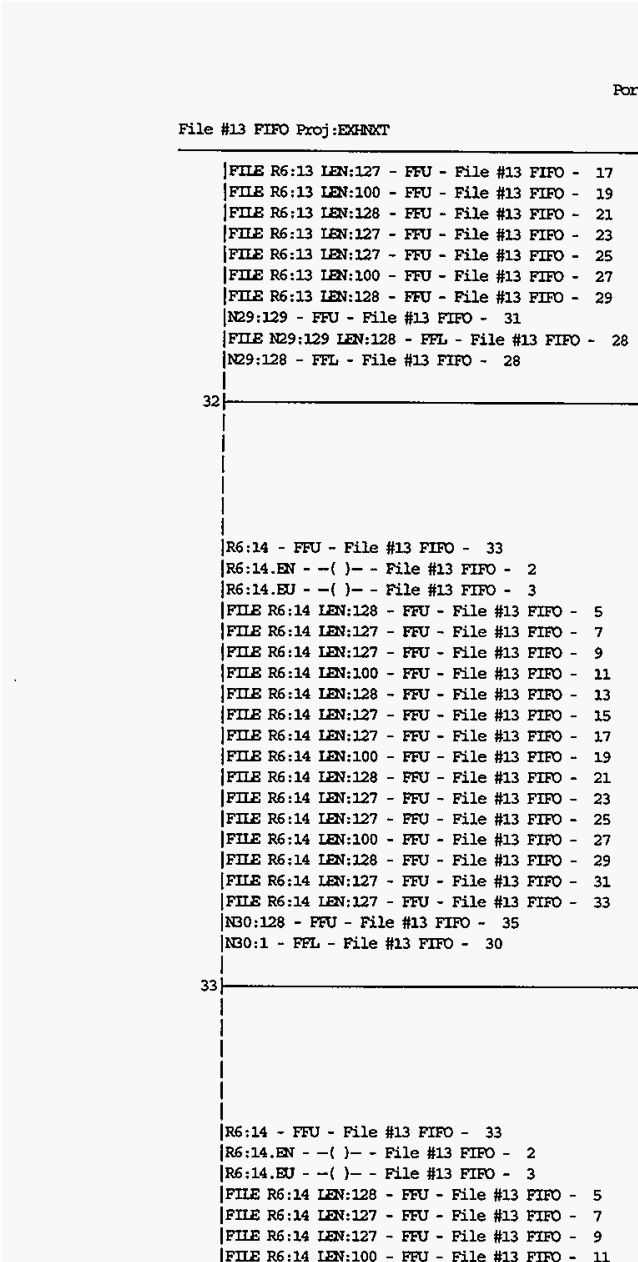

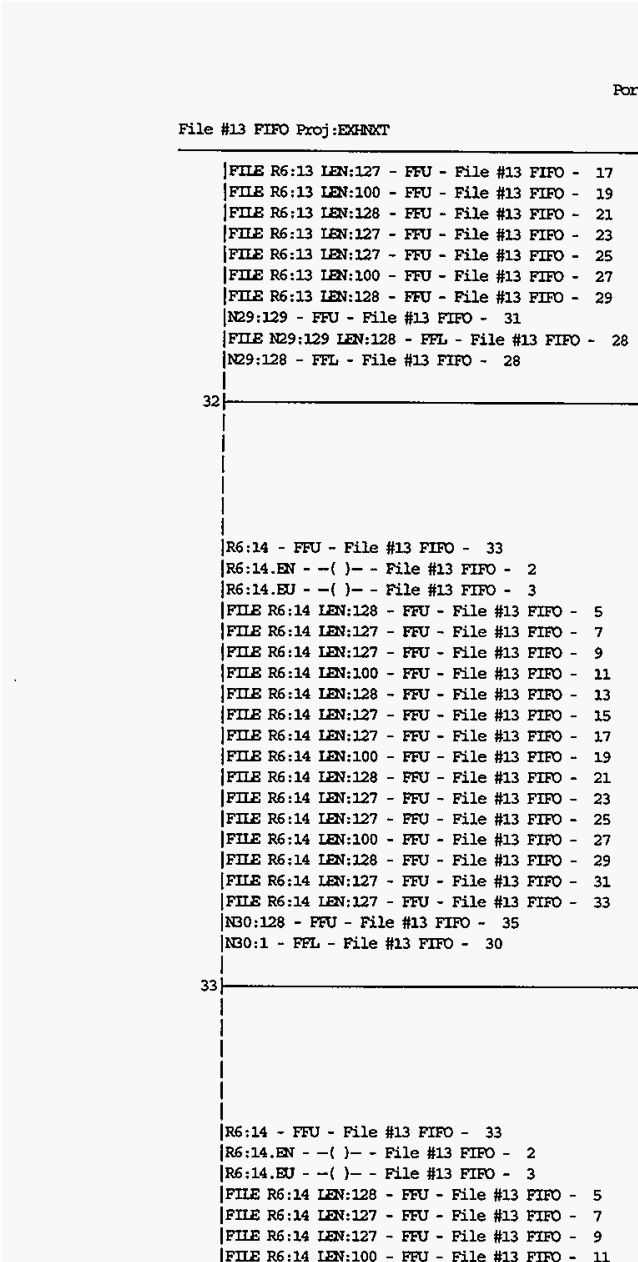

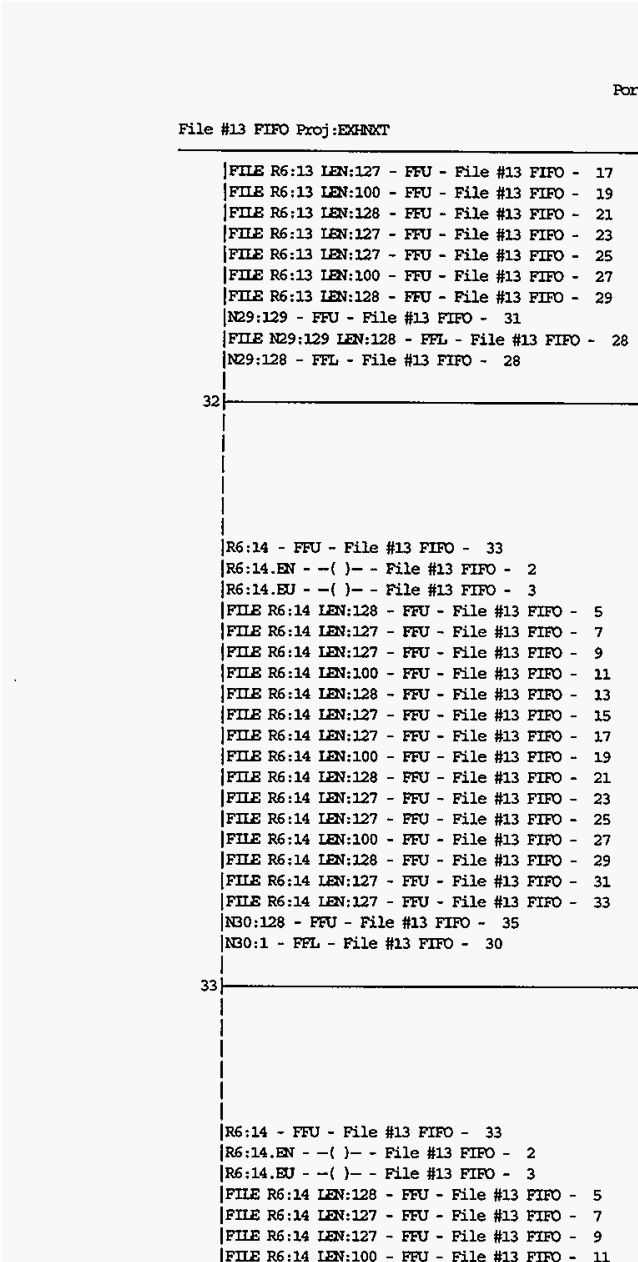

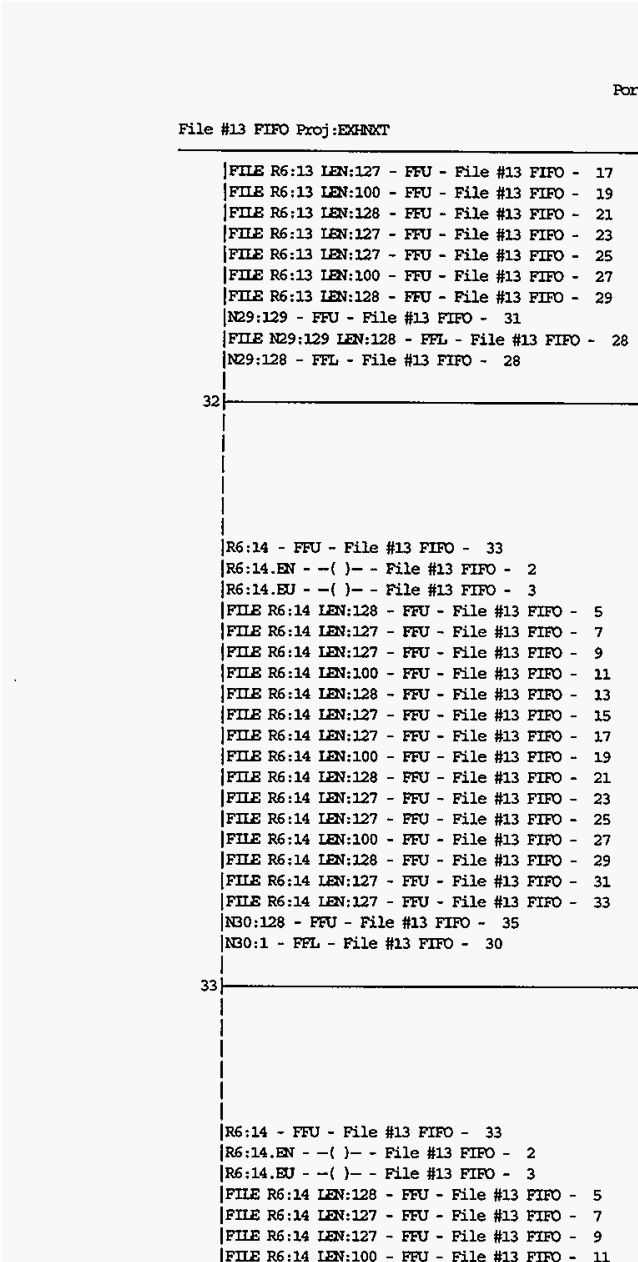

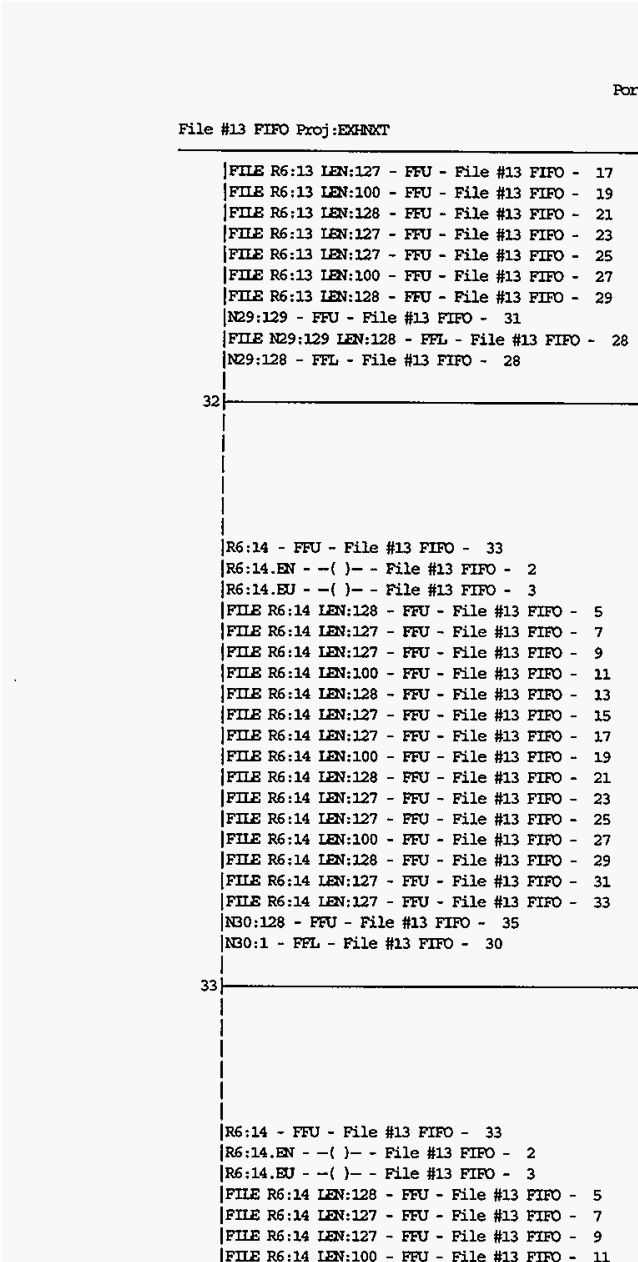

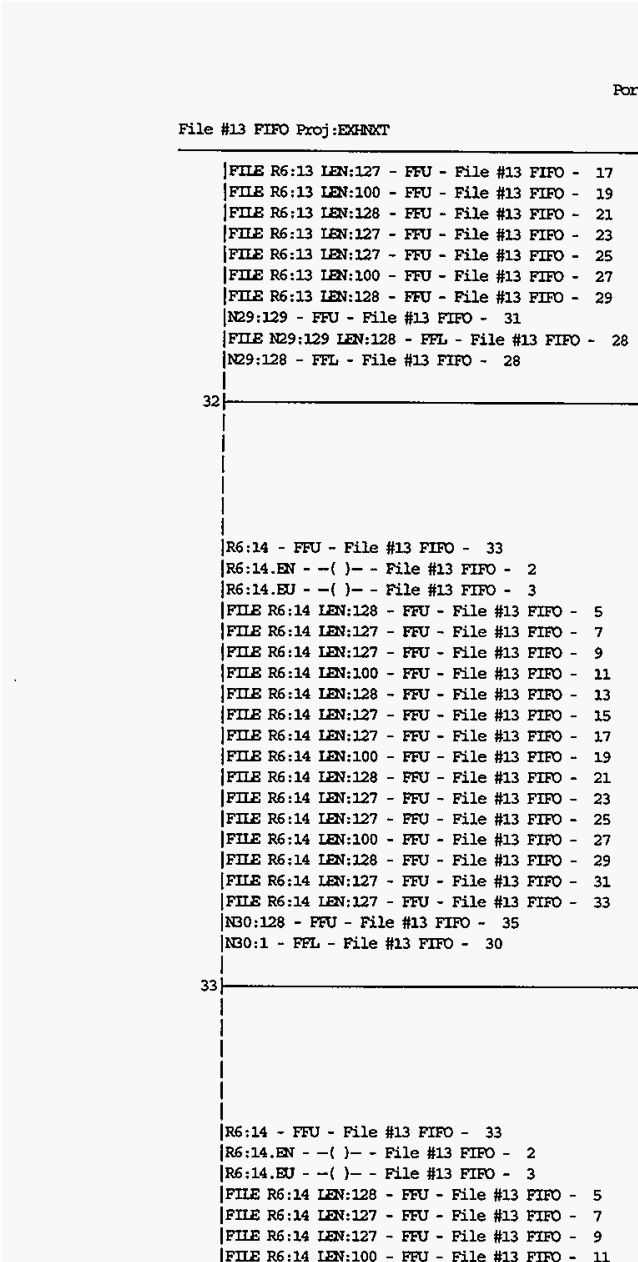

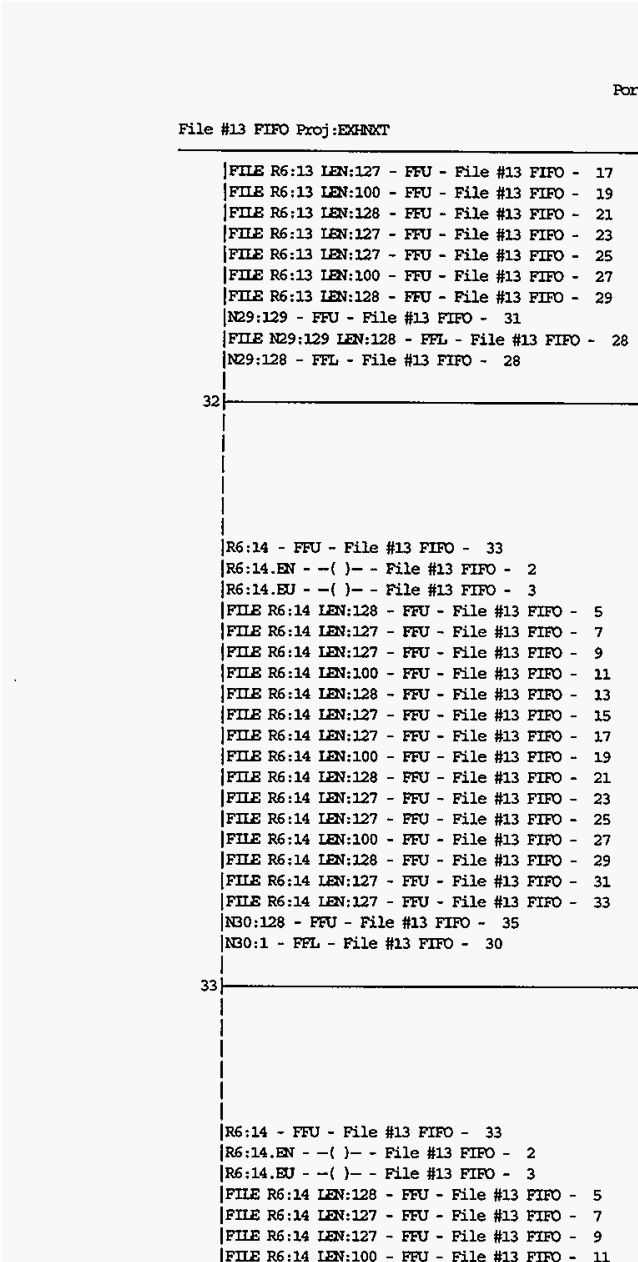

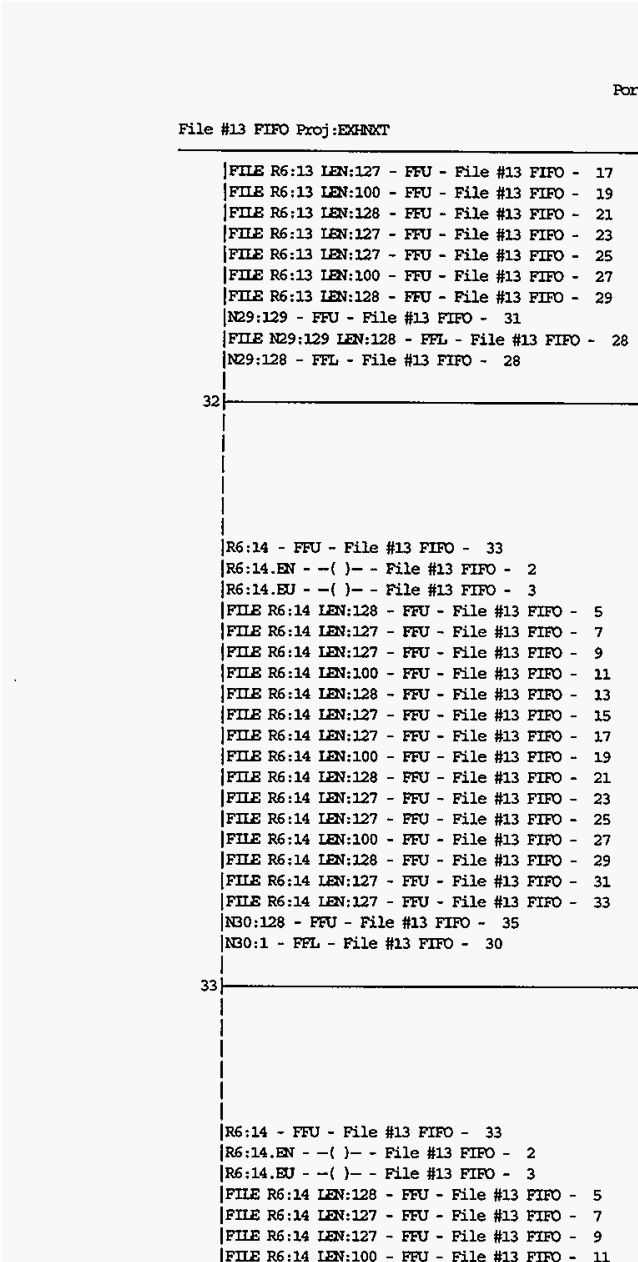

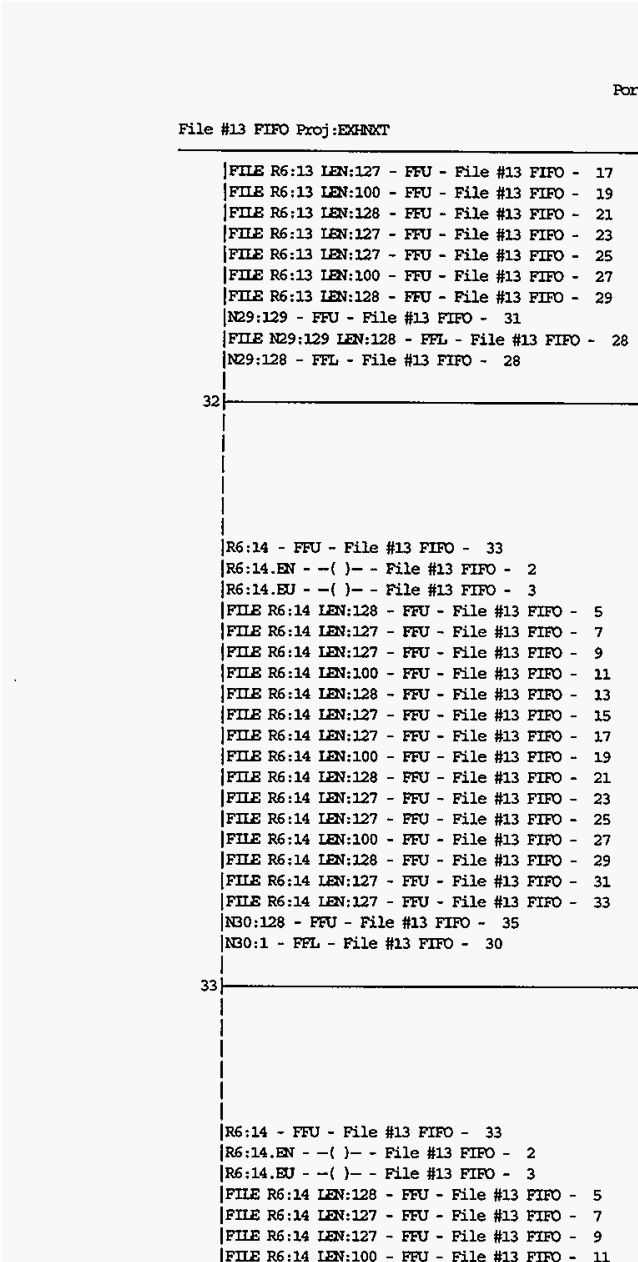

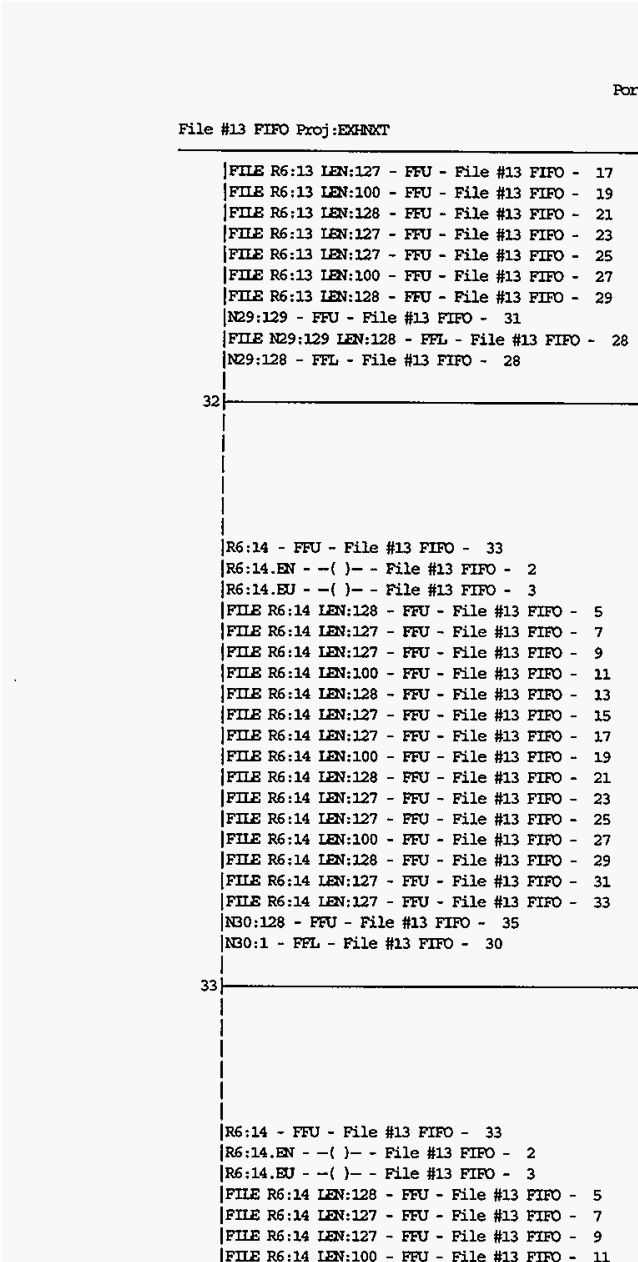

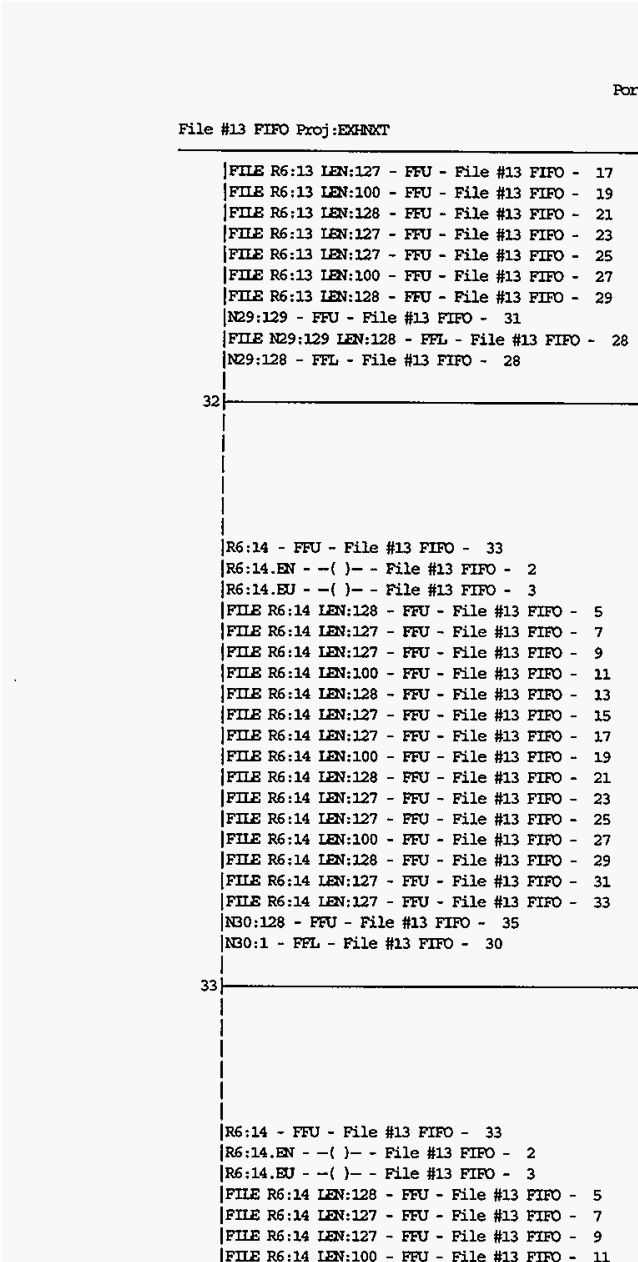

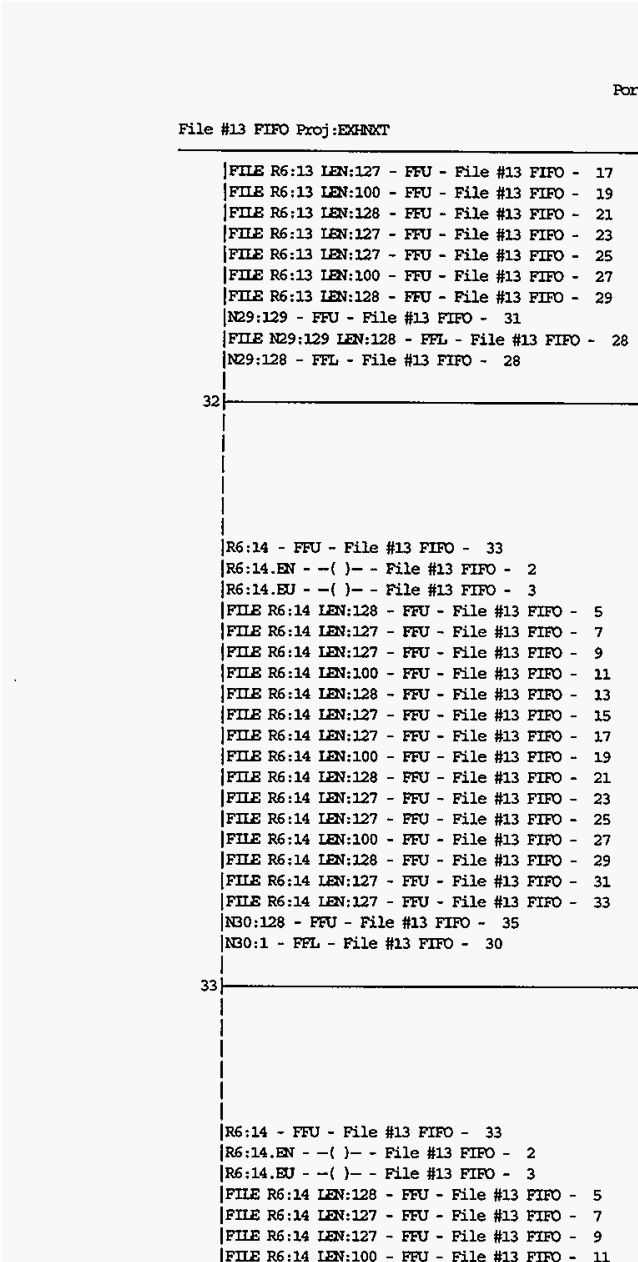

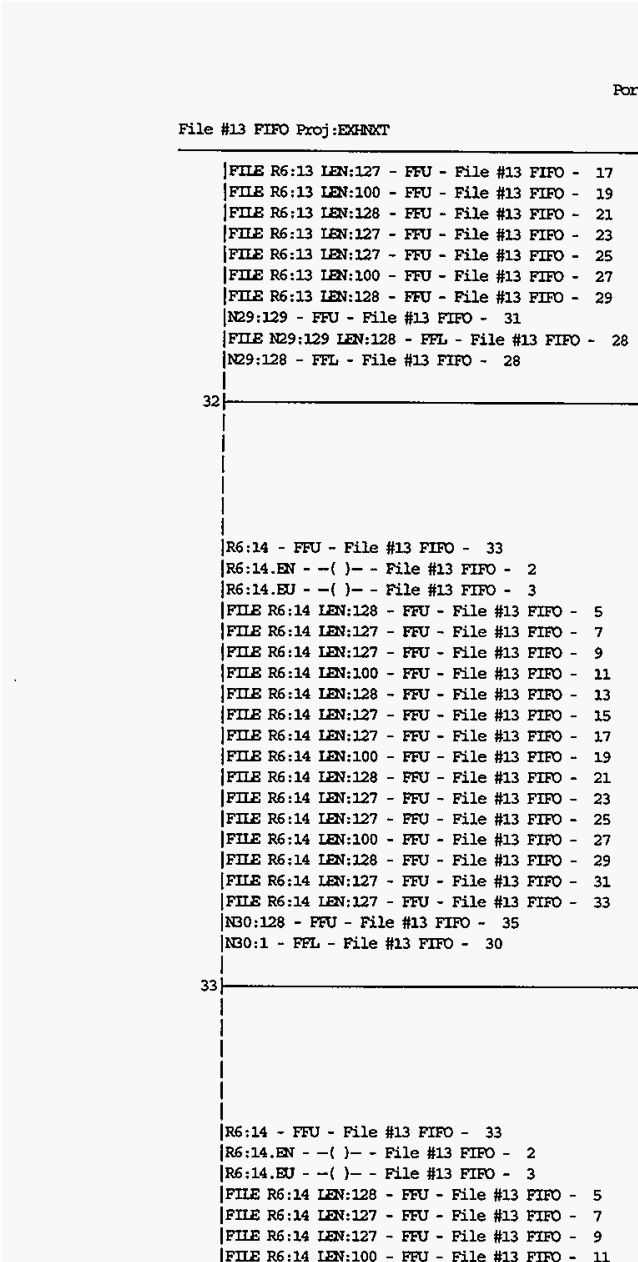

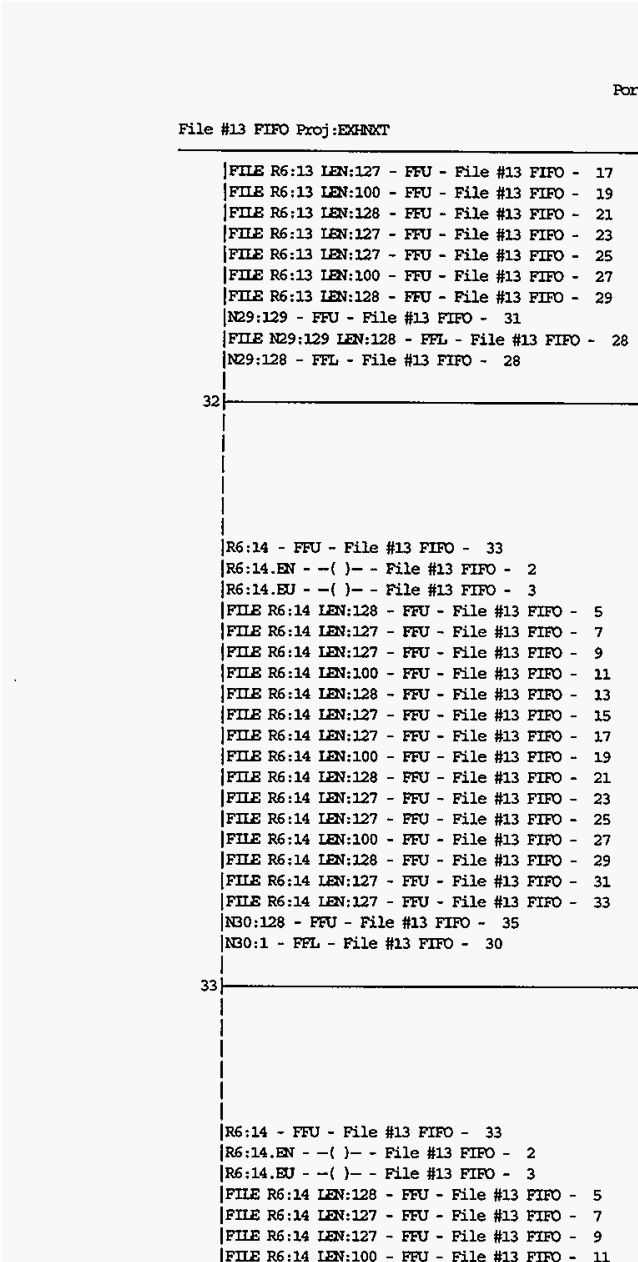

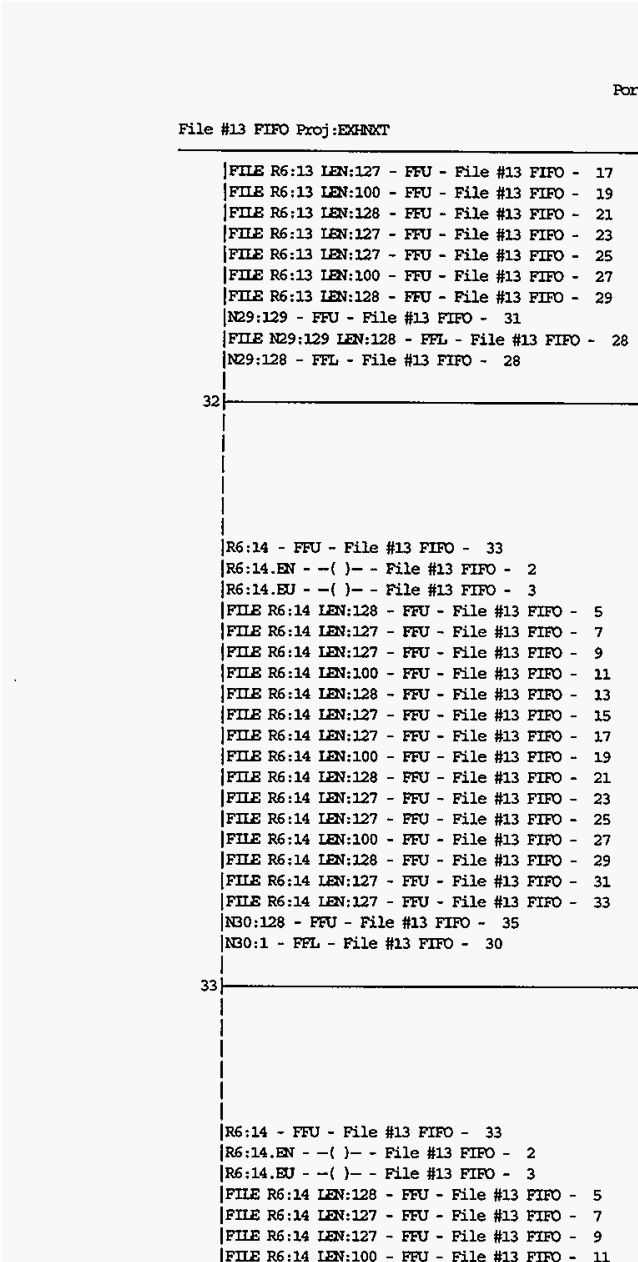

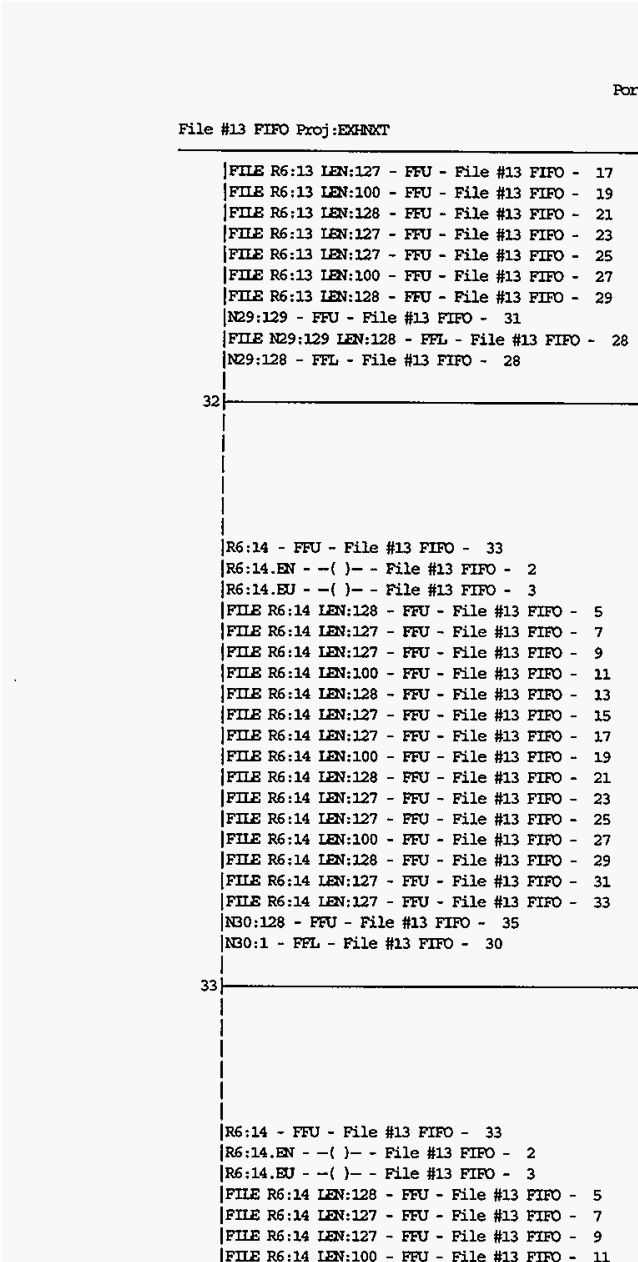

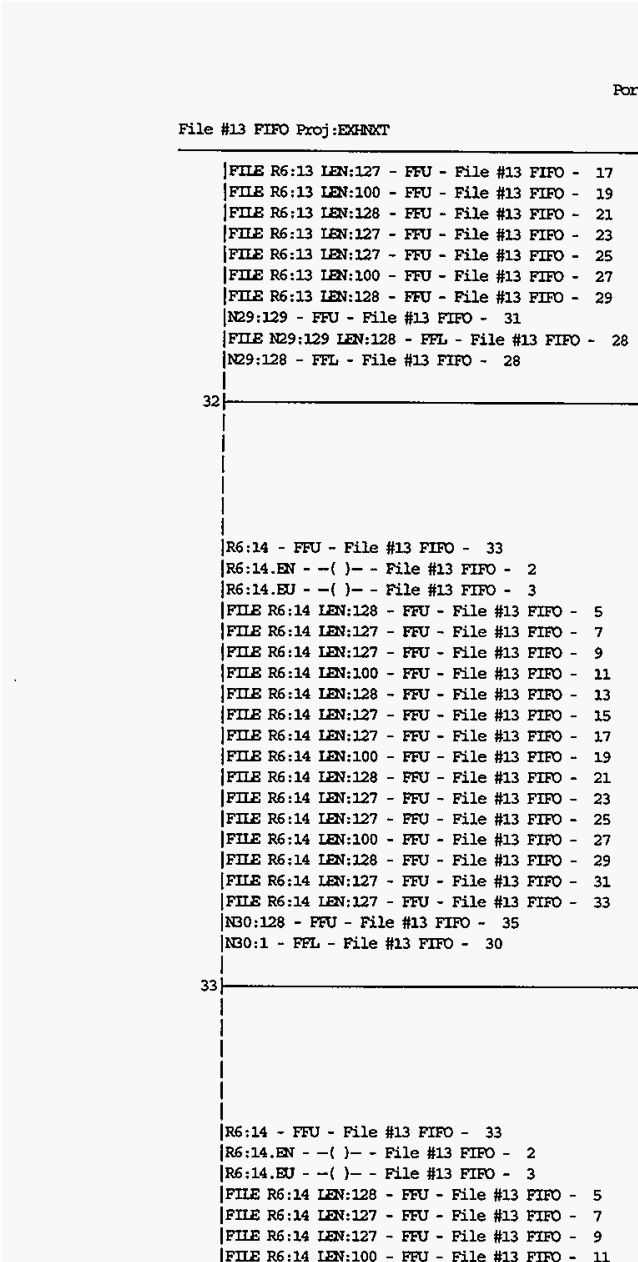

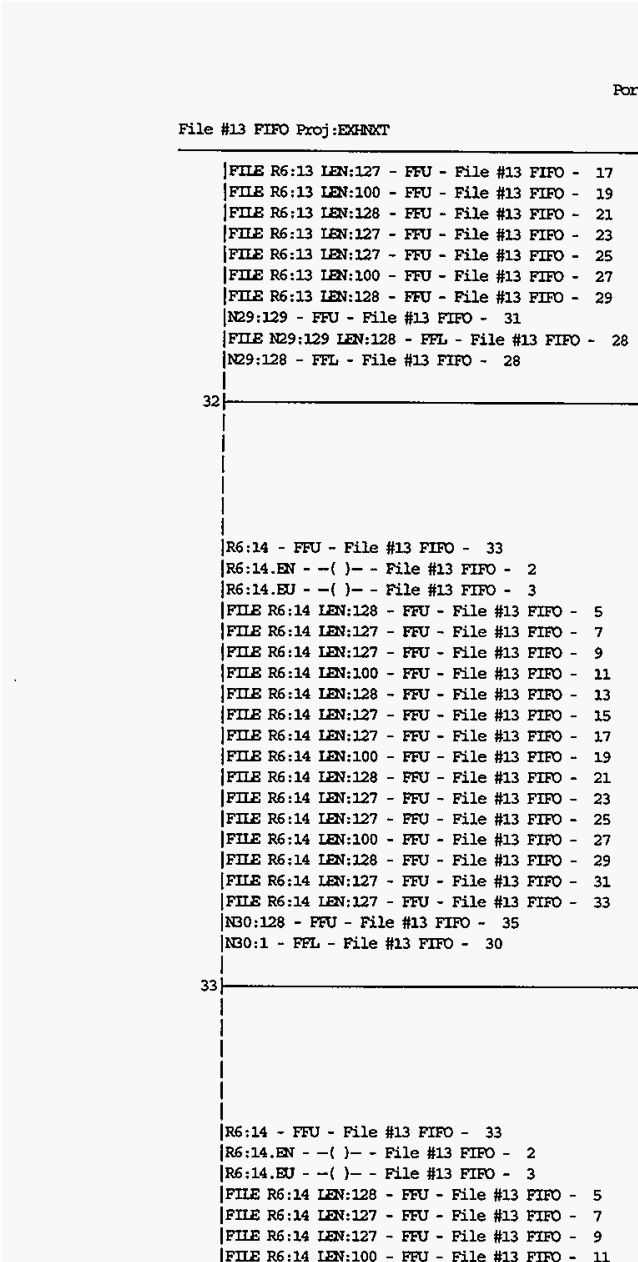

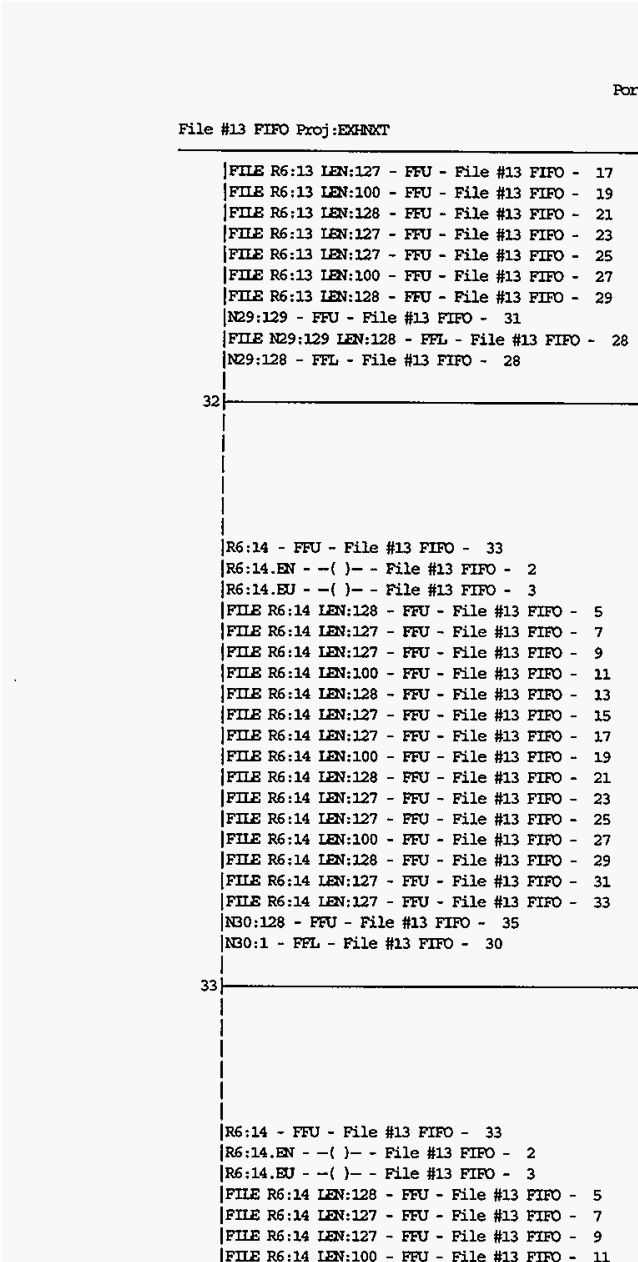

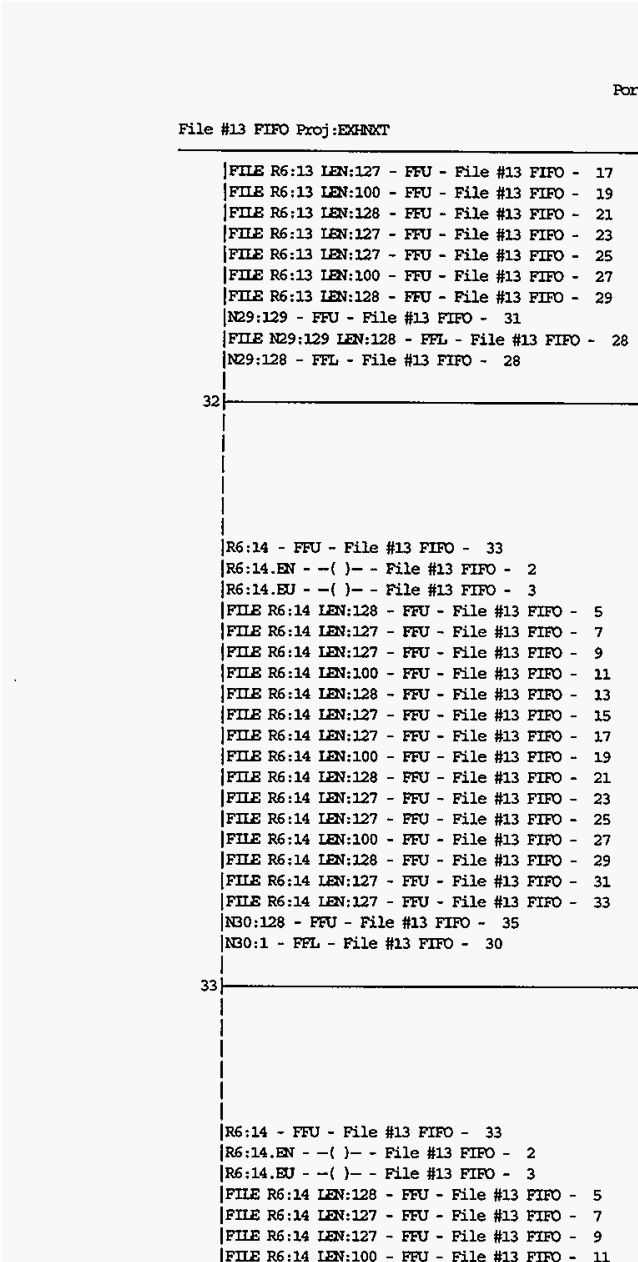

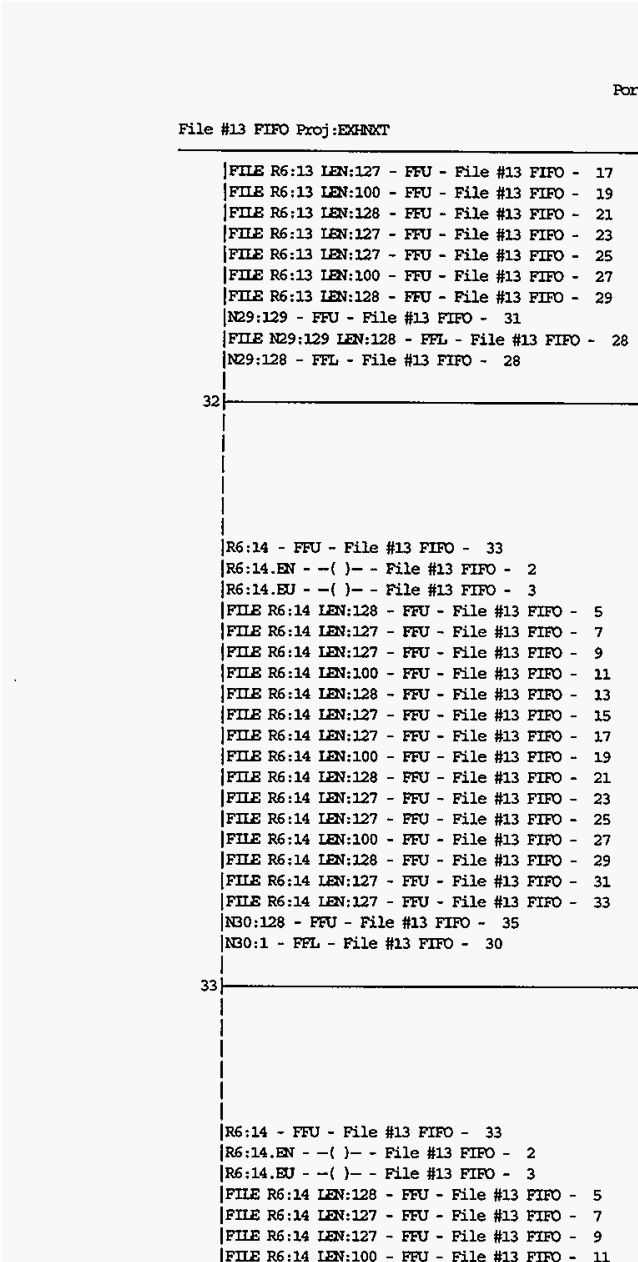

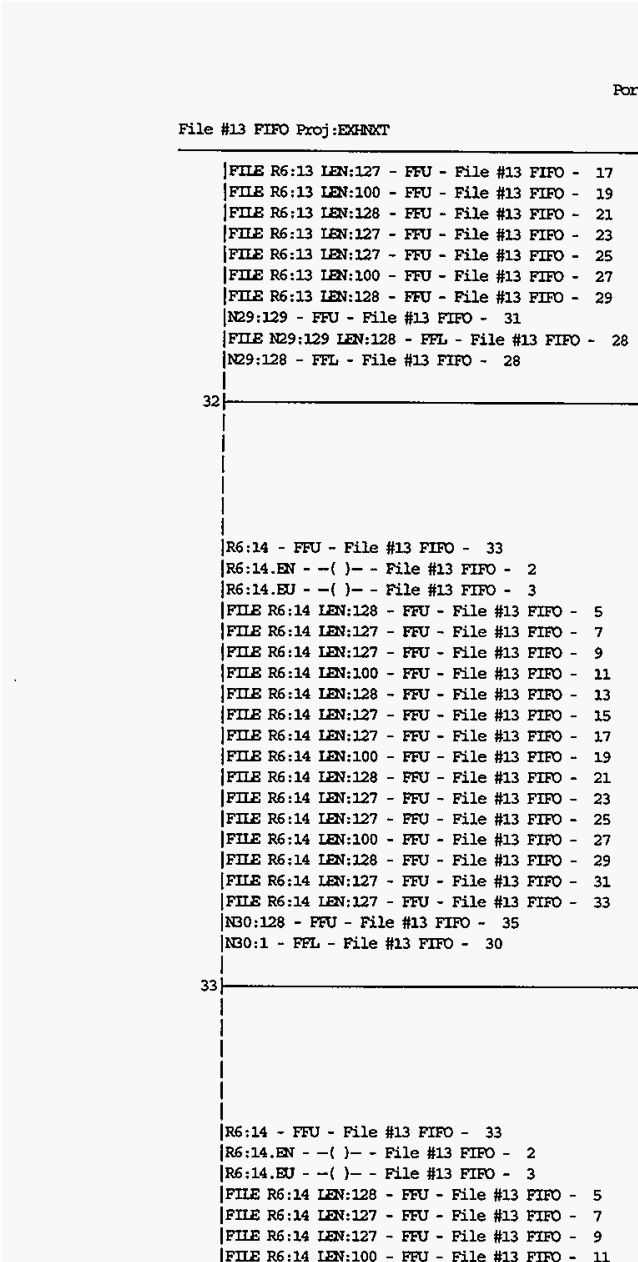

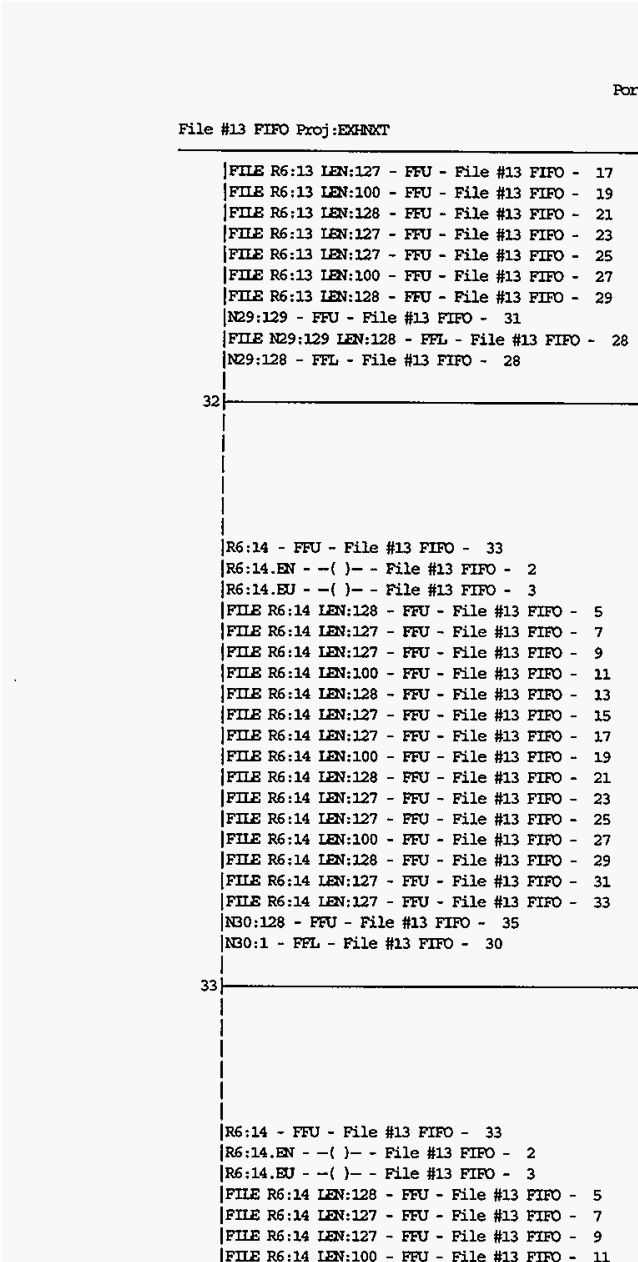

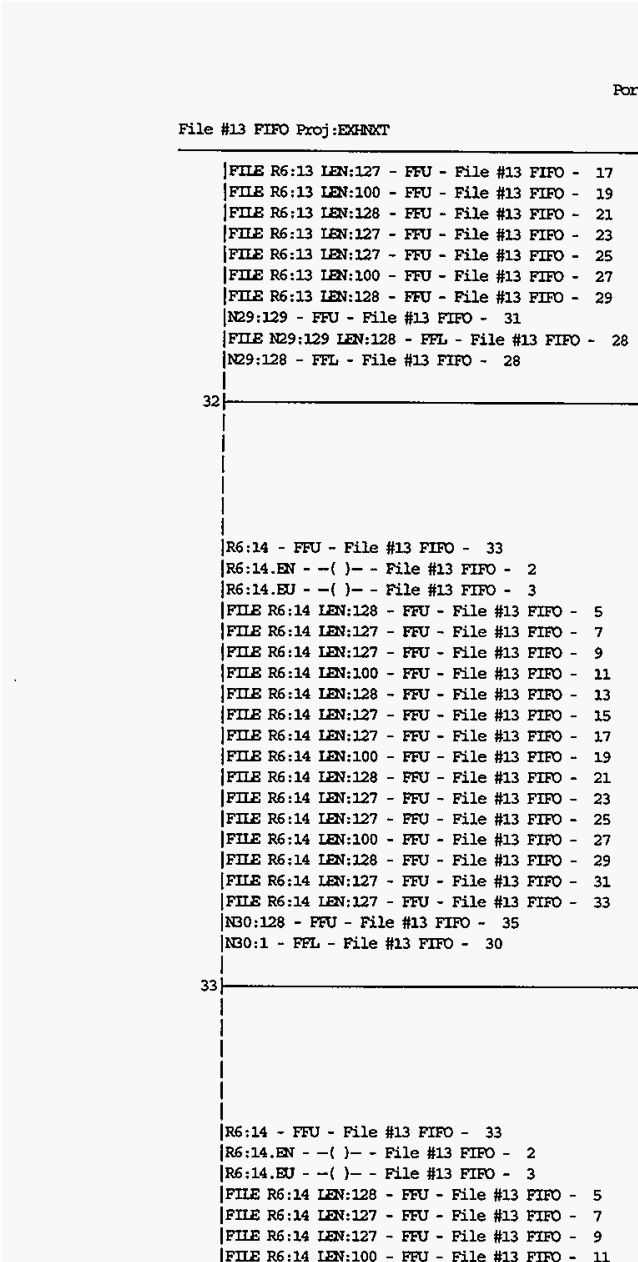

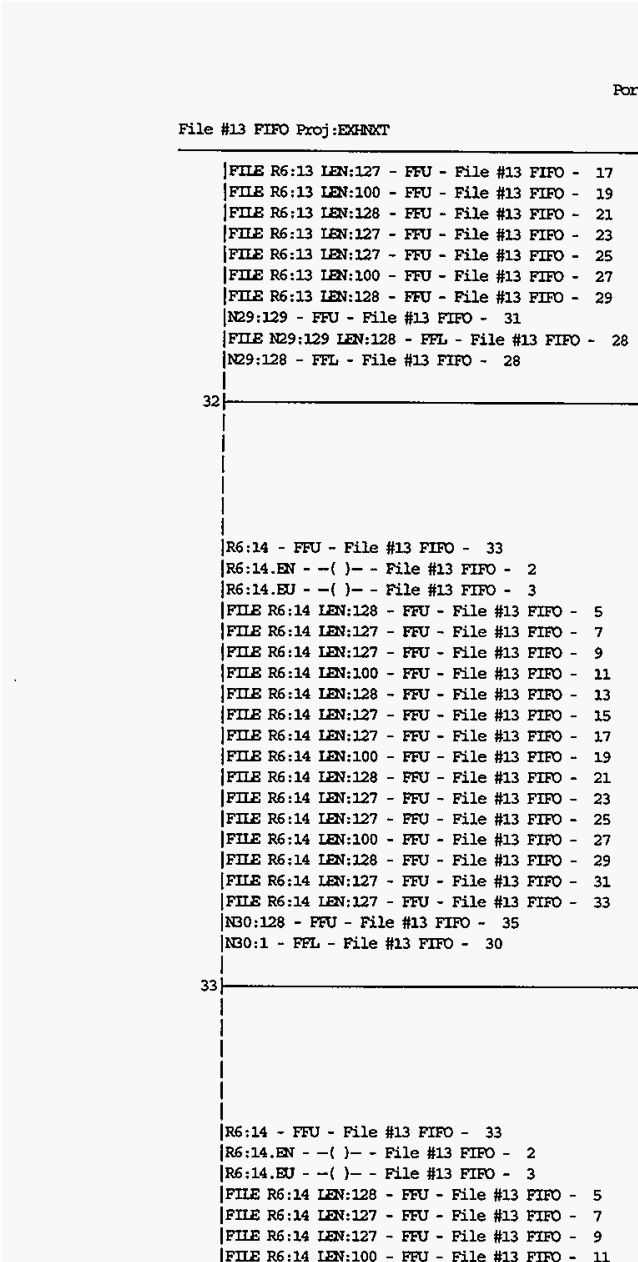

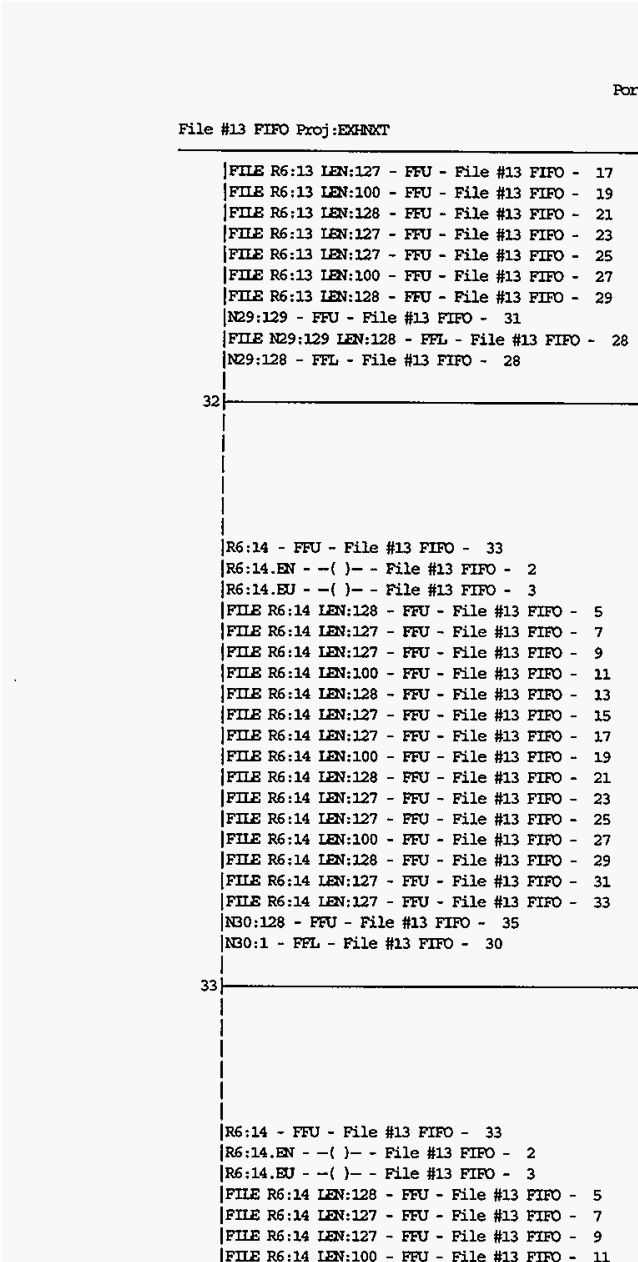

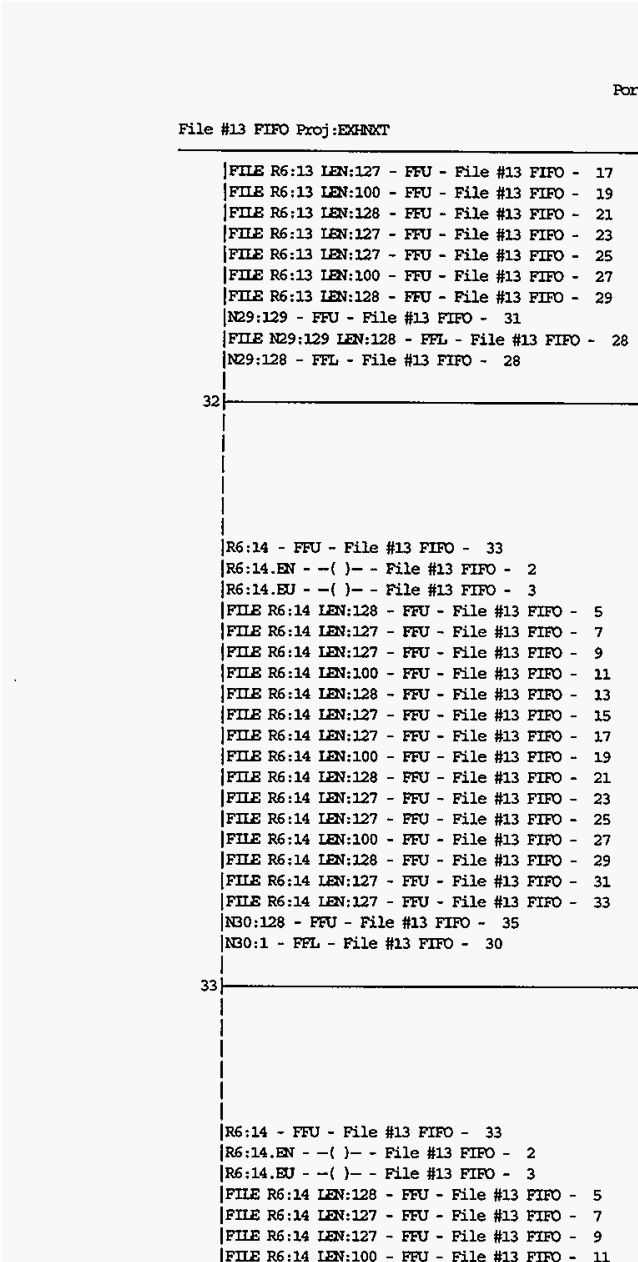

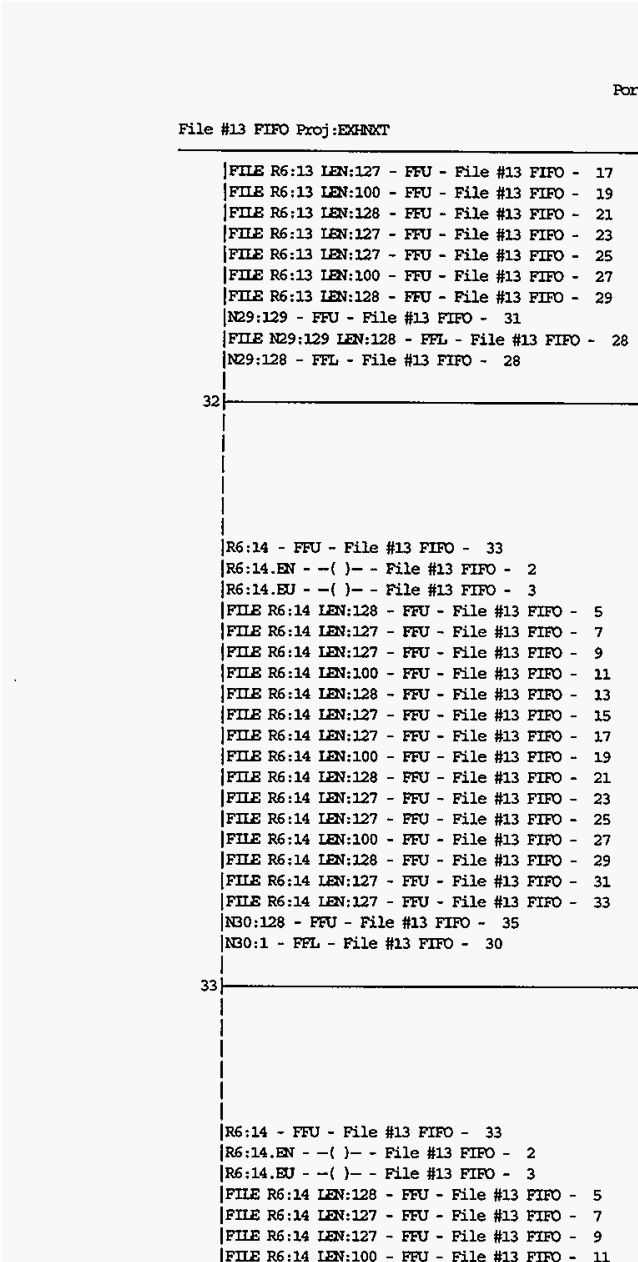

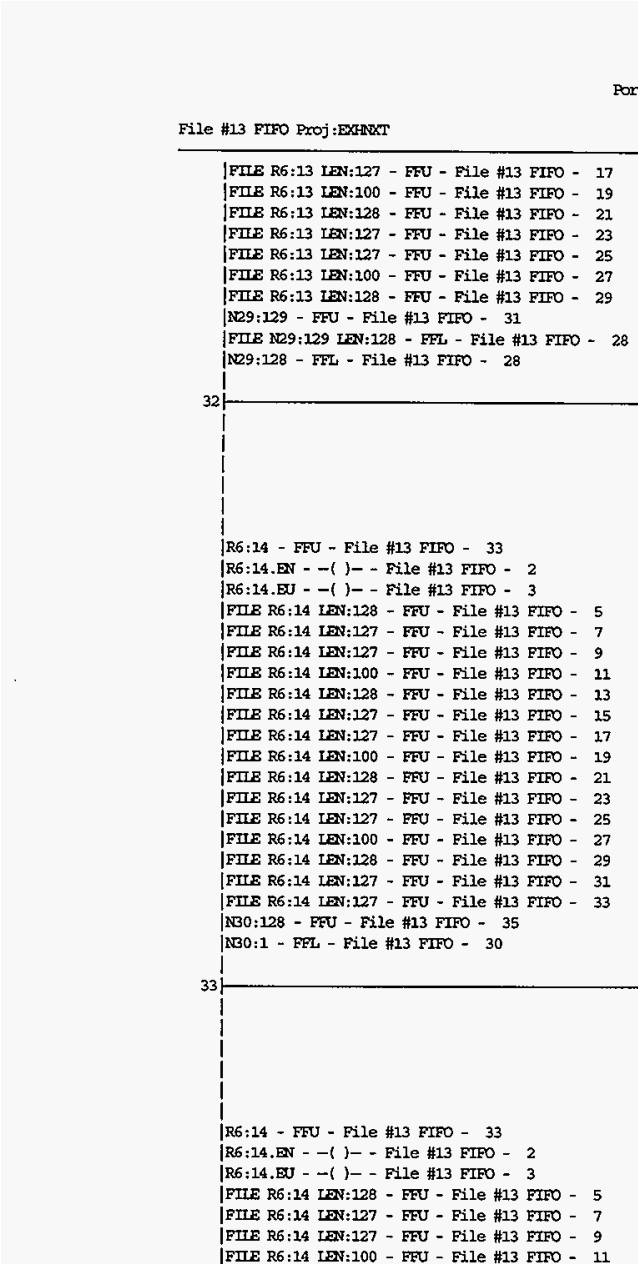
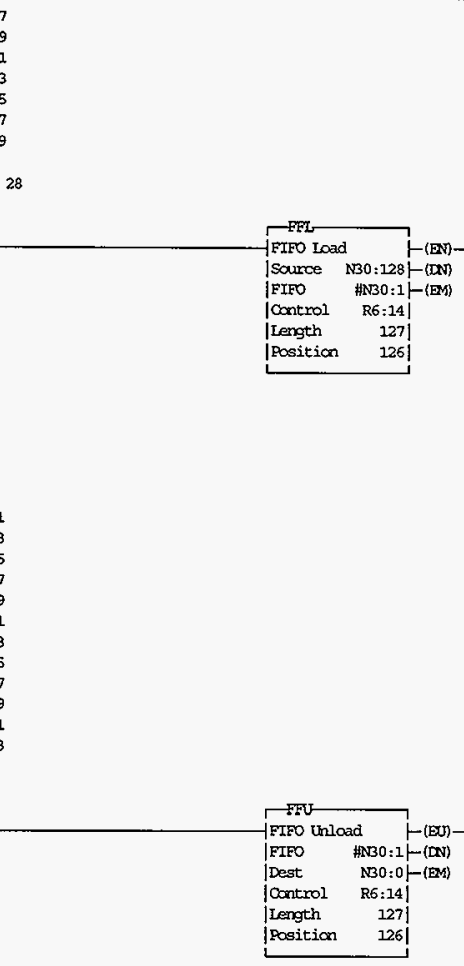

R6:14 - FFL - File \#13 FIFO NB0:128 - FFL - File \#13 FTFO N30:1 - FFL - File $\# 13$ FIFO |N30:1 - FFL - Fil FWU - File \#13 FIEO 33

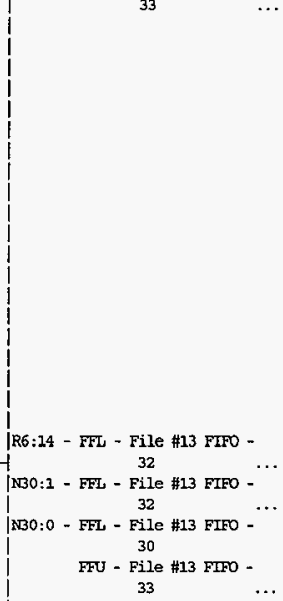

R6:15 - FFL - File \#13 FTFO FUIE S:40 LEN: 100 -

FFL - File \#13 FIFO - 18. . N30:129 - FFL - File \#13 FIFO 34

FNU - File \#13 FIFO -

35 $\ldots$ 
|R6:15 - FFU - File \#13 FIFO - 35

|R6:15.EN - - ( )- - File \#13 FIFO - 2

|R6:15.EU - - ( ) - File \#13 FIFO - 3

|FITE R6:15 LON:128 - FFU - File \#13 FIFO - 5

FFIIE R6:15 IEN:127 - FFU - File \#13 FIFO - 7

FIIE R6:15 IEN:127 - FFU - File \#13 FIFO - 9

FIIE R6:15 LEN:100 - FW - File \#13 FIFO - 11

|FIIE R6:15 LDN:128 - FFU - File \#13 FIFO - 13

|FIIE R6:15 LEN:127 - FFU - File \#13 FIFO - 15

FIIE R6:15 IEN:127 - FFU - File \#13 FIFO - 17

|FIIE R6:15 LEN:100 - FFU - File \#13 FIFO - 19

|FIIE R6:15 LEN:128 - FFU - File \#13 FIFO - 21

|FIIE R6:15 LEN:127 - FFU - File \#13 FLFO - 23

|FIIE R6:15 IEN:127 - FFU - File \#13 FIFO - 25

|FIIE R6:15 IDN:100 - FRU - File \#13 FIFO - 27

|FIIE R6:15 IEN:128 - FEU - File \#13 FIFO - 29

|FIIE R6:15 IEN:127 - FEU - File \#13 FIFO - 31

FIIE R6:15 LEN:127 - FFU - File \#13 FIFO - 33

FIIE R6:15 IEN:100 - FEU - File \#13 FIFO - 35

IS:40 - FFL - File \#13 FIFO - 26

[NB0:129 - FFL - File \#13 FIFO - 32

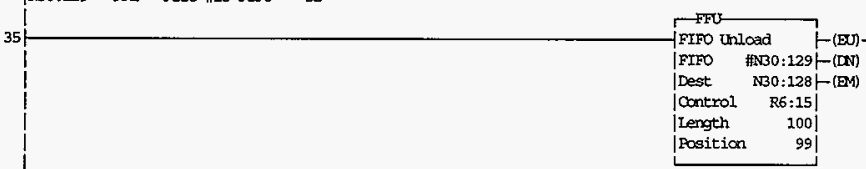

FFr

FIFO

$F$ (BU)

R6:15 - FFU - File \#1.3 FIFO - 35

R6:15.EN - - ( )- - File \#13 FIFO - 2

|R6:15.EJ - - ( )- - File \#13 FIFO - 3

|FIIE R6:15 IDN:328 - FFU - File \#13 FIFO - 5

[FIE R6:15 IEN:127 - FEU - File \#13 FIFO - 7

|FIIE R6:15 IR:127 - FFU - File \#13 FIFO - 9

FIIE R6:15 IEN:100 - FFU - File \#13 FIFO - 11

FFIE R6:15 IEN:128 - FFU - File \#13 FIFO - 13

|FIIE R6:15 IEN:127 - FFU - File \#13 FIFO - 15

|FIIE R6:15 IEN:127 - FFU - File \#13 FIFO - 17

FIIE R6:15 IEN:100 - FFU - File \#13 FIFO - 19

|FIE R6:15 IEN:128 - FFU - File \#13 FIFO - 21

FIIE R6:15 IEN:127 - FNU - File \#13 FIFO - 23

|FILE R6:15 IEN:127 - FFU - File \#13 FIFO - 25

FIIE R6:15 IDV:100 - FFU - File \#13 FIFO - 27

|FILE R6:15 IEN:128 - FFU - File \#13 FIrO - 29

FIIE R6:15 IEN:127 - FEU - File \#13 FIFO - 31

|FIE R6:15 IEN:127 - FFU - File \#13 FIFO - 33

|NB0:129 - FFU - File \#13 FIFO - 35

|FIIE NBO:129 IEN:127 - FFL - File \#13 FIFO - 32

NBO: 128 - FFL - File \#13 FIFO - 32

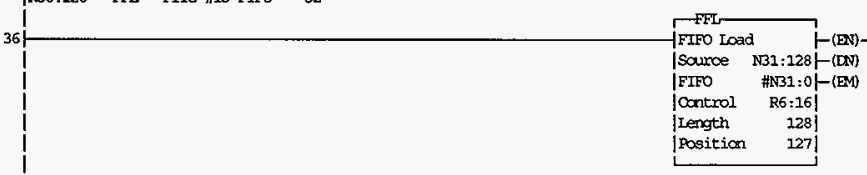

R6:I5 - FF, - File \#13 FIFO |N30:129 - FFL - File \#13 FIFO N30:128 - FFL - File \#13 FIFO 32 Length $\quad 100$ FFU - File \#13 FIFO 35

|R6:16 - FHU - File \#13 FIFO - 37

R6:16.2N - -( )- - Rile \#13 FIFO - 2

R6:16.EJ - - ( ) - File \#13 FIFO - 3

|FIEE R6:16 IEN:128 - FFU - FILe \#13 FIFO - 5

FIIE R6:16 IEN:127 - FFU - File \#13 FTFO - 7 FIIE R6:16 IEN:127 - FFU - File \#13 FIFO - 9 FIIE R6:16 LEN:100 - FNU - File \#13 FIFO - 11 FIIE R6:16 IEN:128 - FFU - File \#13 FIFO - 13 FIIE R6:16 IEN:127 - FFU - File \#13 FIFO - 15 FIIE R6:16 IEN:127 - FEU - File \#13 FIFO - 17 FIIE R6:16 IEN:100 - FFU - File \#13 FIFO - 19 FIIE R6:16 LEN:128 - FFU - File \#13 FIFO - 21 |FIIE R6:16 IEN:127 - FFU - File \#13 FIFO - 23 FILE R6:16 IDN:127 - FFU - File \#13 FIFO - 25 |FIIE R6:16 IEN:100 - FFU - File \#13 FIFO - 27 
|FDE R6:16 LDV:128 - FFU - File \#13 FIFO - 29 |FIIE R6:16 LEN:I27 - FFU - File \#I3 FIFO - 31 |FIE R6:16 IEN:127 - FFU - File \#13 FIFO - 33 |FUE R6:16 LEN:100 - FNU - File \#13 FIFO - 35 |FIIE R6:16 ENN:128 - FFU - File \#13 FTPO - 37 |N31:128 - FFU - File \#13 FIFO - 39

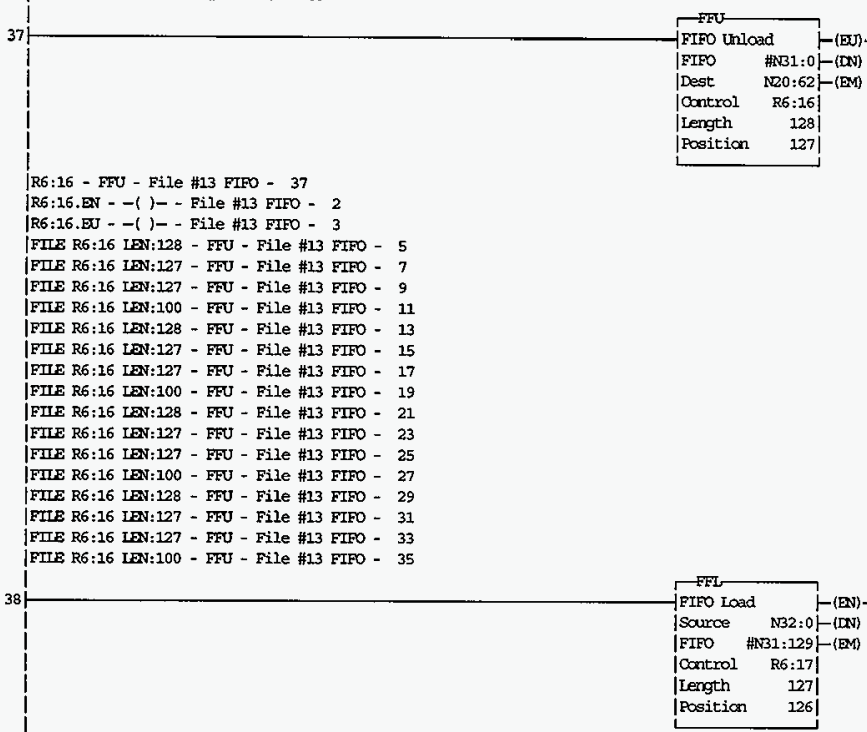

|R6:17 - FFU - File \#13 FIFO - 39

R6:17.EN - - ( ) - File \#13 FIFO - 2

|R6:17.EU - - ( )- - File \#13 FIFO - 3

|FTIE R6:17 IEN:128 - FFU - File \#13 FIFO - 5

FIIE R6:17 IEN:127 - FFU - File \#13 FIFO - 7

|FIIE R6:17 IEN:127 - FFU - File \#13 FIFO - 9

|FIIE R6:17 IEN:100 - FFU - FIIe \#13 FIFO - 11

|FIIE R6:17 IEN:128 - FFU - File \#13 FIFO - 13

|FILE R6:17 LEN:127 - FFU - File \#13 FIFO - 15

|FILE R6:17 IEN:127 - FFU - File \#13 FIFO - 17

|FIIE R6:17 IEN:100 - FFU - File \#13 FIFO - 19

|FIIE R6:17 IEN:128 - FFU - File \#13 FIFO - 21

|FII R6:17 IEN:127 - FFU - File \#13 FIFO - 23

|FIIE R6:17 LAN:127 - FFU - File \#13 FIFO - 25

|FIIE R6:17 IEN:100 - EFU - File \#13 FIEO - 27

|FILE R6:17 LEN:128 - FFU - File \#13 FIFO - 29

|FIL R6:17 LEN:127 - FFU - File \#13 FIFO - 31

|FIIE R5:17 LEN:127 - FFU - File \#13 FIFO - 33

|FIIE R6:17 IEN:100 - EFU - File \#13 FIFO - 35

|FIIE R6:17 IEN:128 - FFU - File \#13 FIFO - 37

|FIE R6:17 IEN:127 - FFU - File \#13 FIFO - 39

|N32:0 - FFU - File \#13 ELFO - 41

IN31:129 - FFL - File \#13 FIFO - 36

9
3 .

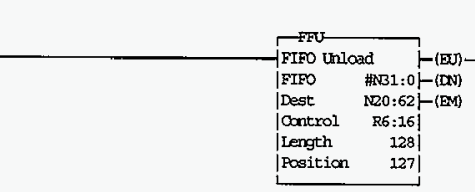

|R6:16 - FFL - File \#13 FIFO -

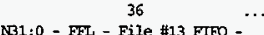 36 FFU - File \#13 FIFO - 37

FII: N20:62 IEN:100 EFL - File \#13 FIFO - 10

R6:17 - FFL - File \#13 FTFO 38 N32:0 - FFL - File \#13 FIFO 38
NB1:129 - FFL - File \#13 FIFO -
38
FFU - File \#13 FIFO -
39 39

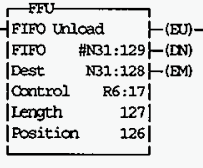

26:17 - FFL - File \#13 FTFO 38 N31:229 - FFL - File \#23 FIro 1 38 N31:128 - FFL - File \#13 FIFO 36 FFU - File \#13 FIFO - 
[R6:17 - FEU - File \#13 FIFO - 39

|R6:17. EN - -( ) - File \#13 FIFO - 2

|R6:17.EU - - ( ) - File \#13 FIFO - 3

|FILE R6:17 LDN:128 - FFU - File \#13 FIFO - 5

|FIIE R6:17 LEN:127 - FFU - File \#13 FIFO - 7

FIIE R5:17 LAN:227 - FFU - File \#13 FIPO - 9

FIIE R6:17 LEN:100 - FFU - PIIe \#13 FIPO - 11

FIIE R6:17 LEN:128 - FFU - File \#13 FIFO - 13

FIIE R6:17 IAN:127 - FFU - File \#13 FIFO - 15

|FIIE R6:17 LAN:127 - FFU - File \#13 FIFO - 17

FIIE R6:17 LEN:100 - FFU - File \#13 FIPO - 19

FHE R6:17 LEN:128 - FFU - File \#13 FIFO - 21

|FIIE R6:17 LEN:127 - FFU - File \#13 FIFO - 23

FTIE R6:17 LEN:127 - FFU - File \#13 FIPO - 25

FTLE R6:17 LAN: 100 - FFU - File \#13 FTPO - 27

|FLLE R6:17 LEN:128 - ENU - File \#13 FIBO - 29

FILE R6:17 LDN:127 - FFU - File \#13 FIFO - 31

FILE R6:17 LDN:127 - FFO - File \#13 FIFO - 33

|FIIE R6:17 LEN: 100 - FFU - File \#13 FIFO - 35

|FILE R6:17 LFN:128 - FFU - File \#13 FIFO - 37

|N31:129 - FFU - File \#13 FIFO - 39

|FILE NB1:129 IDN:128 - FFL - File \#13 FIFO - 36

|N31:128 - FFL - File \#13 FIFO - 36

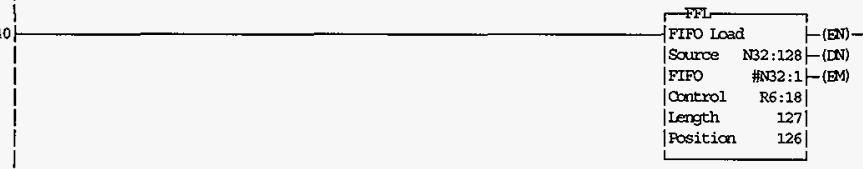

RE:18 - FFL - File \#13 FIFO

R6:18 - FFU - File \#13 FIFO - 41

|R6:18.EN - - ( )- - File \#13 FIFO - 2

R6:18.EJ --()$-$ - File \#13 FIFO - 3

FIIE R6:18 LaN:128 - FFU - File \#13 FIFO - 5

|FIIE R6:18 LEN: 127 - FNU - File \#13 FIFO - 7

FILE R6:18 LEN: 127 - FFU - File \#13 FIBO - 9

FILE R6:18 LEN: 100 - FFU - File \#13 FIFO - 11

FIIE R6:18 LEN:128 - FFU - File \#13 FIFO - 13

|FILE R6:18 IEN:127 - FUU - File \#13 FIFO - 15

|FTE R6:18 IAN:127 - FTU - File \#13 FIFO - 17

|FIIE R6:18 LEN:100 - FFU - File \#13 FIFO - 19

FTHE R6:18 IEN:128 - FFU - File \#13 FIFO - 21

FILE R6:18 IEN:127 - FFU - File \#13 FIFO - 23

FIIE R6:18 IEN:127 - FFU - File \#13 FIFO - 25

|FII8 R6:18 LEN:100 - FFU - File \#13 FIFO - 27

FTIB R6:18 LAN:128 - FFU - File \#13 FIFO - 29

|FTIE R6:18 IDN:127 - FEU - File \#13 FIFO - 31

FIIE R6:18 IEN:127 - FNU - File \#13 FIFO - 33

FIIE R6:18 IEN:100 - FFU - File \#13 FIFO - 35

|FIIE R6:18 IEN:128 - FFU - File \#13 FIFO - 37

FIUE R6:18 IEN:127 - FFU - File \#13 FIFO - 39

|FIIE R6:18 IEN:127 - FFU - File \#13 FIFO - 41

N32:128 - FrU - File \#13 FIFO - 43

IN32:1 - FEL - File H13 FIEO - 38

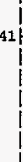

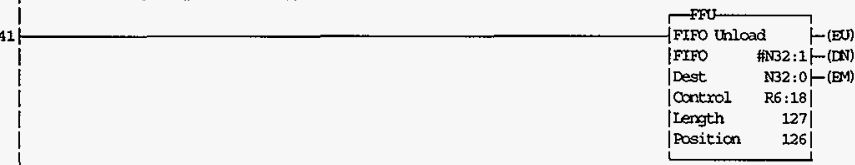

R6:18 - FFU - File \$13 FIFO - 41

R6:18.EN - - ( ) - File \#13 FIFO - 2

R6:18.EJ - - ( ) - File \#13 FIFO - 3

FIE R6:18 LEN:128 - FEU - File \#13 FLFO - 5

FIIE R6:18 IEN:127 - FFU - File \#13 FIFO - 7

|FILE R6:18 IEN:227 - FEU - File \#13 FIFO - 9

FTIE R6:18 IEN:100 - FFU - File \#13 FIFO - 11

FTIE R6:18 IDN:128 - FFU - Pile \#13 FIFO - 13

FITE R6:18 IDN:127 - FFU - File \#13 FIFO - 15

|FIE R6:18 IDN:127 - FFU - File \#13 FIFO - 17

FIFO Unload

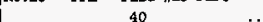

|N32:1 - FFL - File \#13 FIFO -

N32:0 - FFL - File \#23 FIFO -

38

N32:128 - FFL - File \#13 FIFO
40
N32:1 - FFL - File \#13 FIFO -
40
FFU - File \#13 FIFO -
41

FFU - File \#13 FIEO -

41.

HNF-2934

Rev 0

A- 82 
|FIIE R6:18 IEN:100 - FFU - File \#13 FIFO - 19 |EIIB R6:18 IEN:128 - FFU - File \#13 FIFO - 21 FIIE R6:18 IDN:127 - FFU - File \#13 FIFO - 23 |FIIB R6:18 IEN:127 - EFU - Eile \#13 FIFO - 25 FIIE R6:18 IDN:100 - EFU - File \#13 FIFO - 27 FIIE RS:18 IEN:128 - FEU - File \#13 FIFO - 29 FILE R6:18 IEN:127 - FFU - File \#13 FIFO - 31 FULE R6:18 IEN:127 - FFU - File \#13 FIFO - 33 |FITE R6:18 IEN:100 - FFU - File \#13 FIFO - 35 |FIIE R6:18 IDN:128 - FTU - File \#13 FIFO - 37 |EIIE R6:18 IEN:127 - FFU - File \#13 FIFO - 39 |N32:1 - FFU - File \#13 FIFO - 41 |FIIE NB2:1 IEN:127 - FFL - File \#13 FIFO - 38 N32:0 - FFL - File \#13 FIFO - 38

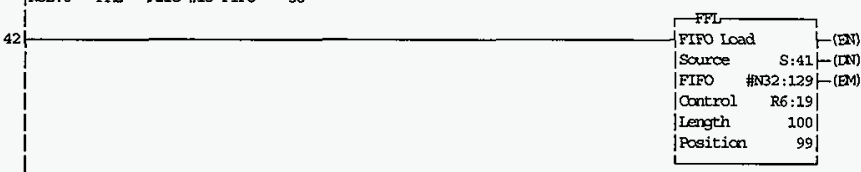

R6:19 - FFU - File \#13 FTFO - 43

R6:19.2N - - ( ) - - File \#13 EIRO - 2

|R6:19.EU - - ( ) - File \#13 FIFO - 3

|FIE R6:19 LEN:128 - FEU - File \#13 FIFO - 5 FIIE R6:19 LEN:127 - FFU - File \#13 FHFO - 7 IFTIE R6-19 IDN:127 - FWU - File \#13 FTFO - 9 FII R6:19 IEN:100 - FFU - File \#13 FIFO - 11 FIIE RE:19 IDN:128 - FFU - File \#13 FIFO - 13 FIIE RG:19 LEN:127 - FRU - File \#13 FIFO - 15 FIIE R6:19 IEN:127 - FFU - File \#13 FIFO - 17 |FIE R6:19 LEN:100 - FFU - File \#13 FIFO - 19 FIIE R6:19 IEN:128 - FFU - File \#13 FIFO - 21 FIIE R6:19 IBN:127 - FFU - File \#13 FIFO - 23 |FTE R6:19 IAN:127 - FEU - File \#13 FIFO - 25 |FIIE R6:19 IRN:100 - FFU - File \#13 FIFO - 27 |FIIE R6:19 IEN:128 - FFU - File \#13 FIFO - 29 |FIE R6:19 IEN:127 - FEU - File \#13 FIFO - 31 |FIIE R6:19 IEN:127 - FEU - File \#13 FIFO - 33 |FIE R6:19 IRN:100 - FEU - File \#13 FTFO - 35 |FIE R6:19 IEN:128 - FEU - File \#13 FTFO - 37 |FIIE R6:19 IEN:127 - FEU - File \#13 FIFO - 39 |FIIE R6:19 IEN:127 - FFU - File \#13 FIFO - 41 |FIIE R6:19 IEN:100 - FEU - File \#13 FIFO - 43 |S:41 - FFL - File \#13 FTFO - 26

FTIE S:41 IEN:100 - FFL - File \#13 FIFO - 34 IN32:129 - FFL - File \#13 FIFO - 40

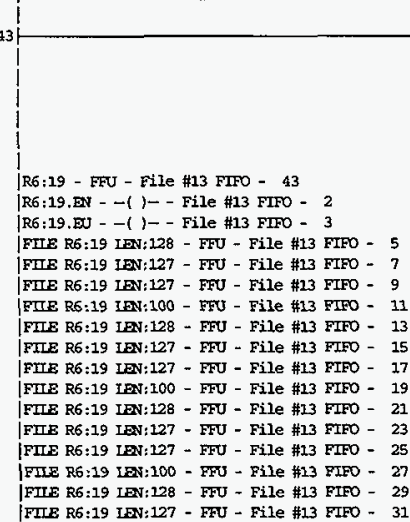

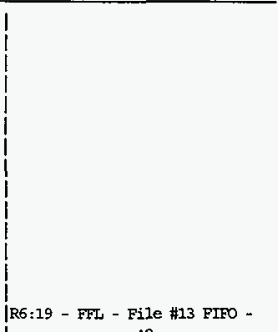

FTI $5: 41$ rot: 42 FFL - File \#13 FIFO - 18. IN32:129 - FFL - File \#13 FIFO 42 FFU - File \#13 FIFO 43

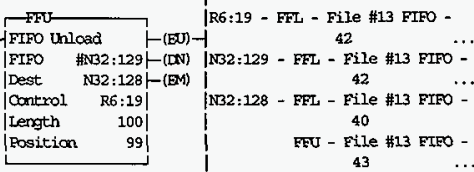

43 
|FHE R6:19 IDN:127 - FFU - File \#13 FIFO - 33 |FIIE R6:19 IEN:100 - FFU - File \#13 FIFO - 35 |FIIE R6:19 LEN:128 - FFU - File \#13 FIFO - 37 |FIIE R6:19 IEN:127 - FFU - File \#13 FIFO - 39 |FIIE R6:19 LEN:127 - FFU - File \#13 FIFO - 41 IN32:129 - FFU - File \#13 FIFO - 43 |FIIE N32:129 IEN; 127 - FFL - File \#13 FIFO - 40 |N32:228 - FEL - File \#13 FIFO - 40

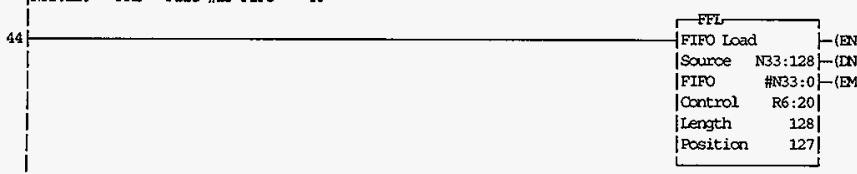

|R6:20 - FFU - File \#13 FIFO - 45

|R6:20.EN - - ( )- File \#13 FIFO - 2

|R6:20.EU - - ( ) - File \#13 EIFO - 3

|FIIE R6:20 INN:128 - FUU - File \#13 FIFO - 5 |FIIE R6:20 IEN:127 - FEU - File \#13 FIFO - 7 |FUE R6:20 IEN:127 - FEU - File \#13 FIFO - 9 |FIE R6:20 IDN:100 - FEU - File \#13 FIFO - 11 |FIIE R6:20 IBN:128 - FEU - File \#13 FIFO - 13 |FITE R6:20 LEN:127 - FFU - FIle \#13 FIFO - 15 |FIIE R6:20 LEN:127 - FFU - File \#13 FIFO - 17 |FIIE R6:20 LEN:100 - FFU - File \#13 FIFO - 19 FUE R6:20 LEN:128 - FFU - File \#13 FIFO - 21 |FIE R6:20 IFN:127 - FEU - File \#13 FIFO - 23 |FILE R6:20 IEN:127 - FEU - File \#13 FTFO - 25 |FIIE R6:20 IDN:100 - FEU - File \#13 FIFO - 27 |FILE R6:20 IEN:128 - FEU - File H13 FIFO - 29 |FILE R6:20 IDF:127 - FVU - File \#13 FTFO - 31 |FIE R6:20 IBN:127 - FEU - File \#13 FIFO - 33 |FIIE R6:20 IEN:100 - FFU - File \#13 FIFO - 35 |FILE R6:20 IEN:128 - FEU - File \#13 FIFO - 37 |FITE R6:20 IEN:127 - FEU - File \#13 FIFO - 39 |FIIE R6:20 IDN:127 - FEU - File \#13 FTFO - 41 |FIIE R6:20 I0N:100 - FEU - File \#13 FIFO - 43 |FIIE R6:20 IFN:128 - FFU - File \#13 FIFO - 45 |R33:128 - FFU - File \#13 FTFO - 47<smiles>C[As]</smiles>

(

i

1

IR6:20 - FFU - File \#13 FIFO - 45

[R6:20.EN - - ( ) - File \#13 FIFO - 2

|R6:20.EU - - ( ) - File \#13 FIFO - 3

|FIIE R6:20 IDN:128 - FEU - File \#13 FIFO - 5

|FIIE R6:20 IEN:127 - FEU - File \#13 FIFO - 7

|FTIE R6:20 IEN:127 - FUU - File \#13 FIFO - 9

|FITE R6:20 IEN:100 - FEU - File \#13 FIFO - 11

|FIE R6:20 IEN:128 - FEU - File \#13 FIFO - 13

|FIIE R6:20 IEN:127 - FUU - File \#13 FIFO - 15

|FIE R6:20 IEN:127 - FFU - File \#13 FIFO - 17

FIIE R6:20 IEN:100 - FFU - File \#13 FIFD - 19

FIIE R6:20 LEN:128 - FFU - File \#13 FIFO - 21

FUL R6:20 LEN:127 - FFU - File \#13 FIFO - 23

FTIE R6:20 LEN:127 - FEU - File \#13 FIFO - 25

FFIE R6:20 IEN:100 - FFU - File \#13 FIFO - 27

[FIUE R6:20 IEN:228 - FFU - File \#13 FIFO - 29

FIIE R6:20 IDN:127 - FEU - File \#13 FIFO - 31

|FIE R6:20 LEN:127 - FFU - File \#13 FIFO - 33

FIIE R6:20 IDN:100 - FEU - File \#13 FIFO - 35

|FIIE R6:20 IEN:128 - FFU - File \#13 FIFO - 37

FIUE R6:20 IEN:127 - FFU - File H13 FIFO - 39

FIIE R6:20 IEN:127 - FFU - File \#13 FTFO - 41

|FIIE R6:20 IDN:100 - FFU - File \#13 FIFO - 43
1

|R6:20 - FFL - File \#13 FIPO -

44

|N33:128 - FFL - File \#13 FIFO -

NB3:0 - FFL - File \$13 FIFO -

1 in:0

FFU - Eile \#13 FIFO -

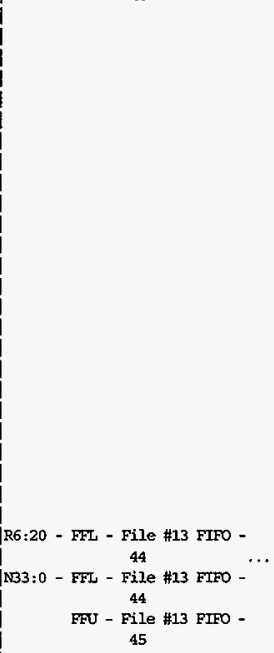

|FIIE N20:63 IEN:100-

FFL - File \#13 FIFO - 10

HNF-2934

Rev 0 


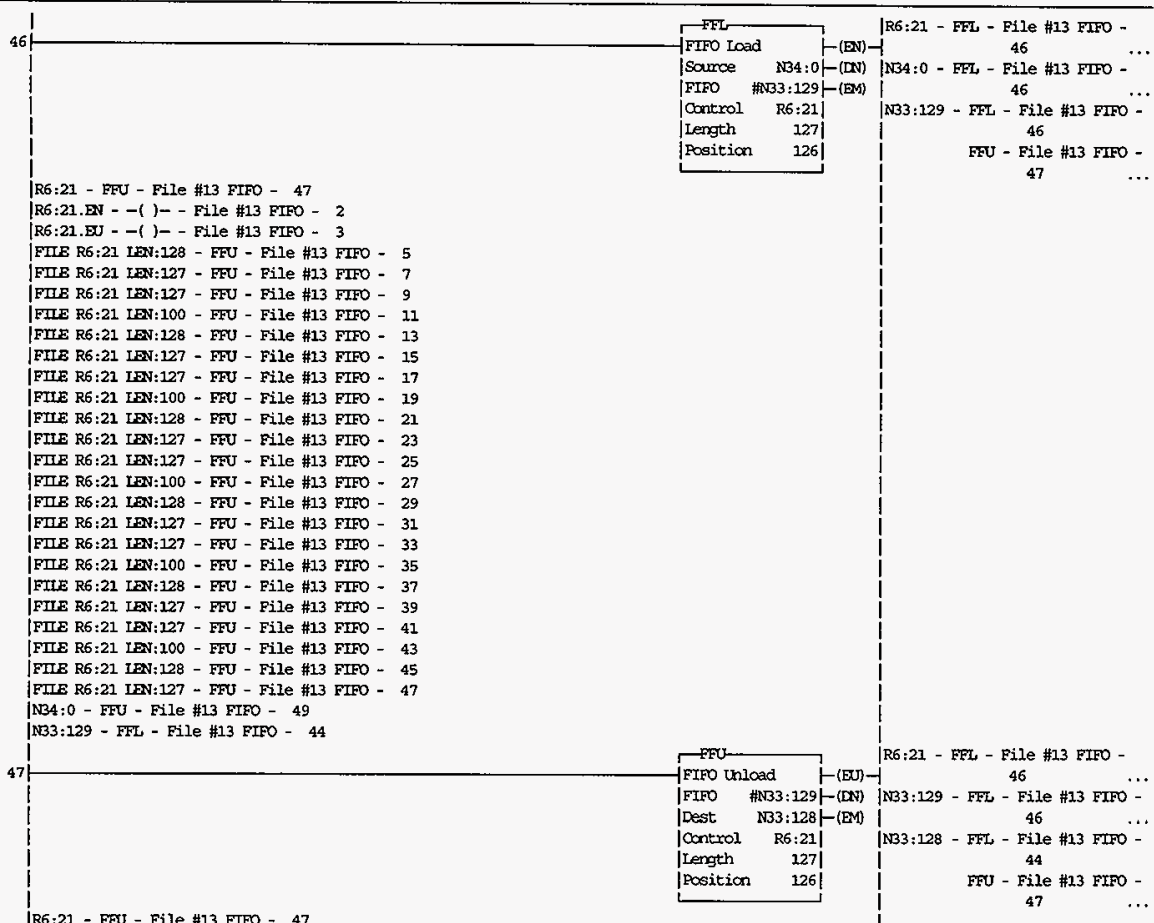

|R6:21.EN - -( ) - File \#13 FIFO - 2

|R6:21.EN - - ( ) - File \#13 FIFO - 3

|FILE R6:21 LEN:128 - FFU - File \#13 FIFO - 5

FIIIE R6:21 IEN:127 - FFU - File \#13 FIFO - 7 FIIE R6:21 IDN:127 - FFU - File \#13 FIFO - 9 FIIE R6:21 IEN:100 - FFU - File \#13 FTFO - 11 FTIE R6:21 IEN:128 - FFU - File \#13 FIFO - 13 |FIIE R5:21 IEN:127 - FFU - File \#13 FIFO - 15 FIIE R6:21 IRN:127 - FFU - File \#13 FIFO - 17 |FIIE R6:21 LEN:100 - FFU - File \#13 FIFO - 19 FIIE R6:21 LEN:128 - FFU - File \#13 FIFO - 21 |FILE R6:21 IDN:127 - FFU - File \#13 FIFO - 23 FחE R6:21 IAN:127 - EFU - File \#13 FIFO - 25 |FIIE R6:21 IEN:100 - FFU - File \#13 FIFO - 27 |FIIE R6:21 IAN:128 - FRU - File \#13 FIFO - 29 |FIIE R6:21 IEN:127 - FFU - File \#13 FIFO - 31 FIIE R6:21 LEN:127 - FFU - EIIE \#13 FIFO - 33 |FIEE R6:21 LEN:100 - EFU - File \#13 FIFO - 35 FIIE R6:21 LEN:128 - FFU - Pile \#13 FIFO - 37 FIIE R6:21 IAN: 127 - FFU - File \#13 FIFO - 39 |FTE R6:21 IEN:127 - ENU - File \#13 FIFO - 41 |FIE R6:21 IEN:100 - FFU - File \#13 FIFO - 43 |FIIE R6:21 IEN:128 - FFU - File \#13 FIFO - 45 |N33:129 - FTU - File \#13 EIFO - 47

|FIIE NB3:129 LEN:128 - FFL - File \#13 FIFO - 44 N33:128 - FFL - File \#13 FIPO - 44 


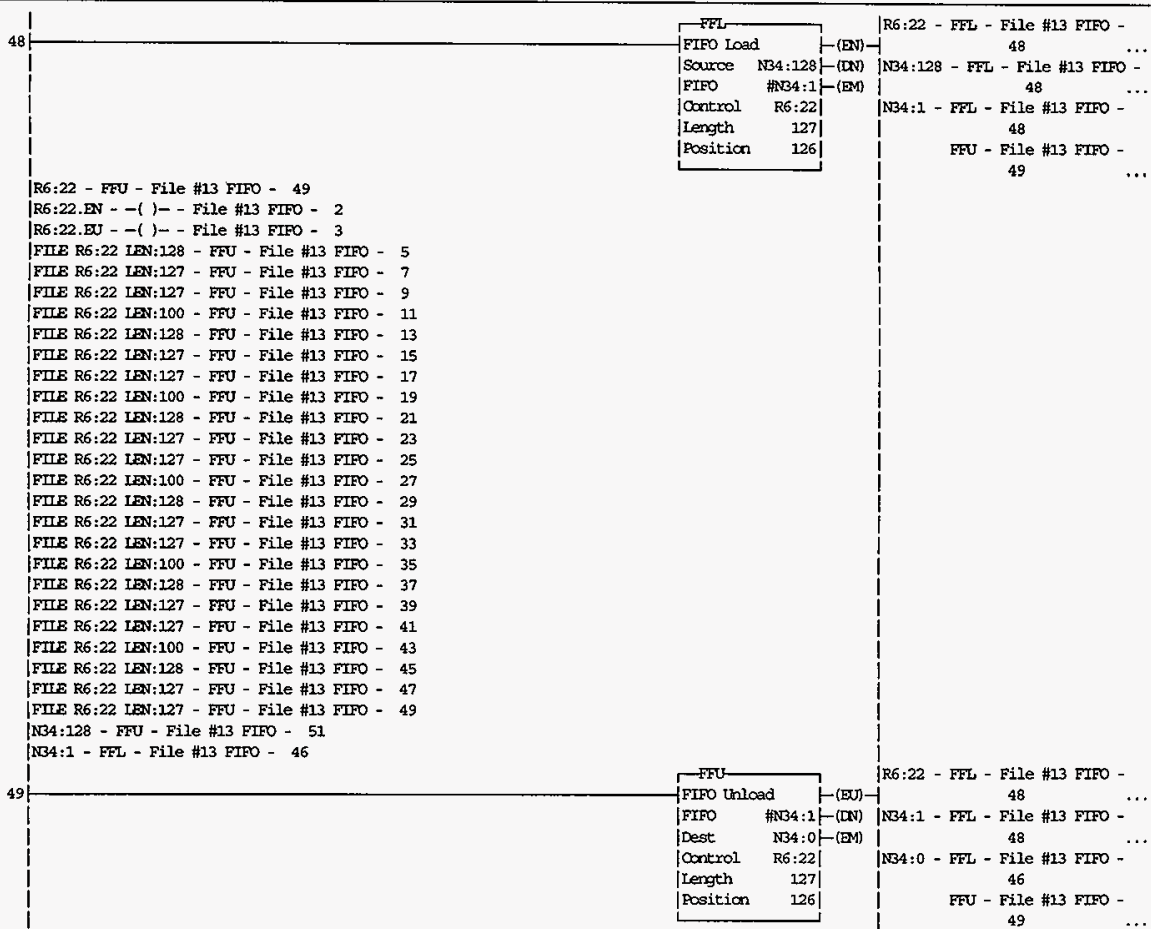

R6:22 - FFU - File \#13 FIFO - 49

|R6:22.EN - - ( )- - File \#13 FIFO - 2

|R6:22.EJ - -( ) - File \#13 FIFO - 3

|FILE R6:22 IDN:128 - FFU - File \#13 FTPO - 5

|FIIE R6:22 IEN:127 - FFU - File \#13 FIFO - 7

|FIIE R6:22 IEN:127 - FFU - File \#13 FIFO - 9

|FIIE R6:22 IFN:100 - FFU - File \#13 FTFO - 11

FTIE R6:22 IDN:128 - FFU - File \#13 FIFO - 13

FNIE R6:22 IEN:127 - FEU - File \#13 FIFO - 15

|FIIE R6:22 IDN:127 - FFU - File \#13 FIFO - 17

FIIE R6:22 IDN:100 - FFU - File \#13 FIFO - 19

FILE R6:22 IRN:128 - FFU - File \#13 FIFO - 21

FIIE R6:22 LEN:127 - FFU - File \#13 FIFO - 23

|FILE R6:22 LEN:127 - FFU - File \#13 FIFO - 25

|FIIE R6:22 IEN:100 - FFU - File \#13 FIFO - 27

|FTIE R6:22 IEN:128 - FEU - File \#13 FIFO - 29

|FUE R6:22 IEN:127 - FU - File \#13 FIFO - 31

|FIE R6:22 IEN:127 - FFU - File \#13 FTFO - 33

FIIE R6:22 IBN:100 - FFU - File \#13 FIFO - 35

|FIIE R6:22 IEN:128 - FFU - File \#13 FIFO - 37

FIIE R6:22 IEN:127 - FFU - File \#13 FIFO - 39

FIIE R6:22 IEN:127 - FFU - File \#13 FIFO - 41

FUIE R6:22 IEN:100 - FFU - File \#13 FIFO - 43

FTIE R6:22 IFN:128 - FFU - File \#13 FIFO - 45

FIIE R6:22 IEN:127 - FFU - File \#13 FIFO - 47

|N34:1 - FFU - File \#13 FIPO - 49

|FIE N34:1 IDN:127 - FFL - File \#13 FIFO - 46

HNF-2934 


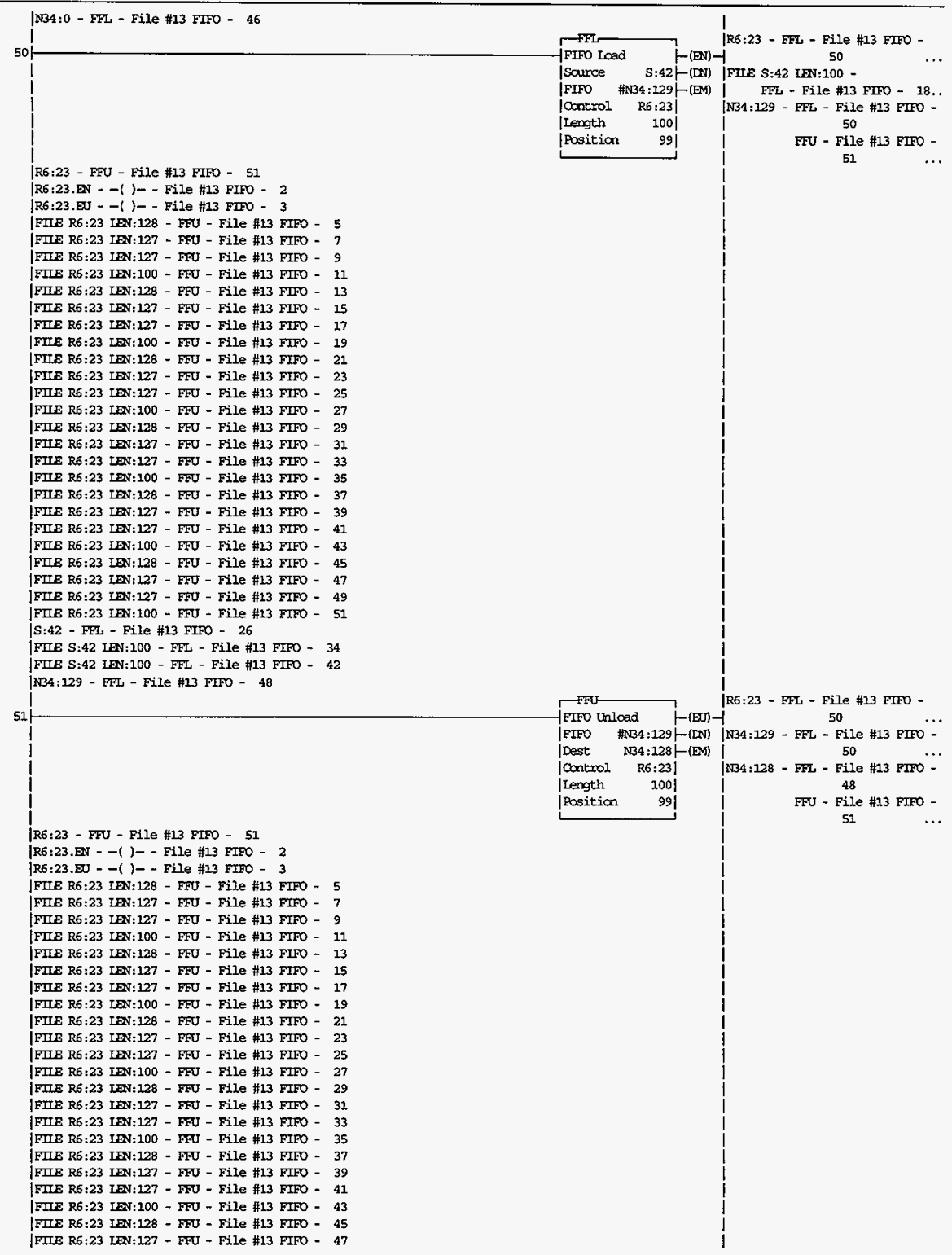

HNF-2934

Rev 0 
|FIIE R6:23 LDN:127 - FFU - File \#13 FIPO - 49

|N34:129 - FFU - File \#13 FIFO - 51

FIIE N34:129 JEN:127 - FFL - Fíle \#13 PIFO - 48

|N34:128 - FFL - File \#13 FIFO - 48

1

52

$\rightarrow$ ton-

531

Return

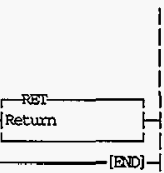




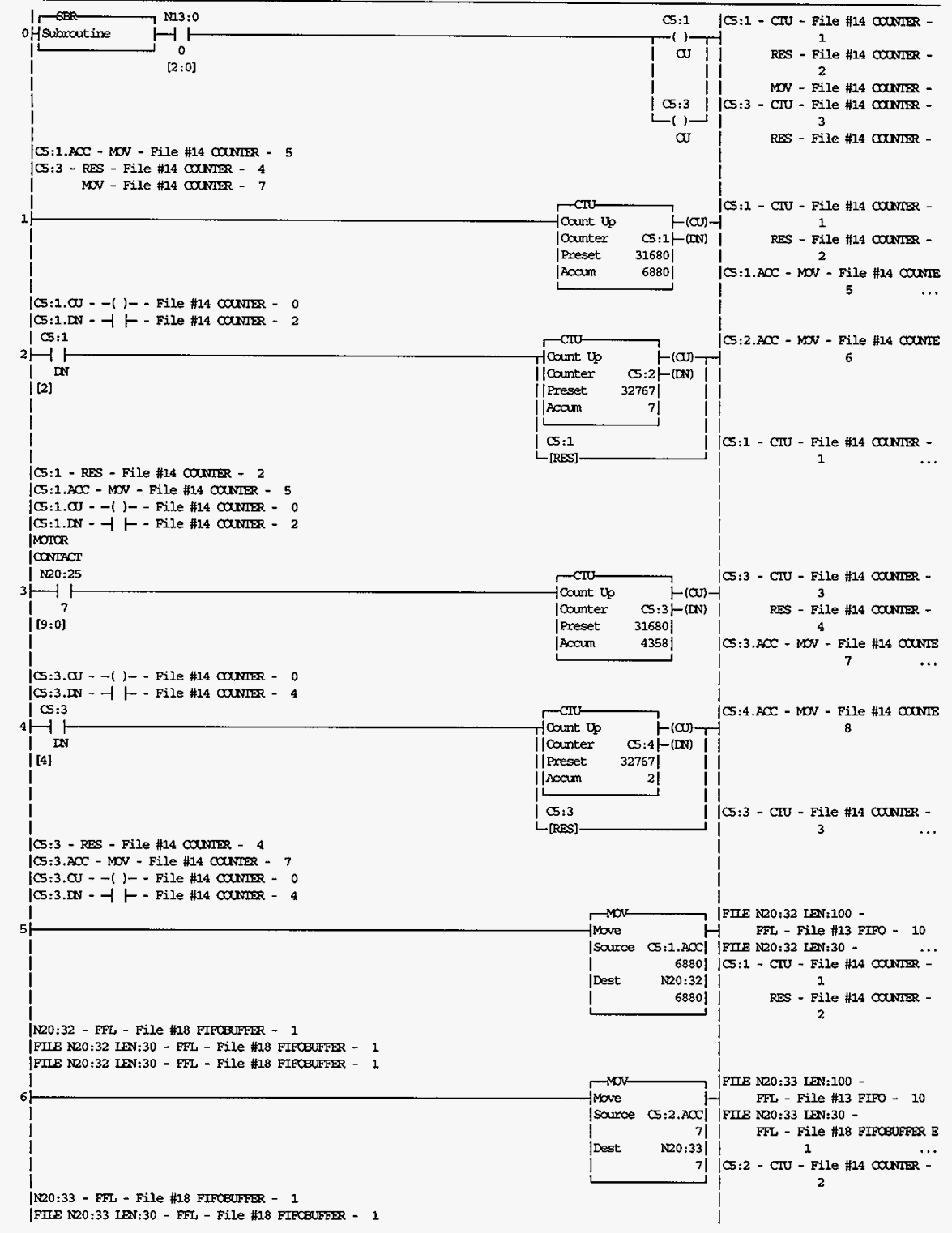




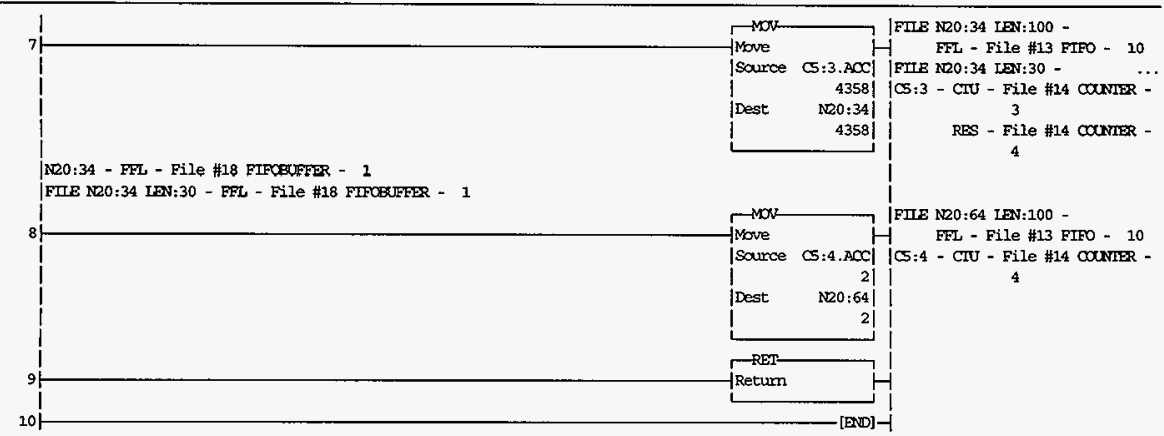

HNF-2934

Rev 0

A-90 


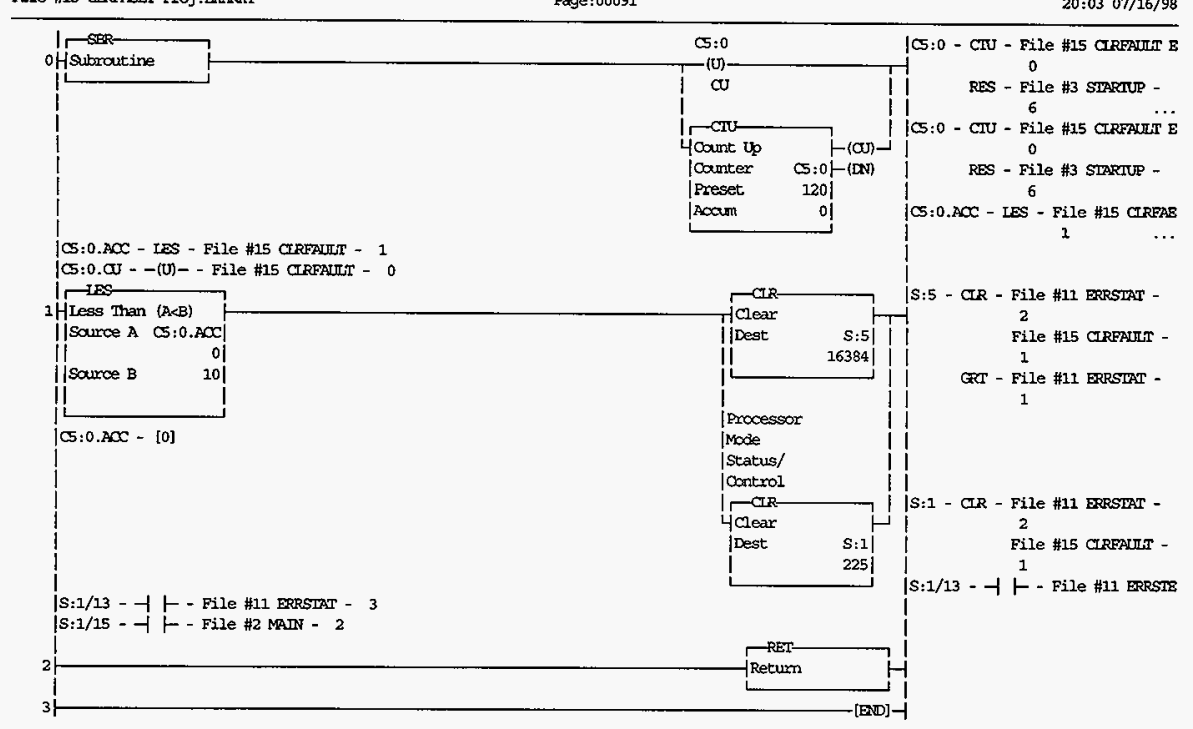




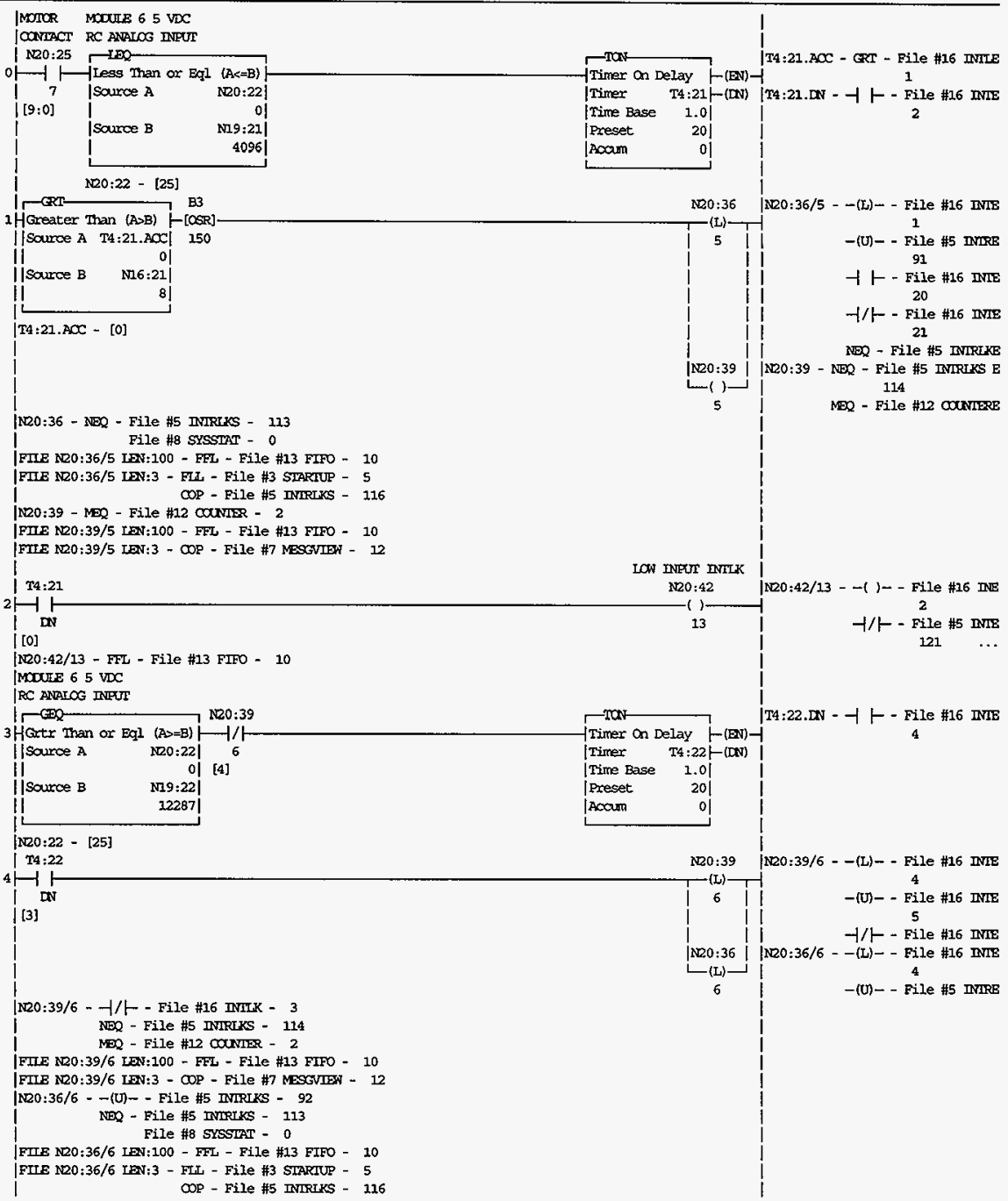


|MOCUIE 65 vDC IRC PNALOS INFT $1-i n-$

I| source $A$ NRO:22| 151

II

||Samce B

이

N1.8:221

9500

$20: 22-[25]$

1N20:39 - MOQ - File \#12 COMIrR - 2

|FILE N20:39/6 LEN:100 - FFL - File \#13 ELFO - 10

|FIE N20:39/6 IEN:3 - OP - File \#7 MESGVIEN - 12

|MOTOR

loarmact

| less Than of Eq1

|T4:23.ACC - [6]

1

1

N20:36 - NEQ - File \#5 INIRIKS - 113

File \#8 SYSSMAr - 0

|FIIE N20:36/7 LEN:100 - ERL - File \#13 FIFO - 10

|FIT A20:36/7 LEN:3 - FUL - File \#3 STARIUP - 5

COP - File \#S TMIRTKS - 116

|N20:39 - MEQ - File \#12 COUNIER - 2

|FIE N20:39/7 LEN:100 - FFL - File \#13 FIFO - 10

[FIIE N20:39/7 LEN:3 - CO - File \#7 MESGIIEN - 12 $\mathrm{T} 4: 23$

8 - 1

I DN

[6]

N20:42/14 - FFL - File \#13 FIFO - 10

$1-c=0$

9HGtx Than or Eql (A>m)

||Somce A N20:23| 8

11

| Scarroe $B$

이 [10]

II 22287

1N20:23 - [27]

I4:24

10-ㅏㄱ 1

I $\mathbf{L N}$

| [9]

1

IN20:39/8 - H/F - File \#16 INITK - 9

NDQ - File \#5 INTRIKS - 114

MER - File \#12 CONIER - 2

|FIIE N20:39/8 LEN:100 - FFL - File \$13 FIFO - 10

|FIIE NQ0:39/8 LEN:3 - COP - File \#7 MESGVIEN - 12

INRO:36/8 - -(U) - - File \#5 INMTKS - 94

NSP - File 45 DNIRIXS - 113

File \#8 SZSSTIT - 0

|FIIE ND0:36/8 IEN:100 - FFL - FIle \#13 FIFO - 10

I

N20:39 [N20:39/6 - -(L)- - File \#16 IIIE 4

-(U) - - File \#16 INIE 5

-1 - File \#16 INIE 3

NED - File \#5 INIRKE 114

T4:23.ACC - GRT - File \#16 TNILE Timer on Delay - (EN)7

Timer T4:23ן(DN) |T4:23. WN - - 1 - Eile \#16 INIE Time Rase 1.0| Preset Acam $\quad 0 \mid$

N20:36/7 - - (L) - File \#16 INIE

N20:36 (L) -

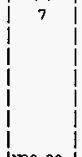

|N20:39 | - ( )
7

-(U) - - File \#5 INIFE 93

$\dashv \vdash-$ File \#16 INIE 20

- $/$ - - File \#16 TME 21

NDQ - File \#5 INIRIX

ND0:39 - NDO - File \#5 INIRLKS E 114

MEQ - File \#12 COINIERE 


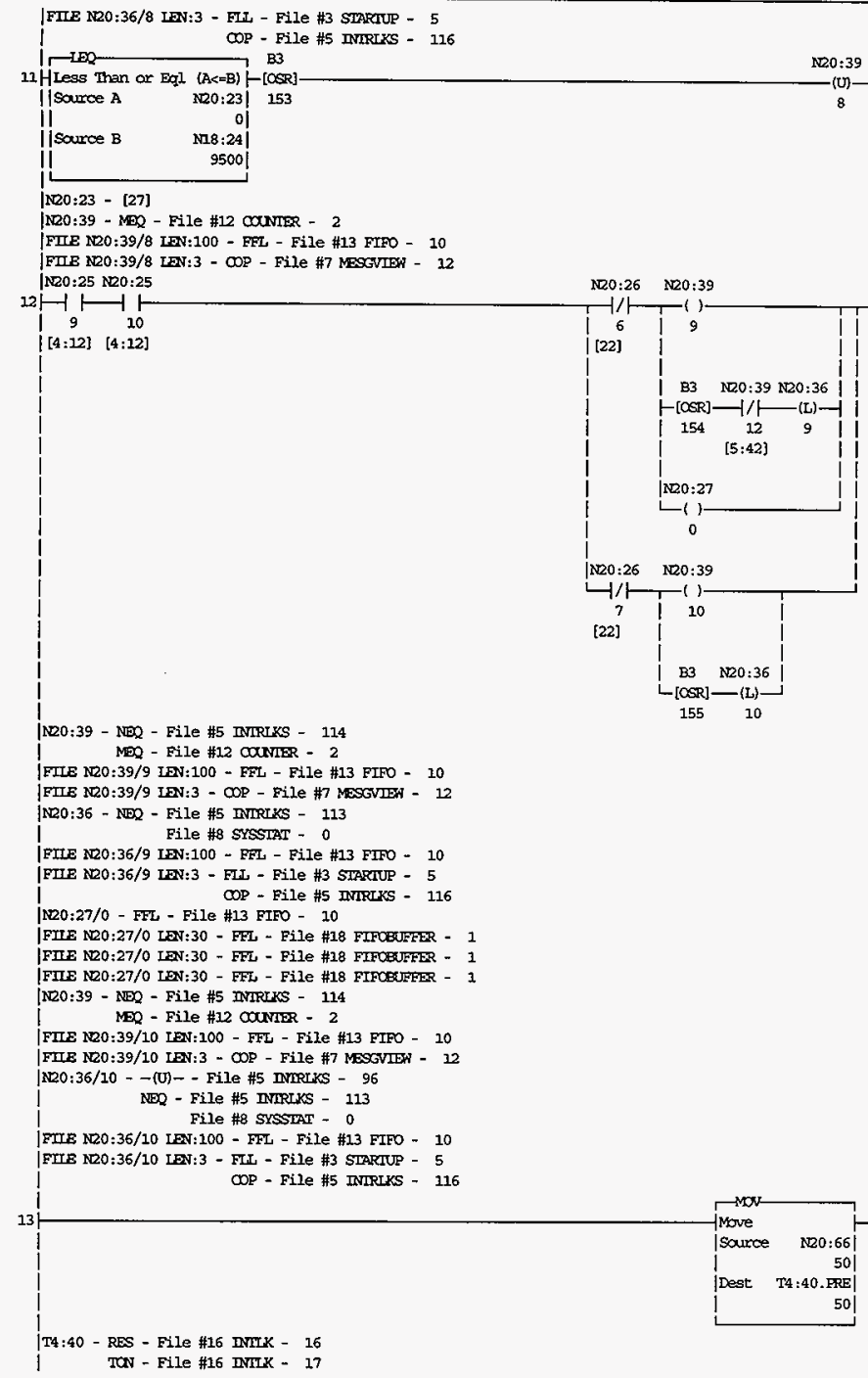




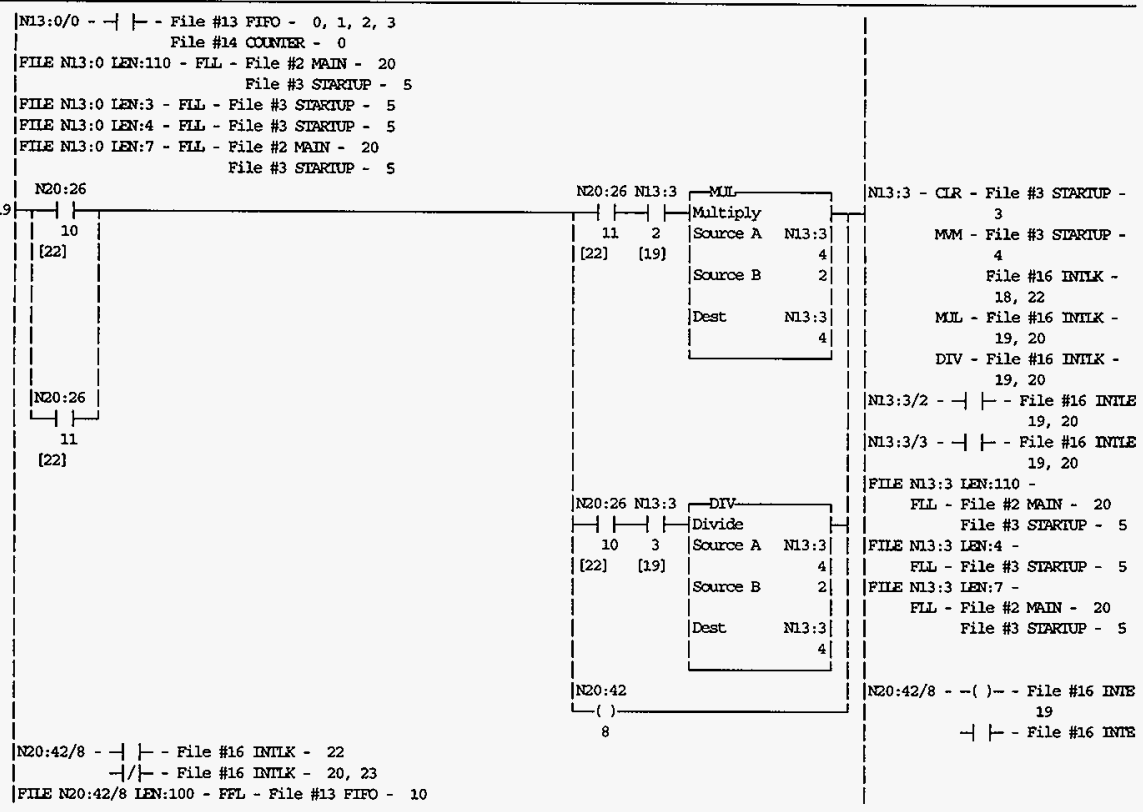




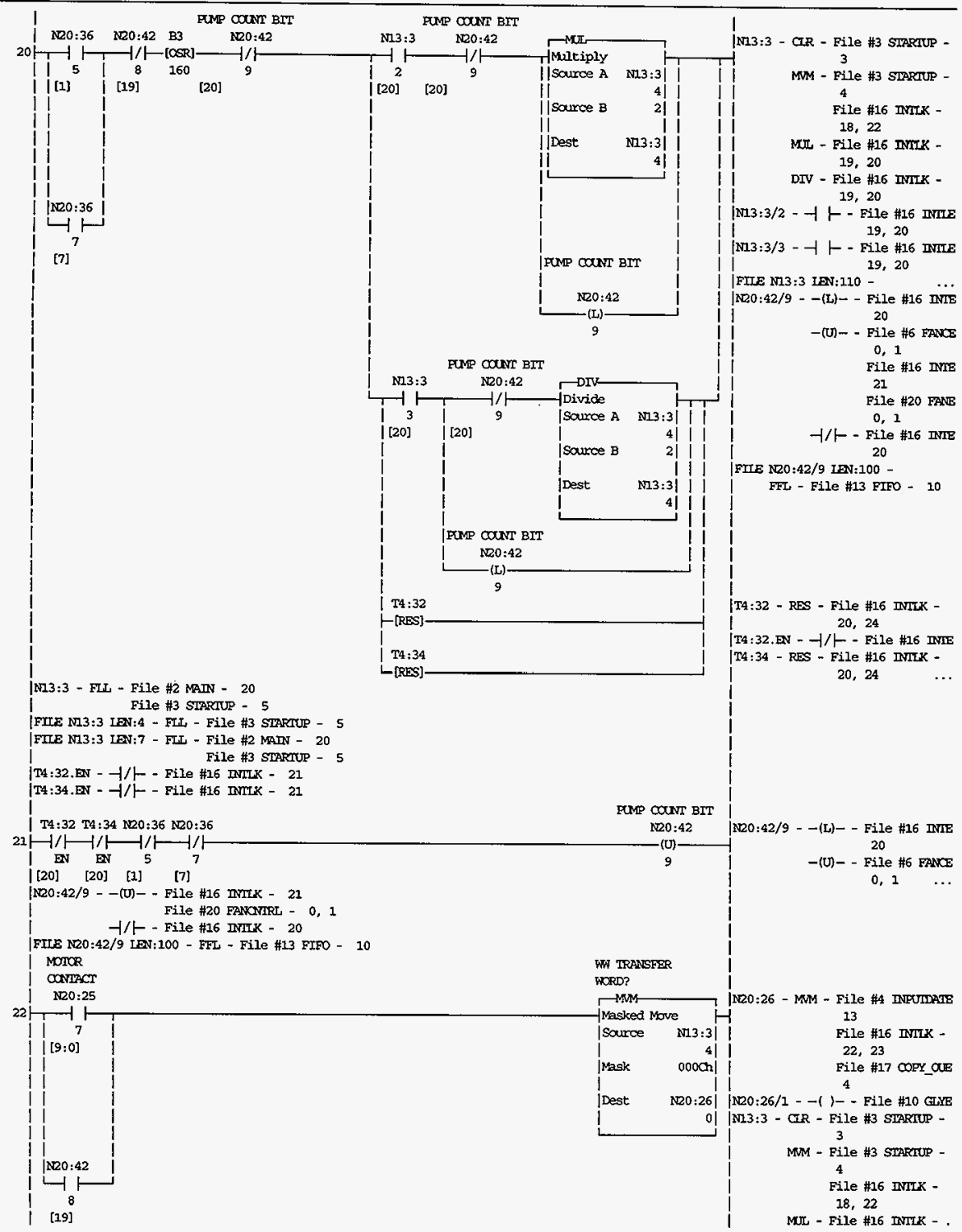


|N20:26/1 - - ( )- - File \#10 GrootgMP - I Page:00098

|N20:26/4 - - ( ) - File \#16 DNTK - 37

|N20:26/5 - - ( ) - File \#16 ANILX - 38

|N0:26/6 - -1/ - File \#16 INIIK - 12

|N20:26/7 - -|/ - File \#16 INIIK - 12

N20:26/8 - - 1 - File \#16 INTLK - 16

N20:26/10 - ᄀ ト - File \#16 ININK - 19

N20:26/11 - - F - File \#16 INITK - 19

FIIE NE0:26 IEN:100 - FFL - File \#13 FIFO - 10

|FIIE N20:26 IEV:30 - FFL - FIIe \#18 FIFOBUFFR - 1

|FILE N20:26 IEN:30 - FFL - FIIE \#18 FIFCBUFFER - 1

|FIIE N20:26 IEN:30 - FFL - File \#18 FIFOEUFFER - 1

ND3:3 - MU - File \#16 TNILK - 19, 20

f DIV - File \#16 INIK - 19, 20

|NL3:3/2 - - F - File \#16 nNTL - 19, 20

|N13:3/3 - - f - File \#16 INILX - 19, 20

|FIE N13:3 LEN:110 - FLL - File \#2 MAIN - 20

| File \#3 STAFTUP - 5

|FIIE N13:3 LEN:4 - FUL - File \#3 STARTUP - 5

|FILE N13:3 IEN:7 - FLL - File \#2 MADN - 20

1 MOICR File \#3 SIARTUP - 5

CONIACT

NDO:42 N20:25

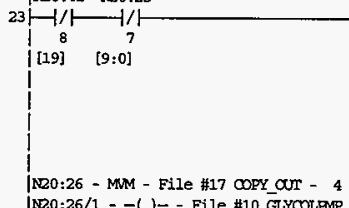

|NE0:26/1 - - ( ) - File \#10 GYOOLAMP - 1

|N20:26/4 - -( ) - File \#16 INIEK - 37

N20:26/5 - - ( ) - File \#16 INILK - 38

NE0:26/6 - - // - File \#16 INILK - 12

|N20:26/7 - - / - File \#16 INILK - 12

Ne0:26/8 - - - - File \#16 INILK - 16

N20:26/10 - - ト File \#16 MNTK - 19

N20:26/11 - - F - File \#16 INIL - 19

|FDE N20:26 LON:100 - FFL - File \#13 FIFO - 10

|FIIE NR0:26 LEN:30 - FFL - File \#18 FIFOEUFFER - 1

|FIE RR0:26 LEN:30 - FFL - File \#18 FIFOBUFFER - 1

|FIE NR0:26 LEN:30 - FFL - File \#18 FIFCEUFFER - 1

N13:0 - NOF - File \#3 SIRETUP - 2

FLL - File \#2 MATN - 20

File \#3 SIRATU? - 5

MM - File \#3 STRRIJP - 11

File \#7 MESGVIEN - 1

File \#16 INILK - 18, 23

N13:0/0 - -1 - File \#13 FIFO - 0, 1, 2, 3

1 File \#14 CONIER - 0

|FIIE M3:0 IEN:110 - FLI, File \#2 MAIN - 20

File \#3 SIAPTUP - 5

|FTIE M3 0 IEN:3 - FLU - File \#3 STARIOP - 5

|FIIE N13:0 LEN:4 - FLL - File \#3 SIARTUP - 5

|FIE M3:0 IEN: 7 - FLL - File \#2 MAIN - 20

File \#3 STARIUP - 5

|MOIOR

|CONIACT

$\mathrm{N} 20: 25$

T4:34.EN - - / - File \#16 INITK - 21

$T 4: 32$ T T4:32 - RES - File \#16 INILK -

20, 24

WOeD?

$\rightarrow \mathrm{MW}$

Masked Move

|source

N23:01

N20:26 - MM - File \#4 INPUIDATB 13

Nask $000 \mathrm{~h} \mid$ |N13:0 - CIR - File \#2 ManN - 0

| | File \#3 STRRTIP -

Dest $\quad$ NoO:26| | 1

NOT - File \#2 MAN - 1

File \#3 SIARIUP - 


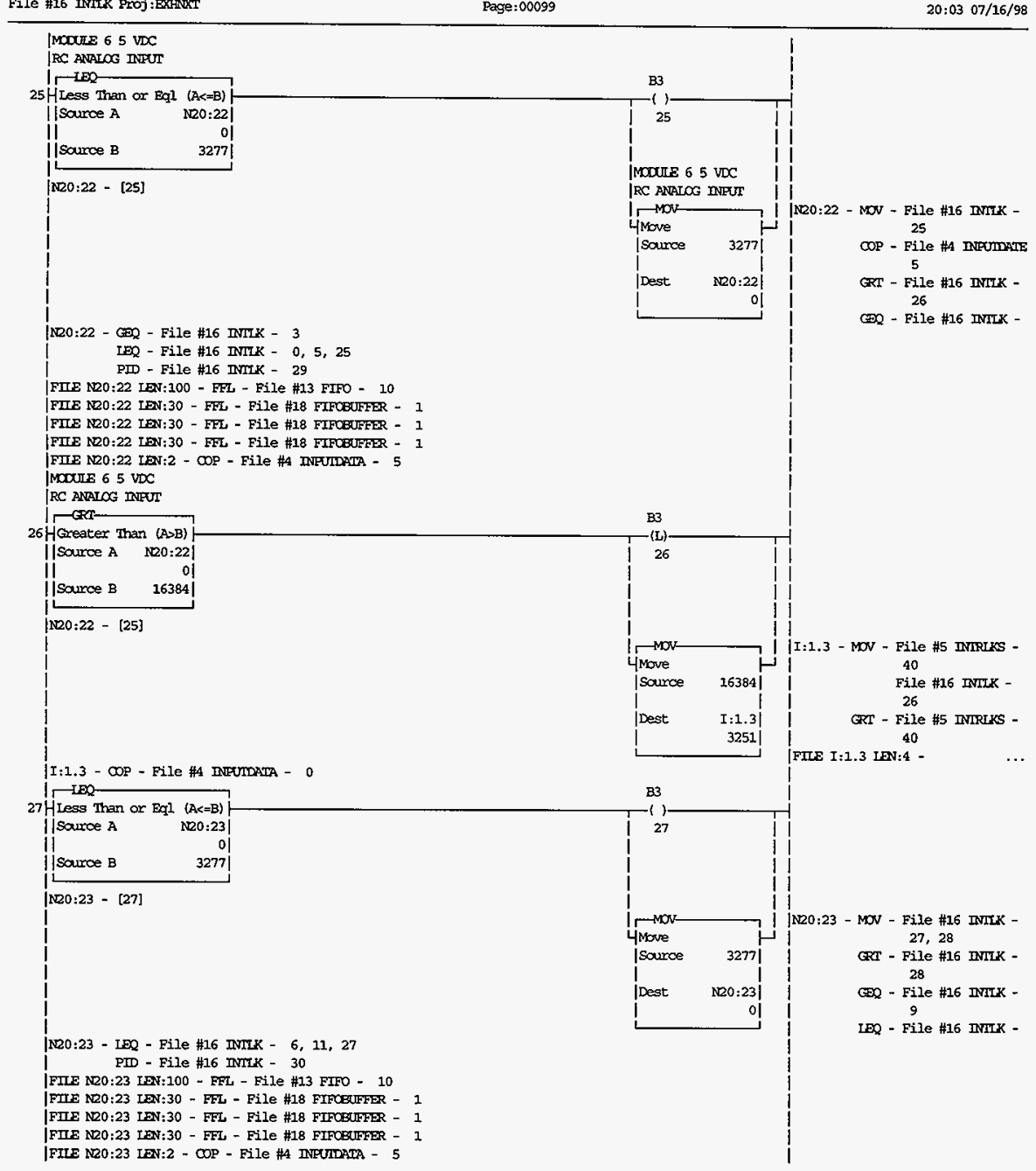




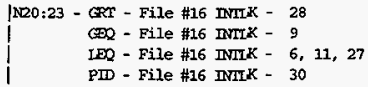

Time bode............. $0 \mathrm{TM}$

Auto/Marmal...........: 0 AM

Control mode. ............: 0 OM

output Liniting Enable...: o OL

Reset and Gain Range.....: 1 RG

Scale Setpoint Flag......: 0 SC

Loop Update Time Too Fast: 0 IF

Derivative (rate) Acticn.: O DA

Set when Error is in DE. .: $0 \mathrm{DB}$

output Alarm, Upper Limit: $0 \mathrm{UL}$

output Alam, Lower Limit: $0 \mathrm{LL}$

Setpoint out of Range.... : 0 SP

Process var out of Rarye. : 0 PV

PII Done............. 0 IN

PID Enabled. ..........: 0 EN

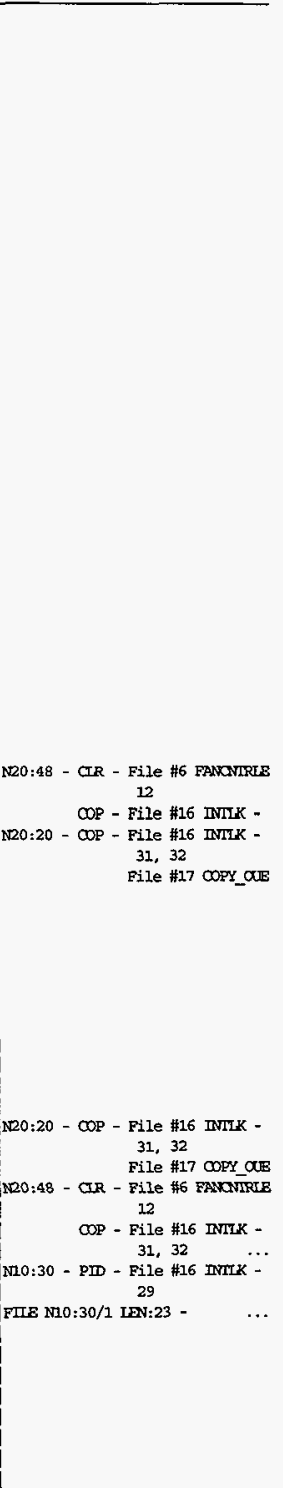


[N20:48 - FFL - File \#13 FIFO - 10

|FIIE N20:48 IEN:1 - COP - File \#16 INFK - 31, 32 N10:30/1 - PID - File \#16 INIIK - 29

3

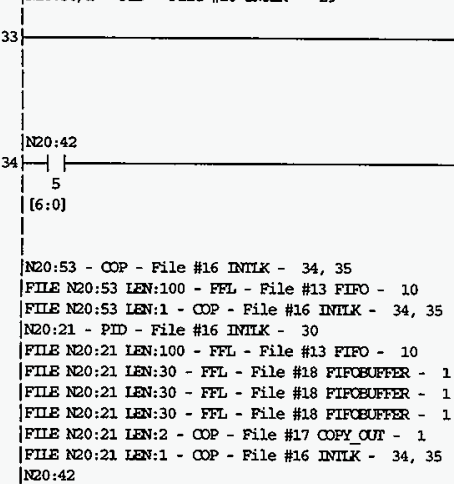

35

$\mid$\begin{tabular}{c|c}
5 \\
$\mid$ \\
$\mid$
\end{tabular}

|N20:21 - PID - File \#16 INILK - 30

|FILE N20:21 LDN:100 - FFL - File \#13 FIFO - 10

|FIIE NR0:21 LON:30 - FFL - File \#18 FIFCEUFFER - 1

|FIIE N20:21 IFN:30 - FFL - File \#18 FIFCBUFFR - 1

FIIE N20:21 LEN:30 - FFL - File \#18 FIFCBUFFER - 1

|FIIE N20:21 IEN:2 - COP - Pile \#17 OOPY OUT - 1

|FILE N20:21 LEN:1 - DO - File \#16 RIIX - 34, 35

|N20:53 - FFL - File \#13 FIFO - 10

|FIIE N20:53 LEN:1 - OOP - File \#16 INILK - 34, 35

N10:60/1 - PID - File \#16 INILK - 30

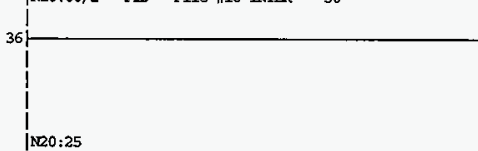

$37-1$

| $\begin{gathered}9 \\ {[4: 12]}\end{gathered}$

|N20:26 - MM - File \#17 COPY OTI - 4

|FIIE N20:26/4 InV:100 - FFL - File \#13 FIFO - 10

|FILE ND0:26/4 LDV:30 - EFL - File \#18 FIFCBUFFER - 1

|FIIE N20:26/4 LEN:30 - FFL - File \#18 FIFOBUIFFER - 1

|FIIE N20:26/4 LEN:30 - FFL - File \#18 FIFCEIFFER - 1 |N20:25

$38 \longmapsto 1$

10

| $[4: 12]$

|N20:26 - MM - File \#17 COHY Or - 4

|FIIE N20:26/5 LEV:100 - FFL - File \#13 FIFO - 10

|FIIE N20:26/5 LEN:30 - EFL - File \#18 FIFOBJJFER - 1

|FIIE ND0:26/5 IEN:30 - FFL - File \#18 FIFCERIFFER - 1

|FITE N20:26/5 IEN:30 - FFL - File \#18 FIFCEREFER - 1

(1)

39

C.... Copy File

|Samce fNo:49| |FILE N20:49 Iav:100.

|Dest \#N10:32| | FFL - File \#13 FIFO - 10

|Length 4]

$\longrightarrow$ COP

|source \#20:21|

|N20:53 - CIR - File \#6 FANONIRIE

12

Jest \#N20:53| |N20:21 - COP - File \#16 INIIX -

i| $\quad 34,35$

1 PID - File \#16 INILK - 
Portable 1000 CM Exhauster Program STC-500 Ladder Iisting

[END]-1

HNF-2934

Rev 0

A-103 


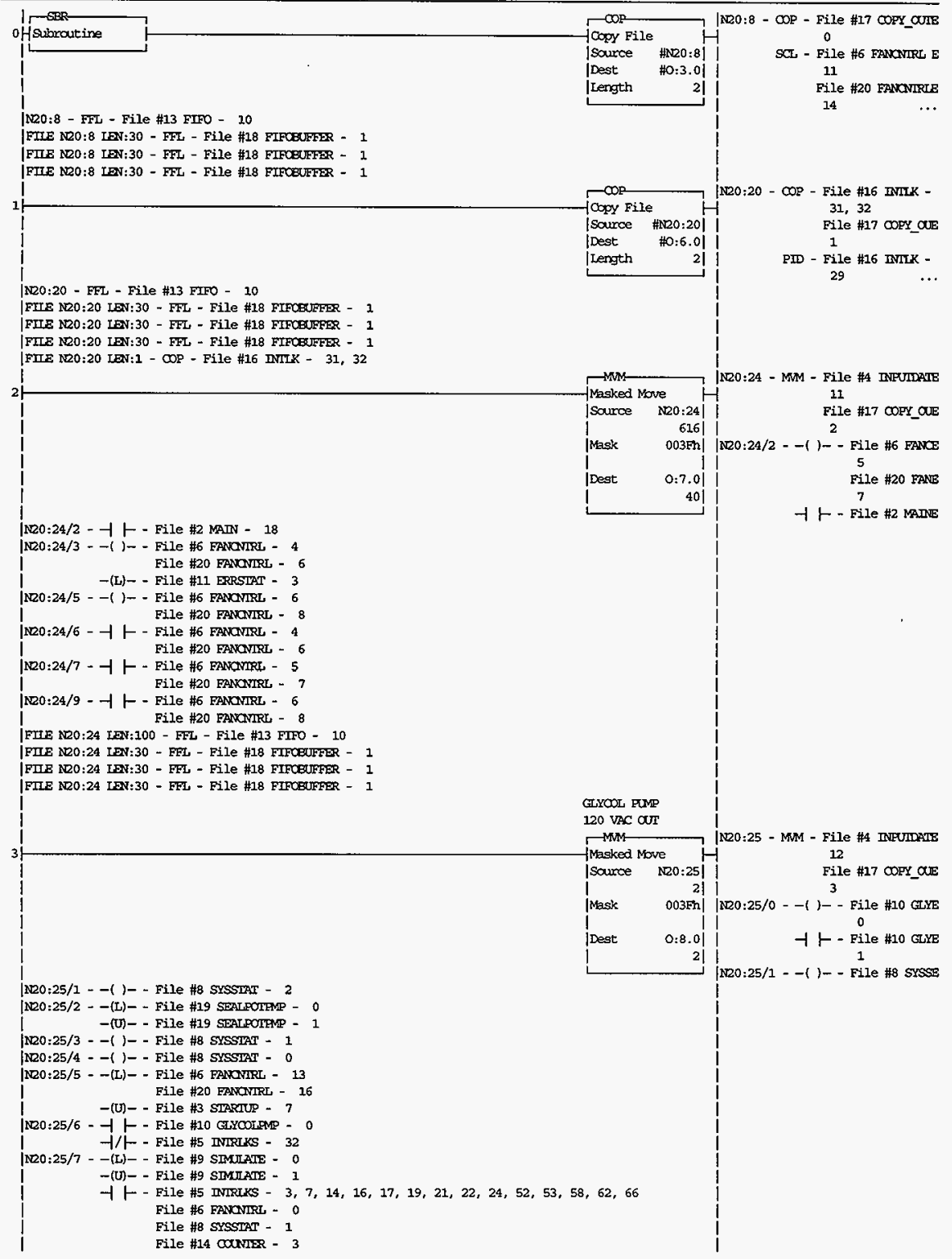

HNF-2934 


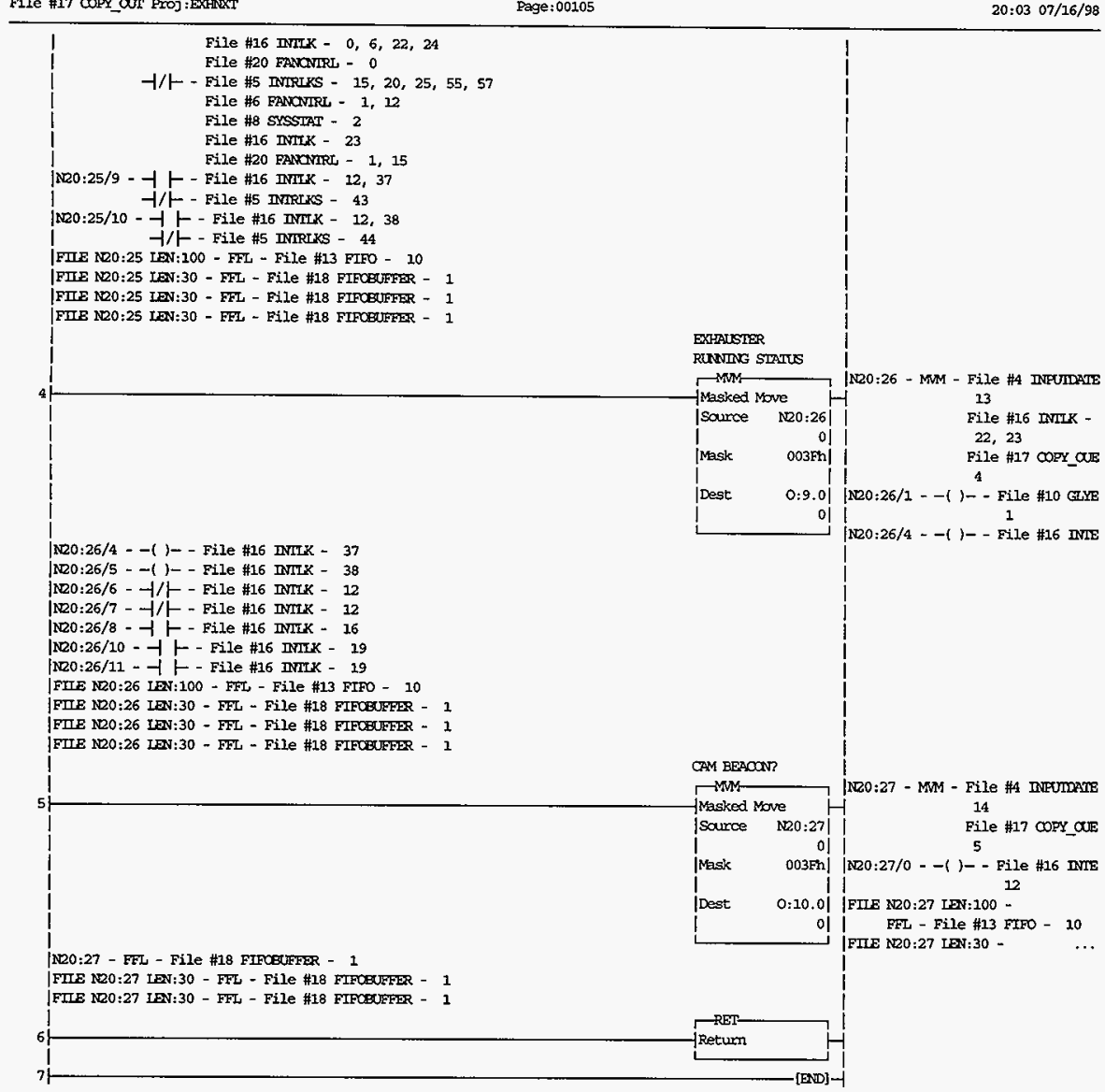


ITIS SUB FINS ALY RHBN THE FAN IS ON (B3/34)

$$
1
$$

FIFO LOAD TIMER

rTaN

Timer

Time Rase

irreset

ircoum

0.01
100

L
1 T

T4:37 - TON - File \#18 FIFOBUFFE 0

RES - File \#18 FIFCEUERE

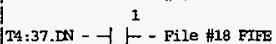
I

1

HNF-2934

Rev 0

A-106 


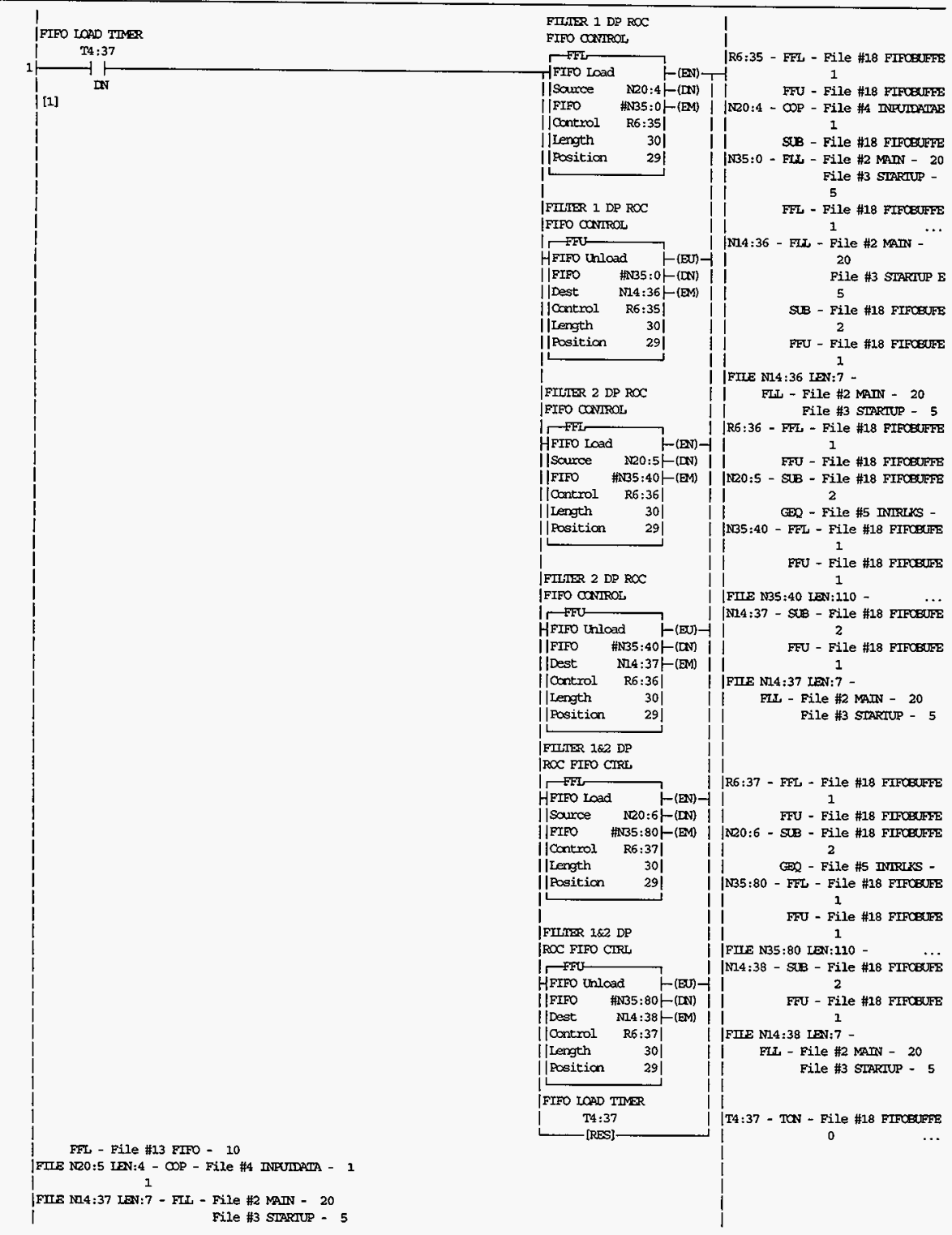


1

FIIE R6:37 LEN;128 - FFU - File \#13 FIFO - 5

|FILE R6:37 IDN:127 - FFU - File \#13 FIFO - 7

|FIIE R6:37 IDN:127 - FFU - File \#13 FIFO - 9

|FIIE R6:37 LDN:100 - FFU - File \#13 FIEO - 11

FIIE R6:37 LEN:128 - FFU - File \#13 FIFO - 13

|FILE R6:37 IEN:127 - FFU - File \#13 FIFO - 15

FIIE R6:37 IAN:127 - FVU - File \#13 FIFO - 17

|FIIE R6:37 IDN:100 - FNU - File \#13 FIFO - 19

[FIIE R6:37 LFN:128 - FFU - File \#13 FIFO - 21

|FIIE R6:37 LDN:127 - FFU - File \#13 FIFO - 23

|FILE R6:37 LPN:127 - FFU - File \#13 FIPO - 25

|FLLE R6:37 LEN:100 - FFU - File \#13 FIFO - 27

|FIIE R6:37 IEN:128 - FRU - File \#13 FIFO - 29

|FTE R6:37 IEN:127 - FFU - File \#13 FIFO - 37

|FIIE R6:37 IEN:127 - FFU - File \#13 FIFO - 33

FIIE R6:37 IEN:100 - FFU - File \#13 FIFO - 35

|FIIE R6:37 IEN:128 - FFU - File \#13 FIFO - 37 |FIIE R6:37 IEN:127 - FFU - File \#13 FIFO - 39 |FILE R6:37 LEN:127 - FEU - File \#13 FIFO - 41 |FILE R6:37 IEN:100 - FUU - File \#13 FIFO - 43 |FIIE R6:37 IAN:128 - FEU - File \#13 FIFO - 45 |FIE R6:37 IEN:127 - FRU - File \#13 FIFO - 47 |FIEE R6:37 IDN:127 - FEU - File \#13 FIFO - 49 |FILE R6:37 IEN:100 - FEU - File \#13 FIPO - 51 |FIIE R6:37 IEN:30 - FFU - File \#18 FIFCEUFFR - 1 FIIE R6:37 IEN:30 - FFU - File \#18 FIFCBUFFER - 1 |FIIE R6:37 IEN:30 - FFU - File \#18 FIFCEUFFER - 1

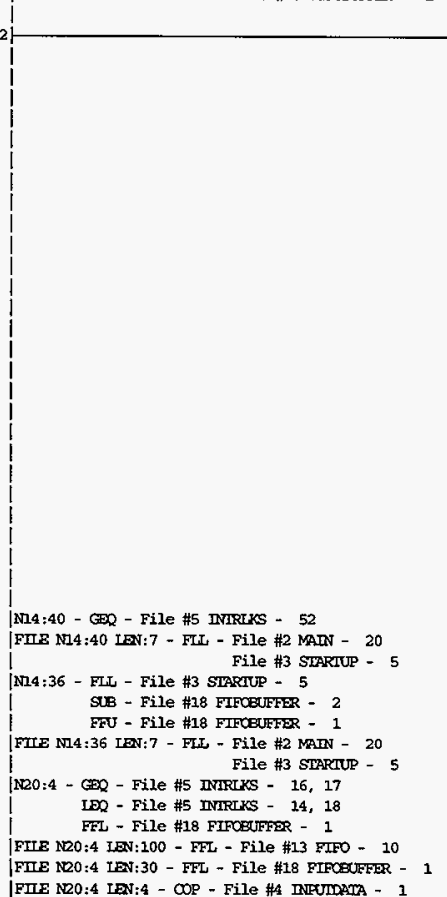

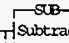

isouract

11

| |sou

II

IIDest

11

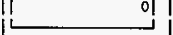

SUB

Hsubtract

||source A N14:37|

II

11

I

IIDest $\quad$ 3291| |

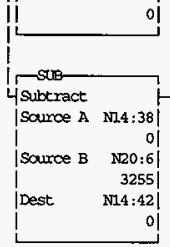

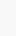

IN14 :40 - STE - File \#18 FIFCBUES 2

GOS - File \#5 INRTLS E

0] | N14:36 - FL - File \#2 MAN -

20

File \#3 STARIUP $\mathrm{E}$

4:40| | |N20:4 - 0 - File \#4 INTUmaIAE

||$^{1}$

I SUB - File \#18 FIFOBUEFE |N14:41 - SUB - File \#18 FIFOBUHE -1 2 2

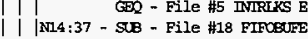
FFU - File \#18 FIFCBUFE
iNo:5 - SUB - File \#18 FIFCBUFF I 2 I GPQ - File \#5 INIRLKS 21,2 |FIE N14:42 LEN:7 FLL - File \#2 MAIN - 20 File \#3 STARTUP - 5 M14:38 - SUB - File \#18 FIFOEUFE I 2

I |N20:6 - SUB - File \#18 FIFCBUFFE 2 GEQ - File \#5 INIRLKS - 
|N14:41 - File \#5 INIRIKS - 53

|FUE ND4:41 LEN:7 - FLL - File \#2 MADN - 20 File \#3 STARTUP - 5

|M14:37 - FFU - File \#18 FIFOBUIFER - 1

|FIIE N14:37 LEN:7 - FLL - File \#2 MANN - 20

I File \#3 stakiur - 5

INO:5 - INQ - Eile \#5 INIRUKS - 19, 23

1 FFL - File \#18 FIFOEUFFE - 1

|FIIE N20:5 LEN:100 - FFL - File \#13 FIFO - 10

|FILE N20:5 LEV:30 - FFL - File \#18 FLFOEUFFER - 1

|FILE ND0:5 LEN:4 - COP - File \#4 INFUIDATA - 1

FIIE NQ0:5 IEN:30 - FFL - File \#18 FIFCEUFFER - 1

|N14:38 - FFU - File \#18 FIFCEUFEER - 1

|FIIE 2IL4:38 IEN:7 - ELU - Bile \$2 NAIN - 20

I File \#3 STARTuP - 5

IND0:6 - GD2 - File \#5 INIRIKS - 26

| IDR - File \#5 INIRLXS - 24

1 FFL - File \#18 FIFCESTFER - 1

PFIE N20:6 IEN:100 - FFL - File \#13 FIFO - 10

|FILE N20:6 IEN:30 - FFL - File \#18 FIFCBUFFER - 1

|FIE N20:6 IEN:4 - COP - File \#4 INEUIDATA - 1

|FIIE N20:6 IEN:30 - FFU - File \#18 FIFCRUEFER - 1

|FILE N20:6 IEN:30 - FFL - File \#18 FIFCBUEFER - 1

31 


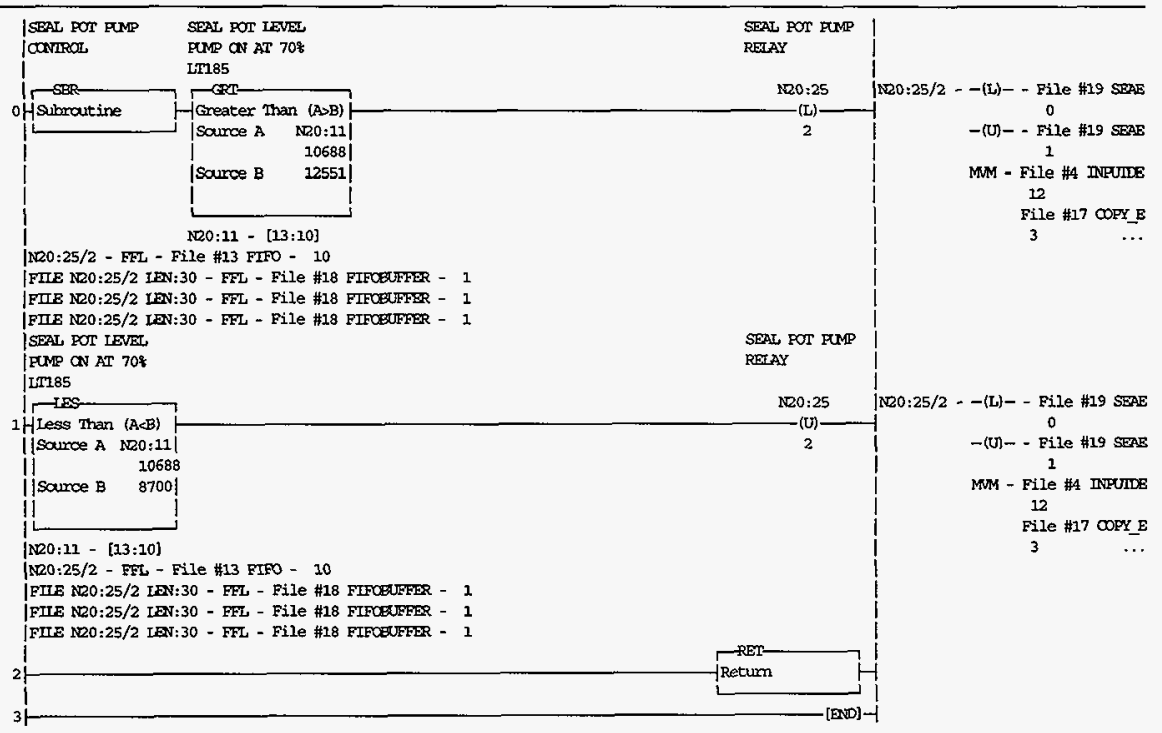




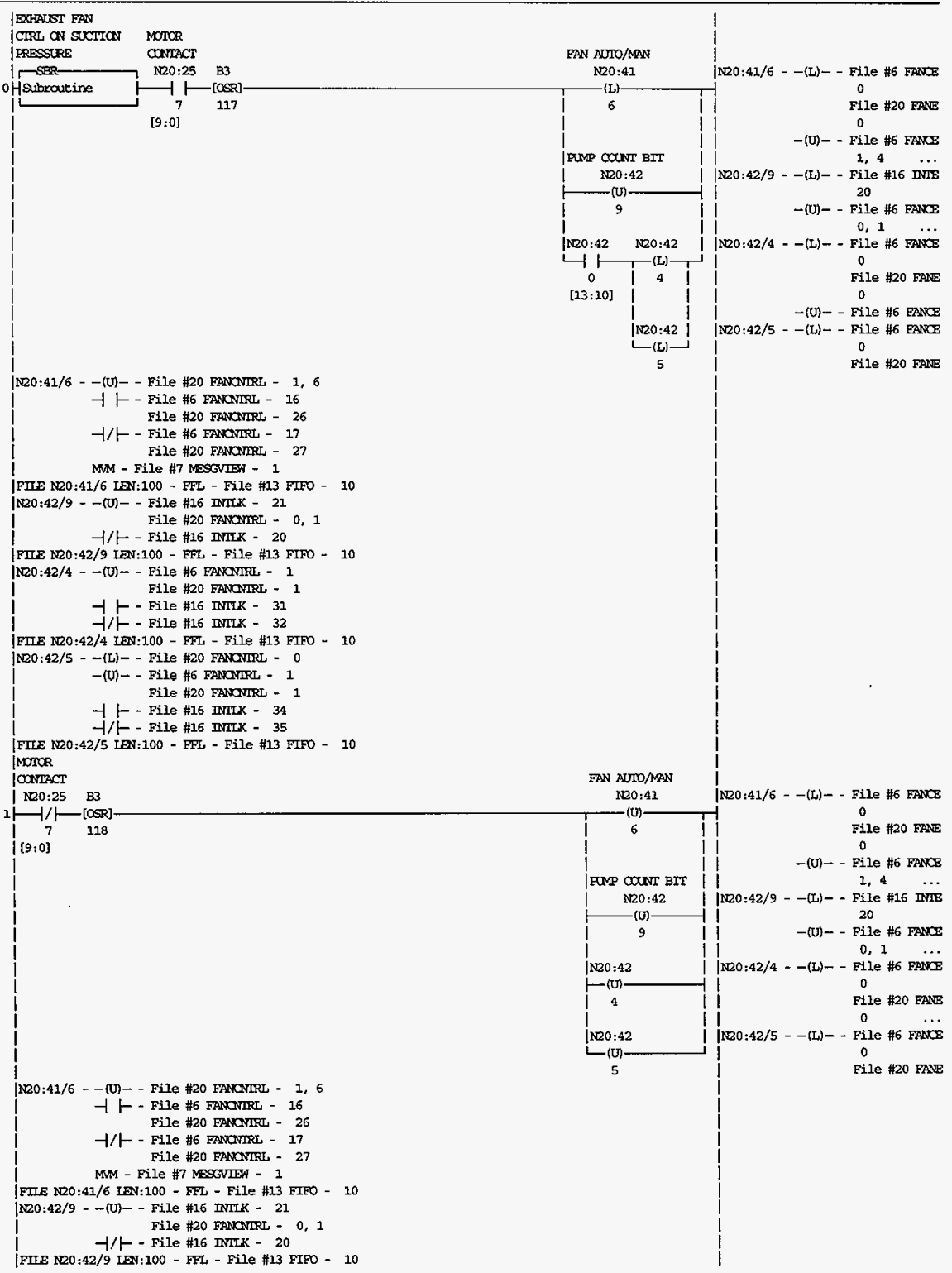

HNF-2934 


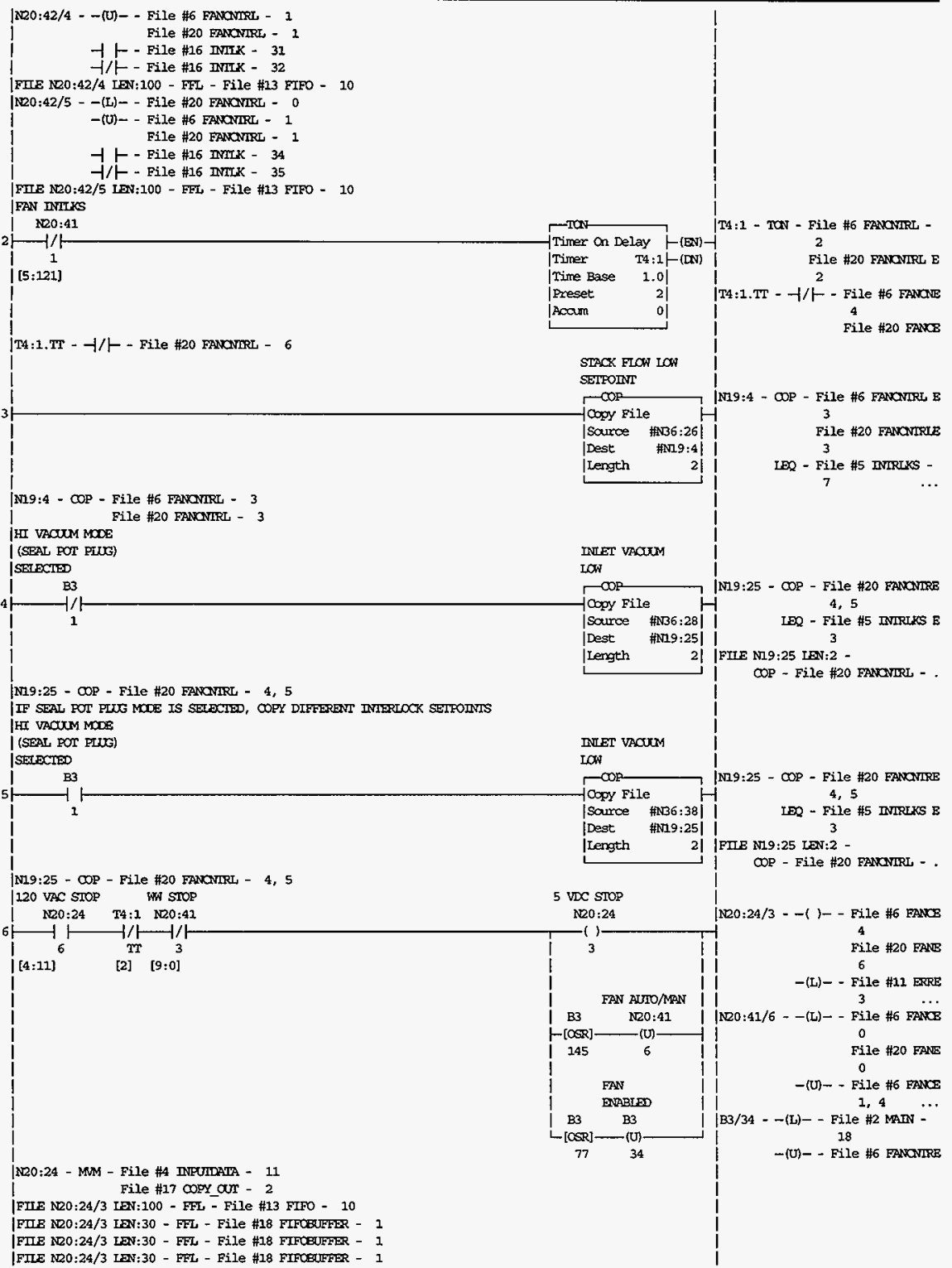




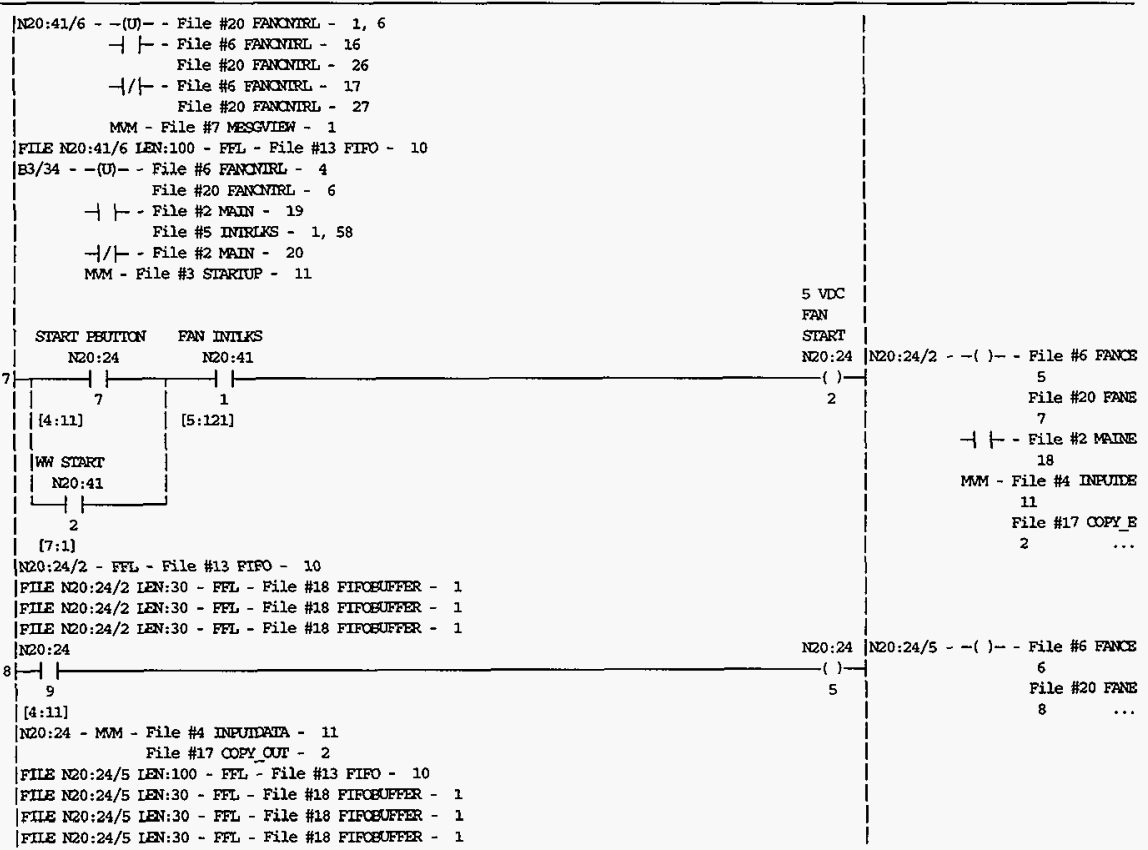




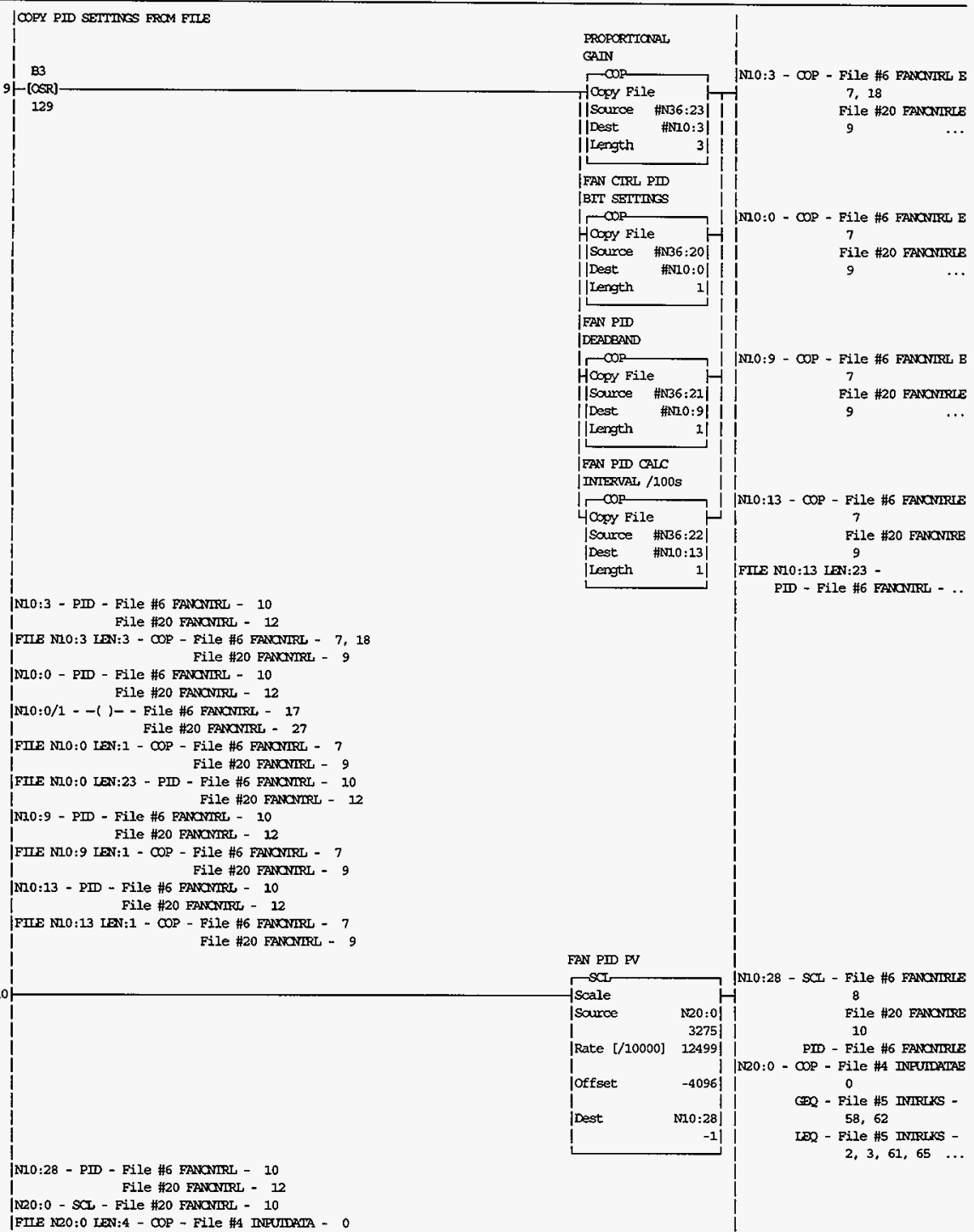




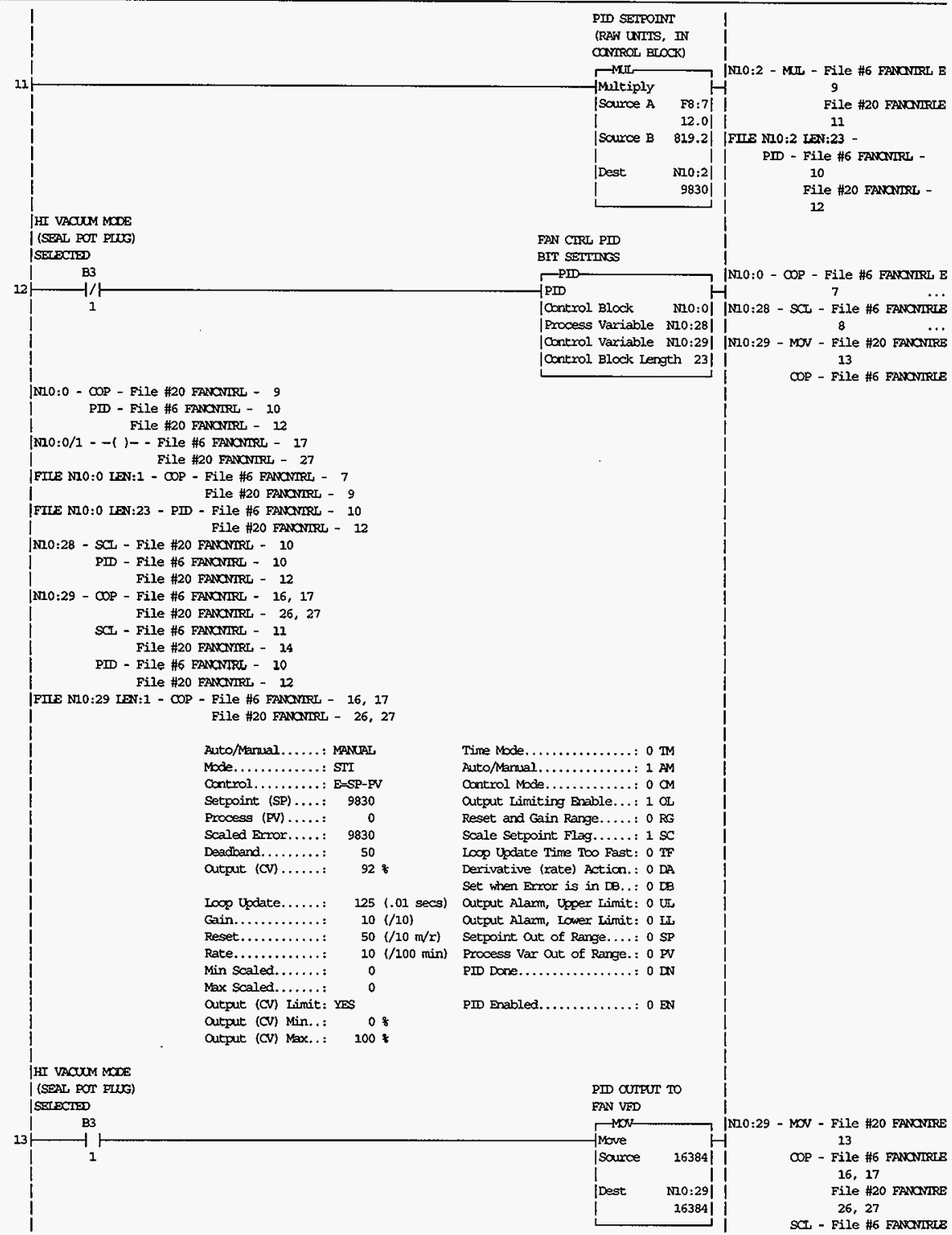




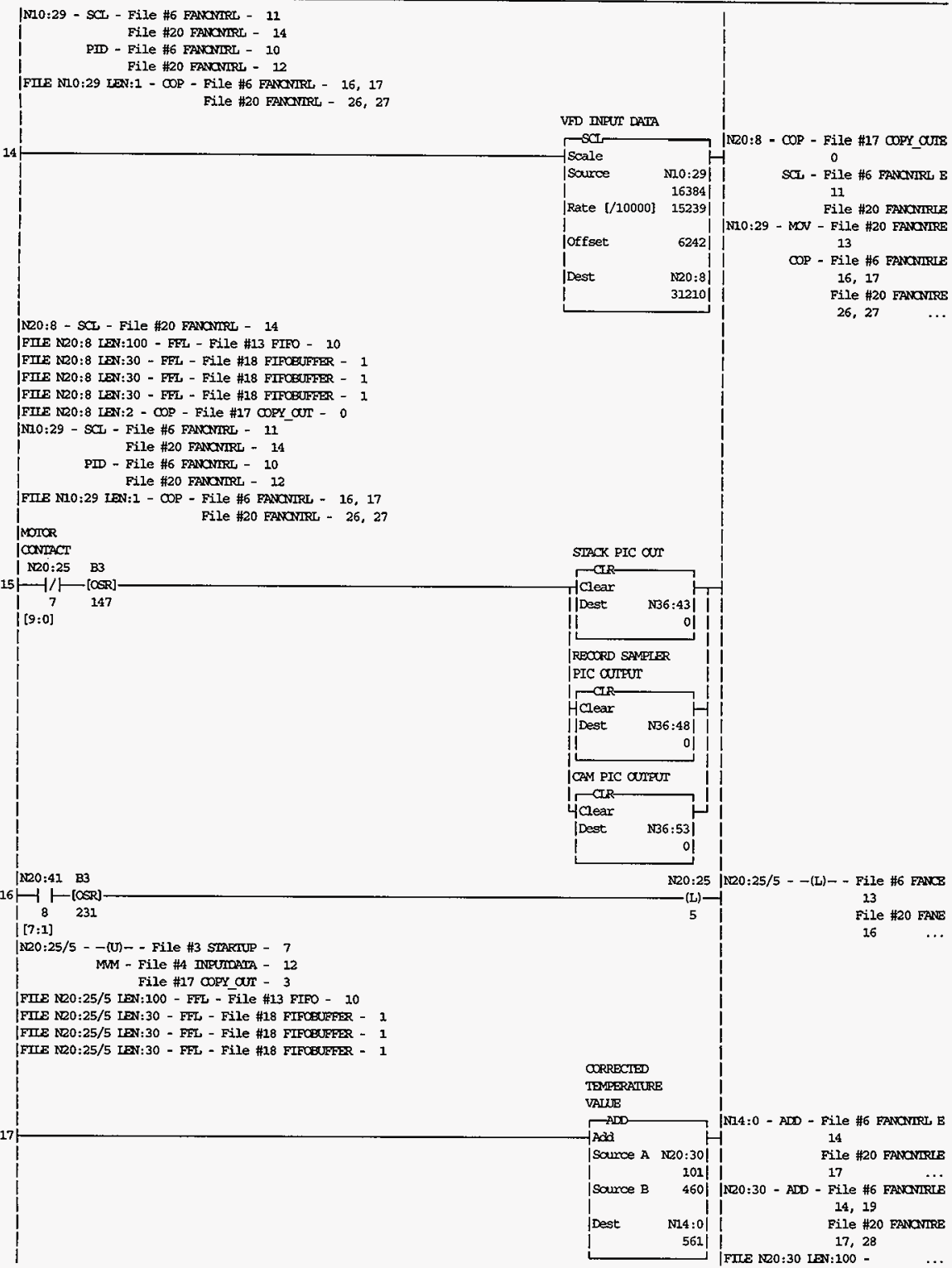




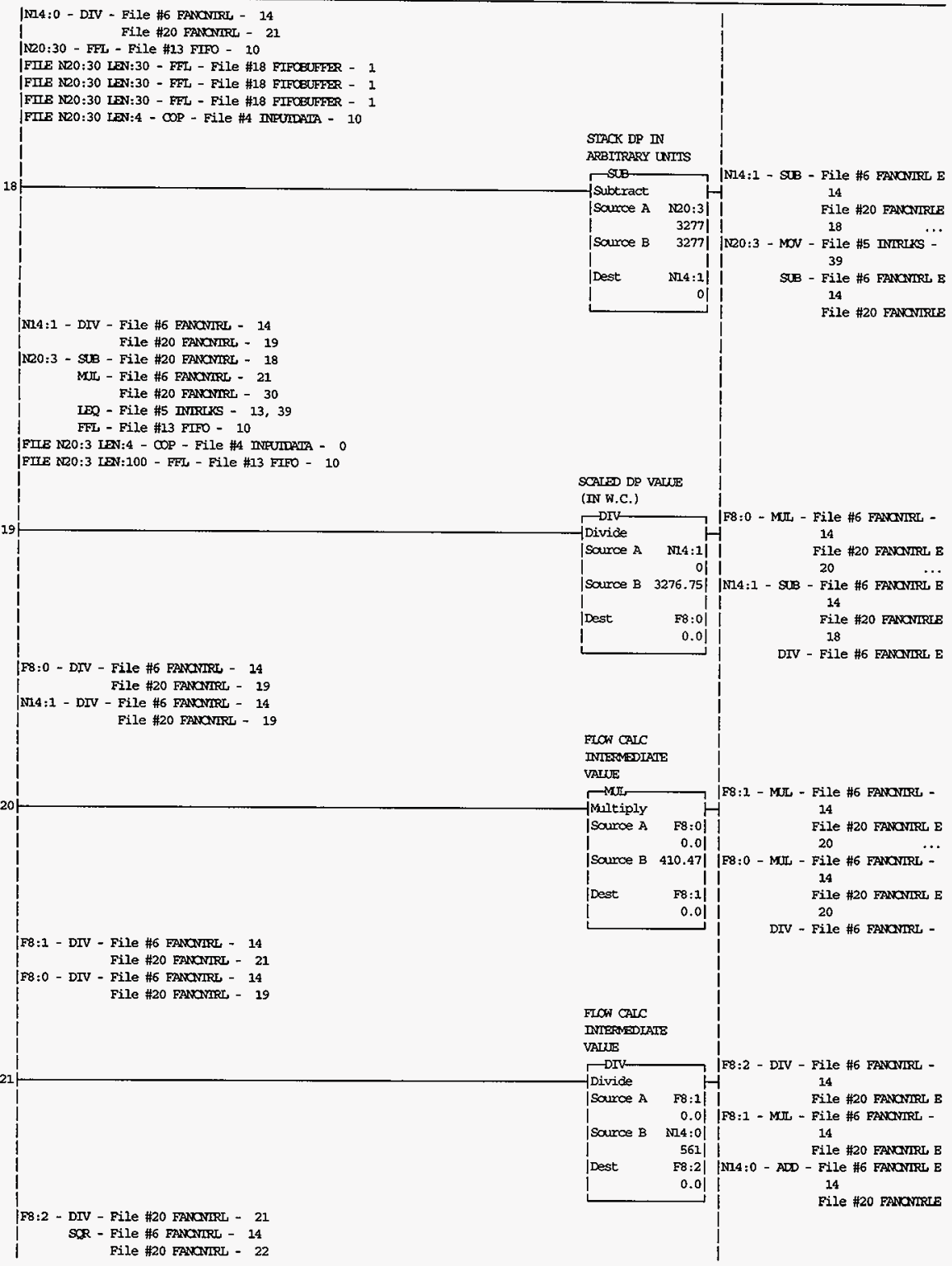




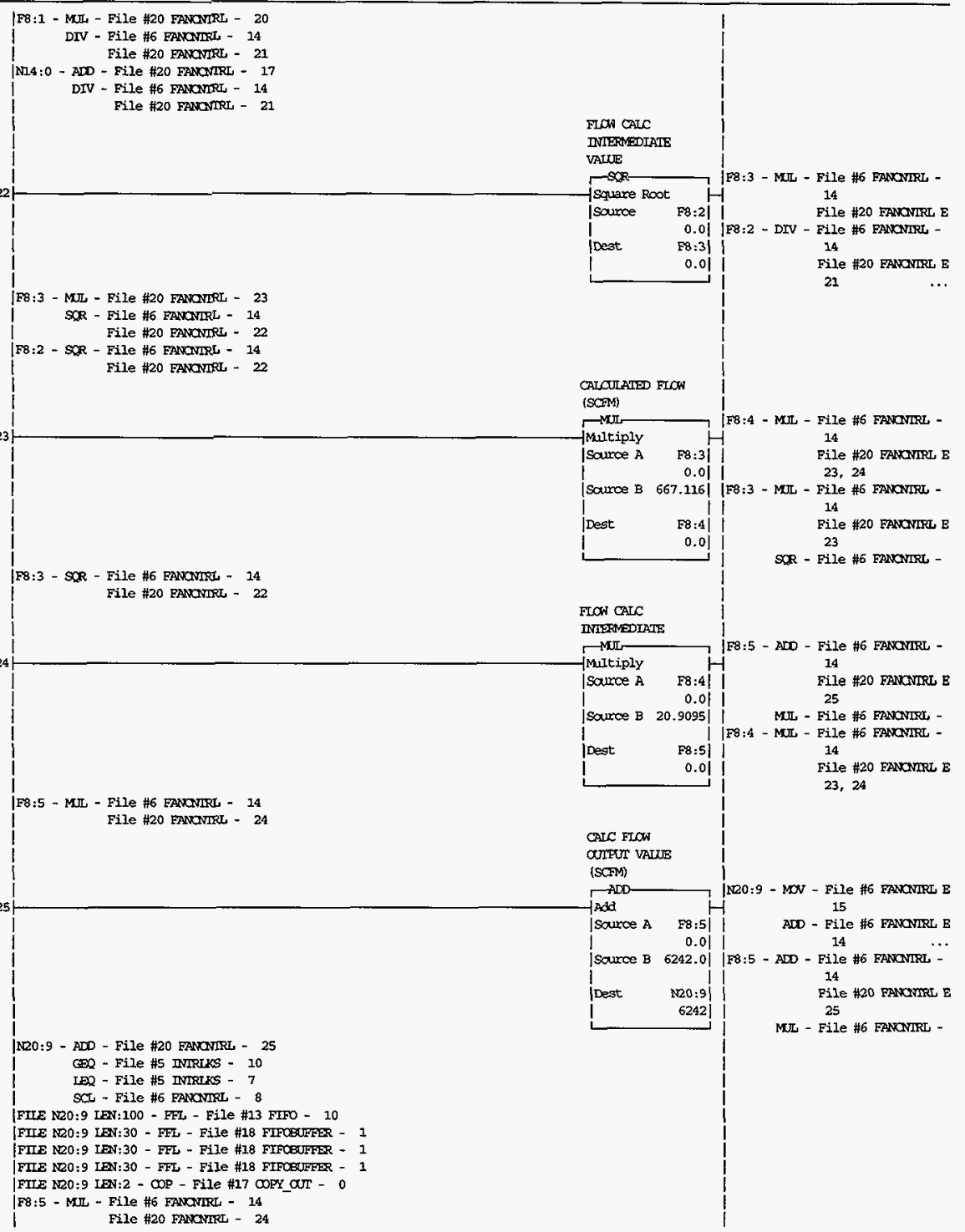




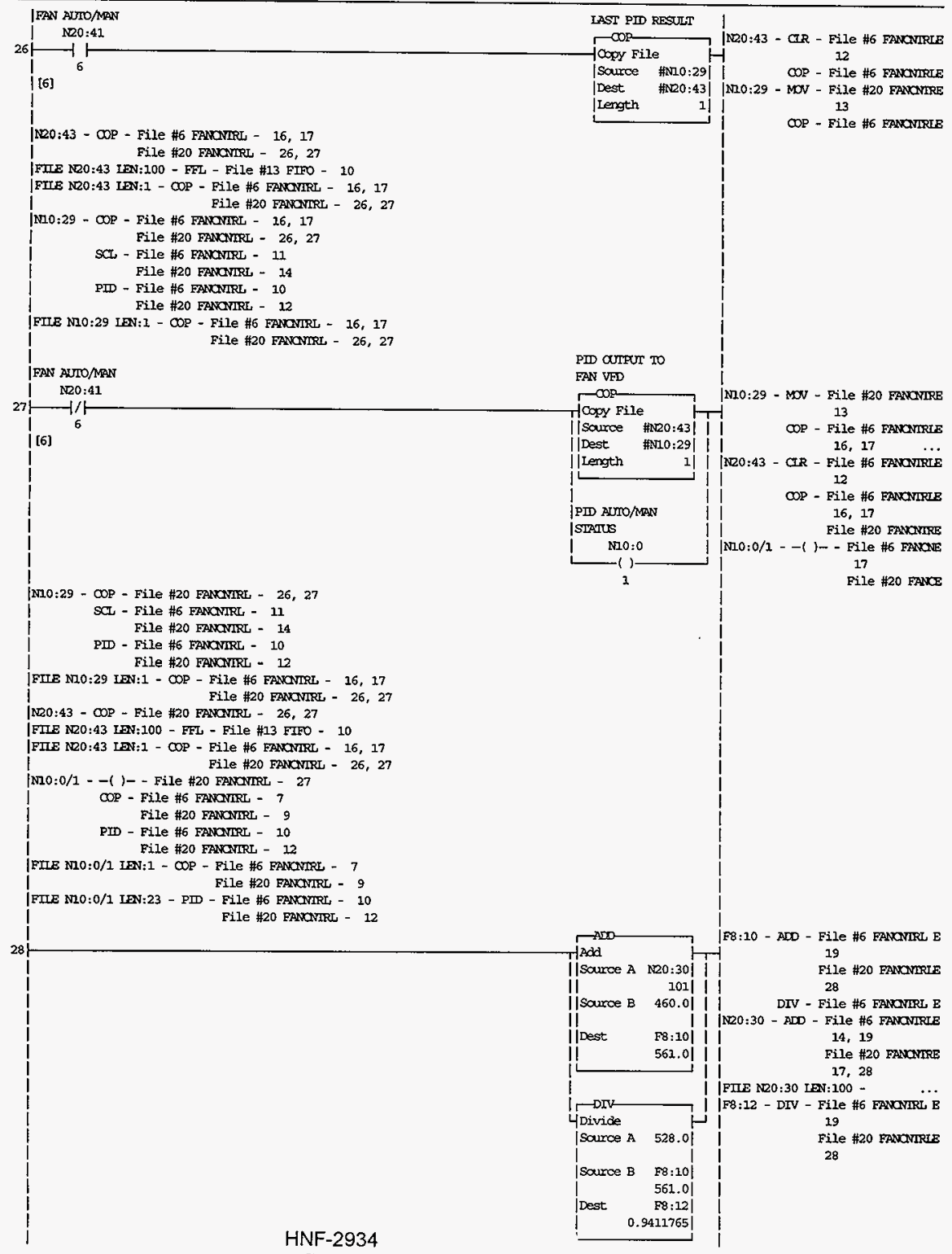

Rev 0 
|F8:10 - DTV - File \#6 FANONIRL - 19

File \#20 FavaNiRL - 28

|N20:30 - FFL - File \#13 FIFO - 10

|FILE NDO:30 IEN:30 - FFL - File \#18 FIFCBUFFER - 1

|FILE NOO:30 LEN:30 - FFL - File \#18 FIFCSUFFRR - I

|FIIE NDO:30 LEN:30 - FFL - File \#18 FIFOBOFFER - 1

FILE N20:30 IEN:4 - COP - File \#4 INETMATA - 10

29

F8:13 - DIV - File \#6 FANONIR - 20

| File \#20 FANONIRL - 29

1NO0:13 - FFL - File \#13 FIFO - 10

|FIIE N20:13 LEN:30 - FFL - File \#18 FIFCQTFFER - 1

|FIIE NE0:13 IEN:30 - FFL - File \#18 FIROBUFFER - 1

FIIE NCO:13 IEN:30 - FFL - File \#18 FIFCBUFFRR - 1

|FIIE N20:13 IEN:4 - OOP - File \#4 INFUMAM - 3

|F8:15 - DTV - File \#6 FANONIRL - 20

| File \#20 FANONIRL - 29
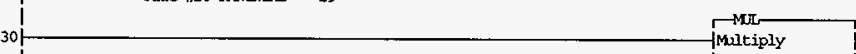

F8:19 - MUL - File \#6 FRMONIR E Multiply Iscunce A N20:31

21

100

II

I

0

[F8:13 - MU - File \#6 FananiR $\mathrm{B}$

$$
29
$$

DIV - File \#6 FANaNLr E NO0:13 - DIV - File \#6 FANOYIRIE

IDest

$F 8: 13$ | |

$0.01 \mid$

| FIIE N20:13 LEN:100

DIV _.

HDivide

11

II

II

$\left|\begin{array}{lr}\mid \text { Dest } & F 8: 15 \mid \\ & 0.01\end{array}\right| \begin{gathered}\mid \\ \text { l }\end{gathered}$

$|\Gamma-\mathrm{MIL}| \mid \mathrm{Fr}: 16$ - SUB - File \#6 FANONIR E

Hultiply

||source B F8:15| |

II

$\|$ in

11

I Homberact

I

Source B

$58: 16$

|Dest

$8: 17$

1.0

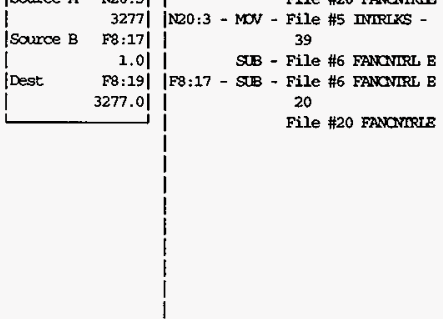

FIIE NDO:3 IBN:4 - COP - File \#4 INFUIDATA - 0

|FIIE N20:3 LEN:100 - FFL - File \#13 FIFO - 10 


F8:17 - SAB - File \#20 FANONIRL - 29
MII - File \#6 FANCNIRL - 21
File \#20 FANONIRL - 30

HNF-2934

Rev 0

A-12† 
WARNING : DESCRIPTION XREF REPORT WILL ONLY PRINT ADDRESSES THAT HAVE DESCRIPTIONS ENTERED INTO THE DATA BASE AND ARE USED IN THE LADDER PROGRAM!

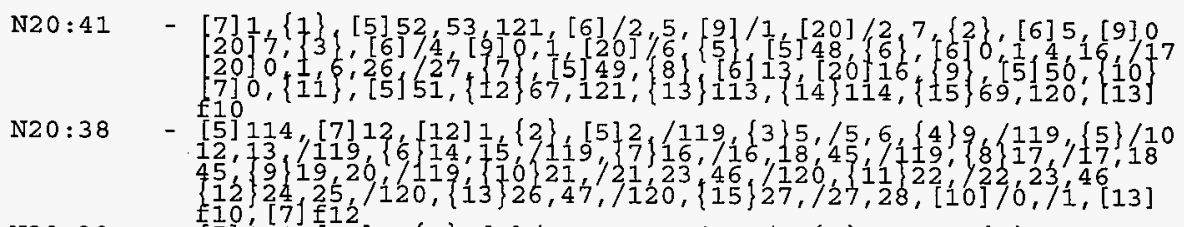

$\mathrm{N} 20: 39-[5] \frac{1}{3} 14,[12] 2,\{0\},[5] / 29,30,31,[10]\{1,\{1\},[5] 32,\{2\} 33, / 33,34$

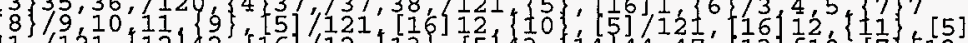

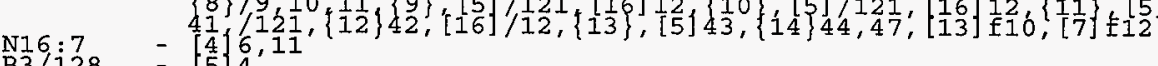

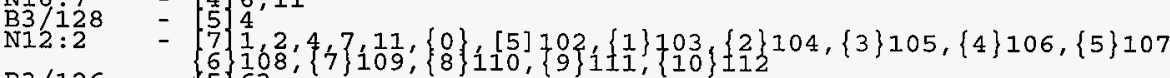

B3/126

N20:38/2

N16:10

N16:9

N16:8

$\mathrm{N} 20: 40$

B3/129

$-5,63$

$5 .\{2, / 119, w 114, w[7] w 12, w[12] w 1,[13] w f 10,[7] w f 12$

$4.9,14$

$4.8,13$

- 11,12

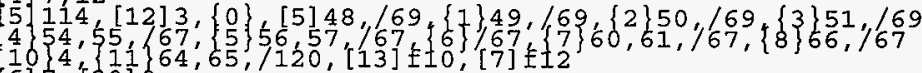

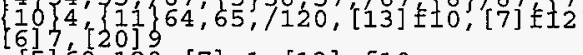

N20:41/15- [5]69,120, [7] w1, [13] wf 10

N20:42/1 - [5] $48,49,50,51,7120,[13] w f 10$

N20:42/6 - $55120,121,[13\}$ wf 10

N20:38/13

N20:39/3

$\mathrm{B} 3 / 130$

$\mathrm{T} 4: 5$. DN

B $3 / 73$

C5: 0

N19:21

$\mathrm{B} 3: 2$

$\mathrm{T} 4: 4 . \mathrm{DN}$

B 3116

$\mathrm{B} 3 / 17$

B3 109

B3 1106

B $3 / 112$

B3 $/ 0$

$I: 9.0$

[5] $26,47, / 120, w 114, w[7] w 12, w[12] w 1,[13] w f 10,[7] w f 12$

$[5] 35,36,1 / 120$, w114, w [12] w2, '[13] wf10, '7] wf12

- 5.530 .8

- 5.59

- 3$] 6,[15] 0,\{\mathrm{ACC}\} 1,\{\mathrm{CU}\} 0$

- 16]0

N20:24/6

$\mathrm{I}: 7.0$

3] $1 \frac{1}{6},\{32\},[5] 119,\{33\} 121,\{34\},[2] 18,19, / 20,[5] 1,58,[6] 4$

$-\frac{5}{5} \cdot 8,7$

- 5.55

- 519

$-5, \frac{1}{2} 4$

- OFIOW/VAC CTRL BIT (OFF=FLOW, ON=VAC)

$16,7,[5] / 2,3,58,62,119$

120
408 [4] 8 AC GE HIGH BETA

120 VAC STOP

$\mathrm{N} 20: 24 / 3$

$\mathrm{N} 20: 24 / 2$

$\mathrm{I}: 10.0$

[6] $4,[20] 6,[4] w 11, w[17] w 2,[13] w f 10,[18] w f 1, w f 1, w f 1$

[4] 6

5 VDC STOP

[6] $4,[11] 3,[20] 6,[4] w 11, w[17] w 2,[13] w f 10,[18] w f 1, w f 1, w f 1$ 1 3 [6] 5, [20]7, [4] w11, w [17] w2, [13] wf10, [18] wf1, wf1, wf1

N12:2/10 - ACK INLET VAC HI ALARM

[5] 112.217$] w 1, w 2, w 4, w 7, w 11$

N12:2/9 - ACK INLET VAĆ LO ÁARM

N13:0 - AL] $111,[7]$ W1, W2, W4 W7' W1

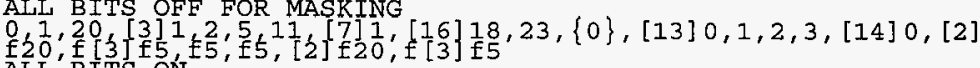

$\mathrm{N} 13: 1$

$1,[3] 2,4,10,[7] 1,5,6,7,[2] f 20, f[3] \pm 5, f 5, f 5,[2] £ 20, f[3] f 5$ 


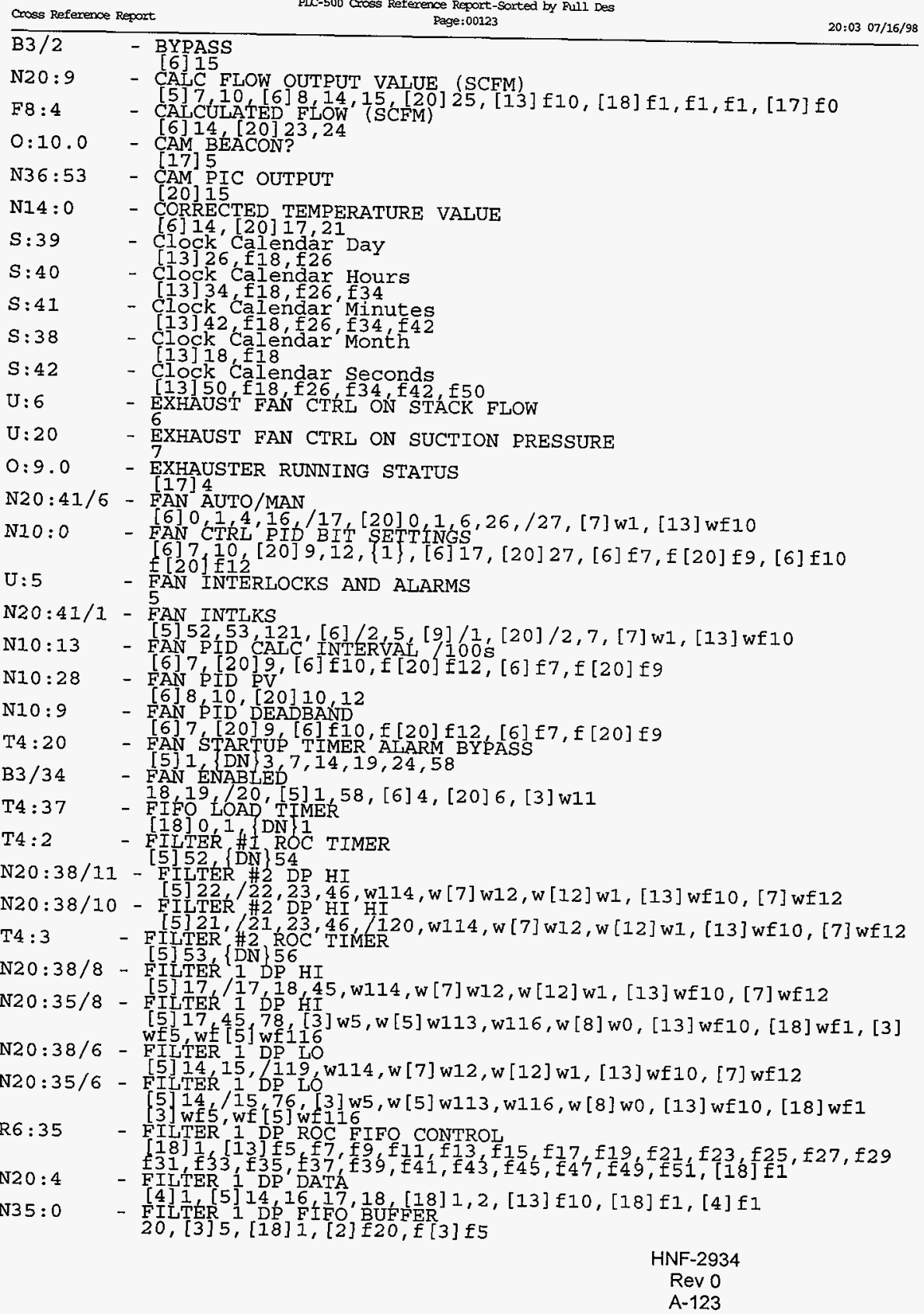




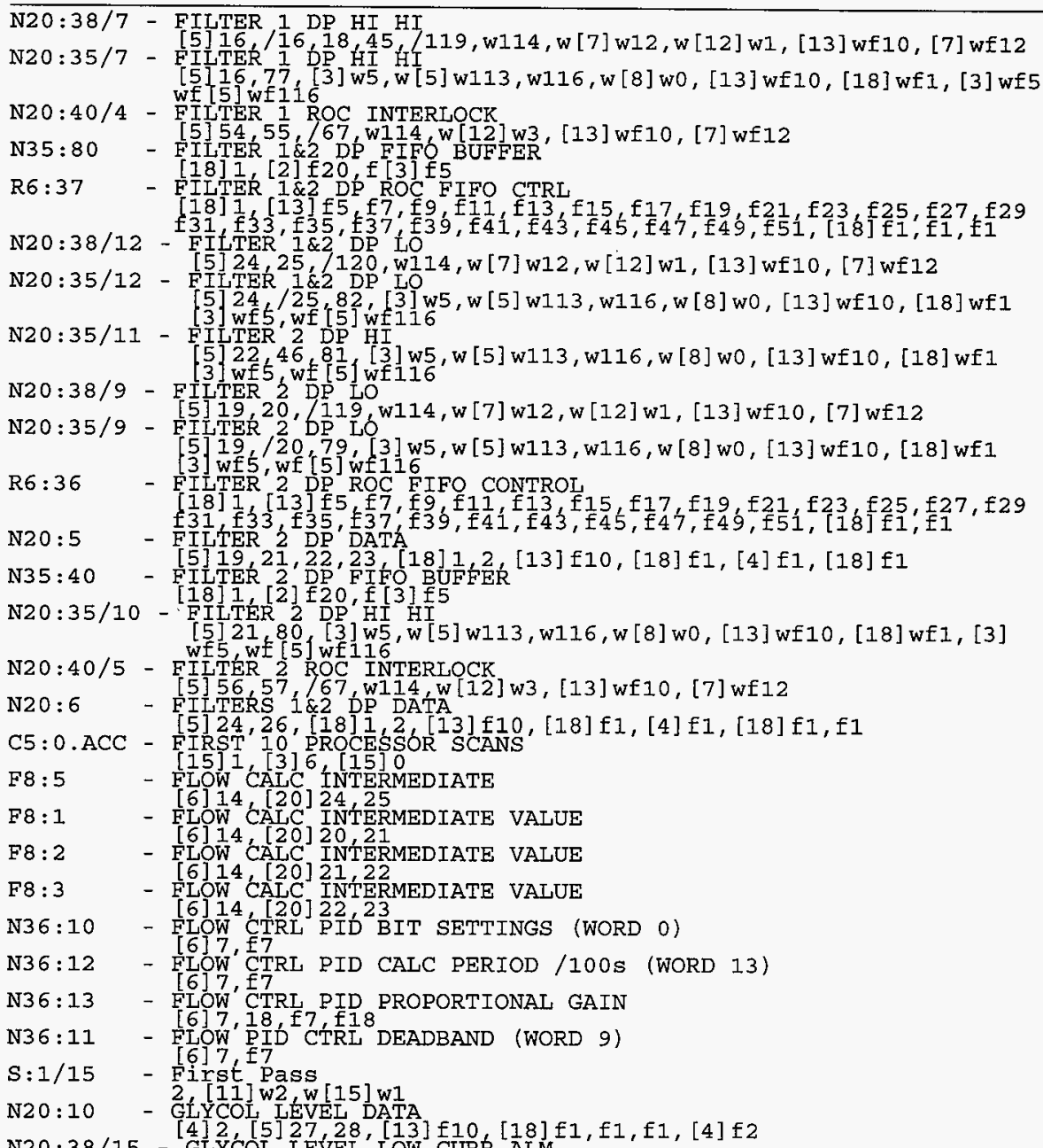




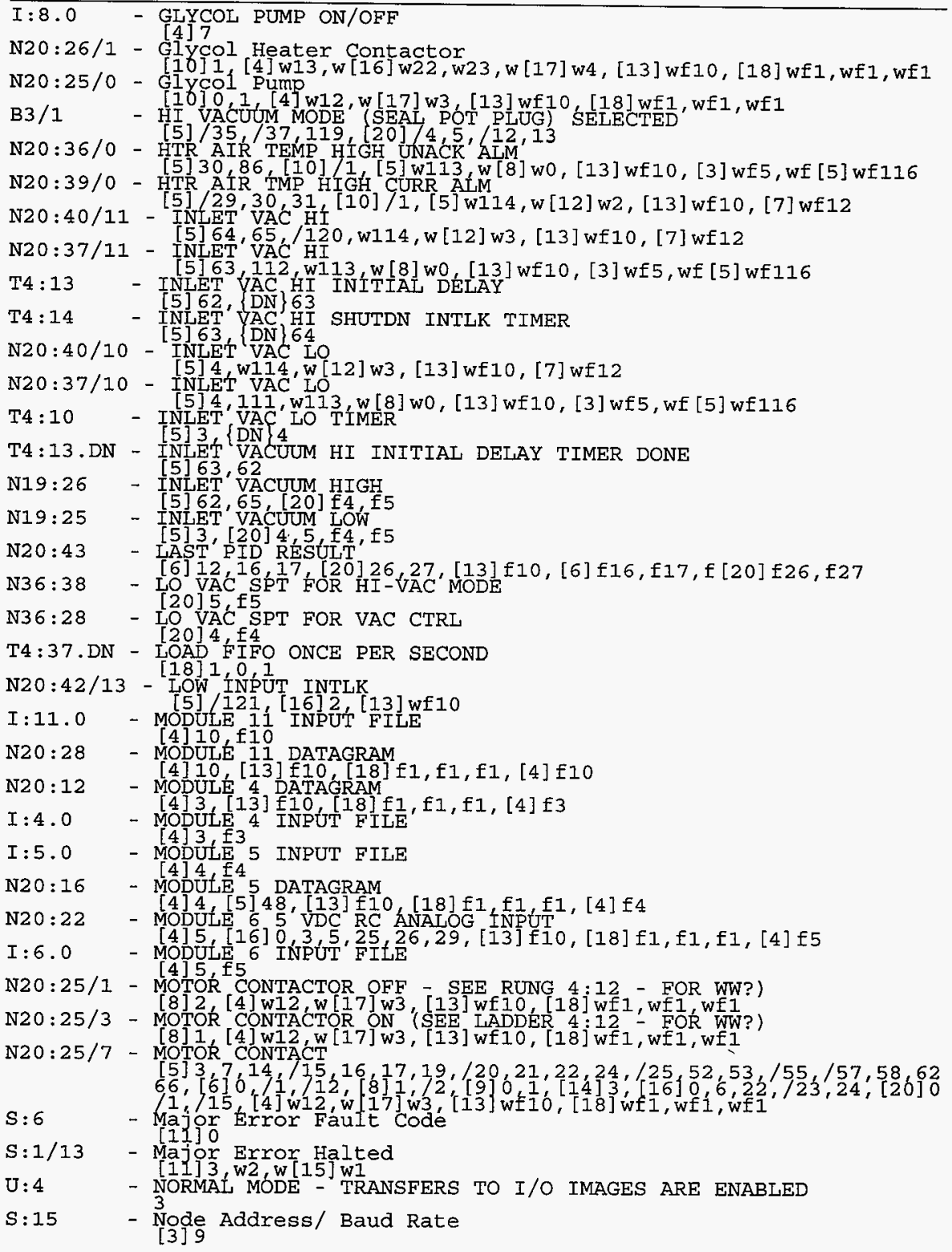




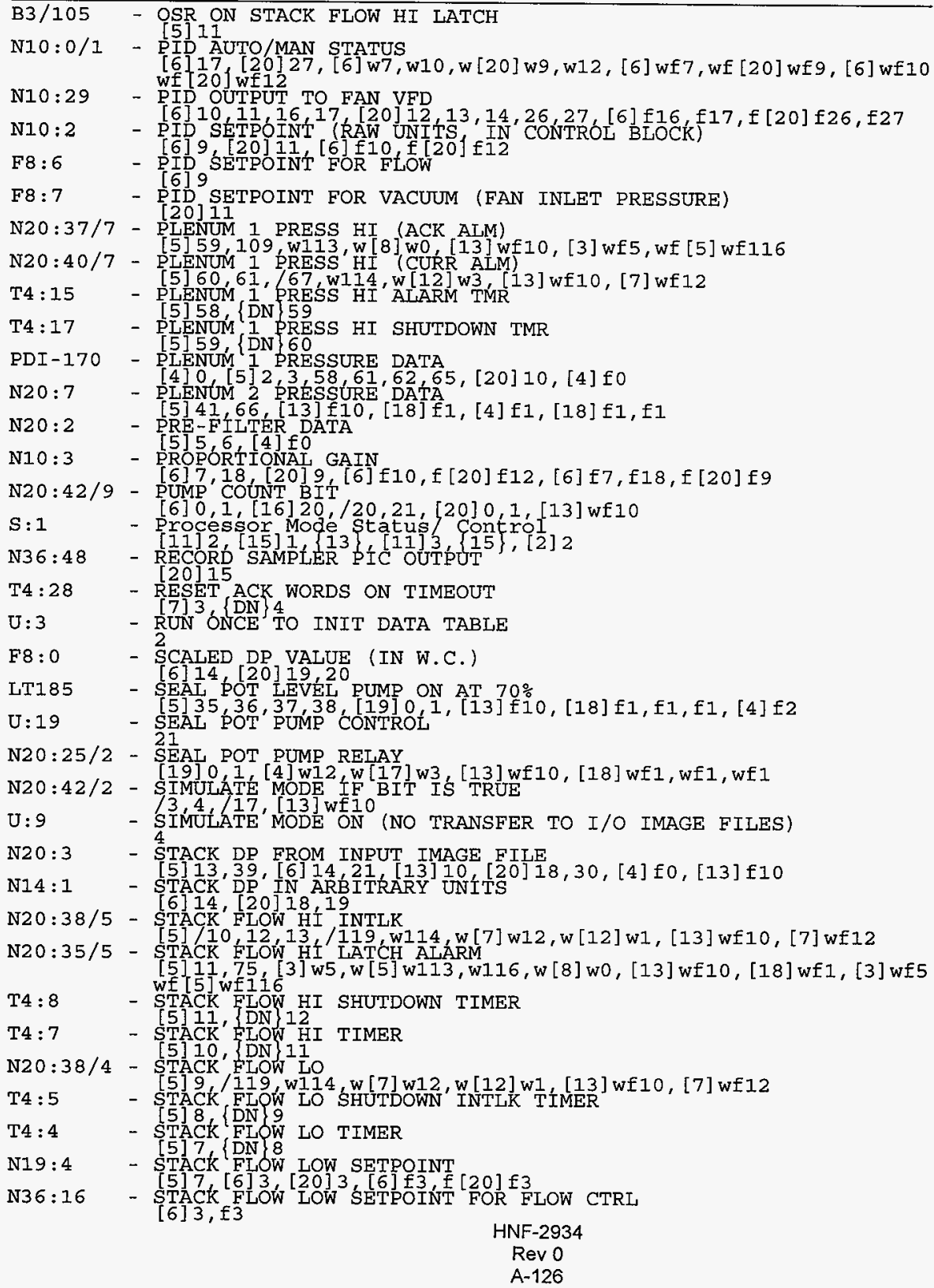




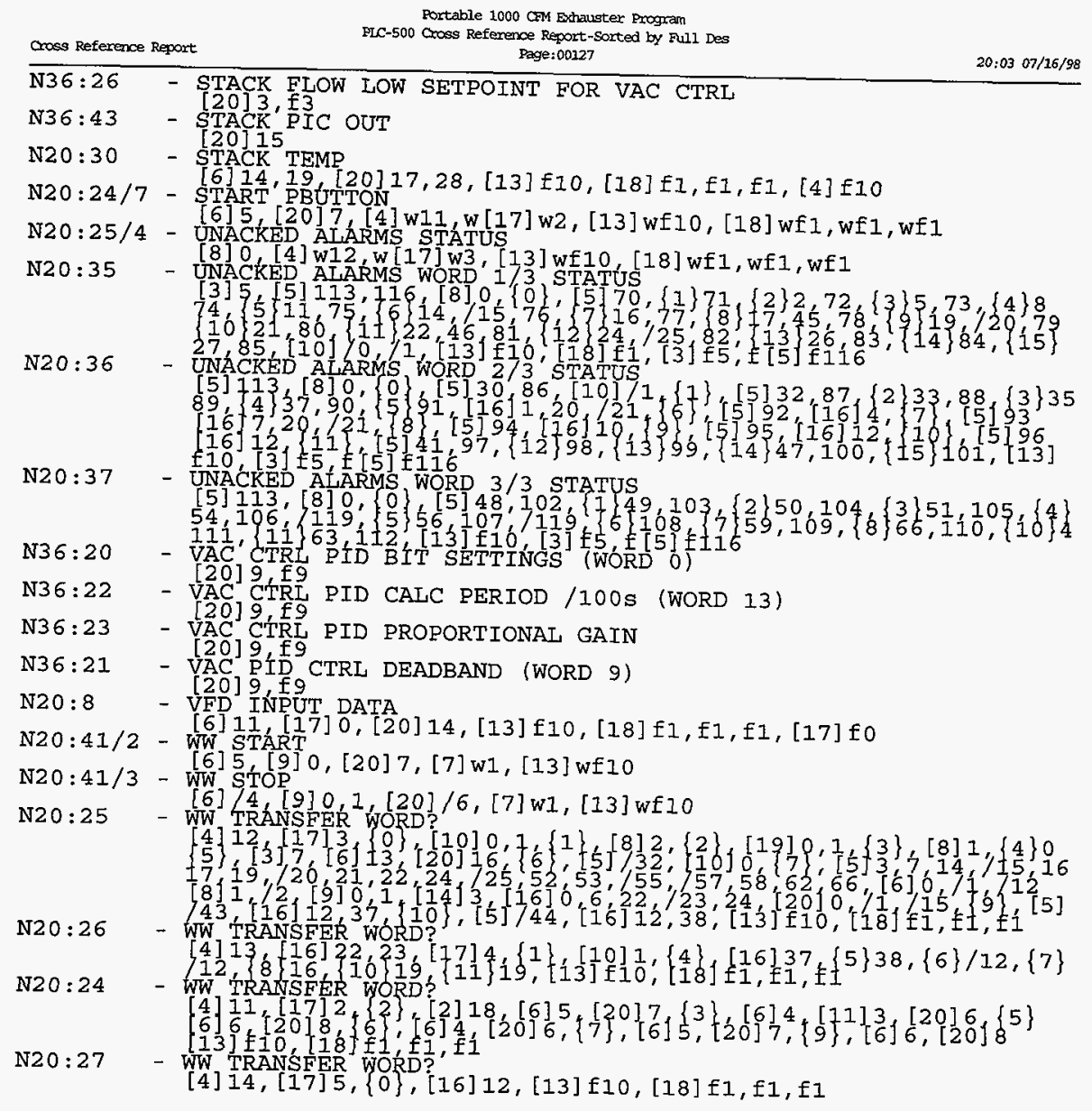


Data File \#0 Type: 0 output Elements:48 Nonds:48

\begin{tabular}{|c|c|c|c|c|c|c|c|}
\hline Address & 15 --- Data --.- 0 & Adidress & 15 -..- Data --- & Adtress & $15---$ Dat & ta $=$ & - \\
\hline $0: 3.0$ & $\operatorname{xxxx} x x \times x \operatorname{xxxx} x \times x x$ & $0: 12.0$ & $\mathrm{xxxx} x \mathrm{xxx} \times \mathrm{xxxx} \mathrm{xxxx}$ & $0: 12.16$ & 00000000 & 0000 & 0000 \\
\hline $0: 3.1$ & $\mathrm{xxxx} \operatorname{xxxx} \mathrm{xxxx} \mathrm{xxxx}$ & $0: 12.1$ & $x \times x x \operatorname{xxx} x \times x x \quad x x x x$ & $0: 12.17$ & 00000000 & 0000 & 0000 \\
\hline $0: 6.0$ & $\mathrm{xxxx} \operatorname{xxxx} \mathrm{xxxx} \mathrm{xxxx}$ & $0: 12.2$ & $x \times x \times x \times x \times x \times x \times x$ & $0: 12.18$ & 00000000 & 0000 & 0000 \\
\hline $0: 6.1$ & $x x x x \quad x x x x \quad x x x x \quad x x x x$ & $0: 12.3$ & 0000000000000000 & $0: 12.19$ & 00000000 & 0000 & 0000 \\
\hline $0: 7.0$ & $\mathrm{xx} \times x \times x$ & $0: 12,4$ & $\operatorname{xxxx} x x x x \operatorname{xxx} x x x x$ & $0: 12.20$ & 00000000 & 0000 & 0000 \\
\hline $0: 8.0$ & $x \times \cos x$ & $0: 12.5$ & 0000000000000000 & $0: 12.21$ & 00000000 & 0000 & 0000 \\
\hline $0: 9.0$ & $x x x x x$ & $0: 12.6$ & 0000000000000000 & $0: 12.22$ & 00000000 & 0000 & 0000 \\
\hline $0: 10.0$ & $x x x x x x$ & $0: 12.7$ & 0000000000000000 & $0: 12.23$ & 00000000 & 0000 & 0000 \\
\hline $0: 11.0$ & $\operatorname{cxx} x \operatorname{xxx} x \operatorname{cosx}$ & $0: 12.8$ & $\operatorname{xxcx} \cos x \operatorname{xxx} \operatorname{cosx}$ & $0: 12.24$ & 00000000 & 0000 & 0000 \\
\hline $0: 11.1$ & $\operatorname{cxx} \operatorname{xxx} x \operatorname{xxx}$ & $0: 12.9$ & $\operatorname{xxxx} x x x x \operatorname{xxx} x x x x$ & $0: 12.25$ & 00000000 & 0000 & 0000 \\
\hline $0: 11.2$ & $\operatorname{coxx} \cos x \cos x \operatorname{xox} x$ & $0: 12.10$ & $\operatorname{xxx} \operatorname{xxx} x x x x \operatorname{xxxx}$ & $0: 12.26$ & 00000000 & 0000 & 0000 \\
\hline $0: 11.3$ & $\cos x \operatorname{xxx} x \cos x \cos x$ & $0: 12.11$ & $\operatorname{xxxx} \operatorname{xxxx} x x x x \quad x x x x$ & $0: 12.27$ & 00000000 & 0000 & 0000 \\
\hline $0: 11.4$ & 0000000000000000 & $0: 12.12$ & 0000000000000000 & $0.12,28$ & 00000000 & 0000 & 0000 \\
\hline $0: 11.5$ & 0000000000000000 & $0: 12.13$ & 0000000000000000 & $0: 12.29$ & 00000000 & 0000 & 0000 \\
\hline $0: 31.6$ & 0000000000000000 & $0: 12.14$ & 0000000000000000 & $0: 12.30$ & 00000000 & 0000 & 0000 \\
\hline $0: 11.7$ & $00000000 \quad 0000 \quad 0000$ & $0: 12.15$ & 0000000000000000 & $0: 12.31$ & 00000000 & 0000 & 0000 \\
\hline
\end{tabular}

Data File \#1 Type:I Input Elements:64 Horis:64

Address 15 ---- Data --.- 0

I:1.0

I:1.1

I:1.2

I: 1.3

$I: 2.0$

$I: 2.1$

$I: 2.2$

I:2.3

$I: 3.0$

$I: 3.1$

$I: 4.0$

$I: 4.1$

$I: 4,2$

$I: 4.3$

$I: 5.0$

I:5.1

$I: 5.2$

$I: 5.3$

$I: 6.0$

$I: 6.1$

$I: 7,0$

$I: 8.0$

$\operatorname{xxxx} \operatorname{xoxx} x \operatorname{xxx} \operatorname{xxx}$

$\operatorname{xxxx} \operatorname{xxxx} x \times x x \operatorname{xxxx}$

$\mathrm{xxxx} \mathrm{xxxx} \mathrm{xxxx} \mathrm{xxxx}$

$\cos x \operatorname{six} x \operatorname{sos} x \operatorname{sic} x$

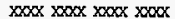

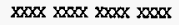

$\operatorname{xxxx} x x x x \operatorname{xxx} x x x x$

$\mathrm{xxxx} x \mathrm{xxx} \times \mathrm{xxxx} \mathrm{xxxx}$

$\operatorname{xxxx} \operatorname{xxxx} \mathrm{xxxx} \times \mathrm{xxxx}$

$\mathrm{xxxx} \mathrm{xxxx} \mathrm{xxxx} \mathrm{xxxx}$

$\mathrm{xxxx} \mathrm{xxxx} \mathrm{xxxx} \mathrm{xxxx}$

$\mathrm{xxx} \operatorname{sxxx} \mathrm{xox} x \mathrm{xox}$

$\operatorname{xoxx} \operatorname{xox} x \operatorname{xxx} x \operatorname{xxx}$

$\operatorname{xxxx} x x x x \operatorname{xxxx} x x x x$

$\mathrm{xxxx} \mathrm{xxxx} \mathrm{xxxx} \mathrm{xxxx}$

$\mathrm{xxxx} \mathrm{xxxx} \mathrm{xxxx} \mathrm{xxxx}$

$\mathrm{xxxx} \mathrm{xxxx} \mathrm{xxxx} \mathrm{xxxx}$

$\mathrm{xxxx} \operatorname{xox} x \mathrm{xox} x \mathrm{xxx}$

$\mathrm{xox} x \operatorname{xox} x \operatorname{xox} x \operatorname{xox}$

$\mathrm{xxox} \operatorname{xxxx} \mathrm{xxxx} x \mathrm{xxx}$

$\mathrm{xx} \mathrm{xxxx}$

$\mathrm{xx} \mathrm{xxxx}$

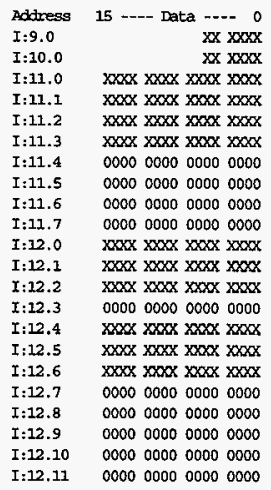

Data File \#2 Type:S Status Elements:83 Woxds:83

Adaregs -....-$\$ 2: 0$ $52: 50$
1111111111222222222233333333334444444444 01234567890123456789012345678901234567890123456789 $\mathbf{x} x \mathbf{x}$

$$
\mathrm{X}
$$

Data File \#3 Type:B Binary Elements:35 Words:35

\begin{tabular}{|c|c|c|c|c|c|}
\hline Address & $15 \ldots$ Data -... & Address & $15---D a$ & ta -- & - \\
\hline $\mathrm{B} 3 / 00000$ & $0000000000000 \mathrm{xxx}$ & $\mathrm{B} 3 / 00192$ & $x 000 \times 000$ & 0000 & 0000 \\
\hline $3 / 00016$ & $000 x \times x \times x \times 00000 x x$ & $\mathrm{~B} 3 / 00208$ & 00000000 & $x 000$ & $0 \times 00$ \\
\hline $\mathrm{B} 3 / 00032$ & $x x x x \quad x x x x \quad x x x x \quad x x x x$ & $\mathrm{~B} 3 / 00224$ & 00000000 & $\mathrm{xx} \times 0$ & 0000 \\
\hline $\mathrm{B} 3 / 00048$ & $00000000 \times \times 00$ & $\mathrm{~B} 3 / 00240$ & 00000000 & 0000 & 0000 \\
\hline $\mathrm{B} 3 / 00064$ & $00 \times x \times x \times x 00000000$ & B3/00256 & 00000000 & 0000 & 0000 \\
\hline $\mathrm{B} 3 / 00080$ & 0000000000000000 & $\mathrm{~B} 3 / 00272$ & 00000000 & 0000 & 0000 \\
\hline B3/00096 & $00 \times 0 \quad 0 \times x \times 0 \times 0000000$ & $\mathrm{~B} 3 / 00288$ & 00000000 & 0000 & 0000 \\
\hline $\mathrm{B} 3 / 00112$ & $0 \times 000000 \times 000 x \times x 0 x$ & $\mathrm{~B} 3 / 00304$ & 00000000 & 0000 & 0000 \\
\hline $\mathrm{B} 3 / 00128$ & $x \times x 0000000 \times x \times x \times 0 x$ & $\mathrm{~B} 3 / 00320$ & 00000000 & 0000 & 0000 \\
\hline $\mathrm{B} 3 / 00144$ & $00 x 0 \operatorname{cxxx} x \operatorname{cox} 0 \operatorname{xxx}$ & B3/00336 & 00000000 & 0000 & 0000 \\
\hline $\mathrm{B} 3 / 00160$ & $00000000000000 \mathrm{xx}$ & B3/00352 & 00000000 & 0000 & 0000 \\
\hline $\mathrm{B} 3 / 00176$ & 0000000000000000 & $\mathrm{~B} 3 / 00368$ & 00000000 & 0000 & 0000 \\
\hline
\end{tabular}

$\begin{array}{ll}\text { Address } & 15-\cdots \text { Data }-\cdots \\ I: 12.12 & 0000000000000000 \\ I: 12.13 & 0000000000000000 \\ I: 12.14 & 0000000000000000 \\ I: 12.15 & 0000000000000000 \\ I: 12.16 & 000000000000000 \\ I: 12.17 & 0000000000000000 \\ I: 12.18 & 0000000000000000 \\ I: 12.19 & 0000000000000000 \\ I: 12.20 & 0000000000000000 \\ I: 12.21 & 0000000000000000 \\ I: 12.22 & 0000000000000000 \\ I: 12.23 & 0000000000000000 \\ I: 12.24 & 0000000000000000 \\ I: 12.25 & 0000000000000000 \\ I: 12.26 & 0000000000000000 \\ I: 12.27 & 0000000000000000 \\ I: 12.28 & 0000000000000000 \\ I: 12.29 & 0000000000000000 \\ I: 12.30 & 0000000000000000 \\ I: 12.31 & 0000000000000000 \\ & \\ & \end{array}$

$\begin{array}{ll}\text { Address } & 15-1 \\ I: 12.12 & 0000000000000000 \\ I: 12.13 & 0000000000000000 \\ I: 12.14 & 0000000000000000 \\ I: 12.15 & 0000000000000000 \\ I: 12.16 & 0000000000000000 \\ I: 12.17 & 0000000000000000 \\ I: 12.18 & 0000000000000000 \\ I: 12.19 & 0000000000000000 \\ I: 12.20 & 0000000000000000 \\ I: 12.21 & 0000000000000000 \\ I: 12.22 & 0000000000000000 \\ I: 12.23 & 0000000000000000 \\ I: 12.24 & 0000000000000000 \\ I: 12.25 & 0000000000000000 \\ I: 12.26 & 0000000000000000 \\ I: 12.27 & 0000000000000000 \\ I: 12.28 & 0000000000000000 \\ I: 12.29 & 0000000000000000 \\ I: 12.30 & 0000000000000000 \\ I: 12.31 & 0000000000000000 \\ & \\ & \end{array}$

$\begin{array}{ll}\text { Adtress } & 15-1 \text { Data }-\cdots \\ I: 12.12 & 0000000000000000 \\ I: 12.13 & 0000000000000000 \\ I: 12.14 & 0000000000000000 \\ I: 12.15 & 0000000000000000 \\ I: 12.16 & 0000000000000000 \\ I: 12.17 & 0000000000000000 \\ I: 12.18 & 0000000000000000 \\ I: 12.19 & 0000000000000000 \\ I: 12.20 & 0000000000000000 \\ I: 12.21 & 0000000000000000 \\ I: 12.22 & 0000000000000000 \\ I: 12.23 & 0000000000000000 \\ I: 12.24 & 0000000000000000 \\ I: 12.25 & 0000000000000000 \\ I: 12.26 & 0000000000000000 \\ I: 12.27 & 0000000000000000 \\ I: 12.28 & 0000000000000000 \\ I: 12.29 & 0000000000000000 \\ I: 12.30 & 0000000000000000 \\ I: 12.31 & 0000000000000000 \\ & \\ & \end{array}$

$\begin{array}{ll}\text { Address } & 15-\cdots \text { Data }-\cdots \\ I: 12.12 & 0000000000000000 \\ I: 12.13 & 0000000000000000 \\ I: 12.14 & 0000000000000000 \\ I: 12.15 & 0000000000000000 \\ I: 12.16 & 000000000000000 \\ I: 12.17 & 0000000000000000 \\ I: 12.18 & 0000000000000000 \\ I: 12.19 & 0000000000000000 \\ I: 12.20 & 0000000000000000 \\ I: 12.21 & 0000000000000000 \\ I: 12.22 & 0000000000000000 \\ I: 12.23 & 0000000000000000 \\ I: 12.24 & 0000000000000000 \\ I: 12.25 & 0000000000000000 \\ I: 12.26 & 0000000000000000 \\ I: 12.27 & 0000000000000000 \\ I: 12.28 & 0000000000000000 \\ I: 12.29 & 0000000000000000 \\ I: 12.30 & 0000000000000000 \\ I: 12.31 & 0000000000000000 \\ & \\ & \end{array}$

$\begin{array}{ll}\text { Address } & 15-\cdots \text { Data }-\cdots \\ I: 12.12 & 0000000000000000 \\ I: 12.13 & 0000000000000000 \\ I: 12.14 & 0000000000000000 \\ I: 12.15 & 0000000000000000 \\ I: 12.16 & 000000000000000 \\ I: 12.17 & 0000000000000000 \\ I: 12.18 & 0000000000000000 \\ I: 12.19 & 0000000000000000 \\ I: 12.20 & 0000000000000000 \\ I: 12.21 & 0000000000000000 \\ I: 12.22 & 0000000000000000 \\ I: 12.23 & 0000000000000000 \\ I: 12.24 & 0000000000000000 \\ I: 12.25 & 0000000000000000 \\ I: 12.26 & 0000000000000000 \\ I: 12.27 & 0000000000000000 \\ I: 12.28 & 0000000000000000 \\ I: 12.29 & 0000000000000000 \\ I: 12.30 & 0000000000000000 \\ I: 12.31 & 0000000000000000 \\ & \\ & \end{array}$

$\begin{array}{ll}\text { Adtress } & 15-1 \text { Data }-\cdots \\ I: 12.12 & 0000000000000000 \\ I: 12.13 & 0000000000000000 \\ I: 12.14 & 0000000000000000 \\ I: 12.15 & 0000000000000000 \\ I: 12.16 & 0000000000000000 \\ I: 12.17 & 0000000000000000 \\ I: 12.18 & 0000000000000000 \\ I: 12.19 & 0000000000000000 \\ I: 12.20 & 0000000000000000 \\ I: 12.21 & 0000000000000000 \\ I: 12.22 & 0000000000000000 \\ I: 12.23 & 0000000000000000 \\ I: 12.24 & 0000000000000000 \\ I: 12.25 & 0000000000000000 \\ I: 12.26 & 0000000000000000 \\ I: 12.27 & 0000000000000000 \\ I: 12.28 & 0000000000000000 \\ I: 12.29 & 0000000000000000 \\ I: 12.30 & 0000000000000000 \\ I: 12.31 & 0000000000000000 \\ & \\ & \end{array}$

$\begin{array}{ll}\text { Address } & 15-\cdots \text { Data }-\cdots \\ I: 12.12 & 0000000000000000 \\ I: 12.13 & 0000000000000000 \\ I: 12.14 & 0000000000000000 \\ I: 12.15 & 0000000000000000 \\ I: 12.16 & 000000000000000 \\ I: 12.17 & 0000000000000000 \\ I: 12.18 & 0000000000000000 \\ I: 12.19 & 0000000000000000 \\ I: 12.20 & 0000000000000000 \\ I: 12.21 & 0000000000000000 \\ I: 12.22 & 0000000000000000 \\ I: 12.23 & 0000000000000000 \\ I: 12.24 & 0000000000000000 \\ I: 12.25 & 0000000000000000 \\ I: 12.26 & 0000000000000000 \\ I: 12.27 & 0000000000000000 \\ I: 12.28 & 0000000000000000 \\ I: 12.29 & 0000000000000000 \\ I: 12.30 & 0000000000000000 \\ I: 12.31 & 0000000000000000 \\ & \\ & \end{array}$

$\begin{array}{ll}\text { Address } & 15-1 \\ I: 12.12 & 0000000000000000 \\ I: 12.13 & 0000000000000000 \\ I: 12.14 & 0000000000000000 \\ I: 12.15 & 0000000000000000 \\ I: 12.16 & 0000000000000000 \\ I: 12.17 & 0000000000000000 \\ I: 12.18 & 0000000000000000 \\ I: 12.19 & 0000000000000000 \\ I: 12.20 & 0000000000000000 \\ I: 12.21 & 0000000000000000 \\ I: 12.22 & 0000000000000000 \\ I: 12.23 & 0000000000000000 \\ I: 12.24 & 0000000000000000 \\ I: 12.25 & 0000000000000000 \\ I: 12.26 & 0000000000000000 \\ I: 12.27 & 0000000000000000 \\ I: 12.28 & 0000000000000000 \\ I: 12.29 & 0000000000000000 \\ I: 12.30 & 0000000000000000 \\ I: 12.31 & 0000000000000000 \\ & \\ & \end{array}$

$\begin{array}{ll}\text { Address } & 15-\cdots \text { Data }-\cdots \\ I: 12.12 & 0000000000000000 \\ I: 12.13 & 0000000000000000 \\ I: 12.14 & 0000000000000000 \\ I: 12.15 & 0000000000000000 \\ I: 12.16 & 000000000000000 \\ I: 12.17 & 0000000000000000 \\ I: 12.18 & 0000000000000000 \\ I: 12.19 & 0000000000000000 \\ I: 12.20 & 0000000000000000 \\ I: 12.21 & 0000000000000000 \\ I: 12.22 & 0000000000000000 \\ I: 12.23 & 0000000000000000 \\ I: 12.24 & 0000000000000000 \\ I: 12.25 & 0000000000000000 \\ I: 12.26 & 0000000000000000 \\ I: 12.27 & 0000000000000000 \\ I: 12.28 & 0000000000000000 \\ I: 12.29 & 0000000000000000 \\ I: 12.30 & 0000000000000000 \\ I: 12.31 & 0000000000000000 \\ & \\ & \end{array}$

$\begin{array}{ll}\text { Address } & 15-1 \\ I: 12.12 & 0000000000000000 \\ I: 12.13 & 0000000000000000 \\ I: 12.14 & 0000000000000000 \\ I: 12.15 & 0000000000000000 \\ I: 12.16 & 0000000000000000 \\ I: 12.17 & 0000000000000000 \\ I: 12.18 & 0000000000000000 \\ I: 12.19 & 0000000000000000 \\ I: 12.20 & 0000000000000000 \\ I: 12.21 & 0000000000000000 \\ I: 12.22 & 0000000000000000 \\ I: 12.23 & 0000000000000000 \\ I: 12.24 & 0000000000000000 \\ I: 12.25 & 0000000000000000 \\ I: 12.26 & 0000000000000000 \\ I: 12.27 & 0000000000000000 \\ I: 12.28 & 0000000000000000 \\ I: 12.29 & 0000000000000000 \\ I: 12.30 & 0000000000000000 \\ I: 12.31 & 0000000000000000 \\ & \\ & \end{array}$

$\begin{array}{ll}\text { Address } & 15-1 \\ I: 12.12 & 0000000000000000 \\ I: 12.13 & 0000000000000000 \\ I: 12.14 & 0000000000000000 \\ I: 12.15 & 0000000000000000 \\ I: 12.16 & 0000000000000000 \\ I: 12.17 & 0000000000000000 \\ I: 12.18 & 0000000000000000 \\ I: 12.19 & 0000000000000000 \\ I: 12.20 & 0000000000000000 \\ I: 12.21 & 0000000000000000 \\ I: 12.22 & 0000000000000000 \\ I: 12.23 & 0000000000000000 \\ I: 12.24 & 0000000000000000 \\ I: 12.25 & 0000000000000000 \\ I: 12.26 & 0000000000000000 \\ I: 12.27 & 0000000000000000 \\ I: 12.28 & 0000000000000000 \\ I: 12.29 & 0000000000000000 \\ I: 12.30 & 0000000000000000 \\ I: 12.31 & 0000000000000000 \\ & \\ & \end{array}$

$\begin{array}{ll}\text { Address } & 15-1 \\ I: 12.12 & 0000000000000000 \\ I: 12.13 & 0000000000000000 \\ I: 12.14 & 0000000000000000 \\ I: 12.15 & 0000000000000000 \\ I: 12.16 & 0000000000000000 \\ I: 12.17 & 0000000000000000 \\ I: 12.18 & 0000000000000000 \\ I: 12.19 & 0000000000000000 \\ I: 12.20 & 0000000000000000 \\ I: 12.21 & 0000000000000000 \\ I: 12.22 & 0000000000000000 \\ I: 12.23 & 0000000000000000 \\ I: 12.24 & 0000000000000000 \\ I: 12.25 & 0000000000000000 \\ I: 12.26 & 0000000000000000 \\ I: 12.27 & 0000000000000000 \\ I: 12.28 & 0000000000000000 \\ I: 12.29 & 0000000000000000 \\ I: 12.30 & 0000000000000000 \\ I: 12.31 & 0000000000000000 \\ & \\ & \end{array}$

$\begin{array}{ll}\text { Address } & 15-\cdots \text { Data }-\cdots \\ I: 12.12 & 0000000000000000 \\ I: 12.13 & 0000000000000000 \\ I: 12.14 & 0000000000000000 \\ I: 12.15 & 0000000000000000 \\ I: 12.16 & 000000000000000 \\ I: 12.17 & 0000000000000000 \\ I: 12.18 & 0000000000000000 \\ I: 12.19 & 0000000000000000 \\ I: 12.20 & 0000000000000000 \\ I: 12.21 & 0000000000000000 \\ I: 12.22 & 0000000000000000 \\ I: 12.23 & 0000000000000000 \\ I: 12.24 & 0000000000000000 \\ I: 12.25 & 0000000000000000 \\ I: 12.26 & 0000000000000000 \\ I: 12.27 & 0000000000000000 \\ I: 12.28 & 0000000000000000 \\ I: 12.29 & 0000000000000000 \\ I: 12.30 & 0000000000000000 \\ I: 12.31 & 0000000000000000 \\ & \\ & \end{array}$

$\begin{array}{ll}\text { Address } & 15-1 \\ I: 12.12 & 0000000000000000 \\ I: 12.13 & 0000000000000000 \\ I: 12.14 & 0000000000000000 \\ I: 12.15 & 0000000000000000 \\ I: 12.16 & 0000000000000000 \\ I: 12.17 & 0000000000000000 \\ I: 12.18 & 0000000000000000 \\ I: 12.19 & 0000000000000000 \\ I: 12.20 & 0000000000000000 \\ I: 12.21 & 0000000000000000 \\ I: 12.22 & 0000000000000000 \\ I: 12.23 & 0000000000000000 \\ I: 12.24 & 0000000000000000 \\ I: 12.25 & 0000000000000000 \\ I: 12.26 & 0000000000000000 \\ I: 12.27 & 0000000000000000 \\ I: 12.28 & 0000000000000000 \\ I: 12.29 & 0000000000000000 \\ I: 12.30 & 0000000000000000 \\ I: 12.31 & 0000000000000000 \\ & \\ & \end{array}$

$\begin{array}{ll}\text { Adtress } & 15-1 \text { Data }-\cdots \\ I: 12.12 & 0000000000000000 \\ I: 12.13 & 0000000000000000 \\ I: 12.14 & 0000000000000000 \\ I: 12.15 & 0000000000000000 \\ I: 12.16 & 0000000000000000 \\ I: 12.17 & 0000000000000000 \\ I: 12.18 & 0000000000000000 \\ I: 12.19 & 0000000000000000 \\ I: 12.20 & 0000000000000000 \\ I: 12.21 & 0000000000000000 \\ I: 12.22 & 0000000000000000 \\ I: 12.23 & 0000000000000000 \\ I: 12.24 & 0000000000000000 \\ I: 12.25 & 0000000000000000 \\ I: 12.26 & 0000000000000000 \\ I: 12.27 & 0000000000000000 \\ I: 12.28 & 0000000000000000 \\ I: 12.29 & 0000000000000000 \\ I: 12.30 & 0000000000000000 \\ I: 12.31 & 0000000000000000 \\ & \\ & \end{array}$

$\begin{array}{ll}\text { Address } & 15-1 \\ I: 12.12 & 0000000000000000 \\ I: 12.13 & 0000000000000000 \\ I: 12.14 & 0000000000000000 \\ I: 12.15 & 0000000000000000 \\ I: 12.16 & 0000000000000000 \\ I: 12.17 & 0000000000000000 \\ I: 12.18 & 0000000000000000 \\ I: 12.19 & 0000000000000000 \\ I: 12.20 & 0000000000000000 \\ I: 12.21 & 0000000000000000 \\ I: 12.22 & 0000000000000000 \\ I: 12.23 & 0000000000000000 \\ I: 12.24 & 0000000000000000 \\ I: 12.25 & 0000000000000000 \\ I: 12.26 & 0000000000000000 \\ I: 12.27 & 0000000000000000 \\ I: 12.28 & 0000000000000000 \\ I: 12.29 & 0000000000000000 \\ I: 12.30 & 0000000000000000 \\ I: 12.31 & 0000000000000000 \\ & \\ & \end{array}$

$\begin{array}{ll}\text { Adtress } & 15-1 \text { Data }-\cdots \\ I: 12.12 & 0000000000000000 \\ I: 12.13 & 0000000000000000 \\ I: 12.14 & 0000000000000000 \\ I: 12.15 & 0000000000000000 \\ I: 12.16 & 0000000000000000 \\ I: 12.17 & 0000000000000000 \\ I: 12.18 & 0000000000000000 \\ I: 12.19 & 0000000000000000 \\ I: 12.20 & 0000000000000000 \\ I: 12.21 & 0000000000000000 \\ I: 12.22 & 0000000000000000 \\ I: 12.23 & 0000000000000000 \\ I: 12.24 & 0000000000000000 \\ I: 12.25 & 0000000000000000 \\ I: 12.26 & 0000000000000000 \\ I: 12.27 & 0000000000000000 \\ I: 12.28 & 0000000000000000 \\ I: 12.29 & 0000000000000000 \\ I: 12.30 & 0000000000000000 \\ I: 12.31 & 0000000000000000 \\ & \\ & \end{array}$

$\begin{array}{ll}\text { Address } & 15-1 \\ I: 12.12 & 0000000000000000 \\ I: 12.13 & 0000000000000000 \\ I: 12.14 & 0000000000000000 \\ I: 12.15 & 0000000000000000 \\ I: 12.16 & 0000000000000000 \\ I: 12.17 & 0000000000000000 \\ I: 12.18 & 0000000000000000 \\ I: 12.19 & 0000000000000000 \\ I: 12.20 & 0000000000000000 \\ I: 12.21 & 0000000000000000 \\ I: 12.22 & 0000000000000000 \\ I: 12.23 & 0000000000000000 \\ I: 12.24 & 0000000000000000 \\ I: 12.25 & 0000000000000000 \\ I: 12.26 & 0000000000000000 \\ I: 12.27 & 0000000000000000 \\ I: 12.28 & 0000000000000000 \\ I: 12.29 & 0000000000000000 \\ I: 12.30 & 0000000000000000 \\ I: 12.31 & 0000000000000000 \\ & \\ & \end{array}$

$\begin{array}{ll}\text { Adtress } & 15-1 \text { Data }-\cdots \\ I: 12.12 & 0000000000000000 \\ I: 12.13 & 0000000000000000 \\ I: 12.14 & 0000000000000000 \\ I: 12.15 & 0000000000000000 \\ I: 12.16 & 0000000000000000 \\ I: 12.17 & 0000000000000000 \\ I: 12.18 & 0000000000000000 \\ I: 12.19 & 0000000000000000 \\ I: 12.20 & 0000000000000000 \\ I: 12.21 & 0000000000000000 \\ I: 12.22 & 0000000000000000 \\ I: 12.23 & 0000000000000000 \\ I: 12.24 & 0000000000000000 \\ I: 12.25 & 0000000000000000 \\ I: 12.26 & 0000000000000000 \\ I: 12.27 & 0000000000000000 \\ I: 12.28 & 0000000000000000 \\ I: 12.29 & 0000000000000000 \\ I: 12.30 & 0000000000000000 \\ I: 12.31 & 0000000000000000 \\ & \\ & \end{array}$

$\begin{array}{ll}\text { Address } & 15-1 \\ I: 12.12 & 0000000000000000 \\ I: 12.13 & 0000000000000000 \\ I: 12.14 & 0000000000000000 \\ I: 12.15 & 0000000000000000 \\ I: 12.16 & 0000000000000000 \\ I: 12.17 & 0000000000000000 \\ I: 12.18 & 0000000000000000 \\ I: 12.19 & 0000000000000000 \\ I: 12.20 & 0000000000000000 \\ I: 12.21 & 0000000000000000 \\ I: 12.22 & 0000000000000000 \\ I: 12.23 & 0000000000000000 \\ I: 12.24 & 0000000000000000 \\ I: 12.25 & 0000000000000000 \\ I: 12.26 & 0000000000000000 \\ I: 12.27 & 0000000000000000 \\ I: 12.28 & 0000000000000000 \\ I: 12.29 & 0000000000000000 \\ I: 12.30 & 0000000000000000 \\ I: 12.31 & 0000000000000000 \\ & \\ & \end{array}$

$\begin{array}{ll}\text { Address } & 15-1 \\ I: 12.12 & 0000000000000000 \\ I: 12.13 & 0000000000000000 \\ I: 12.14 & 0000000000000000 \\ I: 12.15 & 0000000000000000 \\ I: 12.16 & 0000000000000000 \\ I: 12.17 & 0000000000000000 \\ I: 12.18 & 0000000000000000 \\ I: 12.19 & 0000000000000000 \\ I: 12.20 & 0000000000000000 \\ I: 12.21 & 0000000000000000 \\ I: 12.22 & 0000000000000000 \\ I: 12.23 & 0000000000000000 \\ I: 12.24 & 0000000000000000 \\ I: 12.25 & 0000000000000000 \\ I: 12.26 & 0000000000000000 \\ I: 12.27 & 0000000000000000 \\ I: 12.28 & 0000000000000000 \\ I: 12.29 & 0000000000000000 \\ I: 12.30 & 0000000000000000 \\ I: 12.31 & 0000000000000000 \\ & \\ & \end{array}$

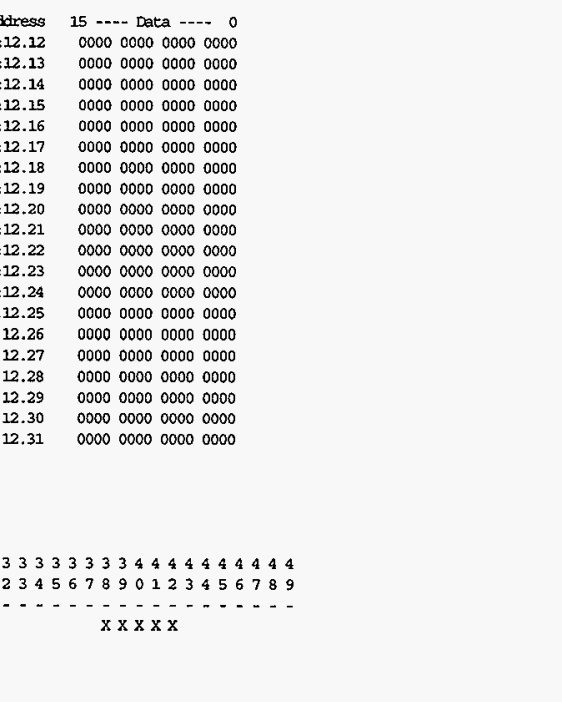

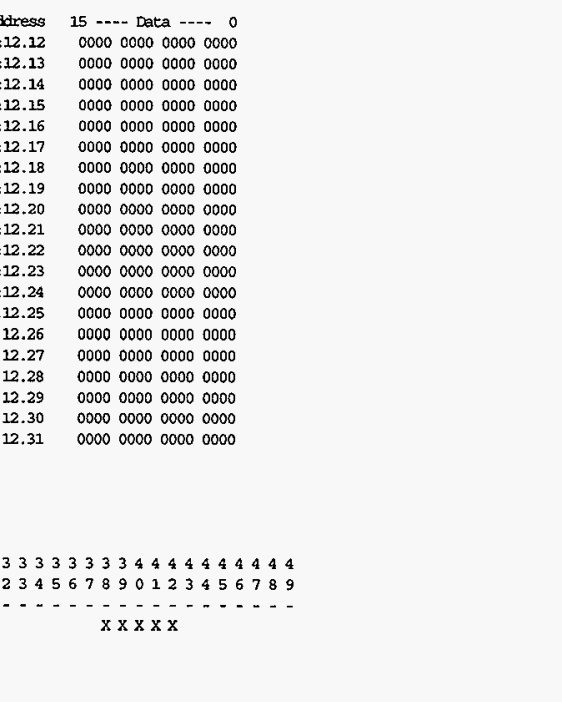

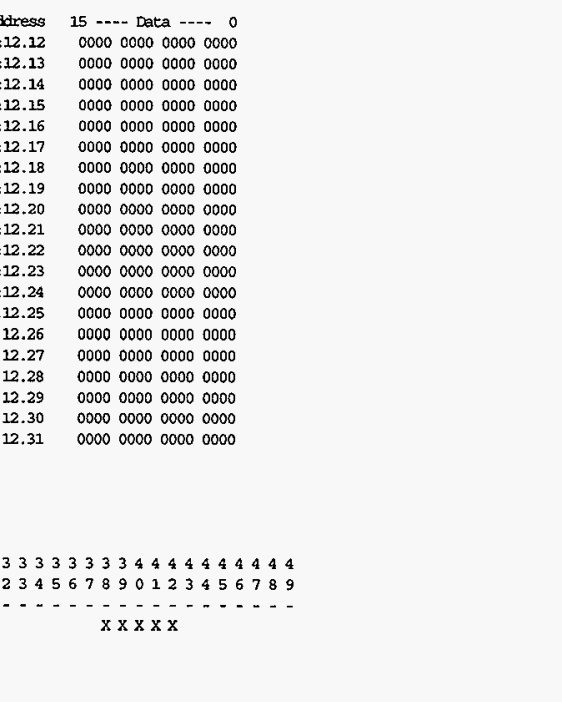

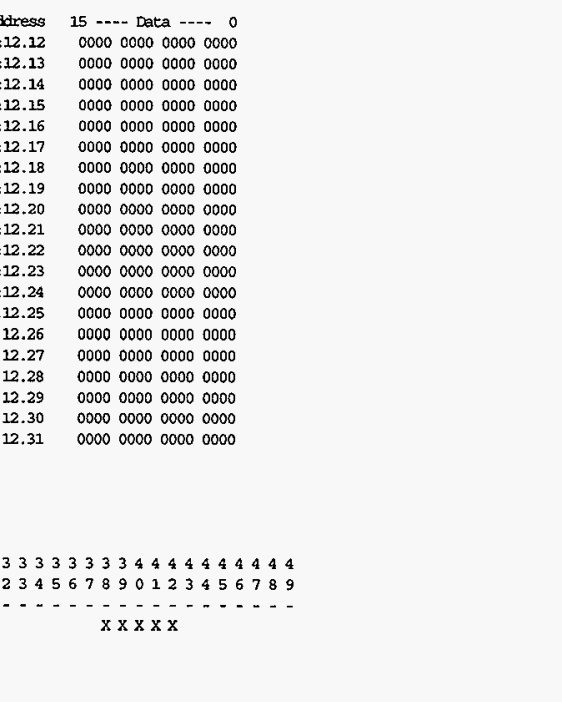

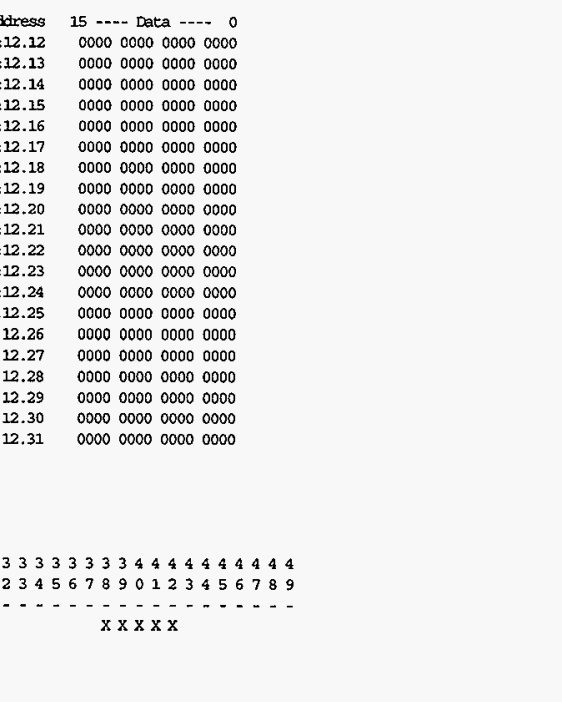

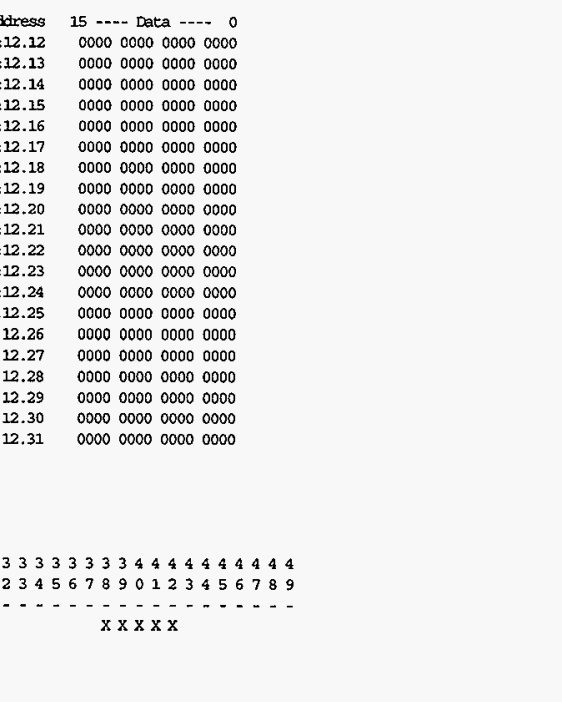

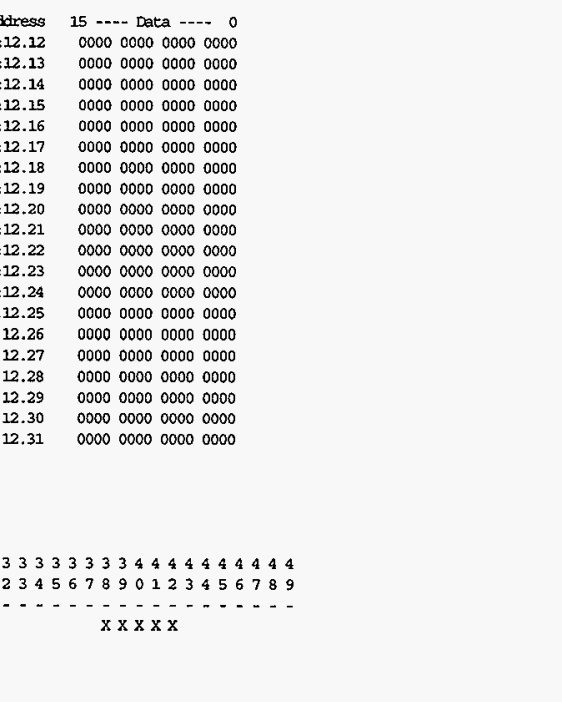

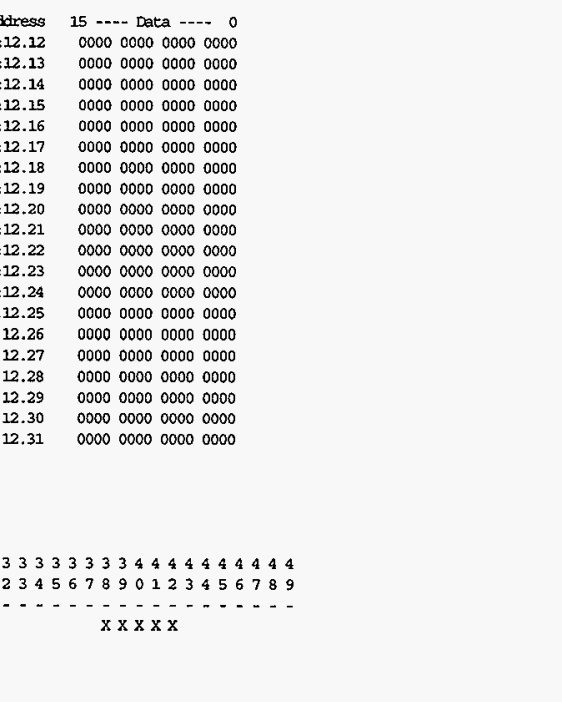

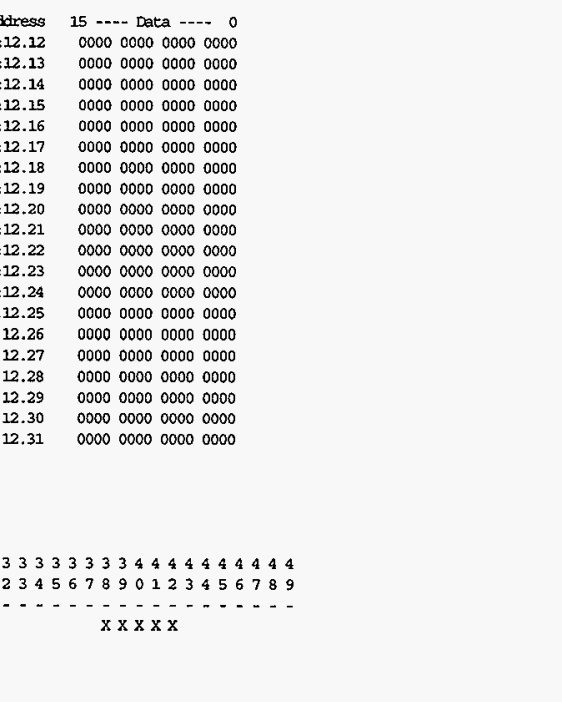

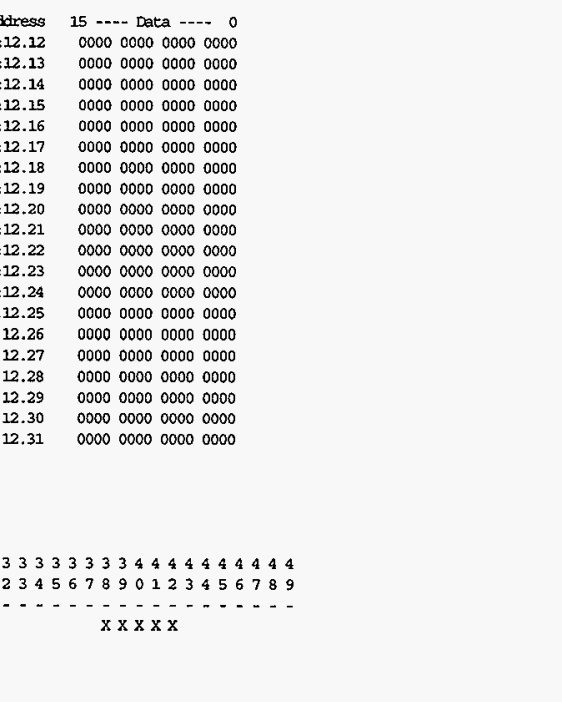

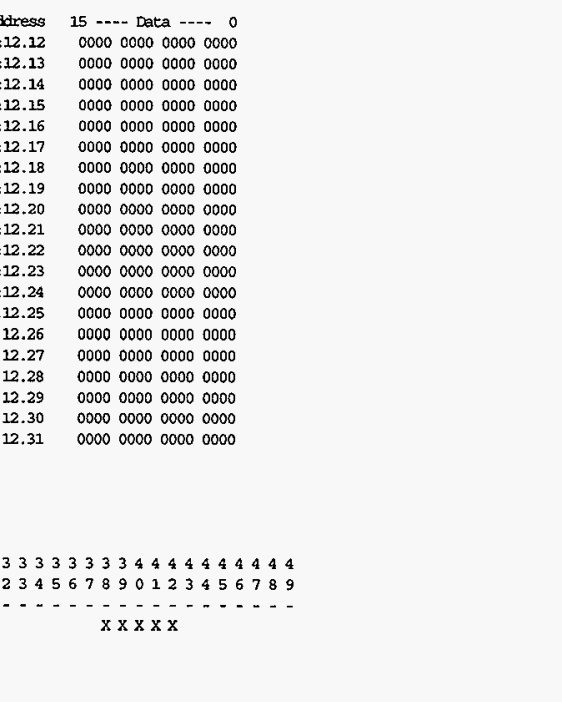

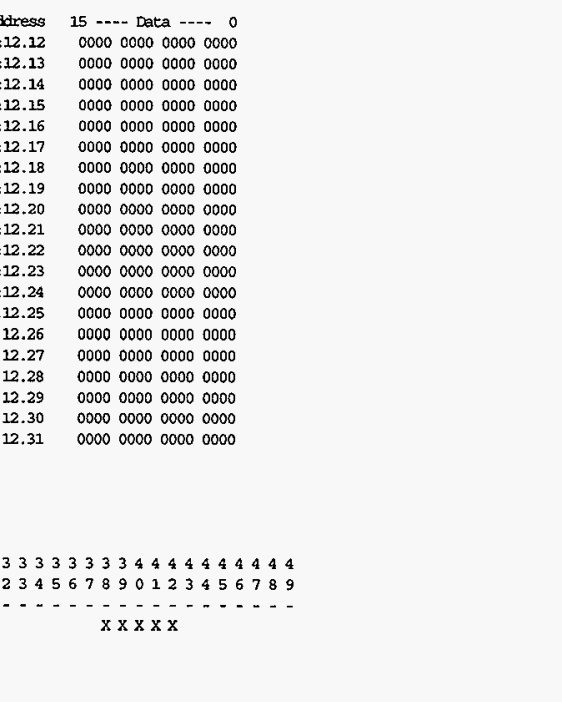

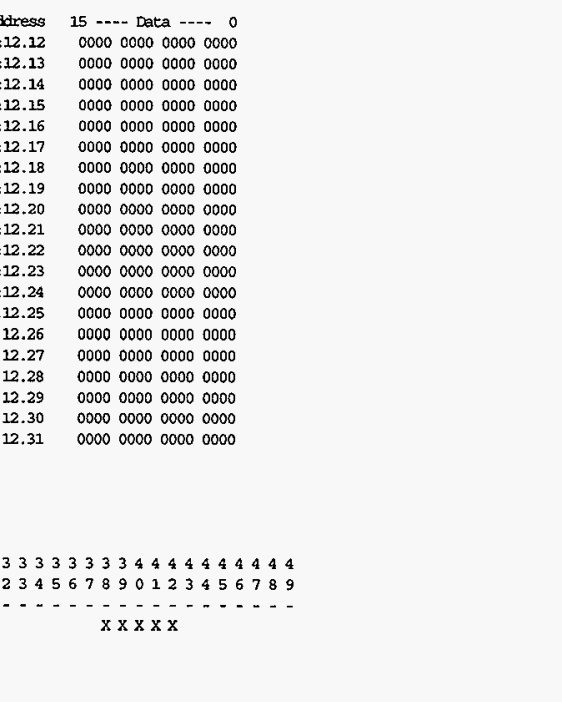

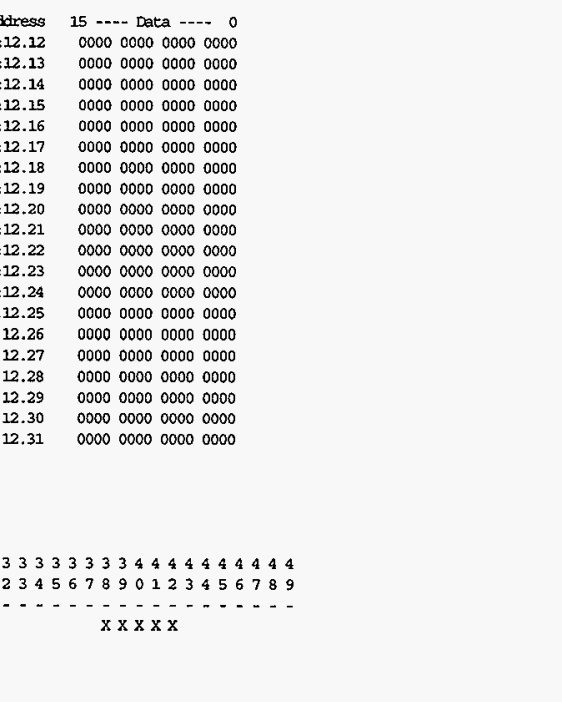

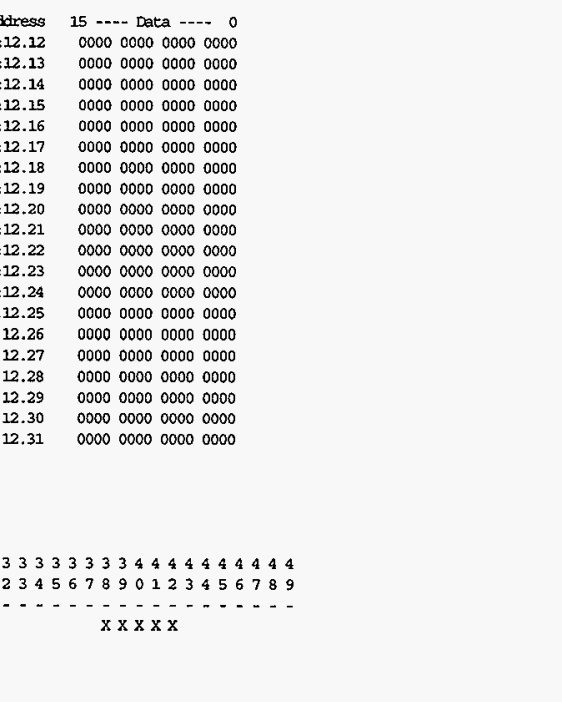

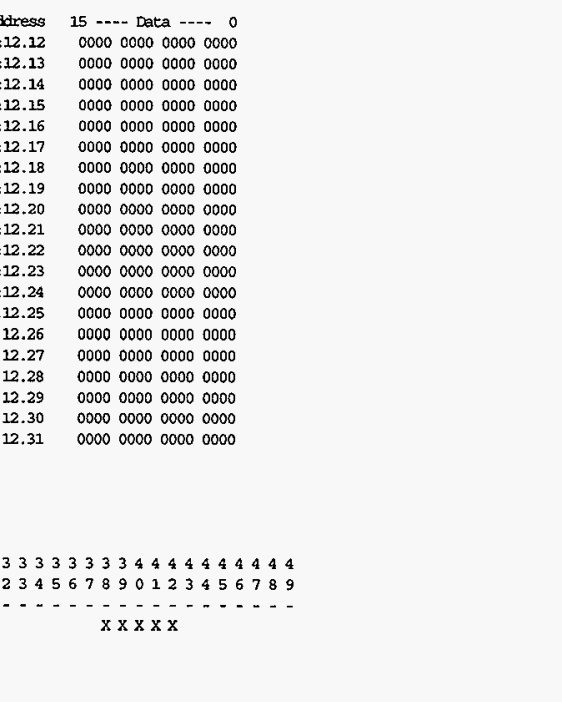

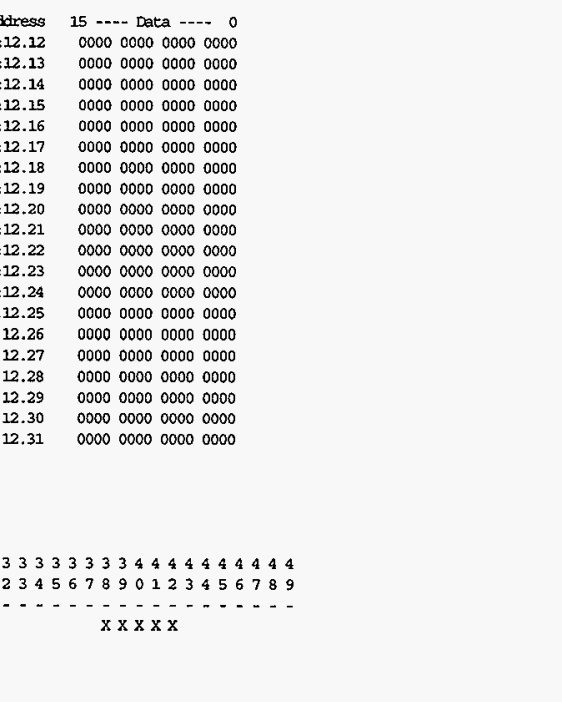

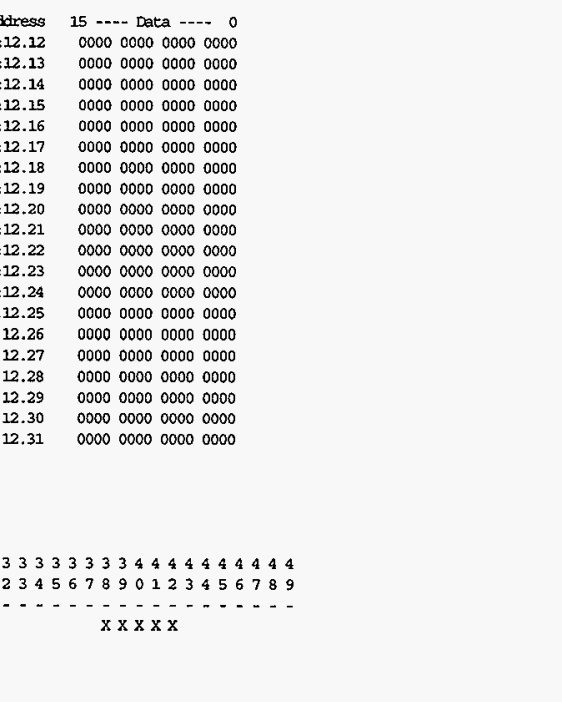

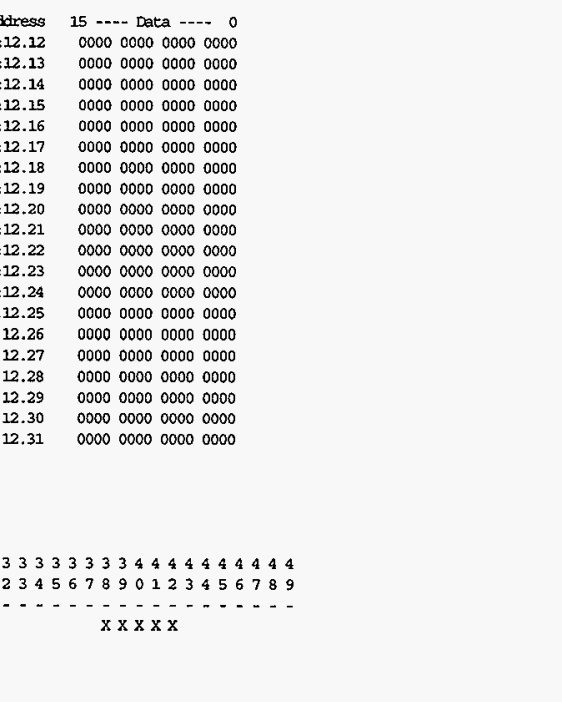

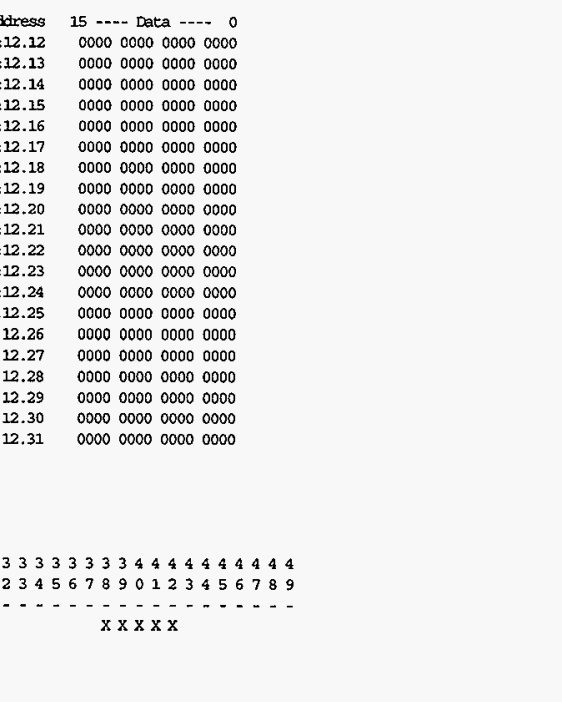

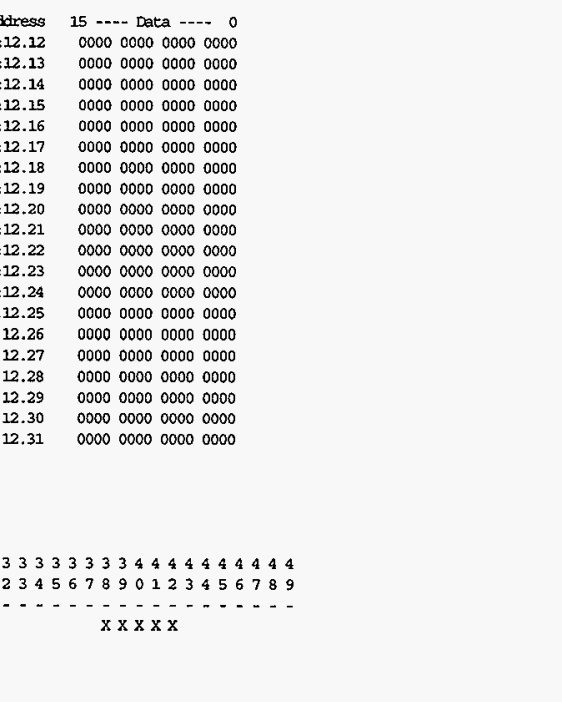

$\begin{array}{ll}15-12 & 0000000000000000 \\ 12.12 & 0000000000000000 \\ 12.13 & 0000000000000000 \\ 12.14 & 0000000000000000 \\ 12.15 & 0000000000000000 \\ 12.16 & 0000000000000000 \\ 12.17 & 0000000000000000 \\ 12.18 & 0000000000000000 \\ 12.19 & 0000000000000000 \\ 12.20 & 0000000000000000 \\ 12.21 & 0000000000000000 \\ 12.22 & 0000000000000000 \\ 12.23 & 0000000000000000 \\ 12.24 & 0000000000000000 \\ 12.25 & 0000000000000000 \\ 12.26 & 0000000000000000 \\ 12.27 & 0000000000000000 \\ 12.28 & 0000000000000000 \\ 12.29 & 0000000000000000 \\ 12.30 & 0000000000000000 \\ 12.31 & \end{array}$
B3/00384 0000000000000000

$\mathrm{B} 3 / 00400 \quad 0000000000000000$

B3/00416 0000000000000000

B3/00432 0000000000000000

$B 3 / 00448 \quad 0000000000000000$

B3/00464 0000000000000000

B3/00480 0000000000000000

B3/00496 $0000000000 \times 00000$

B3/00512 0000000000000000

B3/00528 $000000000000000 \mathrm{X}$

B3/00544 0000000000000000 
Data Eile \#4 Type:T Timer Elements:52 fords:256

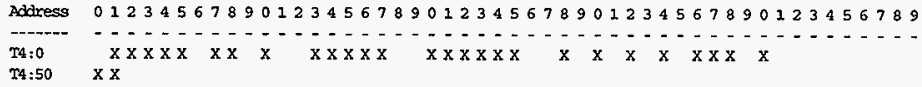

Data File \#5 Type:C Counter Elements:36 hords:108

1111111111222222222233333333334444444444

Adtress

G:0

cis:o

Data File \#6 Type:R Control Elements:38 wonds:114

adiress

1111111111222222222233333333334444444444 $\mathrm{R} 6: 0$ 01234567890123456789012345678901234567890123456789

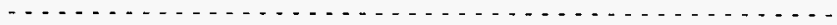
$\times \times \times \times \times \times \times \times \times \times \times \times \times \times \times \times \times \times \times \times \times \times \times$ $\mathrm{x} \quad \mathrm{x} \times \mathrm{X}$

Data File \#7 Type:N Integer Elements:1 Hords:1

1111111111222222222233333333334444444444

Address 01234567890123456789012345678901234567890123456789 NT:0

Data File \#8 Type:F Float Elements:20 hords:40

1111111111222222222233333333334444444444 Address 01234567890123456789012345678901234567890123456789

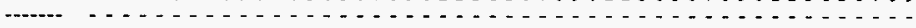
$\mathrm{FB}: 0$ $\mathrm{x} \times \mathrm{x} \times \mathrm{x} \times \mathrm{x} \times \mathrm{x} \times \mathrm{x} \times$

Data File \#9 Type:N Integer Elements:4 hords:4

1111111111222222222233333333334444444444 Acioress 01234567890123456789012345678901234567890123456789 $\mathrm{Ng}: 0$ $\times \times \times \times$

Data File \#10 Type:N Integer Elements: 91 Fords:91

112111 111222222222233333333334444444444 Adoress ----.-. NI0:0 N10:50 01234567890123456789012345678901234567890123456789 - - - - - - - - - - - - - - - - - - - - - - - - - - - - - $\mathrm{x} \times \times \times \times \times \times \times \times \times \times \times \times \times \times \times \times \times \times x$

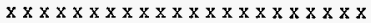
$\mathrm{x} \times \mathrm{x} \times \mathrm{x} \times \mathrm{x} \times \mathrm{x} \times \mathrm{x} \times \mathrm{x \times \times \times \times x}$

Data File \#11 Type:N Integer Elements:37 Fords:37

1111111111222222222233333333334444444444 Adiress 01234567890123456789012345678901234567890123456789 N11:0

Data File \#12 Type:N Integer Elements:3 Hords:3

1111111111222222222233333333334444444444

Adatress 01234567890123456789012345678901234567890123456789 An- . - . - . . . . . . . . . . . . . . . . . . . . . . . . . . . . . . ND2:0 $\mathrm{XXX}$

Data File \#13 Type:N Integer Elements:4 Horcis:4

1111111111222222222233333333334444444444 Adtress 01234567890123456789012345678901234567890123456789 $\log$ -. $\mathrm{x} \times \mathrm{x}$

HNF-2934

Rev 0

A- 129 
Data File \#14 Type:N Integer Elements:43 words:43

1111111111222222222233333333334444444444

Adtress

N $4: 0$ 01234567890123456789012345678901234567890123456789

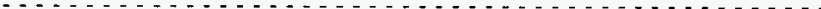

$\begin{array}{lll}\mathrm{x} x & \mathrm{x} & \mathrm{x} \times \mathrm{x} \times \mathrm{x}\end{array}$

Data File \#15 Type:N Integrer Elements:110 Woris:110

1111111111222222222233333333334444444444

Acitress

$-----$

N15:0

N15:50

N15:100

Data File \#16 Type:N Integer Elements:35 Hords:35

1111111111222222222233333333334444444444

Adctress

01234567890123456789012345678901234567890123456789

N16:0

Data File \#17 Type:N Integer Elements:21 hords:21

1111111111222222222233333333334494444444

Addregs

$\mathrm{N} 17: 0$

ax

01234567890123456789012345678901234567890123456789

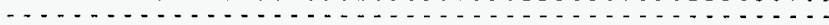

$\mathrm{x} \times \mathrm{x} \times \mathrm{x} \times \mathrm{x}$

Data File \#18 Type:N Integer Elements:36 Hords:36

1111111111222222222233333333334444444444

Address

01234567890123456789012345678901234567890123456789

N.8:0

$$
\mathrm{x} \times \mathrm{x} \times \mathrm{x} \times \mathrm{x} \times \mathrm{x} \times
$$

Data File \#19 Type:N Integer Elements:43 words:43

1111111111222222222233333333334444444444

Address 01234567890123456789012345678901234567890123456789

Mig:0 - 12345678901234567890122345678901234567890123456789

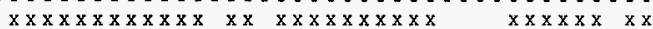

Data File \#20 Type:N Integer Elements:129 Woras:129

1111111111222222222233333333334444444444

Aditress 01234567890123456789012345678901234567890123456789

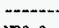

N20:0

N20:100

- - - - - - - - - - - - - - - - - - - - - - - -

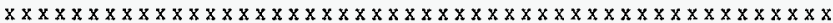

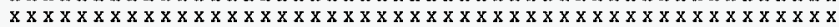
$\mathrm{x} \times \mathrm{x}$

Data File \#21 Type:N Integer Elements:7 words:7

1111111111222222222233333333334444444444

Address 01234567890123456789012345678901234567890123456789

N21:0

Daca File \#22 Type:N Integer Elements:11 Words:11

1111111111222222222233333333334444444444

Address 01234567890123456789012345678901234567890123456789

-.-.-.$\mathrm{N} 22: 0$

Data File \#23 Type:N Integer Elenents:256 Words:256

1111111111222222222233333333334444444444

Adiress 01234567890123456789012345678901234567890123456789

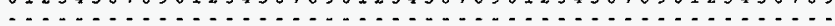

HNF-2934

Rev 0

A- 130 
1111111111222222222233333333334444444444

Address

$--1 \cdot-1$

$103: 50$

N23:100

N23:150

$\mathrm{N} 23: 200$

N23:250

Data File \#24 Type:N Integer Blements:256 Hords:256

Adress

-......

NDA:0

N24:50

N2A:100

N24:150

N24:200

N24:250

Data File $\# 25$

Address

$125: 0$

N25:50

N25:100

N25:150

N25:200

N25:250

Data File \#26 Type:N Integer Elements:256 Hords:256

1121111111222222222233333333334444444444

Address

$--\cdot-$

N26:50

N26:100

N26:150

N26:200

N26:250

Data File \#27 Type:N Integer Elements:256 Words:256

1111111111222222222233333333334444444444

Adtress

N27:0

N27:50

N27:100

N27:150

N27:200

N27:250

Adiress

N28:0 0

N28:50

NE8: 100

N28:150

N28:200

Data File \#29 Type:N Integer Elements:256 Words:256

$\mathrm{x} \times \mathrm{x} \times \mathrm{x} \times \mathrm{x} \times \mathrm{x} \times \mathrm{x} \times \mathrm{x} \times \mathrm{x} \times \mathrm{x} \times \mathrm{x} \times \mathrm{x}$ $\mathrm{xx \times x \times x \times x \times x \times x \times x \times x \times x \times x \times x \times x \times x \times x \times x \times x \times x \times x \times x \times x \times x \times x}$

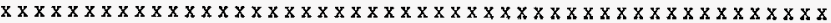
$\mathrm{x} \times \mathrm{x} \times \mathrm{x} \times$

1111111111222222222233333333334444444444 1234567890123456789012345678901234567890123456789 $\mathrm{x} \times \mathrm{x} \times \mathrm{x} \times \mathrm{x} \times \mathrm{x} \times \mathrm{x} \times \mathrm{x} \times \mathrm{x} \times \mathrm{x} \times \mathrm{x} \times \mathrm{x} \times \mathrm{x} \times \mathrm{x} \times \mathrm{x} \times \mathrm{x} \times \mathrm{x} \times \mathrm{x} \times \mathrm{x} \times \mathrm{x}$ $\mathrm{xx \times x \times x \times x \times x \times x \times x \times x \times x \times x \times x \times x \times x \times x \times x \times x \times x \times x \times x \times x \times x}$

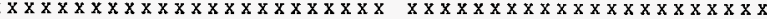

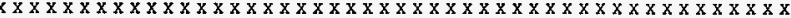

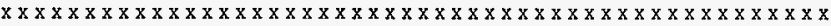
$\times \times \times \times$

1111111111222222222233333333334444444444 01234567890123456789012345678901234567890123456789 $\mathrm{x}$

$\mathrm{x} \times \mathrm{x} \times \mathrm{x} \times \mathrm{x} \times \mathrm{x} \times \mathrm{x} \times \mathrm{x} \times \mathrm{x} \times \mathrm{x} \times \mathrm{x}$

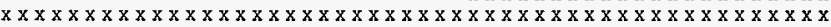

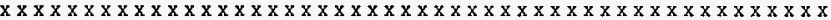
$\mathrm{x} \times \mathrm{x} \times \mathrm{x} \times$

01234567890123456789012345678901234567890123456789

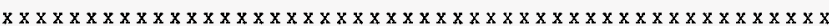

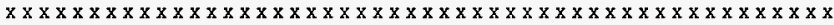

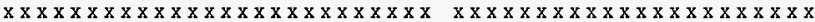

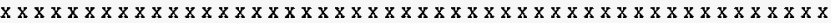
$\mathrm{x} \times \mathrm{x} \times \mathrm{x} \times \mathrm{x} \times \mathrm{x} \times \mathrm{x} \times \mathrm{x} \times \mathrm{x} \times \mathrm{x} \times \mathrm{x} \times \mathrm{x} \times \mathrm{x} \times \mathrm{x} \times \mathrm{x} \times \mathrm{x} \times \mathrm{x} \times \mathrm{x} \times \mathrm{x} \times \mathrm{x}$ $\mathrm{x} \times \times \times \mathrm{x}$ 01234567890123456789012345678901234567890123456789

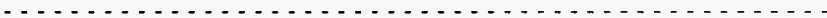
$\mathrm{x}$

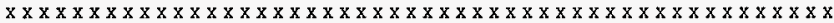

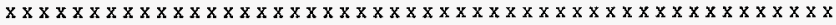
$\mathrm{x} \times \mathrm{x} \times \mathrm{x} \times$

Data File \#28 Type:N Integer Elements:229 Woods:229

1211111111222222222233333333334444444444 01234567890123456789012345678901234567890123456789

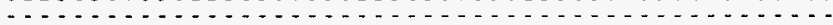

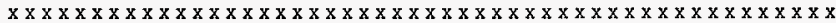
$\mathrm{Xx \times x \times x \times x \times x \times x \times x \times x \times x \times x \times x \times x \times x \times x \times x \times x \times x \times x \times x \times x}$

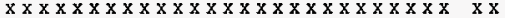

1111111111222222222233333333334444444444 01234567890123456789012345678901234567890123456789 - . . ......................................................

HNF-2934

Rev 0

A-131 
1111111111222222222233333333334444444444

Address

No: 0

$\mathrm{NO}: 50$

N29:100

N29:150

$\mathrm{N} 29: 200$

$\mathrm{N} 29: 250$

Lata File \#30 Type:N Integer Elements:229 words:229

1111111111222222222233333333334444444444

Address

$\mathrm{N3} 0: 0$

N30:50

$\mathrm{NB} 0: 100$

$\mathrm{NBO}: 150$

$\mathrm{NB} 0: 200$

Data File \#31 Type:N Integer Elenents:256 Words:256

1111111111222222222233333333334444444444

Adritess

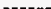

N31:0

N31:50

$\mathrm{N} 31: 100$

$\mathrm{N} 31: 150$

N31:200

$\mathrm{N31}: 250$

Data File \#32 Type:N Integer Elements:229 Wonds:229

1111111111222222222233333333334444444444

Adotress

$\mathrm{N3} 2: 0$

$\mathrm{NB} 2: 50$

$\mathrm{NB} 2: 200$

$\mathrm{NB2}: 150$

$\mathrm{N} 32: 200$

Data File \#33 Type:N Integer Elements:256 Words:256

1111111111222222222233333333334444444444

Axidress

$\mathrm{NB3}: 0$

$\mathrm{N3} 3: 50$

N33:100

$\mathrm{N} 33: 150$

$\mathrm{N} 33: 200$

$\sqrt{3} 3: 250$

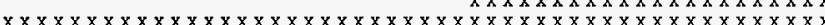

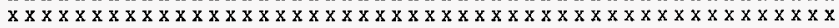
$\mathrm{x} \times \mathrm{x \times x}$

Data File \#34 Type:N Integer Elements:229 horis:229

1111111111222222222233333333334444444444

Address

N34:0

$\mathrm{N} 34: 0$

$\mathrm{N} 34: 100$

$\mathrm{N} 34: 150$

$\mathrm{NB} 4: 200$

Data File \#35 Type:N Integer Elements:140 hords:140

1111111211222222222233333333334444444444

Adatress

N35:0

$\mathrm{x} \times \mathrm{x} \times \mathrm{x} \times \mathrm{x} \times \mathrm{x} \times \mathrm{x} \times \mathrm{x} \times \mathrm{x} \times \mathrm{x} \times \mathrm{x}$

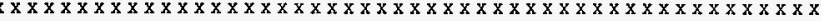

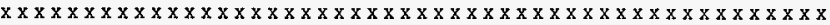
$\times \times \times \times \times \times$

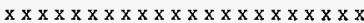

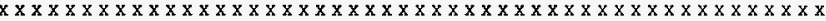
$\mathrm{x} \times \mathrm{x} \times \mathrm{x} \times \mathrm{x} \times \mathrm{x} \times \mathrm{x} \times \mathrm{x} \times \mathrm{x} \times \mathrm{x} \times \mathrm{x} \times \mathrm{x} \times \mathrm{x} \times \mathrm{x} \times \mathrm{x} \times \mathrm{x} \times \mathrm{x} \times \mathrm{x}$ $\mathrm{x} \times \times \times \times \times$ 01234567890123456789012345678901234567890123456789

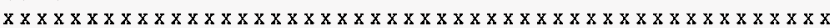

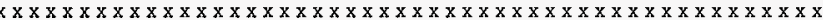
$\mathrm{x} \times \times \times \times \times \times \times \times \times \times \times \times \times \times \times \times \times \times \times \times \times \times \times \times \times \times \quad \times \times$

01234567890123456789012345678901234567890123456789 $\mathbf{x}$ 01234567890123456789012345678901234567890123456789

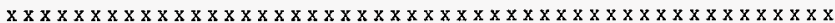

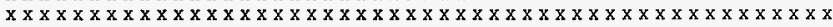

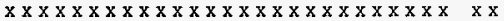
01234567890123456789012345678901234567890123456789

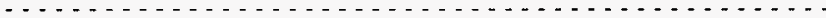

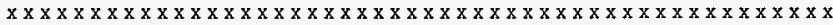

HNF-2934

Rev 0

A-132 
1111111111222222222233333333334444444444

Address 01234567890123456789012345678901234567890123456789

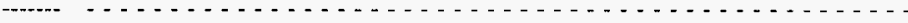

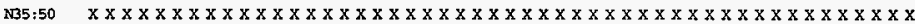

N35:100 $\times \times \times \times \times \times \times \times \times x$

Data File \#36 Type:N Integer Blements:93 Words:93

1111111111222222222233333333334444444444

Address 01234567890123456789012345678901234567890123456789

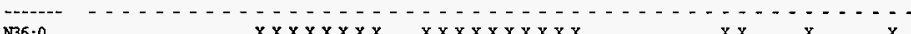

$\times \times \times \times \times \times \times \times \quad \times \times \times \times \times \times \times \times x$

$\mathrm{x} x$

Data File \#40 Type:N Integer Elements:43 wonds:43

1121111111222222222233333333334444444444 Adiress 01234567890123456789012345678901234567890123456789

NO:O 
Slot Fart \# Card Description

\begin{tabular}{|c|c|c|}
\hline 0 & $2747-1532$ & 5/03 CFU - 32K Merrory - cs301 \\
\hline 1 & 1746-NI4 & 4 Channel Analog Irqut Mocule \\
\hline 2 & 1746-NI4 & 4 Chanrel Analog Irqut Nocule \\
\hline 3 & 1746-NIO4I & 2/2 Channel Analog I/O Mochlle \\
\hline 4 & $1746-\mathrm{NI} 4$ & 4 Channel Analog Iryout Module \\
\hline 5 & $1746-\mathrm{NI} 4$ & 4 Garmel Analog Trout Mochle \\
\hline 6 & 1746-NIOAV & 2/2 Chamel Analog I/O Mocule \\
\hline 7 & $1746-1012$ & 6-Irpout 100/120 VAC, 6-autput (RLY) \\
\hline 8 & 1746-1012 & 6-Imput 100/120 VAC, 6-Dutput (RLX) \\
\hline 9 & 1746-1012 & 6-Irquit 100/120 VAC, 6-autput (RLX) \\
\hline 10 & $1746-1012$ & 6-Irquit 100/120 VAC, 6-Output (RLY) \\
\hline 11 & $1746-\mathrm{NT} 4$ & 4 \%. Thertiocoaple Iruput Module \\
\hline 12 & $1747-5 \mathrm{~N}$ & RIO \$carner \\
\hline
\end{tabular}

HNF-2934

Rev 0 
Quantity Part \# Cand Description

Quantity Part

4 1746-IO22 6-Irqut 100/120 VAC, 6-Output (RIY)

4 1746-2II4 4 Chamel Analog Input Module

1 1746-NIO4I 2/2 Channel Aralog I/O Module

1 1746-NIOAV 2/2 chamel Analog I/O Module

1 1747-SN RIO Scanner

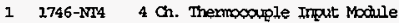


Fortable 1000 CFM Exhanster Program SLC-500 I/O Parts Location Report

I/O Foint I/O (Symbol) Description

Device Descriotion

Upper DES Lower DES

HNF-2934

Rev 0

A-136 